\title{
CRITICAL PHENOMENA
}

\author{
IN \\ SUPERCONDUCTORS
}

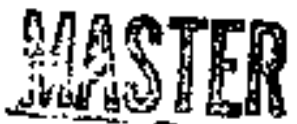

\author{
James Clark Solinsky
}

\section{Solid State and Low Temperature Physics Group}

SCHOOL OF PHYSICS AND ASTRONOMY

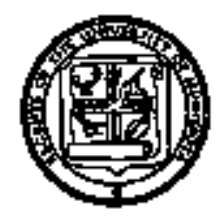

DECEMBER 1973

UNIVERSITY OF MINNESOTA

MINNEAPOLIS, MINNESOTA

\section{Work supported in part by the U.S. Atomic Energy Commission}




\section{DISCLAIMER}

This report was prepared as an account of work sponsored by an agency of the United States Government. Neither the United States Government nor any agency Thereot, nor any of their employees, makes any warranty, express or implied, or assumes any legal liability or responsibility for the accuracy, completeness, or usefulness of any information, apparatus, product, or process disclosed, or represents that its use would not infringe privately owned rights. Reference herein to any specific commercial product, process, or service by trade name, trademark, manufacturer, or otherwise does not necessarily constitute or imply its endorsement, recommendation, or favoring by the United States Government or any agency thereof. The views and opinions of authors expressed herein do not necessarlly state or reflect those of the United States Government or any agency thereof. 


\section{DISCLAIMER}

Portions of this document may be illegible in electronic image products. Images are produced from the best available original document. 


\title{
CRITICAL PHENOMERA
}

IN

SUPERCONDUCTORS

\author{
A THESIS \\ SUBNITTEO TO THE FACULTY OF THE GRAOUATE SCHOOL \\ OF THE UNIYERSITY OF MINHESOTA
}

BY

JAMES CLARK SOLINSKY

IN PARTIAL FULFILLMENT OF THE REQUIREMENTS

FOR THE OEGREE OF

DOCTOR OF PHILOSOPHY

\section{DECEMBER 1973}

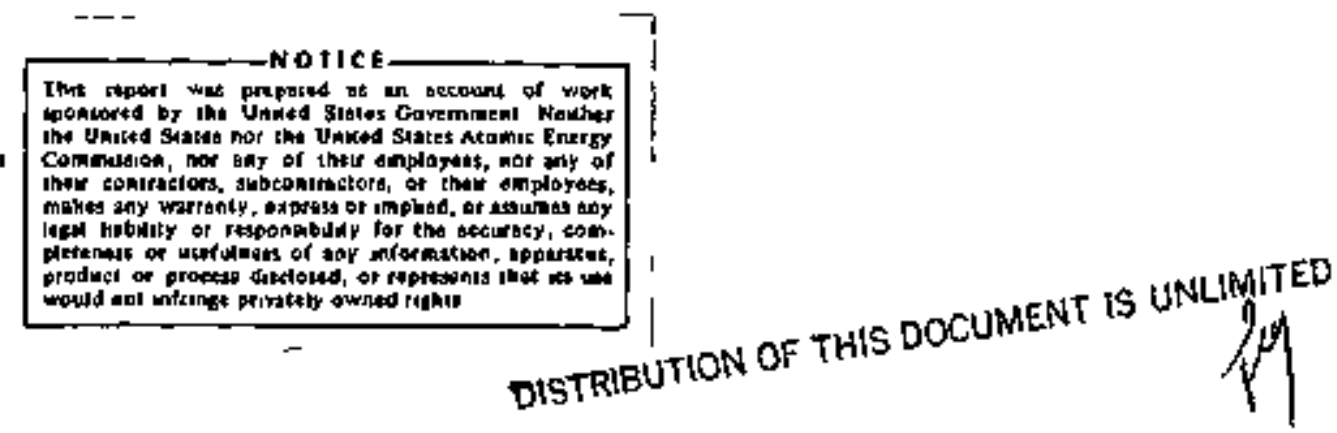


Abstract

Critfcel Phenoriona in Superconductors

Departures from the predfctions of Mean Fleld Theory (MFT) are not usually observed because the region in temperature $\Delta T_{C}$ (1.e., the critical region) over which the theory is invalid is very small $\left(\sim 10^{-12} \mathrm{~K}\right.$ ) for pure superconductors. The narrowness of this region results from the dependence of $\Delta T_{c}$ on the inverse of the sixth power of the zero temperature value of the temperature dependent coherence length $E(T)$, a parameter whtch measures the size of coherent superconducting regions, and is about $10^{4} \&$ for pure superconductors. This relationship between $\Delta T_{c}$ and $\xi(0)$ is why in superfluid $\mathrm{He}^{4}$ critical phenomena are observed $(\xi(0) \sim 2 \AA)$ and why MFT works so well for pure superconductors. But $\Delta T_{C}$ can be increased to the order of $10^{-3} \mathrm{~K}$ by studying dirty superconductors $(1, e$, the mean free path $i \ll(0))$ in restricted geonatry (1.e., a characteristlc length $L \ll \xi(0))$.

Exparimental studies were carried out with 1000 \& thick dirty granular aluminum fi1ms (mass $\sim 20 \mu \mathrm{g}$ ) which were evaporated onto mice substrates. The electrical conductivity and heat cepacity ware studied in the superconducting transition region. The resistive transition of the f1im was used as a themiometer in confunction with opticel heating in an A.C. calorimetry schene to masure the filius $5 \times 10^{-9} \mathrm{~J} / \mathrm{K}$ heat capac1ty. Results presented on 
four films indicate a rise in the heat capacity near the low temperature region of the transition where the film's resistance becomes zero. This rise peaked in two fins studied to a value of up to 32 times the BCS jump in heat capacity and at a temperature of about $20 \mathrm{mK}$ below the MFT transition temperature $T_{c}^{M F T}$ as determined from the conductivity measurements. An applied magnetic field widened and reduced the peak in the hest capacity while shifting it to a lower temperature. These results indicate that higher order corrections are needed to recent heat capacity predictions for thin $f 11 \mathrm{~ms}$ in the transition region.

oj 
Preface

Because dissertations tend to be written and then placed on obscure sheives, this thesis was structoeed to enable the reader to read wat is important.without all the sinall details. The main body of the thesis is without all the experimental details, and is written from a phenomenological point of view, with fine theoretical detalls to be found in the references. All experimental dotalis are structured in appendices, and can be omittod In reading. If one so chooses. If this format does not make the thesis more readable, it at least nelped the author in distingutshting botween what is called research and wat is called physics. 
Table of Contents

page

Abstract............................................

Preface, ............................................... 1it

Table of Contents...................................... iv

List of Tables and Flgures................................ if

Chapter 1. Introduction, $\ldots \ldots \ldots \ldots \ldots \ldots \ldots \ldots \ldots \ldots \ldots \ldots$,

Chapter 2. Theory................................... 9

Fluctuation Enhancemant...................... 9

Estimation of the Critical Region................. 13

Theoretical Predictions.......................... 15

Chapter 3. Previous Experinental Work...................., 29

Chapter 4. Experiment................................ 42

Basic Methods.................................. 42

Apparatus $\ldots \ldots \ldots \ldots \ldots \ldots \ldots \ldots \ldots \ldots \ldots \ldots \ldots, 45$

Equivalent Circutt.......................... 46

Error Analysts ............................ 56

Chapter 5, Data Analys is and Discussion...................65

Generat features............................... 65

Electrieal Conductivity......................... 69

Heat Copacity............................... 74

Magnetic Ffeld Effects......................... 77

Discussion.................................., 83

Conctuston.................................. 87

List of Roferences $\ldots \ldots \ldots \ldots \ldots \ldots \ldots \ldots \ldots \ldots \ldots \ldots \ldots \ldots \ldots$ 
Appendix A, A.C. Bridges and Thermometry..................... 95

Appendtx 8. Optical Systens................................. 104

Append1 x C. Cryostat and Isolation..........................., 110

Appendix 0 , squids................................... 118

Appendix E, Functional Offferentiation and Humerical Analysis...... 133

Appendfx F, A,C. Calorimetry and the Heat Conduction Problem....... 146

Appendix G. Analog Servo System......................... 166

Appendix H. Sample Preparation and Resistive Transitions......... 177

List of References for Appendices $\ldots . \ldots \ldots \ldots \ldots \ldots \ldots \ldots \ldots, \ldots \ldots, 202$

Acknowladganents . ....................................... 207 


\section{List of Tables and Figures}

page

Ftgure 1. Lahibda Transition in LHe ${ }^{4} \ldots \ldots \ldots \ldots \ldots \ldots \ldots \ldots \ldots$.

Figure 2, List of Phase Transitions $\ldots \ldots \ldots \ldots \ldots \ldots \ldots \ldots \ldots, 2$

Table 1. Cottical Regions for Offferent Systems............. 7

F1gure 3. Predicted Fluctuation Heat Capacity Effects in

Three Dimensions.............................. 12

Figure 4. Hartree Approximetion Calculation for Three

Dimensions..................................... 12

Table 2A. Heat Capacity Predictions for Superconductors......... 19

Table 2B. Conductivity Predictions for Suporconductors......... 21

F1gure 5. Predicted Fluctuation Heat Capacity in One Dimension... 25

Figure 6. Observed Fluctuation Heat Capacity in Two Dimens fons... 25

Figure 7. Finite Anomaly Prediction in Two O1mensions.......... 26

Figure 8. Finite Anomaly Prediction in Two Dimansions compared to Hartree Result................................ 26

Figure 9. Observed Heat capactity of Pure Superconductors in

Three olmensions.............................. 30

Figure 10. Magnetic Field Heat Cepact ty Studies in Otrty,

Bulk Superconductors.......................... 30

Figure 11. Comparison of Magnetic Field Studies to Hartree One Dimensfonal Predictions.......................... 33

Figure 12, Calorfmeter Used by Za1ly and Mochel.............. 33

Figure 13. Top Vien of Calomimeter Used by Zally and Mocher...... 35

Figure 14, Rew Calorimetry Dat on BI FIlms.................., 35 
Figure 15. Excess Heat Capacity and Resistive Transition of Bi FtIm.

Figure 16. Comparison to Hartree Prediction for Bf Fflm Results.. "37

Figure 17. Resfstive Transition Used as a Non-linear Thermometer. 43

Figure 18. Equivelent Circuit Used in Analysis............... 43

Figure 19. BTock Diagram of Electronics Used in Experinent....... 48

Ftgure 20. Raw Data used in Experiment..................... 61

Figure 21. Heat Capacyty of $1000 \AA$ Aluminum Ftim $(\$ 388) \ldots \ldots \ldots 63$

Table 3. Physical constants for Filus ofscussed.............. 66

Figure 22. Resistive Transition From Run $\$ 39$ with

Theoretical fit................................ 71

Figure 23. Inverse of Excess Conductivity Ratio vs. Temperature

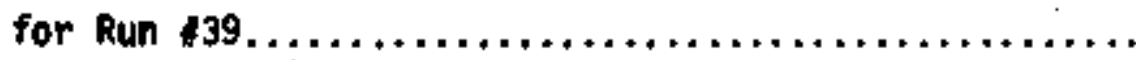

F1gure 24. Total Heat Capecity of Run \$30I, Plotted for Three

Temperature Ranges........................... 76

Figure 25. Resist1ve Transftion for Run 368 , Showing Multiple

Transttions............................... 78

Figure 26, Resistive Transitton and Heat Capacty for Rum $\$ 368 . . .79$

Figure 27. Excess Heat Capacity Plotted for Run $339 . \ldots \ldots \ldots \ldots, 81$

Figuro 28. Inverse of Excess Heat Capactity for Run $\$ 39, \ldots \ldots \ldots, 82$

F1gure 29. Total Heat Capacity for Rum $\$ 39$ for Two Values of Magnetic Field.................................. 84

Figure A-1. A.C. Resistance Bridge.......................... 96

figure A-2, Self-Batencing Bridge........................ 100

Figure A-3. A.C. Kelvin Bridge........................ 101

Figure B-1. Optical Electrontcs........................ 105 
Figure B-2, Output of Minfature Light Bulbs................ 105

Figure C-1, Dewar Assembly and Isolation Platform,............ III

Figure C-2. LHe Leve] Detector Circuit.................... 114

Figure C-3. Cryostat for Experfment...................... 115

Flgure 0-1. Basic Equations for squid 0peration.............. 119

Figure $0-2$, squio Construction and Biasing.................. 122

F1gure D-3. SQUID Electronics--Mixer and Osctllator........... 125

Figure D-4. SQUIo Electroni cs..Pre-Antp................... 126

Fi gure 0-5. SqUID D.C. Bias and Feedback Box.............. 128

Figure 0-6. Souid Critical Current Behavior................ 132

Figure E-1. Basic Analog Differentiation................. 135

Figure E-2. More Complicsted Analog Oifferentiation........... 137

Figure E-3. Higher Order Harmonic Detection Method............. 139

F1gure E-4. Small Modulation Different1ation................. 140

F1gure E-5. Multiplier Box............................ 141

Figure F-1. A. C. Calorimetry and Electrical Analog.,......... 147

Table F-1. ETectrical Anslogy to Heat Flow.................. 149

Figure F-2. A.C. Heat Conduction for a \$teel Rod.............. 155

Tablo F-2. Thermal Properties for Materials Used in the

Experiment. ............................... I59

Figure 6-1. Linear and Mon-Linear Relationships for Servo System. 169

Figure G-2. Block Dlagram of Analog Computer................ 171

F1gure G-3. Non-Linear Functions Produced by Analog Computer..... 172

Figure G.4. Active Filter Box........................ 174

Figure 6-5. Block Diagram of Complete Servo System............ 775

Figure H-1. TEM Micrographs of ATuminum Films................ 179 
FIgure H-2. Sample Geonetrles and Seif-Supported Method......... 183

Figure H-3, Resistive Iransition of a Self-Supported F11m....... 189

Figure H-4. Time Dependence of "Notch" in Rasistive Transition,.. 190

F1 gure H-5. Magnatfc Broadening of Resistive Transitions......... 193

Figurn H-6, Cumulative Data Compared to Patton's Theory......... 198 
Chapter 1. Introduction

Among the many processes that one observes in the physical world, one of the most striking processes is that of a phase transition. A phase transition is characterized by a dramatic overall change in system, brought about by a relatively small change in one of the parameters of the system. Figure 1 shows the heat capacity of 11 quid $\mathrm{He}^{4}$ plotted vs. temperature. One should note the dramatic change in the heat capacity due to a retatively smatl change in the temperature. One should also note that as one increases the resolution of the variable parameter (temperature), the relative shape of the feature remains the same. This is a characterfstic of the "critical region," and is related to the exponentfal power of the diverging quantity (see ref. 2; scaling theory). Ont can observe critical behavior in other systems as well. A more common exelopio is the lrausd-gas phase transition. One can ponder for a long time the question of why water bolls at precisely one temperature and becomes a wholly different state of matter from the liquid.

Landau ${ }^{3}$ first realized the similarity in all phase transitions. He belleved a phase transition to bedue to a change in symmetry of the systen which he characterlzed wf th a paraneter called the "order parameter." For temperatures greater than the transf tion tomperature $\left(T_{c}\right)$ the system was in a dfsordered state, and the order parameter ( $\Delta$ ) had zero average value. For temperatures 


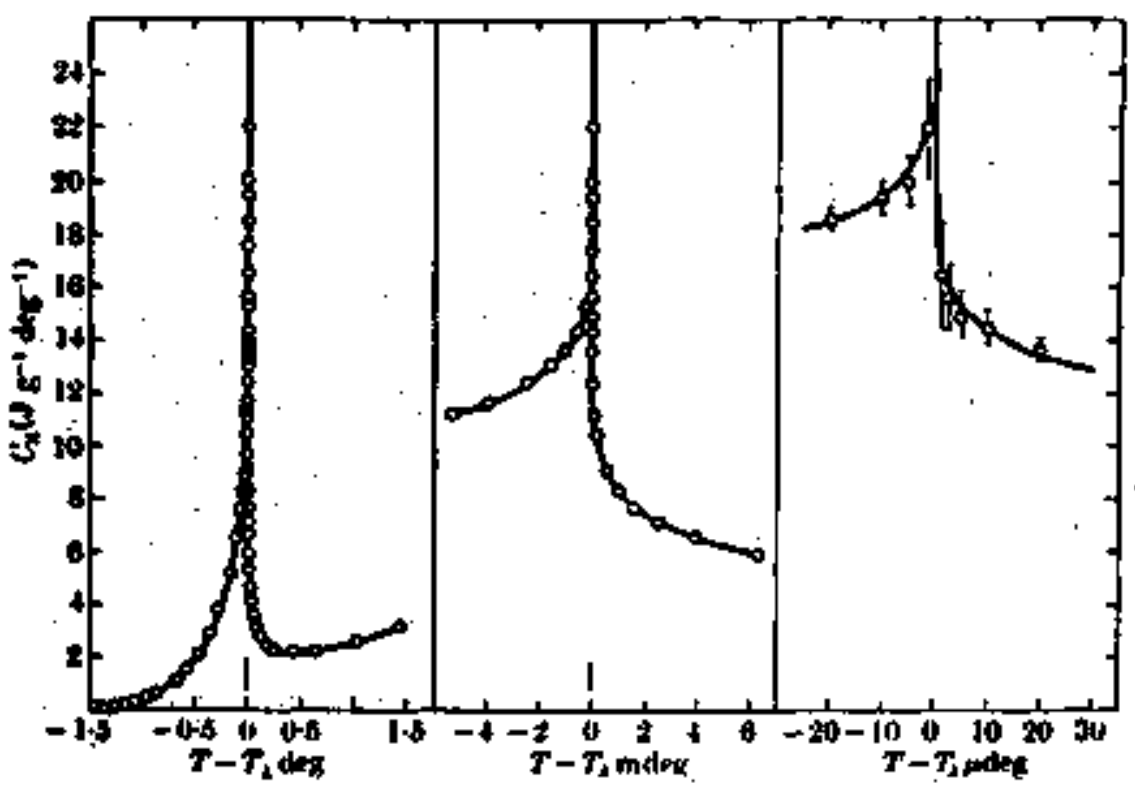

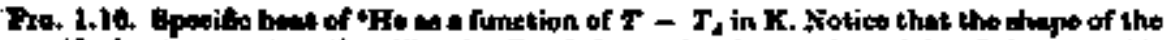

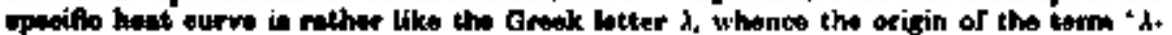

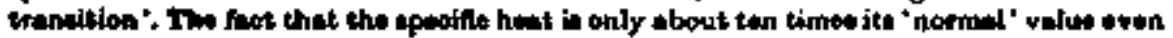

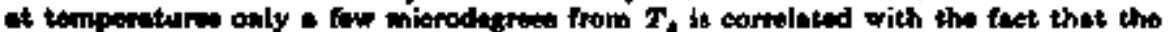

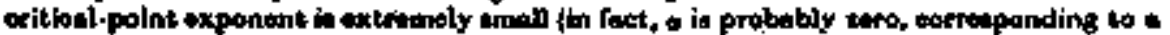
logatrithmie divergates. The width of the emall vertical jine just above the origin indi.

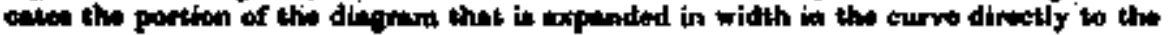

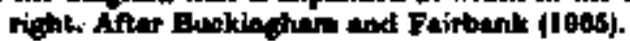

Figure I. (fron Ref, 1)

Partial list of phase transitions and the order posnmeters $\langle p\rangle$ essocintal twith each. Adapled from Kadanoff of al. (J007)

\begin{tabular}{|c|c|c|c|}
\hline Tronstition & 3iraning of (p) & Free chotee in 'pl & $\begin{array}{l}\text { Therimolyineinis } \\
\text { conjugente of }\langle p\rangle\rangle\end{array}$ \\
\hline liquid-7es & $\bullet-\infty$ & 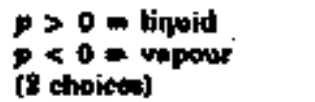 & $\mu$ \\
\hline ontifirromentipntie & embetices & 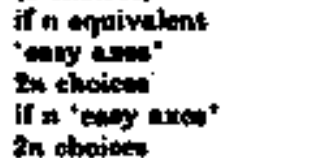 & 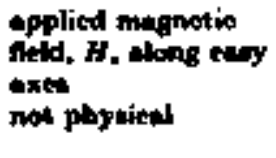 \\
\hline $\begin{array}{l}\text { Heimintert modid } \\
\text { ferremernet }\end{array}$ & mongmiantion $\boldsymbol{M}$ & $\begin{array}{l}\text { direction of } \Delta I \\
\text { tcas ehoow any velue } \\
\text { on swrince of epherel }\end{array}$ & $\boldsymbol{H}$ \\
\hline $\begin{array}{l}\text { Ioling modkel ferro. } \\
\text { magnet. }\end{array}$ & * & 2 ehoices & $H$ \\
\hline wepropoducton & $\begin{array}{l}\Delta \text { (comples popp } \\
\text { perometer) }\end{array}$ & Fhang of A & not plyymical \\
\hline erpertidid & (i) (rotwlenchle wave & phate of $\langle\phi\rangle$ & not pliy연) \\
\hline $\begin{array}{l}\text { terrocisetrio } \\
\text { phen separtition }\end{array}$ & $\begin{array}{l}\text { Letive poleriantion } \\
\text { concentrulion }\end{array}$ & $\begin{array}{l}\text { finite number of } \\
\text { choikes } \\
2 \text { chaike }\end{array}$ & $\begin{array}{l}\text { electrio field } \\
\text { edifithese uf } \\
\text { clremital potentials }\end{array}$ \\
\hline
\end{tabular}

Figure 2. (fron Rof. 1) 
less than the transition temperature, the system was in an ordered state, and the order paraneter had a non-zero average value. This is summarized below:

Landau Phase Trans1tions

$\begin{array}{lll}T>T_{c} & |\Delta|=0 & \text { disordered } \\ T<T_{C} & |\Delta| \neq 0 & \text { ordered }\end{array}$

An example of the ordar paramater can be shown by looking at a Curfe-Weiss spin lattice, where $N_{+}$is the number of spins up, and $N_{-}$is the nuwber of spins down. Then $\Delta=\left(N_{+}-N_{-}\right) /\left(N_{+}+N_{-}\right)$ and then the order parameter obays the rules shown above. The universality of the order paraneter ldea is shown in Figure 2. The transition is listed in the first colum with the order parameter listed in the second. The third colum is the degeneracy, or "free cholce" in the order parameter. One can see that for the superconducting transition, the order parameter is the complex energy gap parameter ( $\Delta\}$, sometimes written as $\Psi \quad\left(=\mathrm{Ae}^{i \phi}\right)$, wich is $\mathrm{like}$ a quantum mechanical condensate wave function with its amplitude (A) relating to the square root of the density of electron pairs (Cooper pairs) in the system, and has a phase ( $\phi)$ associated with it. One can add to the table the recent observations in Llie ${ }^{3}$ at $2.7 \mathrm{mK}$ of a possible phase transition similar to the pairing of a superconductor.

Landau used a phenomenological approach, which for a 
superconductor has an equivalence in the microscopic B.C.S. ${ }^{4}$ theory near $T_{c^{*}}$. Ho expanded the thermodynamic free energy function as a power series in the order parameter with temperature depondent coefficients, where certain terms dropped out due to symmetry orguments. Thus, the fros energy is:

$$
F=F_{n}+\Delta|\Delta|^{2}+\frac{B}{2}|\Delta|^{4}+\cdots
$$

where $F_{n}$ is the free energy above the transition temperature. This is a type of Hean field Theory (MFT) and is consistent with second ordar phase transftion in a superconductor (the name originally reluted to the ordar of the discentinuous derivative of the free energy, but now just implies no latent heat in the phase transition). Th1s can be shown by minimizing the froe energy with respect to the order paraneter and calculating the heat capacitfes above and below $T_{c}$ (where $c=T \frac{\partial^{2} F}{\partial T^{2}}$ )

At finite temperstures, above the critical temperature, it is possible to have thermelly activated fluctuations in the order parameter. Then, the thermal average value of the order paramater is zero ( $\langle\Delta\rangle=0$ ), but the r:m.s, fluctuation in the order paranter about the therial average value at a point $r$ is not zero $\left\langle\langle\delta \Delta\rangle^{2}=\left\langle(\Delta(r))^{2}-\langle\Delta(r)\rangle^{2}\right\rangle\right)$. Thus, to modify the MFT, a spatialiy varying term is added to the free energy $\left(C|\nabla \Delta|^{2}\right.$ ) and eq. (1) becomes:

$$
F=F_{n}+\Delta|\Delta|+\frac{B}{2}|\Delta|^{4}+C|\nabla \Delta|^{2}+\cdots
$$


In the MFI with fluctuations, conmonly celled fluctuation theory. The corretation function between two fluctuating regions can be calculated as;

$$
g\left(\vec{r}, \vec{r}^{\prime}\right)=\left\langle(\Delta(\vec{r})-\langle\Delta(\vec{r})\rangle)\left(\Delta\left(\overrightarrow{r^{\prime}}\right)-\left\langle\Delta\left(\overrightarrow{r^{\prime}}\right)\right\rangle\right)\right\rangle
$$

as was done by Kadanaff. ${ }^{2}$ One finds a coherence length $(\xi(T)\}$ or range of this correlation function and a relaxation time $(1 / \Gamma(T))$ associated with thase flactuations above the critical temperature, where:

$$
\begin{aligned}
& \xi(T)=\xi(0) \varepsilon^{-t / 2} T>T_{c} \text { where } \epsilon=\frac{T-T_{c}}{T_{c}} \\
& \Gamma(T)=r(0) \quad E^{1}
\end{aligned}
$$

The relaxation time comes from the more complicated Time Dependent Ginzburg-Landau equation (T.D.G.L.). ${ }^{5}$ One can picture regions on the stze of $[\xi(T)]^{3}$ in the system going superconducting for a time on the seale of $J / \Gamma(T)$ at temperatures above the critical temperature, in the so-called "classical region".

As one approaches closer to the critical temperature, the difference in entropy between the two states approaches zero (1.e., the definition of and order trenstion is $\left.\Delta S\right|_{T c}=0$ ). Then, it takes very 11ttle change in entropy for the order parameter to fluctuate between the two states, and the fluctuations get more 
violent. Since, the probability for the order parameter ( $A$ ) to be betwoen $\Delta$ and $(\Delta+\delta \Delta)$ varies as the exponential of the entropy, for $T\rangle T_{C}$ where $\langle\Delta\rangle=0$, this is the same as the probabllity for the order paraneter being equal to the fluctuations $\langle\delta \Delta\rangle$. For small differences in the entropy between the two states, this probab1lity is a Gusstan function of the difference in entropy. Thus, when one approaches the transition, the entropy difference approaches zero, and the probabtlity of the flucturations being an the order of the order parameter $(\langle\Delta\rangle,\langle\Delta\rangle$ ) becomes enormous. One can see that the size of the fluctuations diverges, and their lifotime becomas infinite (eqs. (4), (5)), so that the system is approaching coherent macroscopic mode. In this region, the MFT theory breaks down because of the diverging quantities, and one calis it the "critical region". The width of this region can be ost fmated in reduced temperature $(\varepsilon)$ as shown below.

$$
\varepsilon_{c}=\frac{T_{-T}}{T_{c}} \sim\left[\frac{k_{B}}{\Delta c s_{0}^{3}}\right]^{2} \quad(\text { ref. 2) }
$$

This estimate is similar to the thermal energy in the size of the coherent region $\left(k_{B} T_{C} / \xi_{0}^{3}\right)$ being on the order of the average energy associated with the phase transition ( $\left.T_{C} \Delta C\right)$, and where the exponent comes from the temporature dependence of $\xi(T)$. Table 1 ilsts a number of systems with their zero tempersture coherence Soe Landay and Lifshitz, statistical Phys1cs, (1969), pg. 343. 
7

Table I

a

System

Magnetic

Ferroelectric

Lh He 4

Superconductor

$$
\begin{gathered}
\xi(T-0) \\
\leq 2 \AA \\
100 \AA \\
1 \% \\
104 \AA
\end{gathered}
$$

M.F.J.

Ho Good $\left(\epsilon_{\mathrm{c}}<10^{-2}\right)$

Good $\left(\varepsilon_{c}<10^{-4}\right)$

No Good $\left(\varepsilon_{\mathrm{c}}<10^{-1}\right)$

$\operatorname{cood}\left(E_{\mathrm{c}}<10^{-12}\right)$ 
length used in estimating the critical region. Thus, it is obvious why there are observable critical phenomena in LHe 4 , and why for $*$ superconductor one would expect M.F.T. to work welt and no observable critical behavior to exist.

The estination of the critical region is somewhat arbitrary. and wach calculation requires apecific estimation of its breakdown. But equation (6) is calculated from more general considerations. and is in major agreement with the estimates of others. One can then imagine the understanding of the phase transition in a superconductor as consisting of phenomanological M.F.T. regions (where the theory works weil) on either side of the crftical region and are a sood approximation for intcroscopic B.C.S. theory. One uses approximations to approach closer to the critical region, to include fluctuations. And finally, in the critical region the M.F.T. with fluctuations breaks down and one can only use scaling theory. B.C.S. theory (including Gor'kov's integral equations) being a wicroscopic description, will work well throughout, but the calculations can be impossible near the critical region. Oae can estimate that in a superconductor, the critical region, and thus the region of divergent behavtor, is extremely narrow, and one would expect to never observe such behavior. Essentially the description of a superconductor in the critical region is as difficult to predfet theoretically as it is to observe experimentally. 
Chapter 2. Theory

\section{Fluctuation Enhancement}

Because of the rather narrow regions in temperature predicted for abservable erftical behavior, one could conclude that in superconductors one would never observe fluctuation effects. There are, however, means of increasing the fluctuations of a superconductor to enhance the fluctuation effects. This enhancement will not only widen the estimated critical region, but will also change the nature of the fluctuation behavior, and in addition, lower the transition temperature.

Anderson ${ }^{6}$ made on analogy with other phase transitions and noted that "dirty" superconductors, where the electron mean free path was smalier than the coherence length $(2<<\quad \xi(0)$ where now $\left.\xi(0)=.85 \sqrt{l} \xi_{0}\right)$, should have an enhanced critical region. The electrons cannot travel as fast, due to the more numerous collisions, and are not as effective in averaging out the fluctuations that lead to critical and fluctuation effects.

Little's ${ }^{7}$ work in microgeonstries led to the suggestion that reduced dimensions would enhance fluctuations. Reducing the dinenstions of a sample simply inlifies one of the characteristic lengths of the system $(L)$ is less than the coherence length $(\xi(0))$. Thus, a 3-D system is bulk mater1at; $2-D$ is a thin film (thickness - I) ; 1-p is a wire (area $=L^{2}$ ); and O.D is a powder (particle vol ume $=L^{3}$ ). One can imaglne a bill bouncing in a 3-0 box, and, as one oushes in two opposing sides, the bouncing increases as the system becomes a plane. It is this type of effect, in a quantum 
mechantcal sense, that allows the reduced goometry to enhance fluctuations.

Hore recently a third method has been suggested by Lee and Shenoy ${ }^{8}$ and others, to increase fluctuations. If one applies a small magnetic field to metal, the motion of the electrons in the metal wilt be confined to planes (2-0) and (for higher fields) to 1-D rods. Thus, an applied magnetic field will reduce the dimensionality of the systera to further enhance the fiuctustions in a superconductor.

One can also think of fluctuations as an "inexact" pairing of the electrons in forming Cooper pairs. ${ }^{6}$ for the B.C.S. formulation, one has a total momentum equal to zero, but fluctuations allow local, non-prectsion pafring. This is equivalent to the Hartree-Fock self-consistency treatment of the phenomenological approach where one has a total constant value of the order parameter over space, but the $|\nabla \psi|^{2}$ term fimlies a local non-constant value. At temperatures above the transition $\left(T>\mathrm{T}_{\mathrm{C}}\right)$, fTuctuations are observabie because there is no pair-field $(\langle\psi\rangle=0)$, and one observes "shorting" effect in the conductivity measurements due to small fluctuating regions going superconducting. But, for $T<T_{c}$ the problean is quite different. Now a pair-field exists $(<\psi\rangle \neq 0)$ and fluctuations are only slightly disturbing the whote field. Then, regtons going nomal due to fluctuations in the pair fiold are hard to observe amidst the whole superconducting field. It is for this reason that the treatment of fluctuation 
theory is generally concerned with fluctuations above $T_{c}$, and Why the theories, as well as the experimental data, are not as complete below $T_{c}$.

Historically, work began in looking at the conductivity of dirty superconducting fijms. The wide transitfons observed were at first believed to be due to sample inhomogenteties, where one was observing an averaging of many $T_{c}$ 's in the transition. But, at the same time, theoretical work began that predicted observable fluctuation effects outside the critical region, even in pure superconductors. These calculatians agreed with the widened experimantally observed transitions. Fluctuation effects have been observed in the conductivity $(0)$, in the diamagnetis: $\left(X_{d i a}\right)$, and in the superconducting tunneling $\left(I_{c}\right)$ of a superconductor above Its MFT transition temperature. Approximation schemes used in the calculations lead to good agreement between fluctuation theory and experimental results near the transition. The overall offect of enhanced fluctuations is to widen the predicted critical region (as.large as $\varepsilon_{c} \sim 10^{-3}$ for diety, 2-D films) and change the nature of the measurement and shift the transition temperature to a lower value.

Figure 3 shows the fluctuation effects on the heat capacity of superconductor as calculated by Grossman, Richter, and Wissel]. ${ }^{9}$ Hotice not only the 1nereased distortion of perfect B.C.S. discontinuous Jump in the heat capacity, as one widens the critical region, but also notfce how the effect is appearing at a 


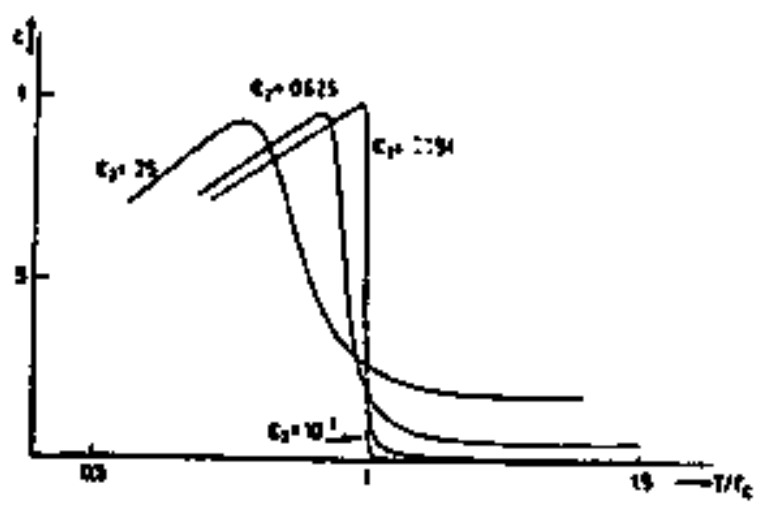

FIG. 2. Specific heat of filse to various transithor widths $\epsilon_{\mathrm{y}}$ -

Figure 3. (fran Ref. 9)

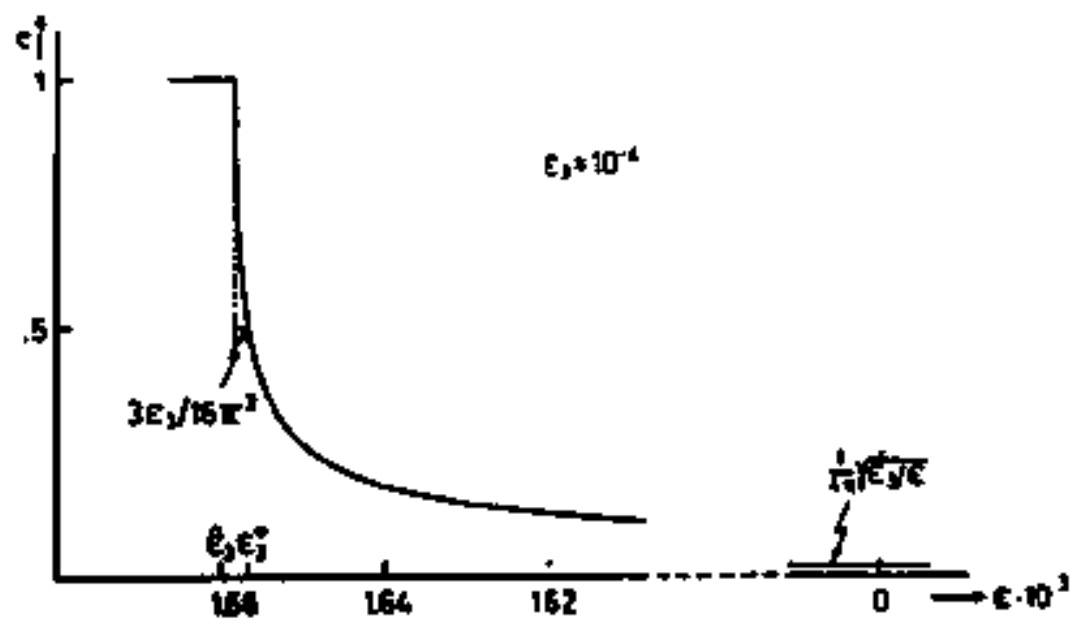

FTO. 1. Bulk epecific heat in Heatree mpproximation.

Figure 4. (from Ref, 9) 
lower transition temperature for wider eritical regions $\left(\varepsilon_{\mathrm{c}}\right)$. Also notice the "rounding" ar precursor nature of the effect as one approaches the transition from above. This is similar to the form that the other measurements $\left(\sigma, x_{d i a}, I_{c}\right)$ of fluctuations show (1.e., precursl ve entrance into divergant behavior).

As stated earlier, much work has been done in looking at fluctuation effects in the superconducting transition, through. $0, X_{d i a}$, and $I_{c}$. But, because of the experimental difficulties, not much work has been done to look at the heat capacity fluctuation effects. Before entarking on the theoretical nature of these effects, one should revise the estimates of the critical reglon with enhanced fluctuations to see if the experiment is possible.

\section{Estimation of the Critical Region}

As stated earlier, the estimation of the critical region is a somawhat arbitrary procedure. It basically involves estimating where a particular calculation breaks down as $\varepsilon\left(=\frac{T-T_{C}}{T_{C}}\right)$ gets smaller. Thus, if one made an approximation of neglecting the 4th order term in the fres energy $\left(|\Psi|^{4}\right)$, then the critical region could be defined as when the omission of the 4th order term no longer becomes possible $\left(|\Psi| \mathcal{L}|\Psi|^{2}\right)$ relative to the lower order terms. Depending upon the particular calculation, most estimates of the crittcal region can vary by an order of manitude or so. Farrell's ${ }^{6}$ estimates for the above mentioned 
breakdown are 11sted below for the dirty superconductor case only. The estimate is for alumf num, chosen because of its ease in film preparation and moderate critical temperature, and because of the extensive previous work reported in the literature on airty aluainum.

Dimension

Quantfty

Rumerical Value for dirty Al uminum $(2 \times 10 \AA)$

3-D

$$
\varepsilon_{c} \sim \frac{1}{\left(k_{F} \varepsilon_{0}\right)} \frac{1}{\left(k_{F}\right)^{3}}
$$

$10^{-8}$ bulk

2mD

$$
E_{c} \frac{1}{\left(k_{F} L\right)} \frac{1}{\left(k_{F} \ell\right)}
$$

$10^{-5}(1000 \&$ thick $=L)$

1-D

$$
\varepsilon_{c} \frac{1}{\left(k_{F} L\right)^{4 / 3}}\left(\xi_{0} / 2\right)^{1 / 3}
$$
$10^{-3}(1000 \AA$ A di aneter $=L)$

Here $k_{F}$ is the Feral momentum $\left(\sim 1.75 \times 10^{8} \mathrm{~cm}^{-1}\right), \xi_{0}$ is the Pippard intrinsic coherence length $\left(\sim T .6 \times 10^{-4} \mathrm{~cm}\right)$, and $\mathrm{L}$, were defined earlier. For a $\mathrm{ft} 1 \mathrm{~m}$, one can use the fact that $\sigma=\frac{\mathrm{Ne}^{2} \ell_{\text {eff }}}{\mathrm{Niv}_{\mathrm{F}}}$ to get $E_{c}=0.43 \times 10^{-5} \mathrm{R}_{\square}^{\mathrm{N}}$ for aluminum filns (see ref. 10) where $R^{N}$ is the normat resistance of the $f i l n$ per square. These estimates show that only in the film, and wire experiments would one expect to observe critical behavior. But, it should be pointed out that this does not rule out observable fluctuation behavior for regions wuch wider than $\varepsilon_{c},\left(\varepsilon>\varepsilon_{c}\right)$ as long as the inhomogenefties are small ( $\varepsilon_{\text {inhom. }}<\varepsilon$ ). One woutd then expect it to be possible to see a fluctuation enhanced transition in a dirty alunimum film. 


\section{Theoretical Predictions}

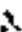

To study critical phenomena in superconductors, an experiment was done in dirty aluminum fflms. The characteristics of the fllms which were studfed, were the electrical conductivity and heat capacity. The conductivity was studied only as a guide to the transition properties of the films and to relate results to other work in the $i$ terature. The real interest was in the heat capacity in the vicinity of the transition. The heat capactity is a static thermodynamic property related to the energy density-energy density corretation function $\left(\left\langle\delta \psi^{2}\right)\left(\delta \psi^{2}\right)>\right.$ since Fa $\psi^{2}$ and where now will be used for $\left.\Delta\right) .^{6}$ The conductivity is a dynamical transport property related to gradients in the ordar parameter correlation function $\left(\left\langle\left(\psi \nabla \Psi^{\prime}\right)\left(\Psi^{\prime} \nabla \psi\right)\right\rangle\right\}^{6}$ one would not necessarily expect these. two properties to have the same tenperature dependence in the transition region. If one considers the fact that the aluminum f13ms ore quite granular, and intergrain tunneling is Included in the conductivfty as an effective electron mean free path, one would even expect less simtilarity. Also, as pointed out earifer, the conductivity is a measurement of a property of the order parameter that is not symmetric about the transition, whereas the heat capacity has some symietry to its behavlor. It is anly when one considers that large fluctuations are present, and that the behavior of these properties is governed predominantily 
by the size of the coherence in the system, that one could then expect the behavior for both propertfes to be related in the seme way to the temperature dependent coherence length (E(T)).

The theoretical approsch to calculating these properties In the transition region breaks down into essentially three methods. One can take the rather crude 61nzburg-Landau free energy presented earlier ( $F$, and form the spatial average of the order paraneter by computing functional integral of the order paraneter weighted by the Boltzmann factor. Thus, the free energy becones in fourter transformed momentum spece:

$$
F(\psi)=\sum_{r}^{i \vec{q} \cdot \vec{r}} F(\psi(\vec{r}))
$$

and then $\left\langle\{\psi\}^{2}\right\rangle$, the thermal average of the order parameter Is:

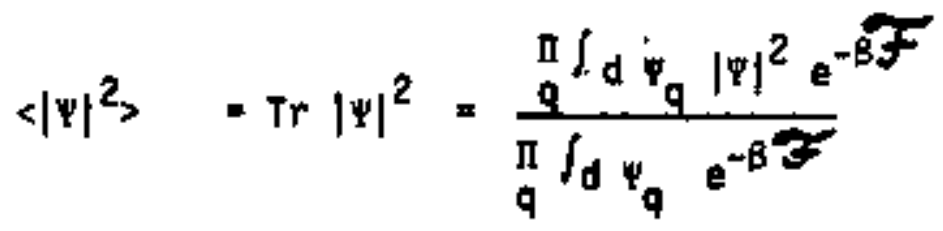

This is an integral equation similar to a Green's function propagator and usually involves a momentum cutoff in the integration. Because of the non-linear nature of the G-L free energy, one has to make approximations to $F$ in order to solve for $\left\langle|\Psi|^{2}\right\rangle$. Then one can calculate properties of the system by using transport 
or thermodynamic relationships of the order parameter to the properties calculated, which is $51 \mathrm{mi}$ ]ar to operating on the free energy explicitly. The problem with this method is that the G-L equation Is phenomenological, and the absolute values of the coefficients are not known, so in the end one has to match to experiment by varying these parameters. It 8150 is an inexact calculation in that one cannot solve the problem exactly, because of the diagramatic perturbation technlques used in the integral equation.

The second approach to the problem is to 90 to the microscople B.C.S. Hawlitontan and calculate the pair-field propagator In thermal Gren's function analysis, and use diagranmetic techniques. Near $T_{c}$, as pointed out earlier, this is equivalent to the G-L approach, except it has a wider range of validity, as long as no approximation methods are used. Then the coefficients are known, and one usually has only one parameter in the theory $\left(T_{c}\right)$. But, this problem too has to rely on perturbation methods, and one agatn is using approximation methods to handle the nonIinear free energy. This mothod is in essence, the real understanding on a microscopic level, but it does not have the machinery to complete the predictions exactly.

The third method of approach is that of $\mathrm{Ma}^{11}$ and Wilson using r. - normalization technfques where they are concerned with an n-parameter field in a d-dimensional space. The problem is formulated in aneral manner (expansion in powers of $1 / n$ ), and then 
one looks at a set of diagrams and concludes which are the important sets in certain 1 imits of $n$, and d (for a superconductor $n=2$ ). There is no perturbation theory used th the general formulation because one sums the diagrams, rather than explicitly evaluating them, so it has the possibility of solving the problem exactly. But it is mainty concerned with the regitons of the transition where all quantities are related only to the size of the coherence length. It also cannot be solved at this point in time, without some use of perturbation theory to get around the mechanical difficulties (an example is ref, 15).

The calculation of properties of a superconductor in the transition region thus involves approximation schemes. This leads to the rather Targe range of predictions of the behavior of the heat capectity and the conductivity in 3,2 , and I dimensions both below and above $\tau_{c}$. Some author's calculations will leave out certain terms. Others will Inel ude them and show different behavior. But, net ther have included all the terms, so it is difficult to show that - higher ordor term might not cancel off some effects of an earlier calculation. For this reason, the calculated predictions of interest are 11sted in Table $2 \mathrm{~A}$ for the heat capacity and listed sor the conducitivity in Table $2 B$ for all three values of $d$ (dirnension). The different approximations are shown, and some idea of the validity is given.

Above the transition, there is wide spread agreement with the conductivity calculations in all three dimensions. The 2-D result 
Isto 2h. Wat Cupectly (C).Predittions for 01rty sugerconductors (un lest

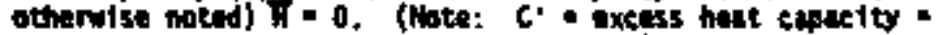

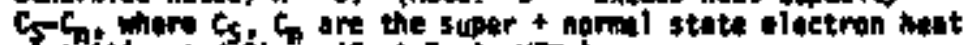

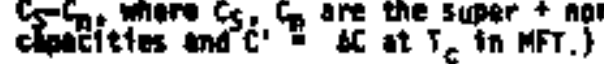

\begin{tabular}{|c|c|c|c|c|}
\hline (disistaton) & Aproxinat ion & Authors (ret. D) & 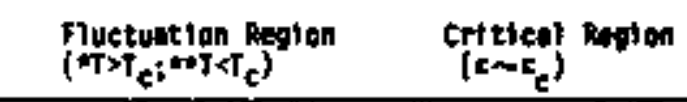 & Co:ments \\
\hline 3-pure & 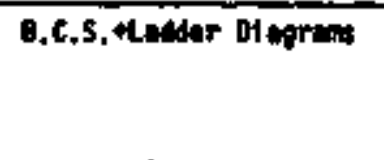 & 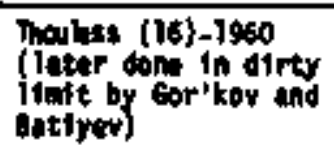 & 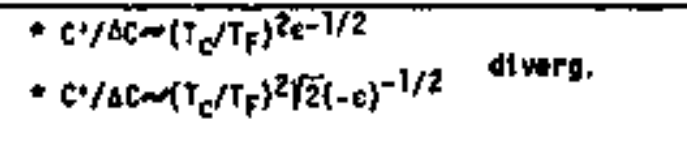 & $\begin{array}{l}\text { Sun diverses } \\
\text { for tot }\end{array}$ \\
\hline $\begin{array}{l}3 \text { aeneral } \\
\text { and order }\end{array}$ & $\begin{array}{l}6-t+\left.|\sigma|\right|^{2} \text { in } \\
\text { Functionsl integret }\end{array}$ & Leveruut $(17)-1964$ & $\| c+\Delta C$ a $\varepsilon^{-1 / 2}$ & $\begin{array}{l}\text { Calculstion for } \\
\text { 2nd order con- } \\
\text { anred to quertz }\end{array}$ \\
\hline 3-pure & $\begin{array}{l}\text { 9.M. Stetistical } \\
\text { Grginents using } \\
\text { Grien's fusctions }\end{array}$ & Wuar (18) -1955 & no singulerity for supureonductor & \\
\hline 3 & $\begin{array}{l}\text { microscopic c B.C.S. } \\
+ \text { Green's functions }\end{array}$ & $\begin{array}{l}\text { ssiansop lontin } \\
(24) \cdot 1968\end{array}$ & $\begin{array}{l}C^{*} / \Delta C \text { a } c^{1 / 2} \\
C^{\prime} / \Delta C \text { a } e^{-2}\end{array}$ & $\begin{array}{l}\text { c, o have same } \\
\text { fluctuation } \\
\text { regions }\end{array}$ \\
\hline$z$ & 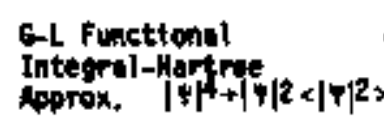 & 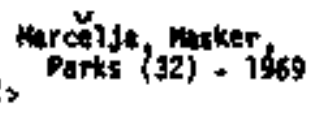 & $\because c$ lac of fint te anomaly below $T_{c}^{M r T}$ & \\
\hline 3 & 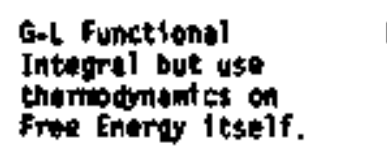 & Ferrell $(6) \cdot 1 \% 9$ & $\begin{array}{l}c / \Delta t a c c^{-1 / 2} \\
C 1 / \Delta t \times e^{-1}\end{array}$ & \\
\hline $\begin{array}{l}3 \\
2 \\
3\end{array}$ & $\begin{array}{l}\text { Gol Functional Integrsi } \\
\text { lst order onty }\end{array}$ & 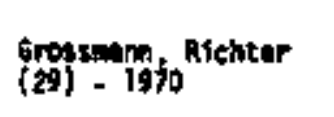 & $\begin{array}{l}\cdot c^{\prime} / \Delta \cos e^{d / 2-2} \\
* c^{\prime} / \Delta \cos (-e)^{d / 2-2}(\sqrt{2})^{d-2}\end{array}$ & \\
\hline
\end{tabular}


Tobie 2A (continued)

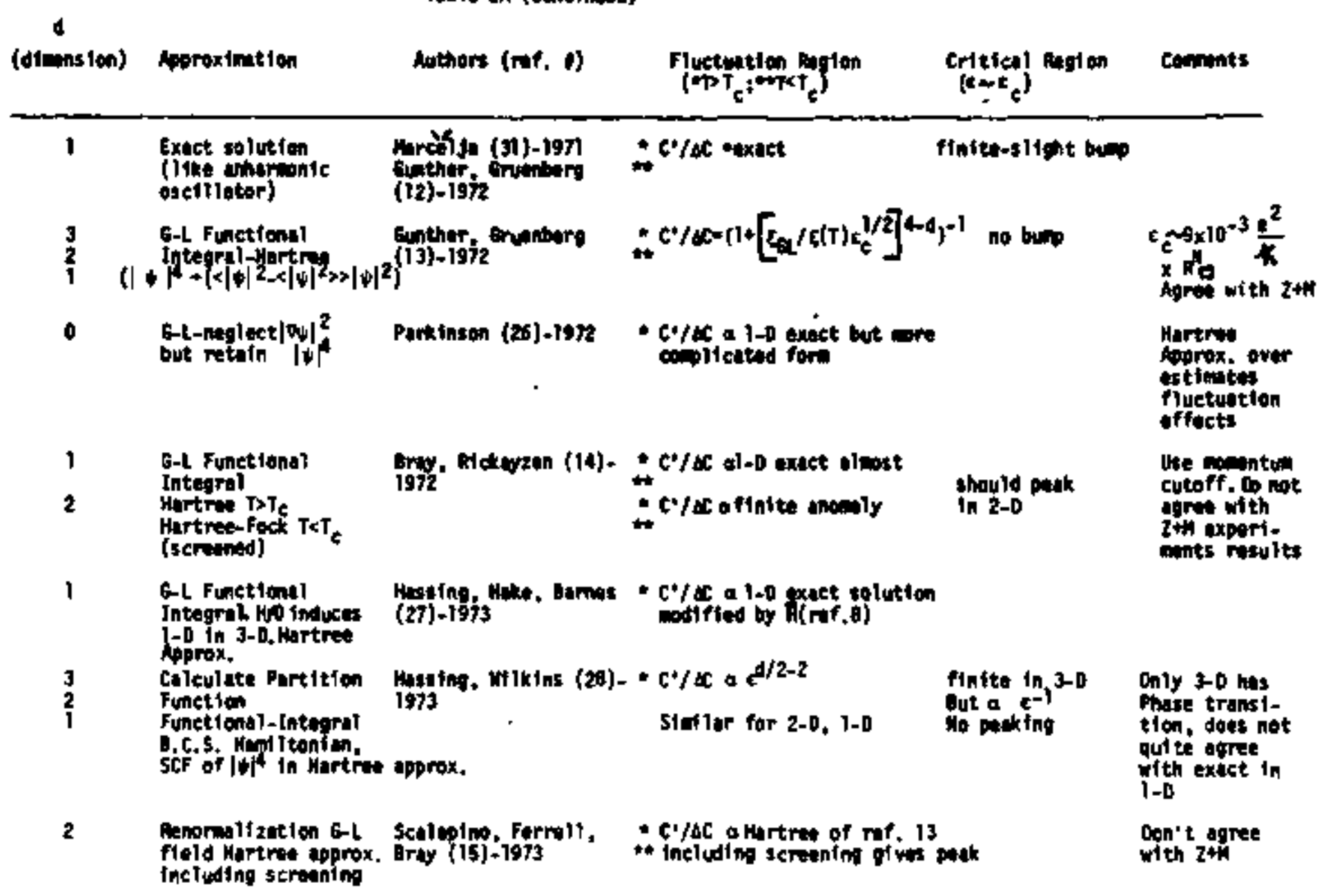


Table 2s. Conductivity (o) Oredictions for of nty superconduetors (

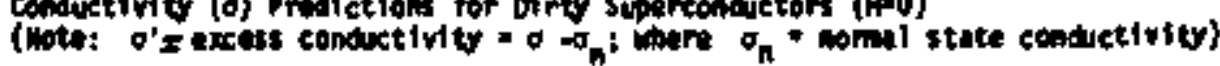

\begin{tabular}{|c|c|c|c|c|}
\hline (atimenten) & Approxtutlen & Muthors (ref. I) & 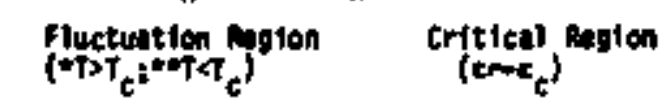 & Coimments \\
\hline 方 & $\begin{array}{l}\text { tes + Events } \\
\text { functiens }\end{array}$ & 4lengy, Larkin $(23,24)$ & $\cdot \nabla 1 / \sigma_{n} a c^{d / 2-2}$ & \\
\hline$\frac{3}{2}$ & $\begin{array}{l}\text { scs + frotion's } \\
\text { functionit }\end{array}$ & Het $(25)-1968$ & 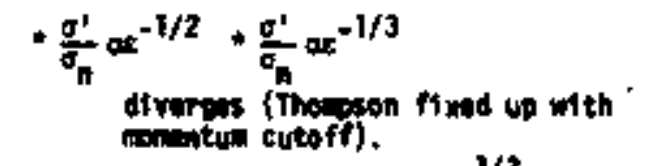 & $\begin{array}{l}\text { Use scr for } \\
\text { spectron in } \\
\text { criticel nolom }\end{array}$ \\
\hline $\begin{array}{l}3 \\
2 \\
1\end{array}$ & 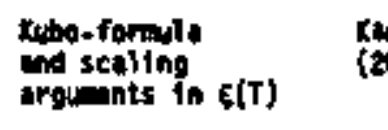 & 20)-isos & $* \sigma^{1} / a_{n} a e^{d / 2-2} \sigma^{1 / a_{n}}$ a $\left(\frac{\varepsilon}{\varepsilon_{c}}\right)^{-1 / 4}$ for $3-0$ & $\begin{array}{l}\text { Many assunptions } \\
\text { in arguntents }\end{array}$ \\
\hline 2 & & & 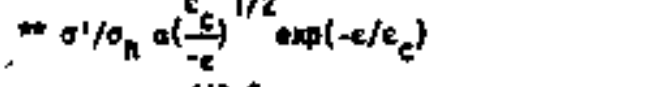 & \\
\hline $\begin{array}{l}3 \\
2 \\
1\end{array}$ & 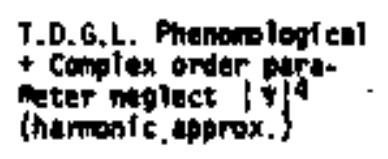 & $\begin{array}{l}\text { Sctuldt (21) }-1968 \\
\text { Sctioldt }(22)-1970\end{array}$ & $\begin{array}{l}* \sigma^{1} / \sigma_{n} a e^{d / 2-2} k(0) \\
* \sigma^{1} / o_{n} a / d^{d / 2-2} k(0)\end{array}$ & 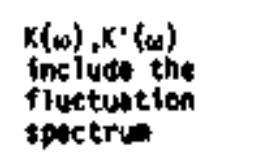 \\
\hline 2 & 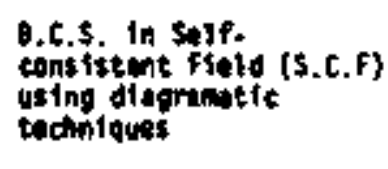 & Tt unukt (19)-1969 & 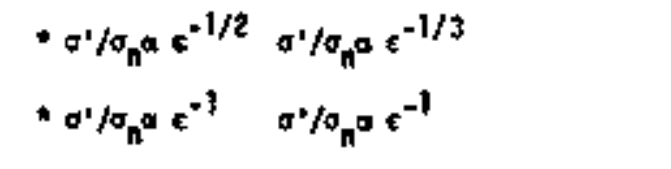 & \\
\hline$\frac{3}{2}$ & $\begin{array}{l}\text { G-L Functional Integral } \\
\text { worts on fret anergy }\end{array}$ & Forrinll (6)-1869 & $\begin{array}{l}+\sigma^{1} / \sigma_{n}+e^{-1 / 2} \\
+\sigma^{1} / 0_{n}+e^{-1}\end{array}$ & $\begin{array}{l}\text { Expects o, } \\
\text { to havin save } \\
\text { tomperature } \\
\text { dapendence }\end{array}$ \\
\hline
\end{tabular}


Table at antimed

\begin{tabular}{|c|c|c|c|c|}
\hline (tolingion) & Aproxication & Augnors (ret. o) & flucturation Rertion & tomants \\
\hline 2 & 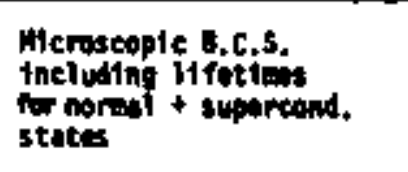 & $\begin{array}{l}\text { Pattan (30) - 1971 } \\
\text { (full expartiontu1 } \\
\text { compurisen in nf. 33) }\end{array}$ & 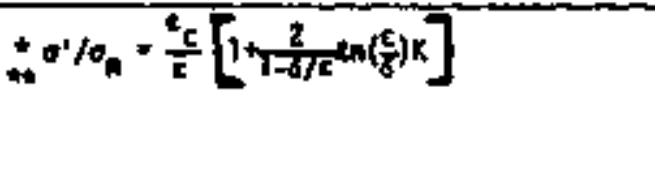 & $\begin{array}{l}\text { No transtition } \\
\text { in } 2-0,1.0\end{array}$ \\
\hline $\begin{array}{l}3 \\
2 \\
1\end{array}$ & 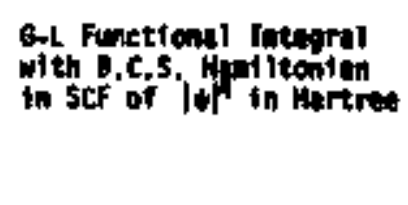 & 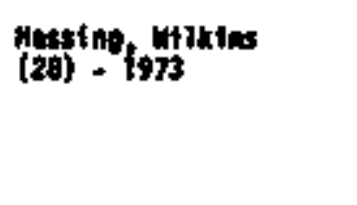 & 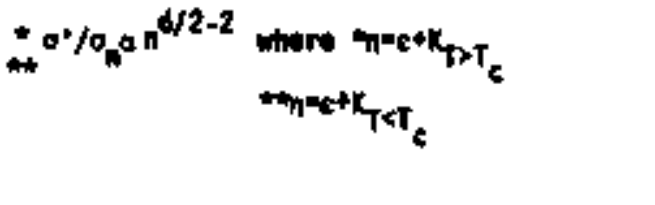 & 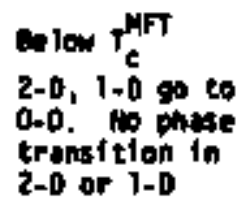 \\
\hline 3 & $\begin{array}{l}\text { G-L Functionnl Inteogral } \\
\text { Hartren hpprox. }\end{array}$ & $\begin{array}{l}\text { matker mareallia, } \\
\text { Parks }(52)-1969\end{array}$ & & \\
\hline 2 & & & $=0$ & \\
\hline 」 & & & $=\sigma^{*} / \sigma_{n}$ a $(-\varepsilon)^{3}$ & \\
\hline
\end{tabular}


(generally associated with the work of As lamazov and Larkin) for the excess conductivity is:

$$
\sigma \% \sigma_{n}=\tau_{0}^{A . L .} E^{-1} \tau_{0}^{A . L .}=1.52 \times 10^{-5} R_{D}^{N} T>T_{c}
$$

The Makt and Thompson corrections are for films with long eTectron mean free paths, and thus include the electron-pair interactions. Patton's work is in agreement with the largest amount of experimental work, but it still is valid only outside the critfcal region. Betow $T_{c}$, there are varfous forms of exponential falling off that can be fit to experiments only for $T<T_{C}$. There is really no clear understanding of the conductivity very near $T_{c}$, or below, and generaly there is accepted agreement in the A.L: result above $T_{c}$. There is some agreement that 2-0 and I-D systems do not exhibit a phase transition, but fust an inexact superconductivity, with a very large (but not infinite) conductivity. There is also some agreanent that the conductivity and the heat capacity should have the same temperature dependence. Table $2 \beta$ is by no means a complete 1 ist, but only an illustrative table of the meny ways theorfsts can get simitiar or different answers. It would be expected that dfrty atuminum films would at least follow the A.L. result above $T_{C}$.

The etleulations of Table $2 \mathrm{~A}$ show some of the earlfer work predicting a divergence in lower order perturbation theory. It mabe is an artfact of a theory that there is a divergence. That is, 
if an approximation is used in the calculation, it breaks down close to $T_{c}$, and the calculation diverges. This does not mean that the full calculation would necessartiy diverge. Thus, in some ways the precurstye nature of the heat capacity prediction is a property of the approximation. Later work Includes better approximation methods for higher order terms. These results predict different types of fintte anomalies in 2-D. The 1-0 problem can be solved exactly, and is used as check on the approxtmation methods in 2-0. Figure 4 shows the precursive nature of a 3-0 calculation, with possibly a finite anomaly at the reduced $T_{c}\left(\varepsilon_{j}=\varepsilon_{c}\right)$. Figure 5 shows the exact $1-D$ calculation, with a slight bump in smooth transition over to the B.C.S. Jump. Figure 6 shows one prediction of the 2-D result compared to an experiment (Zally and Mochel referred to as $\mathrm{ZM}$ ) to be discussed Tater. Figure 7 shows a finite anomaly prediction in $2-0$ that is in disagreemant with the work of $Z M$, where $A$ is a momentum cutoff. In general, all the calculations predict above the transition:

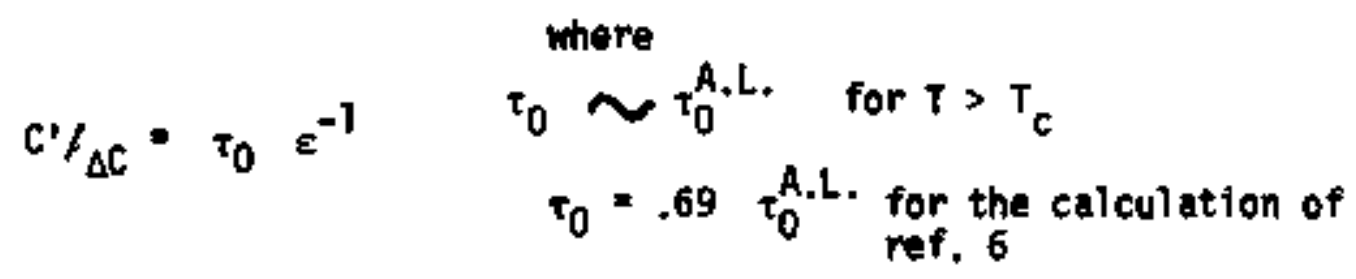

This exhibits a precursive nature similar to the conductivity. But, the difference in the predictions is for the critical region. wher some theorles predict a peaking of the heat capacity above the B.C.S. Jump, and others predict monotonic transition 


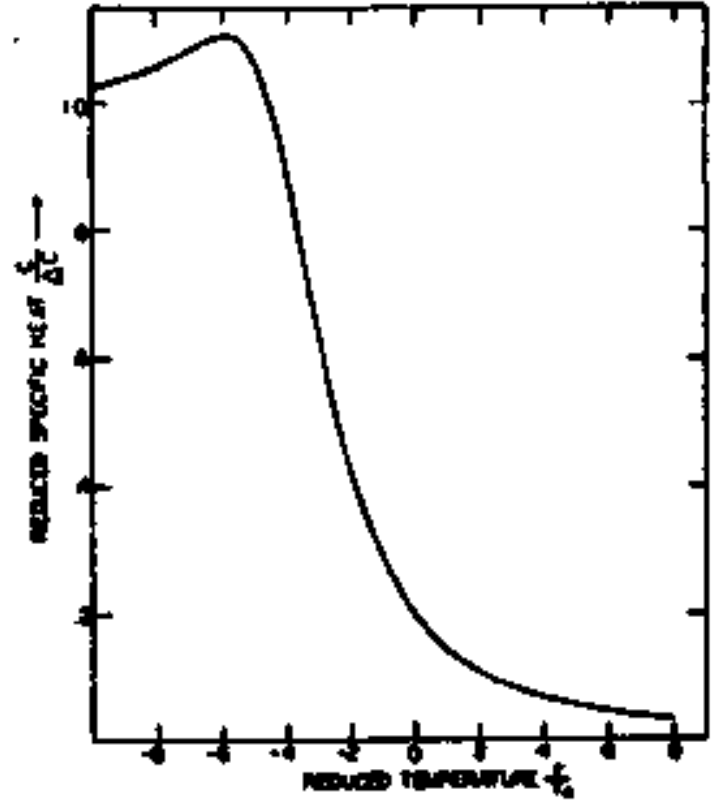

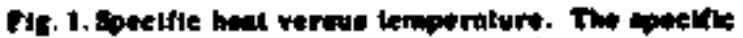

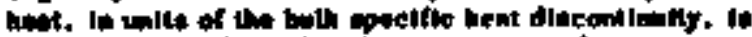

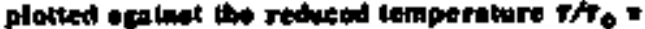

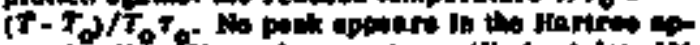

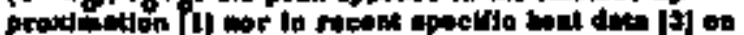

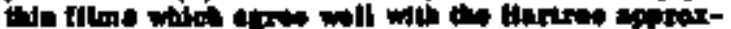
Inetion.

Figure 5. 〈from Ref. 12〉

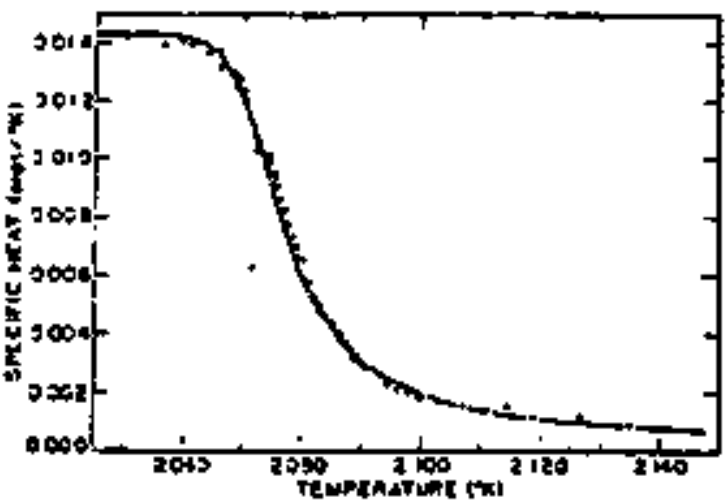

Fic. 1. Corposien of theor with axpetinent. The dots are the exporipental values of the specific heat of - $1350 \mathrm{~A}$ sample of $\mathrm{Bj} 5 \mathrm{~S}$, w plotted igatingt tempeseture. The solid cutve it the theoretieal earve of spocific hwat versuc tempetatut: determined by the equation $f(C, 0.0143) \times\left(0.0143^{\prime} C-1\right)-\ln \left(0.0143^{\prime} C-1\right)$ - 323.6 $T+659.3$.

Figure 6. (from Ref. 13) 


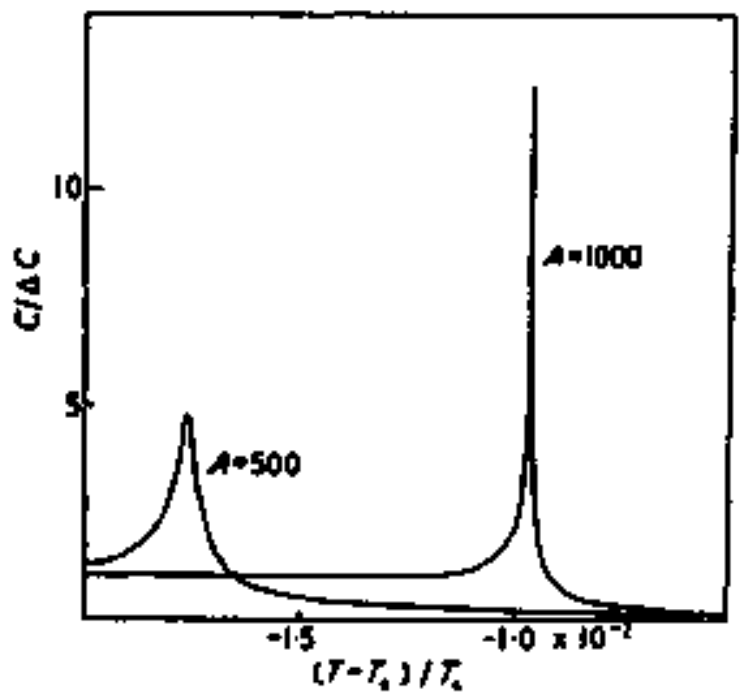

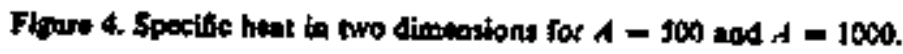

Figure 7. (from Ref. 14)

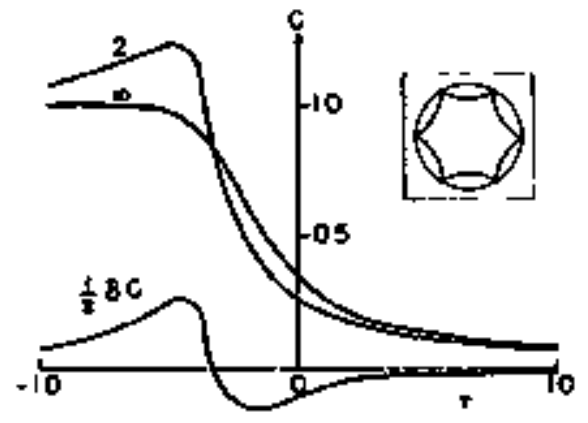

F1G. 1. Specifle heat $C$ of a two-dimensional Cinzbuig-Landau model versug reducod temperature $r \quad c$

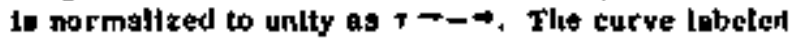
"w" ahows the opeciflc heat per component of an $\mathrm{N}-\mathrm{com}$ " ponent model In the Hactres Ifmit, n-m. The curse Iabeled "2" abows the flrst-order sereening spproviniation for a $=2$. The difterence betwean the two curves is yoC, the acreoning correction, nod te shown by the bottom cutre. The Feynnian groph oboun in the uppor right-hand Inact ds a typleul ring diagrum Included in the screcing correstion.

\section{F1 gure 8.}

Heat Capactity in 2-0 including screening approximation terms (graph \$2). (from Ref, ;5). 
to the B.C.S. value. The calculations involve different forms of a Hartree approximation. The work shown in Figure 7 includas the screaning terms in the Hartres approximation. The technique of extending perturbation theory far tnto the critical region, where it is expected to break down, is qufte risky. In fact, this method is questionable, even in conductivity work, where the agrement with experinent is quite good. ${ }^{6}$ As pointed out earller, the renomelization technique is the best approach. Figure 8 shows the results of using a renormalization approach, but due to the difficulty of the probtem, perturbation theory was used. The curve labeled "so" is the Hartree result equal to that of Figure 6 . But, by including higher order terms, additional contributions give the rasult labeled " $2 "$ " which shows a peak in 2-0. If one included even higher order terns, one might expect "cusp-like" feature to appear (at the peak of Fig. 6). This work also is in disagreement with the work of $\mathrm{ZM}$,

Thus, the heat capact ty shoutd exhibit a behavior similar to the excess conductivity above $\tau_{c}$ in 2-D, but its behavior near $T_{c}$ is not understood. One would expect the 3-D system to oxhib1t a singularity like that observed in other 3-D phase transitions (i.e.. LHe ${ }^{4}$ ). But due to the narrow width of the critical region in 3-0 $\left(\varepsilon_{c}\right)$ and the rather targe samples used, one would expect imhomogenieties to broaden the resuits ( $\varepsilon_{\text {inhom. }}>\varepsilon_{c}$ ). The 1-D prediction is an exact calculation, and because of the experinental difficultles, this problem has not been studied. 
A 0-D experiment (dust) might be posstble, but it is not quite understood theoretically. The 2-D problem is of greatest interest because of the different predictions. Some predictions are for a full phase transition and a divergence, as in 3-0. Other predictions are for no phase transition, but a definite nonmonotonic peaking to occur near $T_{c}$. Finally, other predictions indlcate no paking at all. Because of these predictions, the experiment was carried out on 2-0 system where one is interested in, 1) the behavior above $T_{c}$, where one expects a precursive nature, and 2) whether or not a peaking accurs in the transition to the B.c.s. Jump. 
Chapter 3. Previous Experimental Hork

The experimental work in measuring fluctuation effects in the superconducting transition is substantial. Some of the measurewents of conductivity on aluminue flims will be discussed in the appendix on sample preparation. However, there has been relatively little work done on look1ng for fluctuation effects in the hest capactiy. This is most Ifkely due to the difficulty of the experiment.

One of the early studfes of fluctuation effects was that of Cochran. 34 In making an analogy to the work on the superfluid transition of $\mathrm{He}^{4}$, he looked at the heat capacity of bulk pure superconductors very near their transtitions. F1 gure 9 shows the work of Cochran for the total heat capacity vs. temperature plotted for the bulk samples of Ta-11. The dots are the experimental data, and the dotted line and the contfnuous line are two theoretical curves. In analyzing his data, cochran used form for the heat capacity that had the bCS jump plus a diverging form for the butk sample $\left(\varepsilon^{-\frac{1}{2}}\right)$. It is shown below.

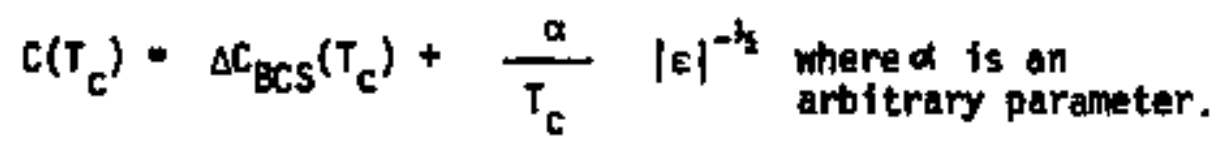

Then, because he was working in bulk samples, where one would expect an inhomgenfous system with multipie transitions, he formed an average of the individual hest capactities (eq. 11) assoctated 


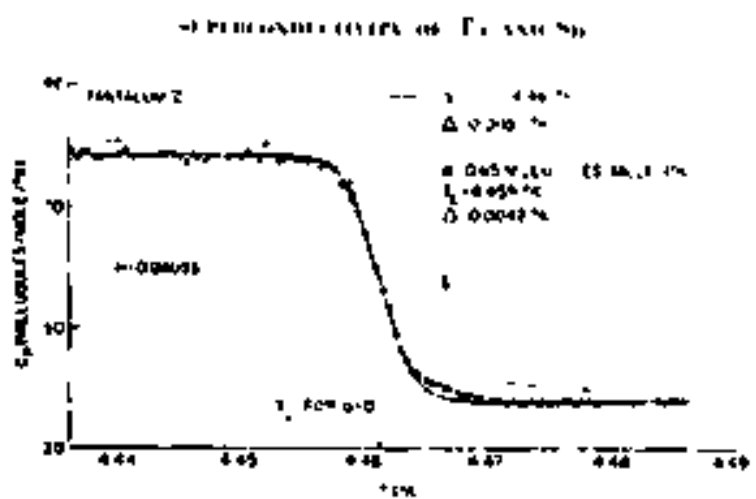

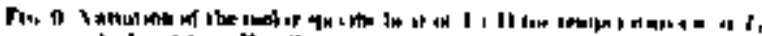

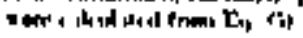

Figure 9. (from ref. "34)

46

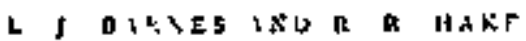

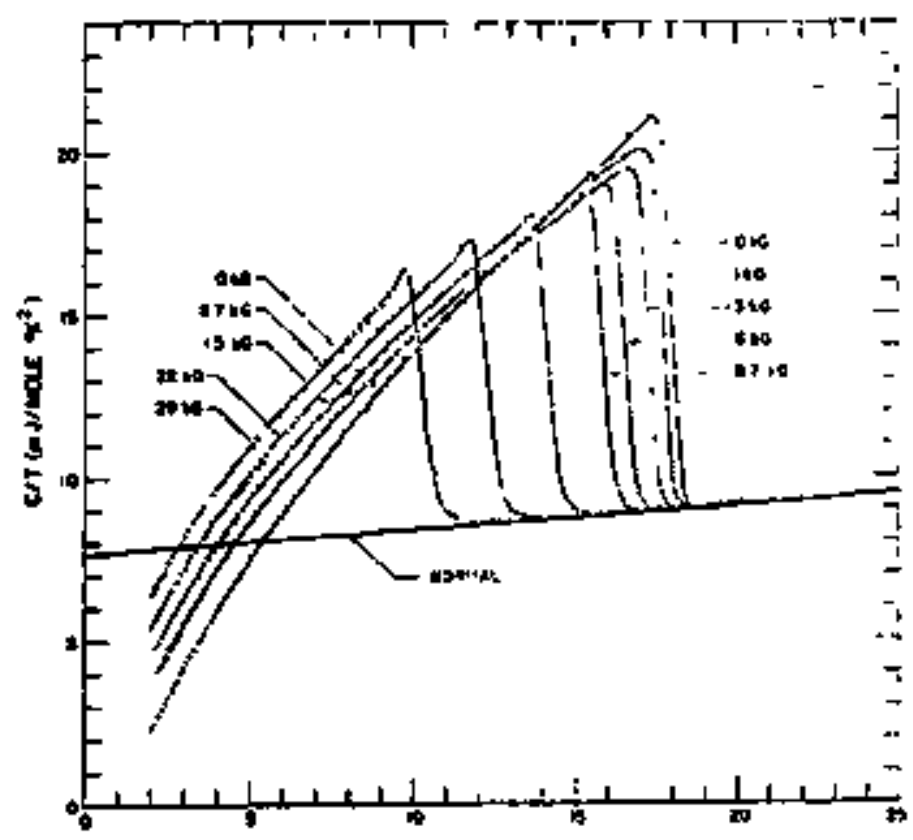

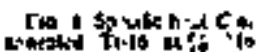

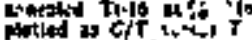

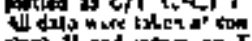

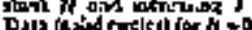

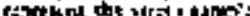
Dint

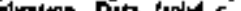

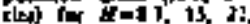

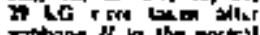

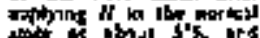

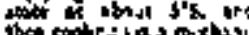

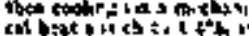
cont that cut Ior $H=1,3, B 14$ nor

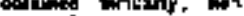

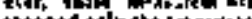

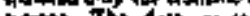
hront The ent in mather Thin Hod ine tontex to 1 t

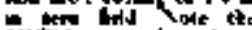

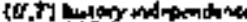

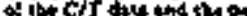

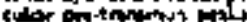

Figure 10, (from ref, 35 ) 
with each transition. This average was weighted about a MFT $T_{c}$ by a Gaussian-like function of the difference in transitfon temperature from the MFT value which is shown below:

$$
C(T)=\int_{-\infty}^{\infty} \frac{1}{\sqrt{\Delta \pi}}\left[\exp -\frac{\left(T_{c}-T_{M F T}\right)^{2}}{\Delta^{2}}\right]\left[\Delta C_{B C S}\left(T_{c}\right)+\frac{\alpha}{T_{c}|\varepsilon|^{-\frac{1}{2}}}\right] \Delta T_{C}
$$

His best fit to the data was for $\alpha=0$ and $\Delta=3.2 \mathrm{mK}$, which leaves out the precursor (the continuous line in figure 9). The rounding or precursive nature of the data in going over to the BCS form is most likely due to the inhomogenieties broadening the transition. Cochran piaced an upper limlt on the entropy associated with singutarity in the heat capacity of the matals studied $(T \cdot, \mathrm{sn}) a 5 \sim 10^{-3} \mathrm{~J} / \mathrm{mole} K$. Because of the expected width of the critcal region $\left(\sim 10^{-12} K\right.$ ) for bulk pure samples, one can conclude that Cochran observed only MFT effects in an inhomogentous system.

Wore recently, Barnes and Hake ${ }^{35}$ measured the heat capacity of bulk dirty samples of Ti. $.84^{\text {th }} .16$ in Targe magnetic fields. FIgure 10 shows their results of the total heat capacity (df vided by temperature) plotted vs, temperature squared. The narmal BCS discontinuities are apparent. But, one also notices the reduction in the transition temperature $\left(T_{C}\right)$ and the widening of the transition region with increasing values of applied magnetlc ffe]d. This is in agreentent with the magnetic fleid predictions of Lee 
and Shenoy presented earifier. One should notice the rounding or precursfye nature as the transition is approached, for the heat capacity maasurenents at hfgher magnetic fields. They also mention posstble fluctuation region as broad as $0.5 \mathrm{~K}$ existing for their samples. In a recent paper, Hassing, Hake, and Barnes 36 reanalyze their prevtously mentfoned work, using an extension of the previously discussed idea presented by Lee and shenoy, where the magnetic field induces a reduction in the dimensfonality of the system to onhance fluctuation effects. Using a Hartree approximation for the fourth order term, Hassing, Hake, and Barnes extend Lee and Shenoy's calculation through the transition region in a 1-D calculation of the heat capacity, Using a two parameter fit, their results are shown in Figure 11 , where the excess heat capacity is plotted vs. temperature. They attribute the disagreement between theory and experimant in the value of $\triangle C_{B C S}$ as being related to the choice of the momentum cutoff used in the calculation. A more obvious problem is the rather wide transition reglons observed $(\sim .4 \mathrm{~K})$, even for a zero value of the magnetic field $(\sim .1 k)$. They cannot account for this with their present theory.

The problems in looking for fluctuations in the heat capacity of a bulk system are shown in the two previously mentioned experiments. One has a very narrow transition region $\left(\varepsilon_{c}\right)$, and thus 11ttle experimental access to this region, even for enhanced

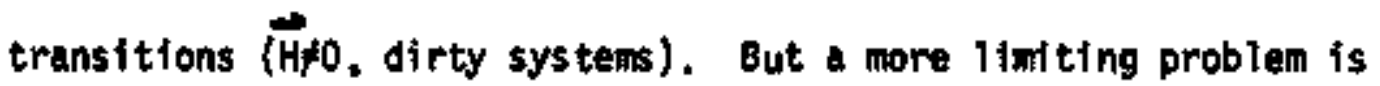




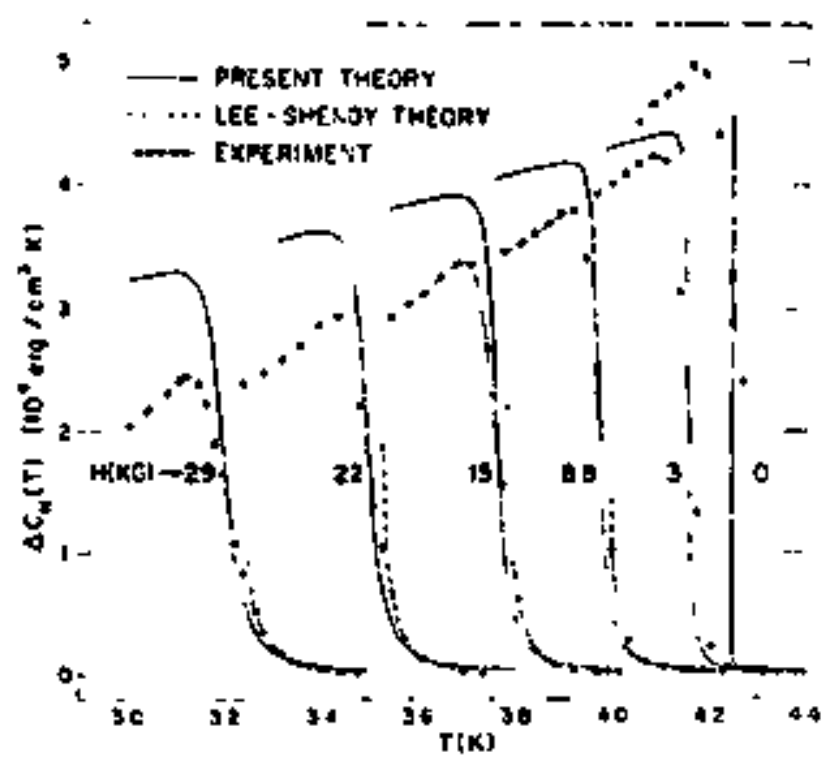

FIC. 1. Spectic heat difference, $\Delta C=C_{d}-C_{A}$, versta temperature $r$ tor varlous values of appled magnatic field $f$, as glven by the present theory, the theor: of Fat. 3, and the date of let. $s$. The dashed line (Leeshenos theoryl merges with the hexys golid line ipresont theory) for $T$ well above $T_{c 2}$ (Hn. The zero-H curvo clven in the present theory stops abruptly at $\Delta C=1.43 C$. at a slightly ablted zeco- $f$ transtion temperature $T_{\text {f }}$

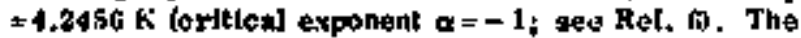
date polats are the abserved ouperconducting-state speetfic hest $C_{1}$ values milus the northil-state specluc heat $C_{1}=\gamma T+\beta T$, where $\gamma$ and $\beta$ were cketermined bs least-agrares fis for data taken in applled tields guffieient to quench superconductivity. All theoretseal ourves hlve been scaled so as to center the transltions at the experimental $T_{t-2}(t)$ as disidusutid in the text. The okporimentel talues of $T_{*}(H)$ for $H=30,24,13,8 . *$, 3, and o hG are, respoetixely, 3.316, J.509, 3.7ts.

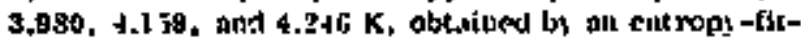

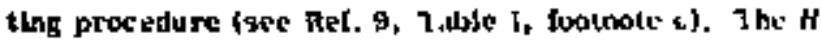

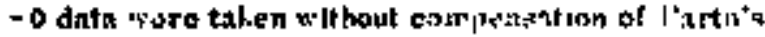
masnotse flelit,

\section{Figure 11. (from Ref, \#36)}

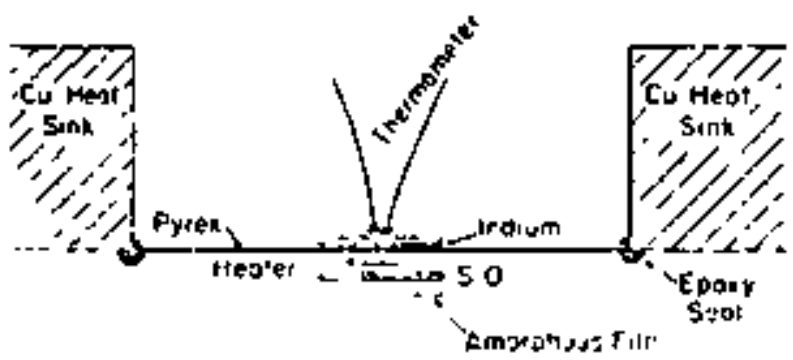

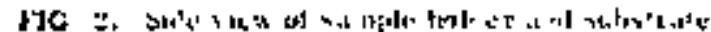

Figure 12. (from Ref. \#38) 
that of obtaining a bulk homogeneous sample. One can test for alloy concentrations, etc., but a more consistent measurement of the Inhomogeneities of the system is rather difficult (i,e., the sample resistance is al ready qut te small for $T>T_{C}$ in bulk system due to its geomatry). Thus, one would expect inthomogeneities to have large role in the broadening of the transition of bujk samples.

In a 2-0 system, where one is working with a thin $f i 1 m_{s}$ it is much easier to obtain and verify homgeneous samples, that one can use to study the heat capacity of the superconducting transition. Zally and Moche1 $1^{37,38}$ measured the heat capacity of a very dirty thin film. Their experimental configuration is show in Figure 12 . It consists of an amorphous BI.$^{5 \mathrm{~b}} .6 \mathrm{film}$ which is flash evaporated at $4 \mathrm{~K}$ onto the calorimeter. The calorimeter consists of $100 \AA$ constantan heater. separated from the film by a 5000 \& $510 \mathrm{film}$, and attached to the lower side of the glass plate. On the back side is a chip thermometer epoxied to a $5000 \AA$ In film. A.C. Calorimetry is used in measuring the heat capacity (see A.C. Caloriatetry Appendix). The low thermal dfffussivity of the glass crates a long thermal time constant from the calorimeter to the isothermal block, but also creates a long internal time constant for the calorimeter (1.e., the time constant to travel in the vertical direction of Figure 12), which limited the work to heating frequencles of les5 then $200 \mathrm{~Hz}$ (typtcally $2 t=60 \mathrm{~Hz}$ ). Figure 13 shows the experimental configuration in the plane of the glass 


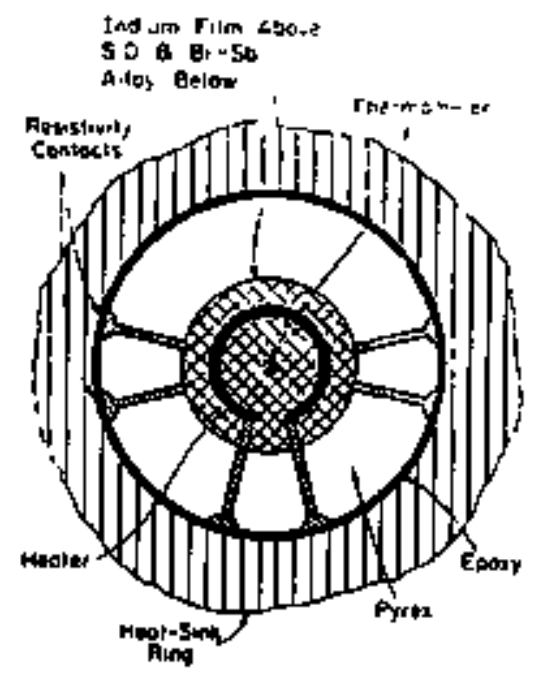

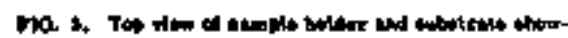

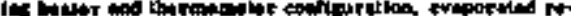

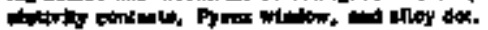

Figure 13. (from Ref. \#38)

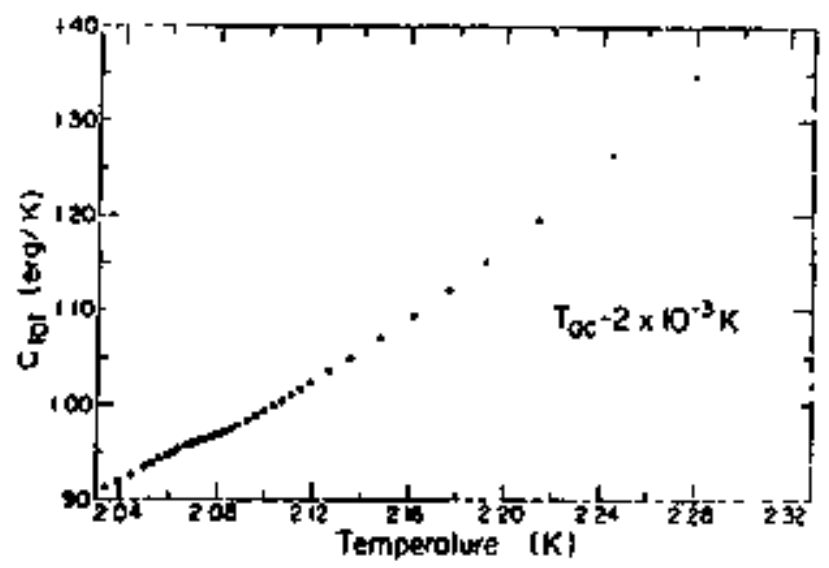

fTC $c$ Lnta for sivinpla bon la Mar baling bew the iselebt to hedt

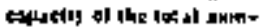
Din u Ietnperatu no

Figure 14, (from Ref, $\sharp 38$ ) 
plate (2-3 un thick). It also shows the non-unt form current. paths existing for the resistivity massurements of the fifm. It should be noted that these glass plates are actually glass "bubbies", so that there is some curvature associated wi th the film plane, and thus a non-uniformity in thickness. One should also not1ce the inductive nature of the heater configuration, which could lead to electrical coupling between the fitm and the Ge thermometer. Figure 14 shows the raw data of the total heat capacity and the calorimoter. The slight bump at $2.06 \mathrm{~K}$ is the superconducting trans 1 tion of the fiTm. To extract the electronic heat capacity from the raw data, Zally and Mochel used a four parameter fit that forced a zero excess electronic heat capacity for $T \gg T_{c}$ and a suparconducting excess heat capacity for $T \ll T_{c}$ where the excess is $C^{\prime}=C_{e s}-C_{e n}$. Then the excess heat capacity of the film was calculated from the equation below.

$$
C^{\prime}{ }_{f f 1 n} * C_{\text {tot }}-\left(A T+B T^{2}+C T^{3}+D T^{4}\right)
$$

8ecause the overall experiment was only accurate to 1 part in 1500. and the heat capacity of the calorimeter was $\sim 1 \mathrm{erg} / \mathrm{K}$, and the BCS Jump of the f1lm was $2.01 \mathrm{erg} / \mathrm{K}$, the measurentents on the film were accurate to only $10 \%$. In fact, they state $1 \mathrm{n}$ their paper that shuttilng the fitted parameters around can drastically change the form of the derived heat capacity of the flim. Their excess heat capactity is shown in figure 15, where the resistive transition is also shown. It should be noted that 


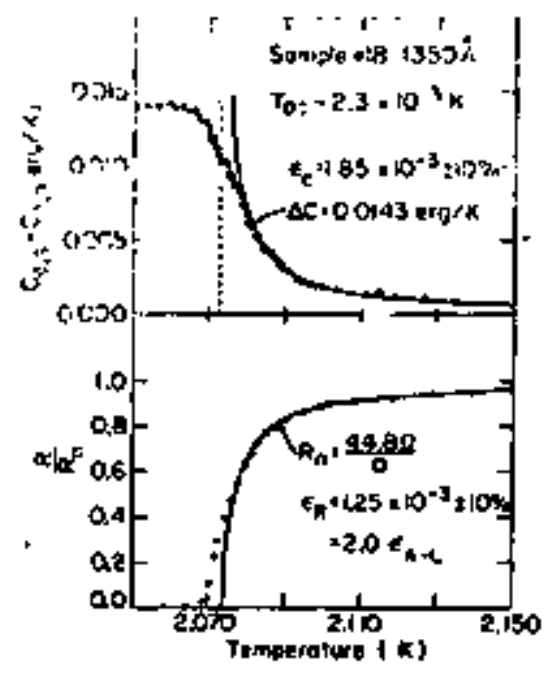

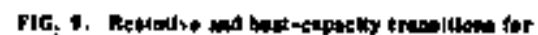

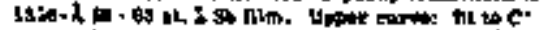

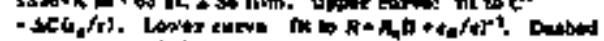
cume menertiols racule.

Figure 15. (from Ref. \#38)

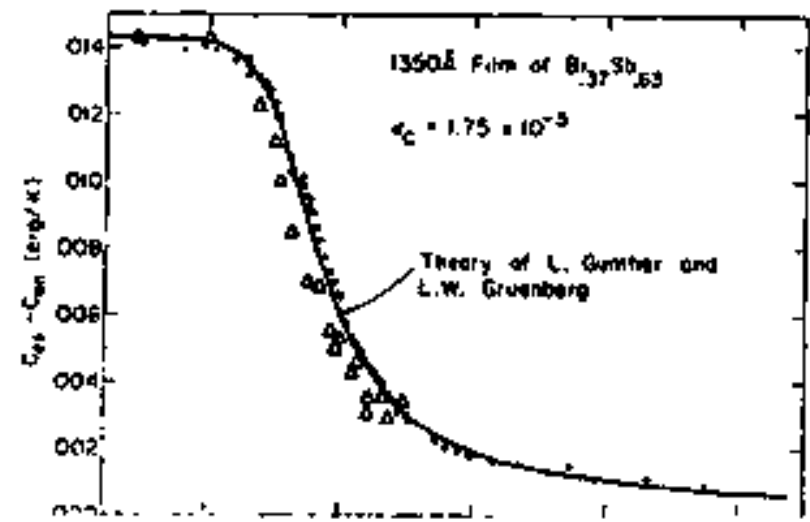

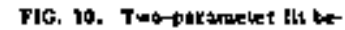

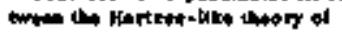

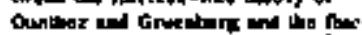

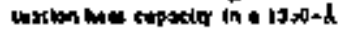

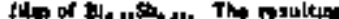

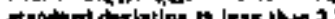
tint

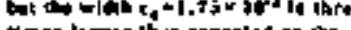

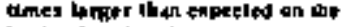

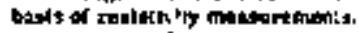

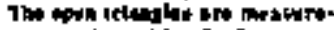

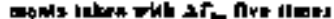

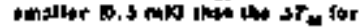

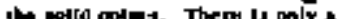

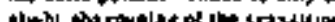

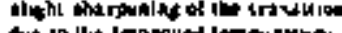
sue wo lut improved lemper rimax masoludion.

Figure 16. (from Ref. \#38) 
the circular gaonetry of the film presents problems in calculating ${ }^{N}{ }^{N}$ (the normal sheet resistance), but these problems are easily solved (see Sample Preparation Appendix). Also, the upper side of the calorimeter had $\mathrm{LH}_{\mathrm{E}}^{4}$ on tt to keep. the substrate cold during evaporation, and al though this was puaper out during the measurements, it never was totally removed. The resistive transition is off by factor of 2 from the A.L. theory. Since these films had a mean free path (l) of onty $.8^{837}$, one woutd expect quite good agreenent with the R.L. theory for the resistive transition. But this mean free path was only estfalted from the conductivity, and never independently measured. Because of the relatively few points taken in the resistive transition in the region where $A_{0} L$. should work, it is hard to accept the fact of this film's conductivity befing in agreement with a homogeneous transition in $A$. L, theory. F1nally, the results of Figure 15 are used in a two parameter fit to get the remarkable agreement to the thaory of Gunther and Gruenbers, shown in Figure 16. The true error bars on the curve are $\pm \Delta T_{A, C}$ on the $x$ axis, and $\pm C_{T O T} / 1500 \sim .0007$ ergs $/ K$ on the $y$ axis. This is on the order of the size of the triangles shown in Figure 16. Thus, one notices a consfderable scatter in the smaller amplitude heating, and a remarkable agreement for the larger amplitude heating, where the error bars should be larger. Zajly and Mochel claim not to have observed any peak in the heat capact ty as predicted, and to be in complete agreement with the theory of Gunther and Guenberg mentloned earlier. Their observation of an $\varepsilon^{-1}$ behavior in the heat capacity is consistent with 
fluctuation theory. Their results show the dffficulty of measuring the heat capacity of a thin f1]m by conventional methods. But, their use of a four parameter fit to extract the film's heat capacity is an arbitrary approach. When one adds to this another 2 paraineters to get agreement to the theory, the final results are not surprising at all. A true understanding of the heat capacity in 2-D w111 only come from results that are not forced to a particular form by multiple parameter fitting, and where control of the experiment is to a high eneough degree that one can readly distifss questions of electrfcal coupling and background $\mathrm{LHe}^{4}$ coning into the masurement. It is also necessary to make a final comment on their method of presenting data. In their first paper (ref. 37), they compare the widths of the resistive transition $\left(\varepsilon_{R}\right)$ and the heat capacity transition $\left(\varepsilon_{c}\right)$ to theory by plotting the ratio of these widths vs. the film's thickness. The data is badly scattered and only a weak conclusion can be drawn. In their second paper (ref, 38), they plot the same numbers as independent entities to the inverse power $\left(\varepsilon_{R}^{-1}, \varepsilon_{C}^{-1}\right)$ vs. filn thicknass. This somawhat obscures the differences between the numbers and gives a nore consistent view to the date. There is no physical reason ${ }^{*}$ why one plot is better than the other, and thus by choosing. the better "gicture", they are somewhat misleading the reader.

\footnotetext{
"Since $\varepsilon_{\varepsilon_{1}} \varepsilon_{R}{ }^{J}$, d being the film's thickness, it will cancel in a ratio pibt, and remain linear in an inverse plot.
} 
There have been no other experiments, to the author's knowledge, to measure fluctuation effects in the superconducting transition by measuring the heat capacity of a thin film. There is some work being done to measure the heat capacity of thin flims thet is worth mentioning. Greene, King, zubeck, and Hauser ${ }^{39}$ have used a very cleverly dest gned calorimeter to weasure the heat capactity of granular aluninum films. They employ - \$1]icon chip calortweter that has a heater and thermometer doped into it. The chip is suspended from an isothermal copper block by alloy wires to provide the correct tine constants for A.C. Caloriwetry. Thay shine a chopped laser bean $(26 \mathrm{~Hz})$ onto the calorimeter to provl de the A.C. heating. Measurements were made on a 25 ph thick aluminum film $(1.1 \mathrm{mg})$ sputtered onto the surface of the silicon chip. The film's grain size was on the order of $5000 \AA$ (as determined from SEM measurements) and $i$ ts heat capac1 ty transittion was widened due to inhomogeneous broadening. One can infer from thefr measurements that any singularities in the hest capacity would have to have an entropy of less than the $5 \%$ agreement they get between the superconducting and normal state entropies. They afso measured a free standing granular aluminum film of simf Tar thickness $(1.6 \mathrm{mg})$, with a thermocouple themometer spot welded to the film. This film gave rosults similar to the sputtered $111 \mathrm{~m}$. There is one other group 40 that is measuring heat capacitios of thin films that is not looking for fluctuation effects. They have constructed a calorimeter from Bj-Ag thermocouples and a luminescent diode with their films supported on a 
collodian substrate. There is a possibility of this schame being useful for fluctuation measurements in th1 $\mathrm{f} 11 \mathrm{~ms}$, but they have not reportod using it for such measurements.

Thus, the experimental work to measure fluctuation effects in superconductors by measuring the heat capacity in the transition region is sparee. The results of measurements made in bulk systens are hindered by problems in obtaining homogeníous samples and accossible critical regions. There are no reported heat capacity measurements in 1-0 (wires). The only work done in 2-D that is posstbly in a honogeneous system and in the lower dimenstonal 1hat (the 25 un films probably too thick) is that of Zally and Mochel. Although their work is in partial agreenent with theory, there are considerable doubts about. their abilities to observe such small heat capacities burled amidst the heat capacity of a relatively large calorimeter. Thus, the previous experimental work fimples the difficuitles of measuring the heat capact ty in the transition region of a supercondactor, and as yet, it has still left the theoretical understanding of the problem open to question. 


\section{Chapter 4. Experiment}

\section{Bas lc Method}

Because the conventional experimental approach to the measurement of the heat capacity of thin films (as carried out. by ZM) has inherent problems of tenperature resolution and sccuracy, unconventional techniques were used in this experiment. One unconventional feature was to use the superconducting film's resistive transftion as a non-11near thermioneter to measure the buperature of the film (first suggested by Ferrell ${ }^{6}$ ). The adventege of this method over the more conventional approach is that the thermometer in the calorimatric masurement is always in thenrial 1 equilibrium with the fitm, and it contributes no background heat capacity. A second unconventional feature was to use A.C. Calorfmetry by heating the film optfcally with a modulated light source (see Appendix $F$ ). There is thus no contribution to the heat capacity measurenent from a heater, and the heater and the film are also in thermel equilibriun for moderate A.C. heating frequencies (see Appendix F).

Figure 17 shows a res1stive transftion of a superconducting fi1m. The film has been calibrated against a temperature standard such that for any value of resistance $\left(R_{0}\right)$ of the film, one knows the corresponding temperature $\left(T_{0}\right)$ of the film. Then if the film is heated with a power $\dot{Q}_{a b s}$ at a frequency $f=1 / \tau_{\text {heat }}$, the ffla witl experience a temparature rise at in each cycle as a 


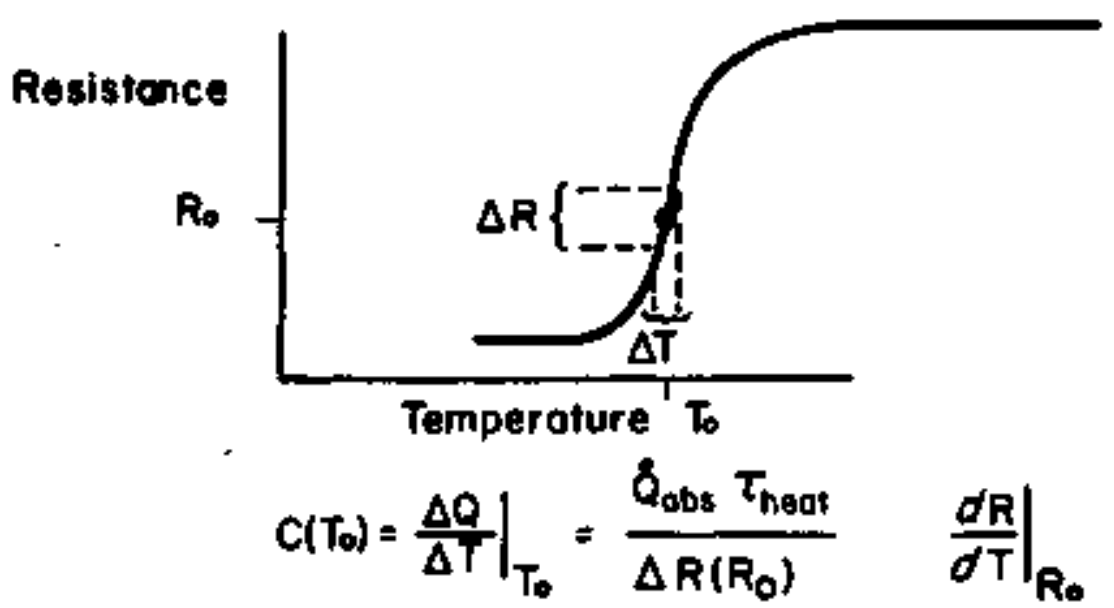

Figure 17. Resistive Transition being used as a

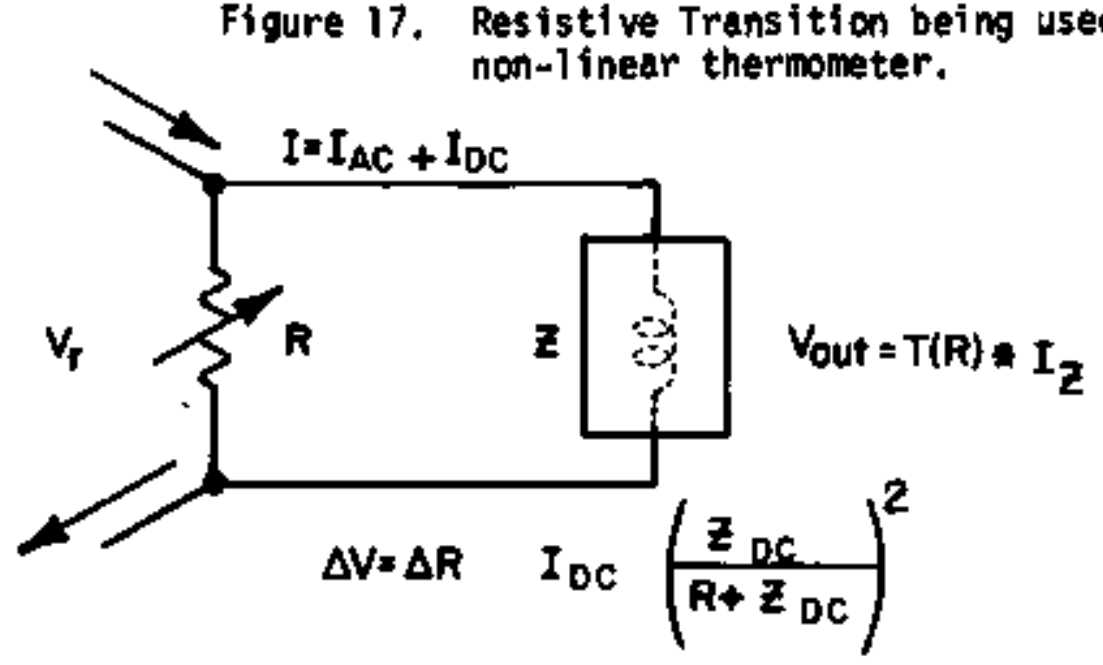

$R \propto V_{r}\left(I_{A C} R\right)$

$\Delta R \propto V_{\text {out }}\left(I_{D C} \Delta R\right)$

F1gure 18.

Equivalent circuit used in analys is of the signal resulting from the optical heating (vieat). With this analysis the voltage measured across the filan $V_{r}$ is proportional to the resistance $R$, and the vol tage measured across the transformer (vout) oue to the optical heating is a functional form of the changing resistance $\Delta R$. 
consequence of the energy put in during the cycle ( $\triangle Q=Q_{\text {abs }} \tau_{\text {neat }}$ ). Th1s will cause a corresponding rise in the resistance of the film $(\Delta R)$. The heat capacity of the film at temperature $T_{0}$ can then be deterutined by the equation in Fig. 17, where $d R /\left.d T\right|_{R_{0}}$ is the slope of the resistive transition at the point $R_{0}$. The disadvantage of this method is that the thermometer only works in the transition region, and because of the non-11near relation between $R_{0}$ and $T_{0}$, there ts a considerable roduction in sensitivity near the beginning and end of the transitfon (dR/dT is small). Also, because the optical absorption in the film is not known exactly $\left(\dot{Q}_{a b s}\right)$, the absolute magnitude of the heat capacity cannot be obtained. The use of this method is warranted because the present theoretical interest in the heat capacity of superconductor is $\mathrm{in}$ the transition region and the rather narrow predicted critical regions of temperature make high level of accuracy necessary. With this method, one sacrifices the obiltty to relate measurements to known behavior outside the transition region for the extreme sensitivity and thermal resolution possible. Because of the need for accurately characterizing the superconducting phase transition by measuring the conductivity and heat capacity of a film, the advantages of the unconventfonal methods used in this experiment justify their use. The basic procedure is to measure the changing value of the resistance of the fitn ( $\Delta R$ ) due to the optical heating $(\Delta T)$ as a function of the f1im's average resistance (R). which is varied through the whole transition by sweeping its temperature. This 
is done at different frequencfes and in an 1solated environment. Then thermal grounding of the film to a thermoneter is accomplished by adding He gas to the envinonment, and the film's resistance is cal1brated against temperature. Fron this data, the heat capac1ty can be calculated in principle, using the equation of Fig. 17 for $C\left(T_{0}\right)$. The actual procedure is more compl tcated, but only becausa of the problems of a non-1inear thermoneter. However, no other alteration or fitting scheme is used to transform the data to the heat capactity.

\section{Apparatus}

The calorimeter consists of a $1000 \stackrel{\circ}{A}$ aluminum film on a 2 mi1. mica substrate that is themally grounded. The f1lm is heated by ifght from square wave modulated laser (see Appendix B). measurements are made in magnetically, electrically, and vibrationally 1solated, thermally controlled enviromient (see Appendí $\mathrm{C}$ ). A. C. Calorimetry is used in the messurement (see Appendix F). The basic apparatus is shown below:

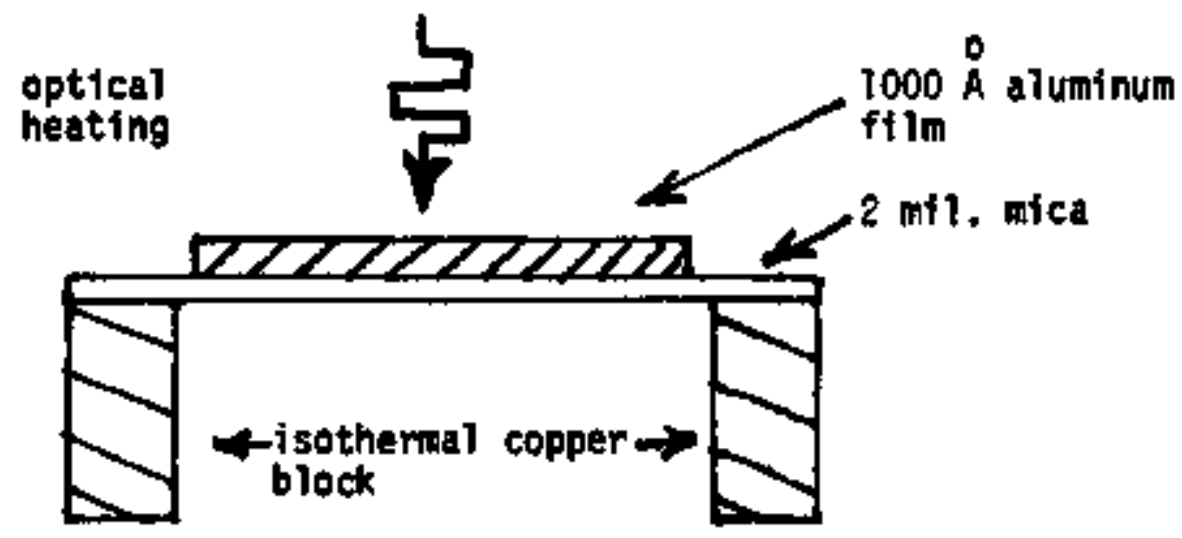


The film behaves as if it were fully self-supported (see Appendices $F$ and $H$ ). Thus, the fast therial response time of the film allows equilibrium measurements, even at high optical hoating frequencles (up to $20 \mathrm{KHz}$ ). An attempt was made to increase the sensftivity of the experiment at the ends of the transition by using a SQUID system, but this method was discarded due to problents that arose (see Appendix D). For more complete details, the reader is referned to the appendices.

\section{Equivalent C1reutt}

The f11m's reststance is measured in a dynanical way (see Appendix H) where the film is blased from a low frequency (41.5 Hz) constant current source (see Fig. 19) at low current densities of $1+5 \mathrm{Ncm}^{2}$. The phase sensisive detected voltage of the PAR-126 lock-in is scaled I inearly in resistance at a temperature where the film is far above the transition region ( $R=R N$ ). This scaling value for the resistance comes from a separate, four terminal A.C. bridge masuremant (see Appendix A). This voltage stgnat is then used throughout the experimient as the valua of the resistance. The out-of-phase contriburtion to the voltage is a small correction becouse of the low oparating frequenctes (see the discussion of the corrections in Appendix $A$ ).

The film is also biased from a constant D.C. current source at current densities of $10 \rightarrow 40 \mathrm{~A} / \mathrm{cm}^{2}$ (see Fig. 19). This current, in conjunction with the changing resistance $A R$, produces voltage 
signat $\Delta V$ that is aiso phase sensitive detected by a second lockin (PAR thR-8) with a reference that is coherent with the heating pulses $\Delta Q$. Because of the IOW signal level and source resistance, a transformar (see Fig. 19, the AM-1 is set for $Z_{\text {input }}=30 \Omega$, $Z_{\text {output }}=147 \mathrm{k}$ ) is in parallel with the ffim to increase sensitivity and $S / N$ ratio in the experfment. The combination of the transfonter and varying resfstance of the $f f l m$ creates a problem in determining the actual value of $\Delta R$ frow the measured signal at the lock-in. Tha probiem Is that the transformer in parallel with the film has - low D.C. Impedance, such that the O.C. current blasing the film is divided betwaen the film and the transformer. The film's changing resistance as the temparature is swept through the transition, couses the division of the current to change during the medsurement. Also, because the f $11 \mathrm{~m}^{\prime} \mathrm{s}$ resistance is changing, the transformer relates the voltage on the film $\left(Y_{r}\right)$ to the voltage going into the lack-in ( $V_{\text {out }}$ ) in a non-linear way.

To overcome th1s problem, a general equivalent circult is used to represent the real circult (note there are low pass filters in the circuft of fig. 19 that are not shown. See Appendix C). The equivalent circuit for the exporiment is shown in Figure 18, where the film is in parallet with on unknown Impedance $Z$ and is biased by an A.C. and a D. C. current. Voltages that are detected by the PAR ANR-8 are presentad by a transfer function $T(R)$ times the current $I_{z}$ in the impedance $Z$. Then, if the film is biased with a D.C. current, D. C. voltage $V_{r}$ appears across the resistance of the f1lm. Th1s voltage is given by the equation: 


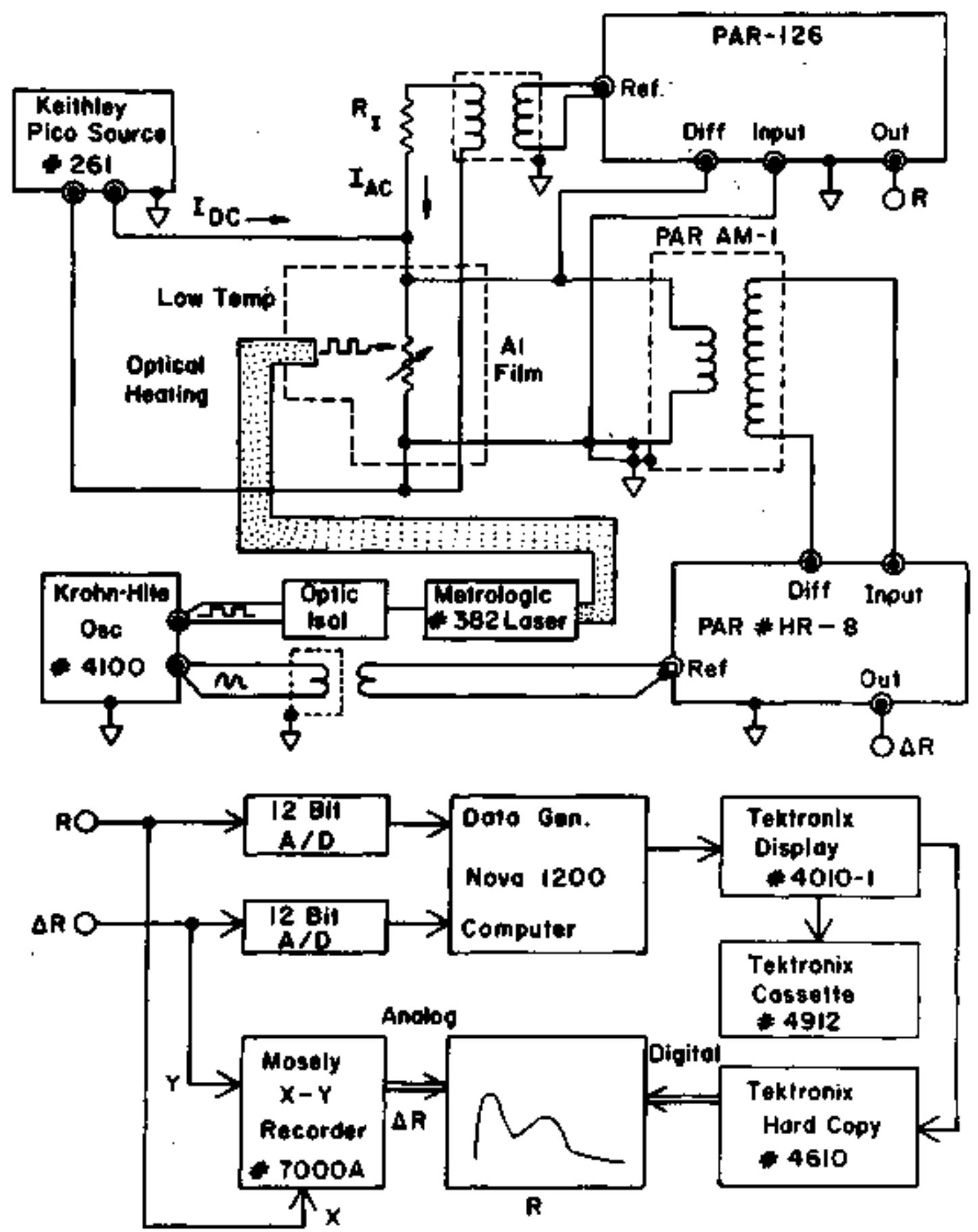

F1gure 19.

Biock df agram for electronic measurements including optical heating (vta fiber optics), renoval of electrical ground loops, and analog and digital recording and display systems. A direct comparison can be mado between all analog data and recorded digital data. AIl chass is grounds are to a copper bus bar held at a true ground. 


$$
V_{r}^{D C}=\frac{I D C Z_{D C}}{\left(R+Z_{D C}\right)}
$$

Now if $R$ is modulated by an anount $\Delta R$, in A.C. voltage appears across the whole resistor and is shown below.

$$
\begin{aligned}
& d V r=\left[\frac{I_{D C} C_{D C}}{\left(R+Z_{D C}\right)}-\frac{I_{D C} R Z D C}{\left(R+Z_{D C}\right)^{2}}\right] d R \text {, which reduces to: } \\
& \Delta V=I_{D C}\left(\frac{Z_{D C}}{R+Z_{D C}}\right)^{2} \Delta R .
\end{aligned}
$$

This is contrasted with the more common method of including the modulation $\Delta R$ as a voltage source ( $I_{D C} \Delta R$ ) in series with the resistance $R$. This is an incorrect mode1, since the voltage develops across the whole resistance $R$. Using the transfer function, eq. 15b becones?

$$
v^{\text {Hest }} \equiv \frac{T(R) \Delta V(\omega)}{Z(\omega)}=\frac{T(R) I_{D C}}{Z(\omega)}\left(\frac{Z_{D C}}{R+Z_{D C}}\right)^{2} \Delta R(\omega)
$$

$v^{\text {Heat }}$ is the output of the lock-1n detector measuring signals coborent with the heating. In order to determilne the two unknown functions of 9 . $16(T(R), Z(\omega))$, two calibration measurements are also made. The film is first biased with an $A_{+} C$. current at the frequency $w$ using the conflguration shown in Figure 19. The voltage developing across the film is given by: 


$$
v^{f 11 m}=\frac{I_{A \cdot C .}(\omega) R Z(\omega)}{(R+Z(\omega))}
$$

Then with the same bias, the signal is detected after passing through the transformer in the sane manner as for the heating signal. The result is shown below:

$$
v^{g a i n} \equiv \frac{t_{A, C}{ }^{(w)} R T(R)}{(R+Z(w))}
$$

Since the reststance of the source of these three signals ( $v^{\text {heat }}, \mathrm{v}^{\mathrm{ff} 1 \mathrm{~m}}, \mathrm{v}^{\text {gain }}$ ) can change during the measurements, there are phase shifts in the detected signal. Thus, the in phase and out of phase components of the complex signal are detected and contined to give:

$$
\left|v^{\text {signal }}\right|_{R}=\left[\left.\left(v_{0^{5}}^{\text {(gnal }}\right)^{2}\right|_{R}+\left.\left(v_{90^{\circ}}^{\text {signal }}\right)^{2}\right|_{R}\right]_{\text {detected }}^{1 / 2}
$$

Thus by measuring $\left|V^{\text {heat }}\right|$ as a function of $R$ (the 41.5Hz signal), and $\left|V^{f t \mid m}\right|$ as a function of $T$ (to be explained later), and $\left|V^{\text {gatin }}\right|$ as a function of $R$, eqs. 16, 17, 18, 19, can be combined to form the equations below:

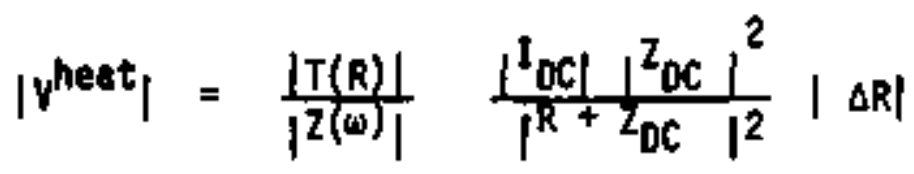

Since $Z_{\text {oC }}$ and $I_{D C}$ are rea], and $\left|V^{\text {film }}\right|_{T}$ is transformed to 
$\left|V^{\text {ftin }}\right|_{R}$, w $\left.^{\text {heat }}\right|_{R}$ can be wmitten:

$$
\left|v^{\text {heat }}\right|_{R}=\left|\frac{I_{D C} Z_{D C}{ }^{2}}{\left(R+Z_{D C}\right)^{2}} \Delta R\right|_{R} \frac{\left.\left(\left|v^{g a i n}\right|\right)\right|_{R}}{\left.\left(\left|V^{\text {din }}\right|_{T}\right)\right|_{R}}
$$

The signal $\left|V^{f 11 m}\right|_{T}$ is measured as a function of $T$ determined with Garmeniun resistor (see Appendix A, H). Then, foom the film calibration $(R(T))$ this can be transforned over to $\left|V^{f i l m}\right|_{R}$. Figure 19 shows how the data is recorded by a sampling of the analog signals, and a cross check is made of this digital sampling to the analog recordings.

The film's resistance is varied through the transition by driving the tempersture of the sample block (see Appendix $\mathrm{C}$ ) with an increasing power such that the film takes about 31 minutes to traverse the transftion region. The data is sapied every . sec.. such that 1020 pairs of data points are collected. Thus the following sets of data are recordad for one complete set used in a calculation.

$$
\begin{aligned}
& V^{\text {heat }}-\left\{R_{1}, v_{f}^{\text {heat }}(R)\right\} 0^{\circ} ; 90^{\circ} \begin{array}{l}
2 \text { sets } 1020 \mathrm{pt} . / \mathrm{set} \\
\text { at } 1 \text { pair } / \mathrm{pt} .
\end{array} \\
& v^{f i l e}-\left[T_{f}, v_{f}^{f i l m} \text { (T) }\right\} 0^{\circ} ; 90^{\circ} \begin{array}{l}
2 \text { sets } 1020 \mathrm{pt} . / \mathrm{set} \\
\text { at } \mathrm{pafr} / \mathrm{pt} .
\end{array} \\
& v^{\text {gain }}=\left(R_{1}, v_{f}^{g a i n}(T)\right) 0^{\circ} ; 90^{\circ} 2 \text { sets } 1020 \mathrm{pt} . / \mathrm{set} \\
& R(T) \text { callb. - }\left(R_{i}, T_{i}\right) 0^{\circ} ; 90^{\circ} 2 \text { sets } 1020 \mathrm{pt} . / \mathrm{set}
\end{aligned}
$$


At ten ASCII characters per/number (floating point notation), this results in about 1.5 Megabits ( $8 \mathrm{bits} / \mathrm{character}$ ) of stored data per run. The data is correlated in $R$ and $T$ to find $O R$ from equation 20b. The correlation process involves a search for the clesest point in the correlated parameter ( $R$ or $T$ ), and a limear extrapolation to obtain the correlated functional value. Oue to the large sampling grid, this linear approximation is quite good. The functional correlation equation is shown below where $f_{f}$ is the correlated value of $f_{j}$ at point $R_{i}$.

$$
\begin{aligned}
& \begin{aligned}
f_{f}\left(R_{1}\right)=f_{f}\left(R_{j}\right)+\frac{\left(R_{i}-R_{j}\right)\left(f_{j}-f_{j \pm 1}\right)}{\left(R_{j}-R_{j \pm f}\right)} \\
\text { where the }+ \text { sign is for } R_{f}>R_{j} \\
+\operatorname{sigh} \text { is for } R_{1}<R_{j}
\end{aligned}
\end{aligned}
$$

The equivalent circuit of Figure 18 can be made much more complicated (including filters, capacitors, etc.), but in the range of frequencies used $(2 \mathrm{~Hz}+20 \mathrm{KHz})$, the basic form of the signal equation (eq. 20b) renotins the same.

It was discovered that the discrete set of points for the two correction slgnals $\left|v^{\text {gain }}\right|_{R}$ and $\left|V^{\text {film }}\right|_{R}$ could be fitted to the functions shown below (notice that absolute values signs are omitted in the signel form of eqs. 17 and 18 : 


$$
\left.1^{\operatorname{san} n}\right|_{R}=\frac{a_{g} R Z_{g}}{\left(R+Z_{g}\right)} ;\left|v^{f i] m}\right|_{R}=\frac{a_{f} R Z_{f}}{\left(R+Z_{f}\right)}
$$

Here $\alpha_{g}, z_{g}, \alpha_{f}, z_{f}$, are appropriate values for the unknown Impedance $Z$ and $\alpha$ is a scaling parameter for a particular frequancy. $\left|v^{\text {gain }}\right|,\left|v^{\text {hast }}\right|,\left|v^{f 61 m}\right|$ are now measured in units proportional to the nomal resistance (scaled when $V_{r}$ is scaled to Ru), such that $\alpha$ is a dimansfonless paremeter. For a frequency of I KHz, the toro functions are equal $(\alpha=1.34, z=163 \Omega)$. For the vary low frequencles $(2 \mathrm{~Hz})$ the functions differ slightly in the valuas of the parametors $\left(a_{g}=3.05, z_{g}=22.1 \Omega_{0} a_{f}=1.34\right.$. $Z_{f}=[6 n)$. If a more complete model ts used for the impedance $Z(w)$, the difforence in the parameters can be explained for the Tow froquencies, but $t t$ in no way changas the final form of the heat signal in equation $20 \mathrm{~b}$. The value for $Z_{\text {oc }}$ was measured directly $\left(Z_{D C}=V\right.$ transformar $\left./ I_{D C}\right)$ and indirectly by a voltage division method. These results glve a value of $Z_{D C}=2.24 \pm .005 \Omega$. It should also be noted that the functions of 89.22 are almost constant curves in $R$ (due to the large $Z$ value), and the parameter velues are close to the masured values for the calibration $\left(a \sim \frac{I_{A C}}{\sqrt{2}}\right.$, $Z \sim Z_{\text {transformer. }} T \sim Z$ ) at the particular frequencies used. Thus, in this way, an analytical function can be used in the corrections of eq. 20b. At a frequency of $\mathrm{TKHz}$, the two functions $\left|V^{\text {ofin }}\right|_{R}, I\left(\left|V^{f i)}\right|_{T}\right) I_{R}$ cance $T$. Then the variation of the resistance of the film due to the heating is shown below. 


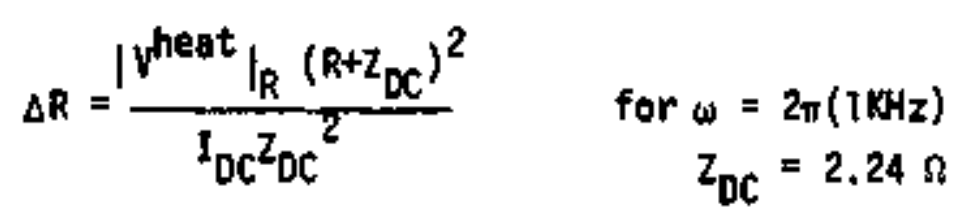

For lower frequenctes, the corrections do not cancel and are included explicitiy. but as mentioned before, the correction is small because of the Tow values of $R(u p$ to 300$)$, where the correction is of the form $\frac{(R+22)}{(R+16)} \sim$ constant. The understanding of this equivalent circutt was strengthened by modeling the experiment with a known variable resistor in the exact same circuit (fig. Ig) as the real film (even fncluding the filters). The resul ts have slightly different parametar values, but the exact same form. A furthor convincing fact of the model was that the signal $V^{\text {heat }}$ । was produced only from the optical heating (no ground loops, see F1g. 19). The D. C. current $\left(I_{D C}\right)$ could be changed by a factor of 5 and the signal would also change by a factor of 5 . The sign of the current could be reversed, resulting in a $180^{\circ}$ phase shift of the detected heat signal. The intensity of the hasting puises could be reduced by a factor of J0, and the resulting signal would atso scale. Finally, the light beam could be completely blocked, without altering any electrical circuit, and the signal would go to zero over the whole transition range.

Thus the changing resistance $\Delta R$ about the average value $R$ we to the heating is determined by the analysis presented above. The f1Im's calibration ( $R(T)$ ) is used to calculate the slope of the transition (dR/dT) and to trans form the measurements to tamperature. The slope is calculated using nearest neighbor derivative method 
and consistent averaging (see Appendix E). The energy detivered per pulse $(\Delta Q)$ is estimated (to be explained later) and the heat capactity of the film, using the equation of Fig. 17 is shown betow.

$$
c_{\text {tot }}(T)=\left(\left.\left.\frac{\delta_{\text {abs }} \tau_{\text {heat }}}{\Delta R}\right|_{R} \frac{d R}{d t}\right|_{R}\right) I_{T}
$$

At first, it was thought that the slope ( $\frac{d R}{d T}$ ) could be measured Just by measuring the heat capactity at very low frequencies ( $\mathrm{z} \mathrm{Hz})$ with a background of the gas, and then $C_{\text {tot }}(T)$ would be a slowly varying function, and the slope could be determined from $\Delta R$ and eq,24. Since the temperature range of the experiment ( $\Delta T_{\text {exp }}$ ) is very small relative to the operating temperature $\left(T_{\exp } \sim 1 . B K\right)$, the ratio of $\Delta T_{\text {exp }} / T_{\text {exp }} \sim 10^{-2}$. Over this small range, functions of $T, T^{3}$. exp (T) are constant to wfthin $5 \%$ error in the measured function. Thus the beckground heat capacity of low frequency measurement would Indeed be constant, and the $2 \mathrm{~Hz}$ measurement should be proportional to the derivative. But this was not the case, Indfcating that even at $2 \mathrm{~Hz}$, sizeable fraction of the heat capacity was due to the film. A final verification of the overall experfmental analysis was that the theoretical forms for the final results of conductivity and heat capacity could be fit to the data, and a back calculation to the raw data could be performed. The major features of the raw data agree quite well wf th the back calculation (sketched in the plot of fig. 19). Thus, although the analys is 
of the date was a compllcated process, the general method was sinple, and it involved no fitting of arbitrary parameters to the data. There was only slight snoothing done on the data In calculating the derivative (see Appendix E), and the final results were completely consistent with the rat data (the back calculation).

\section{Error Analys is}

Althoush there are many smaller errors in the experimental measurements, the major errors are due to the notse and drift of the signal [Vheat/ produced by the A.C. heating, and the noise and drift of the measurement of the resistance $R$. The jatter is especially serious for smatl values of $R$. These errors are predominantly larger than the intrinsic errors of the electronic equipment used in the measurements. The digital conversion of the analog signals does not reduce the accuracy of the measurement.

The data is sampled by unipoiar 12 bit analog-to-digital converters averaged at high speed by the computer software. The digital sampling has an overall accuracy of 2 parts in $10^{4}$. The analog signals are 1 imited by the drifts of the D.C. Output amplifiers. The output of the PAR HR- 8 lock-in, which is used to measure $T$ and $\left|v^{\text {heat }}\right|$, is accurate to 1 part in $10^{3}$ or about $5 \mathrm{mv}$, whichever is granter. The output of the PAR-126 lock-in which is used to measure $R$ is accurate to I part in $10^{4}$ or I $\mathrm{mv}$, whichever is greater. The modulated 11 ght source is quite stable over short times, but it has an overall drift of about 10\%. All reference currents are accurate 
to better than 1 part $\ln 10^{3}$. The correction functions $\left|v^{g a t h}\right|$ and $\left|v^{f 1}\right| m \mid$ are measured and fit to better than within $2 \%$ of the analytic functional forms mentioned earlier.

The tomperature is physteally stable to $10 \mu \mathrm{K}$ and by the use of the off-null bridge measurement (see Appendix A) of the Gormanium resistance thermometer, a $10 \vee$ signel of the HR-B corresponds to batween 20 and $100 \mathrm{mK}$. The noise of the HR-8 is then transformed through the film's calfbration to an equivalent temperature error of between 20 and $100 \mu K$. It should be emphasized that this exporiment does not require an absolute temperature measurement but only short-term temperature resolution between neighboring points. The accuracy of sampled temperature data is thus limited only by the short-term physical fluctuations which are of the order of $10 \mathrm{H} \mathrm{K}$. The resistance of the film is measured as a scaled voltage, so that an orror in the sampled resistance values corresponds to an error in the scaled voltage as a percentage of the maximem resistance of the f11m, RN. Since the resistance is measured with the PAR-126, this makes the error in the resistanca to be less than .1\% of RN, As for the tamperature measurement, the short term resolution of $R$ is govarned by the noise in the resistance masurement coming from the lock-In and from thermal fluctuations. The thermal fluctuation noise is the larger of the two and is on the order of $\mathrm{dR} / \mathrm{dT} \times 10 \mathrm{~W}$, which vartes from $30 \mathrm{~m} \Omega$ to less than $1 \mathrm{~m} \Omega$ depending on the value of $d R / d T$ for the particular point in the transition. The time constants used on the lock-ins represent an averaging of between 6 and 12 nefghboring points for the $R, T$ measurements and $\left|V^{\text {heat }}\right|$ 
measurements respectively. There can be considerably less averaging in regions where the measured quantities are rapidly varying.

Thus, the major errors in the experiment are in the $\mid V^{\text {Heat }}$ signal, which reflects the fluctuations in the light intensity, and in the determination of the film's resistance and its derivative, with respect to temperature, in regions where $R$ is small. These contribute to the determination of the change in resistance $\Delta R$ from the optical heating. The errors in the biasing current $I_{D C}$ and in the value for the D.C. transfomer impedance $Z_{D C}$ are small contributions. In Figure 20 is shown the raw date used in the calcujation of the heat capacity for run 338B. Figure $20 \mathrm{~d}$ is the raw data for the signal | viat $\mid$ as a function of the film's resistance for an optical heating at a frequency of $1 \mathrm{KHz}$. The arrow indicates the value of resistance at which the signal to noise ratio ts unity, and hence the data has no meaning after that point. The fluctuations in the signal are confination of real nolse and fluctuations in the derivative of the resistance with respect to temperature ( $d R / d T, 500$ Appendix E). Figure 200 shons the function which corrects the $\left.\right|^{\text {lieat }}$ | signal due to the changing D.C. bias current resulting from the changing value of $R$. These two functions used in calculating $\Delta R$ from eq. 24 give a total error in the determination of $\Delta R$ of botween 2 and $8 x$, dapending on where in the transition the valuo Is determined. Figurts $20 \mathrm{~b}$ and $20 \mathrm{~d}$ also reflect the effect on the raw data (F1g. 20d) of the decrease in the biasing current as $R$ increases. Figure $20 \mathrm{c}$ shows the resistive transition of $\mathrm{film} \$ 38$ in a $T$ vs, R plot. Figure 20a shows dR/dT of Figure $20 \mathrm{c}$ plotted vs. $R$ 
on the same scale. The method of deterwining $\mathrm{dR} / \mathrm{dT}$ was outlined earlier. The region of meaningful ||$^{\text {Heat }} \mid$ data is indicated and it is smaller than the region of valld $\mathrm{dR} / \mathrm{dT}$ data. This region is reflected in F1gure 20c to indlcate the total range (points $P^{2}, P^{\prime}$ ) in temperature of valid data. The percentage error in the value of dR/dT varties from 5\% to $10 \%$ and is largest near the endpoints of the transftion.

The calculation of the heat capacity uses the equation shown in Figure 20. The major errors are in the values of $\left|v^{\text {Heat }}\right|$ and $d R / d T$, which are given above. These values result in between 7 and 18\% overall error in the value of the heat capacity. The error in temperature is considarably smeller, since it is just a reflection of the error in $R$ transformad to temperature and amounts to between 20 and $100 \mu \mathrm{K}$, as stated earlier. Ffgure 21 shows the heat capacity calculated from the raw dato of Figure 20 displayed over the temperature range of valid data. The errors in this calculation are reflected in the scatter at the higher temperatures, where the

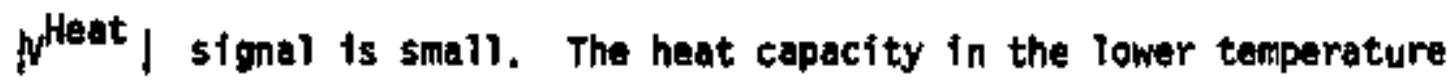
region increases above constant value with decreasing temperature. This effect will be discussed in the next section. The rise in heat capacity occurs in the region where $R$ is small, and the errors of $d R / d T$ are large, but the effect is significant despite the overall error of between 7 and $18 \%$. Since the teriperature resolution is considerabiy more accurate than the resolution in heat capacity, the monotonic behavior of the heat capacity in this region fodicates that over short regions, the error in this heat cepacity measurement 
Figure 20.

Row data used in determination of heat capacity. a) is the digital derivftive of the resistive transition of the film $\mathrm{c}$ ), plotted vs. the film's resistance $R$, b) is the correction to the heating signal d) due to the transformer in parallel with the fijm. The arrow ind teates the timit of valid data in d) and is reflected in figure c) to show a range in temperature $(P, P r)$ of val id data. The themal excursion due to the A.C. heating ts shown below.

$$
\Delta T \quad I_{R}=\left.\frac{\left|v^{\text {heat }}\right|}{(d R / d T)\left(\frac{Z_{D C}}{R_{D C C}}\right)^{2}}\right|_{R}
$$

For the data shown this is on the order of $1 \mu K$. The heat capacity is then given below.

$$
C=\frac{\dot{Q}_{a b s} \frac{d R}{d T} T_{\text {heat }}}{\left|v^{\text {heat }}\right|}\left(\frac{Z_{D C}}{R+Z_{D C}}\right)^{2}
$$



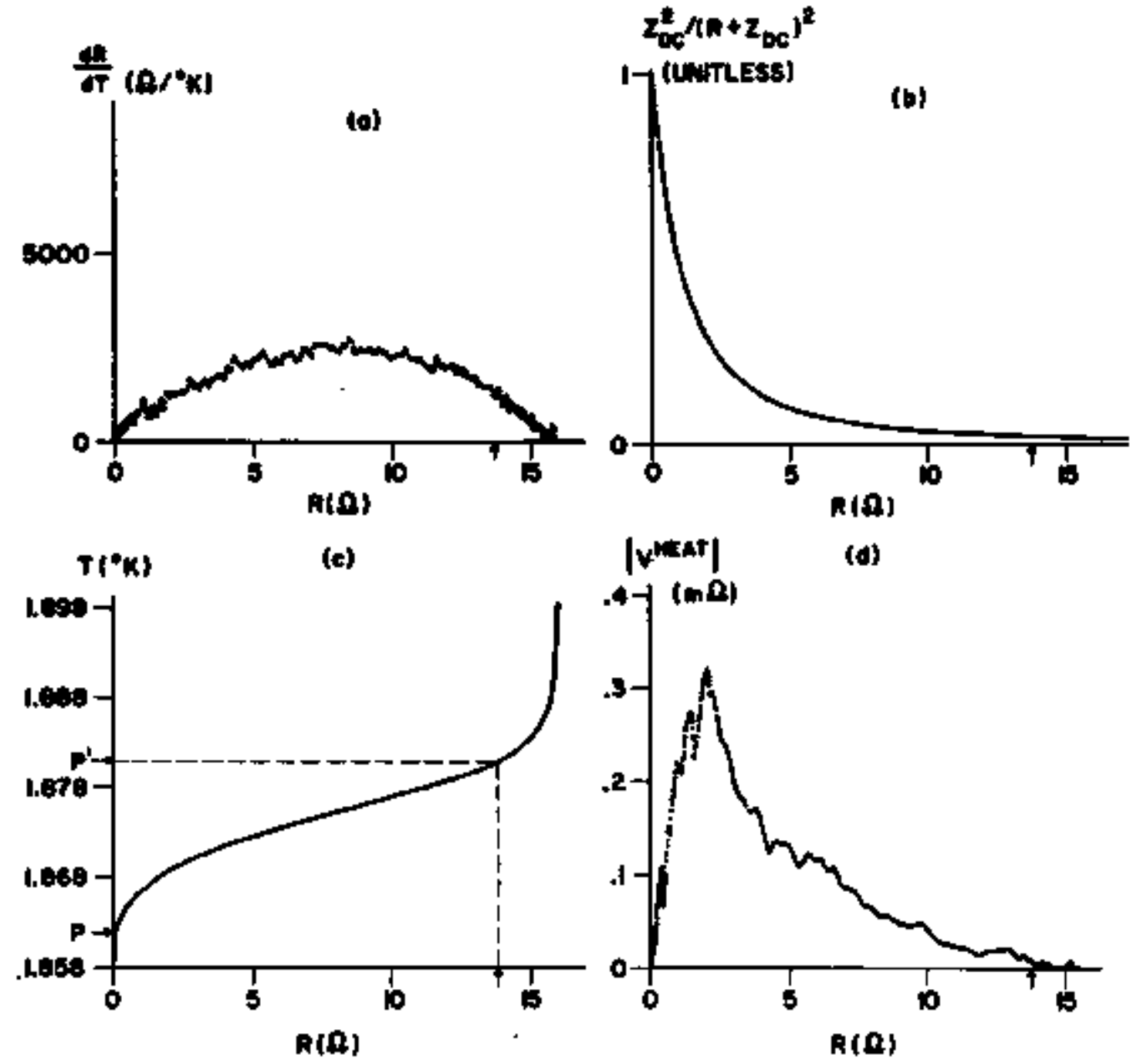

Flgure 20. 
is smalter than 7 to 18\%. The inset of figure 21 shows a semi-log plot of the logarithm of the excessheat capacity vs. temperature over a sma1ler range in temperature. This suggests that the total heat capactty may in fact, be diverging logarithmically. The excess conductivity of this f1lm also had the same slope and linear behavior on a logarithmic plot in this region.

The heat capacity is platted in relative units because ondy an approximate determination of the optical heating power $\dot{Q}_{\mathrm{ABS}}$ was made. Two methods were used in this determination. The first was to use an optfical detector in place of the film to measure the amount of light shining down on the region the film occupies in the expertment at low temperatures (see Appendix $B$ and Appendix C). Taking this value and the estimated light cone distribution and optical absorption of the $\mathrm{f} 1 \mathrm{Im}$, the valtie of $\dot{Q}_{A B S}$ was estimated at $2 \times 10^{-11}$ watts. A more accurate method was to stabilize the $f i 1 \mathrm{~m}$ in a sensftive region of the superconducting transition (dR/dT large) with the laser off and measure the temperature rise due to turning the laser on for a short period of time. This sane rise in temperature could also be produced by increasing the joule heating of the film by changing $I_{D C}$. If this is done fast enough ( $\sim 1$ sec.). it gives an adiabatic determination of the power absorbed $\dot{\theta}_{A B S}$. The value of $\dot{Q}_{A B S}$ estimated this way was between $5 \times 10^{-13}$ and $2 \times 10^{-12}$ watts. As this mothod of determining $\vec{Q}_{\text {ABS }}$ should be more accurate than that method explained earlter, one takes the value of $\dot{Q}_{A B S}$ to be on the ordar of $10^{-12}$ watts. Using the equation of Figure 20 , one then finds the value for the heat capecity of Figure 21 in the flat region to be 


\section{RUN *38B}

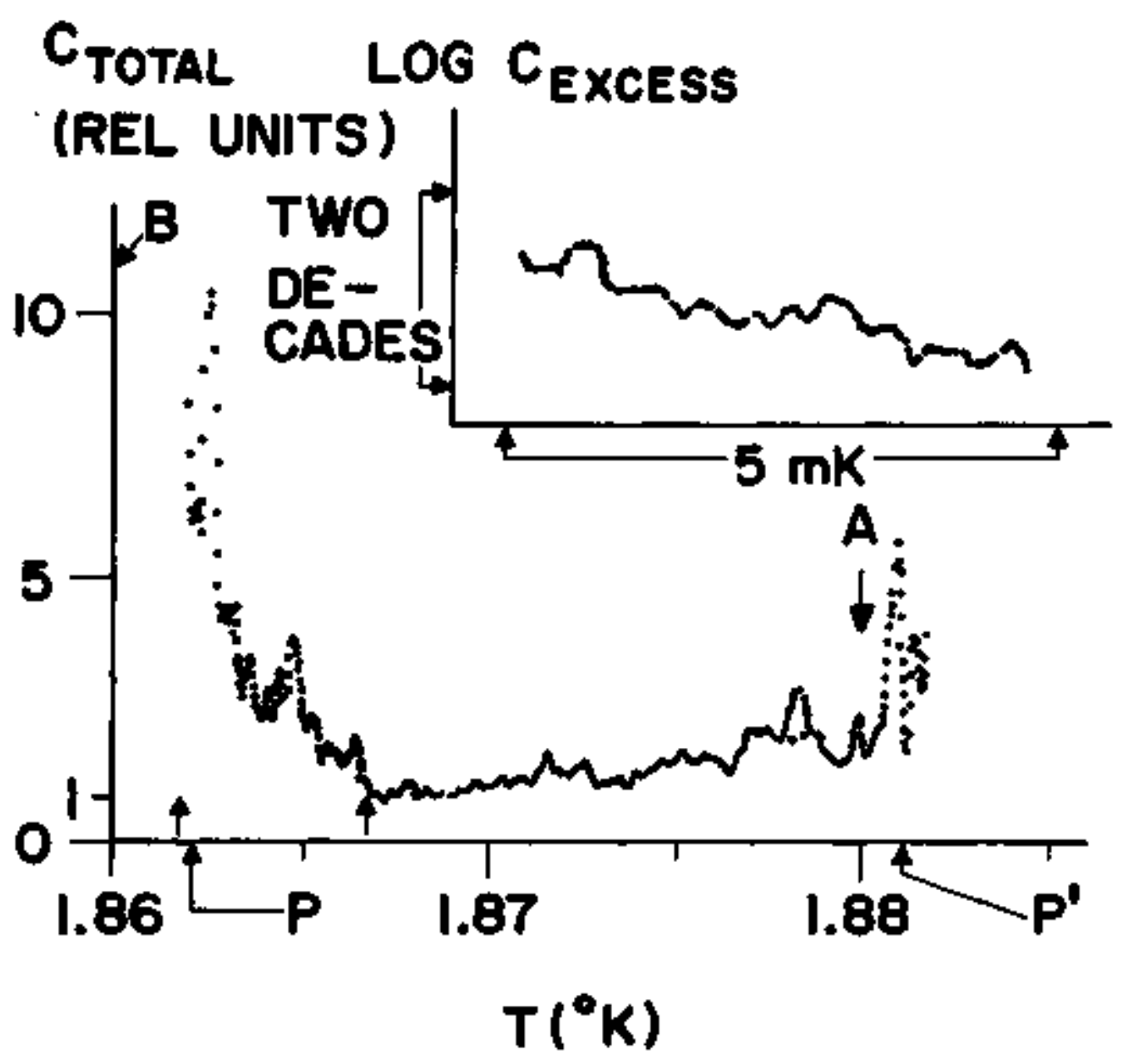

Figure 2 i.

Plotted is the total heat capacity of Sample $38 \mathrm{~B}$ for a $1 \mathrm{KHz}$ optical heating frequency. The points $P$, $p$ indicate the region of vatid data. The dats between the arrows is plotted in the insert as an excess heat capacity ( $C_{\text {excess }}=\mathrm{C}_{\text {total }}-\mathrm{C}_{\text {background }}$, where $\mathrm{C}$ background is the linear region at higher temperatures). The insert is - Iogarithmic plot of the excess heat capacity and shows a linear behavior over the region indicated by the arrows. Point $A$ is the MFT $T_{c}$, and point $B$ is the temperature for $A=0$. 
on the order of that calculated for the film in Appendix $F$. If the heat capacity in the flat region of Figure 21 includes a contribution from part of the substrate, then what one has calied an obsolute daternination of the film's heat capacity is oniy an upper Iimft. In Appendix $F$ the maximum percentage contribution to the heat capacity by the mica is estimated at about. $50 \%$ of the total heat capacity when the latter is measured at $1 \mathrm{kHz}$.

In conclusion, the errors of the experiment largely are the result of the "noise" in the detemination of $\mathrm{dR} / \mathrm{dT}$ and in the raw date ( $\left.\left|V^{\text {Heat }}\right|\right)$. Th1s error is between 7 and 18\% overal1, but it is considerably better over short ranges. The absolute determination of the total heat capecity measured is on the order of the values expected for the film, and the percentage of the messured heat capacity contributed by the film is between 50 and $100 \%$. 
Chapter 5. Oata Analysis and Discussion

General Features

The properties of the films studfed are outlined in detail in Appondix $H_{*}$ Measurements were corried out usfing experimental. methods discussed earlier. All data discussed in this section was taken in zero magnetic field unless explfcitly noted, and at an optical hoating frequency of $\mathrm{KHHz}$ ( $\tau_{\text {heat }} \cdot 10^{-3}$ sec. $)$. The films we made by slow evaporation of aluminum in varying pressures of $\mathrm{O}_{2}$ gas onto cleaved mica substrates. Particular values for the preparation parameters of the films discussed are listed in Tabie 3 . The flims are generaliy quite granular (average grain size $\sim 400 \mathrm{~A}$ ), with a significant range in grain size. They also have short mean eree paths $\left(l_{\mathrm{eff}}\right)$ as determined from the nomal state sheet resistance $R^{N}$ g 1isted in Table 3. An 1ndependent determination of $2_{\text {eff }}$ was done In an appl led magnetic fleld (see Appendix H), which was in agreement with that calculated from $R_{D}^{N}$. The transition teniperatures determined from the resistive transitions are on the order of 1.86K. The constants 11 sted in Table 3 show a reasonable consistency in the physical nature of the filns discussed, with the exception of sample $* 30$ (run $* 301$ ). This film was evaporated with a larger $\mathrm{O}_{2}$ background. Its resistive trensition was arasticalty affected by the anount of D.C. biasing current used in the experinent. Increas1ng this current resulted in "kinks" appearing in the transition. By increasing the A.C. biasing current used in the 
Fable 3. Physical constants for Films shown

\begin{tabular}{|c|c|c|c|c|c|c|c|c|}
\hline $\begin{array}{l}\text { Sample } \\
\text { (Run })\end{array}$ & $\begin{array}{l}\text { Preparation } \\
\text { (gas during } \\
\text { exp.) }\end{array}$ & ( & $\begin{array}{l}\ell_{\text {eff }}^{* *} \\
(\AA)\end{array}$ & $(\Omega)$ & $\tau_{0}^{\text {A.L. }}$ & $\tau_{0}^{f i t}$ & $\begin{array}{l}r_{c}^{M F T} \\
(K)\end{array}$ & $\begin{array}{l}T_{0}(p t . B) \\
(K)\end{array}$ \\
\hline 301 & $\begin{array}{c}2 \times 10^{-5} \text { Torr } \\
0_{2}\end{array}$ & 1090 & 13 & 2.77 & $4.21 \times 10^{-5}$ & $7.04 \times 10^{-4}$ & 1.829 & 1.778 \\
\hline${ }^{\star} 368$ & $\begin{array}{l}1 \times 10^{-7} \text { Torr } \\
\mathrm{HO}_{2}\end{array}$ & 1040 & 14 & 2.63 & $4.00 \times 10^{-5}$ & 2. $32 \times 10^{-3}$ & 1.880 & 1.860 \\
\hline 388 & $\begin{array}{l}2 \times 10^{-7} \text { Torr } \\
\text { No } \mathrm{O}_{2}\end{array}$ & 1170 & 53 & .644 & $9.78 \times 10^{-6}$ & $9.50 \times 10^{-5}$ & 1.898 & I. 860 \\
\hline 39 & $\begin{array}{l}2 \times 10^{-7} \text { Torr } \\
\mathrm{HO}_{2} \mathrm{O}_{2}\end{array}$ & 1190 & 23 & 1.46 & $2.21 \times 10^{-5}$ & $7.50 \times 10^{-5}$ & 1.843 & 1.814 \\
\hline & $H=21.800$ & \multicolumn{4}{|c|}{$\begin{array}{l}\text { The fleld lowered the temperature of } \\
\text { the transition and broadened the } \\
\text { oversil transition. This meas ure- } \\
\text { ment gave a value of } \xi(0) \approx 440 \mathrm{R} \text {. } \\
\text { See Appendix } \mathrm{H} \text {. }\end{array}$} & $7.38 \times 10^{-5}$ & 1.827 & 1.758 \\
\hline
\end{tabular}

Hote: The width to length ratio was between 3 and $4 \times 10^{-2}$ for these films. The evaporation rate was between 25 and $35 \mathrm{~h} / \mathrm{sec}$. The ratio of the resistance at $300 \mathrm{~K}$ to thie nomal res istance was between .8 and 1.2 . Point $A$ in the heat capacity figures is the MFT $T_{C}$. Point $B$ is the temperature for $R=0$.

*Double transition. \#Using the relation $2_{\mathrm{eff}} \frac{4 \times 10^{-12} \Omega \mathrm{cm}^{2}}{R_{\square}^{N} \mathrm{~d}}$. See Appendix $H$. 
experiment to a level equal to the D.C. current, these "kinks" could be dramatically reduced. When the "kinks" were removed, the overali transition was slightly different from the transition measured with a current less than that whtch would affect the resistive features of the transition (on the order of $10^{-6} \mathrm{~A}$ ). This effect is discussed in more detail in Appendix $H$.

The heat capacity measured for film \#3B is shown in Fig. 21. The andysis that led to this result is discussed in an earlier section. Over the temperature interval of the data plotted in Figure 21, functions exhlbiting $T, T^{3}$, and $\exp (T)$ are constant to Within $5 \%$. Thus over the range from about $1.867 \mathrm{~K}$ to $1.8 \mathrm{BK}$, there is a contribution to the total heat capacity that is constant. The electronic contribution of the f1]m's heat capacity is $80 \%$ of the total heat capacity of the film (see Appendix H, F) and the estimated amount of background heat capacity (from the mica, see Appendix F) is between 0 and $50 \%$ of the total heat capacity measured. Thus, in the constant region of the total heat capacity in Figure 21, the electronic heat capacity of the film represents between $80 \%$ (no mice contribution) and $40 \%$ (50\% mica contribution) of the total measurement. This means that the normal state heat capacity, defined os $C_{n}=C_{\text {electronic }}$ for $T>T_{c}$, is between .4 and .8 relative units on the plot of Figure 21. One would expect that the B.C.S. discontinuous jump in the heat capacity at $T_{c}$ for a fila to be given by the usual equation, which is shown below.

$$
\Delta C=C_{s}-C_{n} \text { and } \Delta C=1.43 C_{n}
$$


Thus, one would expect the heat capact ty in the superconducting state $C_{s}$ near $T_{c}$ to be equal to $2.43 C_{n}$. Th1s means that the rise of the total heat capacity plotted in Figure 21 is between 5.5 and 11 times the expected B.C.S. Jump, depending on what percentege of background contributions are present in the total measurenent. This rise is occurting 18 mo below the MFT transition tenperature (pofint $A$ ), as determined from an $A, L$. fit to the resistive transition. This rise is also very near ( $\sim 2 m k$ ) the tenperature where the film's resistance becomes zero. It is possibly only a cotncidence that the temperature range of the resolution of the experiment $\left(P, P^{\prime}\right)$ is on the order of the temperature difference between point $A$ and point $B$. The excess heat capacity is defined as the difference between the normal and the superconducting heat capacities $\left(c_{\text {excess }}=c_{s}-c_{n}\right.$ ), and should be zero far above $T_{c}$, and equal to $\triangle C$ far below $T_{C}$ on the plot of figure 21. The logarithric plot of the excess heat capacity in the insert of Figure $2 l$ is for the range in temperature indicated by the arrows. This plot indfcates linear relationship between the logarithm of the excess heat capacity and temperature. Since the oscillations in temperature ( $\Delta T$ ) used in this heat capacity measurement are considerably less than the thermal fluctuations of the sample $(\Delta T \sim 1 \mu K, T \Rightarrow T \pm 10 \mu K)$, the measurentent is essentiatly high spead average (averaging time $* 1$ cycle $=10^{-3}$ sec) over a region in temperature $10 \mu K$ wide. In principle, the wathod of correlation in $R$ could resolve this width in temperature, but beckuse long relative tine constants are chosen for the electronics used in the 
measuremant ( $\mathrm{sec}$ ), and since the temperatuce of the $\mathrm{f} f 1 \mathrm{~m}$ is continuadiy increasing in time, the total heat capacity measured will always be an integrated measurement over a broader range in temperature. This is an inherent 1 imit on the ability of the experiment to determine heat capacitfes changing very rapidiy with temperature. As stated earlier, this limft is an average over, at most, 6 neighboring points in temperature. This means that the steeper part of the rise of the heat capacity in figure 21 oceurs over a region that is $1 \mathrm{mK}$ wide at nost. The total rise occurs over a range of about 6 ak. This width wtll be diseussed later.

\section{Electrical conductivity}

As stated in the theoretical section, the conductivity of the films would be studied to determine if their transitions were broadened by Inhomogenefties. This determination comes about by fitting the resistive transition to the theoretical form of A,L. theory, given earlier. The width of the transition for this fit $\tau_{0}$ is compared to the theoretically derived width $\tau_{0}^{A . L}$. Inhomogeneities would tend to broaden the transition and make $\tau_{0}>\tau_{0}^{A . L .}$. There are theoretical models that relate intrinsic brodening for ratios of $\tau_{0} / \tau_{0}^{A . L}>1$ to an intrinsic pair breaking process daterained by the value of $R_{D}^{N}$ This is discussed In Appendix H. Table 31 ists the fitted transition width for the particular films discussed here. Some values for $R_{a}^{N}$ and the ratio $\tau_{0} / \tau_{0}^{A . L}$. are in agreement with the A.L. theory, but in disagreement 
with the theory of Patton, discussed in Appendix $H$. These results are in better agreement with the work of Thompson, ett.af. 43 , except that only fitm $\$ 30$ exhibited the 0 . C. current features they observed. Although some transition widthe agree w1 th A.L. theory, the ftlus are probably not howogentous because of the long tails in the transftion (as compared with the sharp transitions in Gtover's work of amorphous $\mathrm{Bi}^{46}$ ). Thompson, et. ‥ suggest that these tajls in the transitions of dfrty aluminum films are due to the thermally excited phase fluctuations in the coupling between grains of the fflm, destroying long range order and creating a resistance In the f1lm, but still maintaining a non-zero value for the order parameter. Thus, the transition width deternined from conductivity neasurements is not a direct measurement of the width of the superconducting phase transition in aluminum films. The distribution of the transition temperatures of the grains in the film produces a pramature transition at higher temperatures as a result of a few grains "shorting" out the contribution to the resistance from the majority of the grains. At the same time, phase fluctuations between grains will result in a fintte resistance below the superconducting phase transition. Figure 22 shows the resistive transition for film \#39. An A,L. fft with the paramerers show is also plotted. The insert of Figure 22 shows the excellent agreement of the A.L. theory with the resistive transition at higher teliperatures. The parameters are determined by choosing a value for ps that is consistent with the measured values of the f1im. Then the inverse 
RUN $\# 39(\mathrm{H}=0.0 \mathrm{O})$

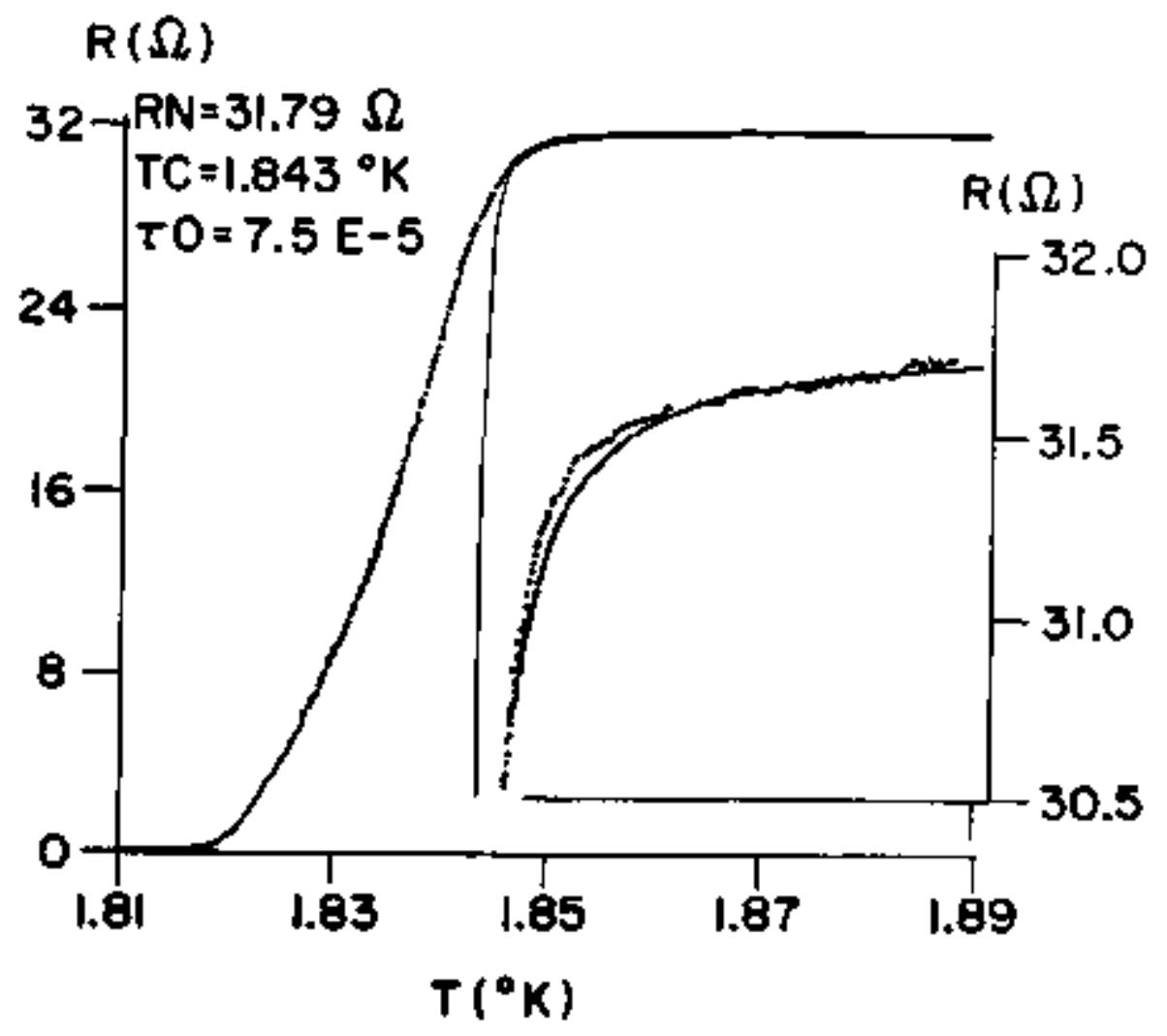

Fig̨ure 22.

Reststive transition for $f \mathrm{~lm}_{\mathrm{m}} \mathrm{A39}$. The plot is of 1020 discrete points. The A.L. fitted parameters are shown, along with the fit. WW is approximated and 15 very close to the $R H$ (for $T \gg T_{c}$; measured. $T_{c}$, $T_{0}$ are determined graphically (see Figure 23). The insert shows the good agreement with the fit at the high temper ture end plotted on the same temperature scale. 
of the excess conductivity (where $\frac{\sigma^{\prime}}{\sigma_{N}}=(R+-R) / R$ ) is plotted in Figure 23, and a linear fit is used to determine the transition temperature $T_{c}$ and the transition width $\tau 0$. This type of fitting can give varied results, depending on the region over the data which the theory is forced to fit. The parameters $T_{c}$, PH, and ${ }^{\circ} \theta$ can be varied over considerable ranges that result in ffts over many separate regions of the transition. Obviously the theory cannot fit the whole transition, so that the choice of where the resistance of a filen fits to the theory is extremely arbitrary. Roughly, the parameters vary the theoretical function for the film's resistance in the foltowing way. The width parameter to determines the angle between the steep region of the transition and the flat region. The value for the normal resistance RN deterwines the value of the resistance and the slope at the high temperature part of the transition. And finally $T_{c}$ moves the overall features up and down in texperature. These fumetional variations are not independent, but by the use of the Tektronix display mentioned in figure 19 and. used in all the plots of this section, one can see this rough variation of the theoretical transition by varying the parameters $T_{c}$, RN, to. In this way, the transition can be fit to the A.L. theory in any arbittrary region. It is quite possible that the premature transition brought about by the "shorting" of a few grafns in the film is creating the slope at the high temperature end of the transition, which results in the high $T_{c}$ obtained in the A.L. fit. It is more likely that the real transition is occuring 


\section{RUN $* 39(H=0$ 0.)}

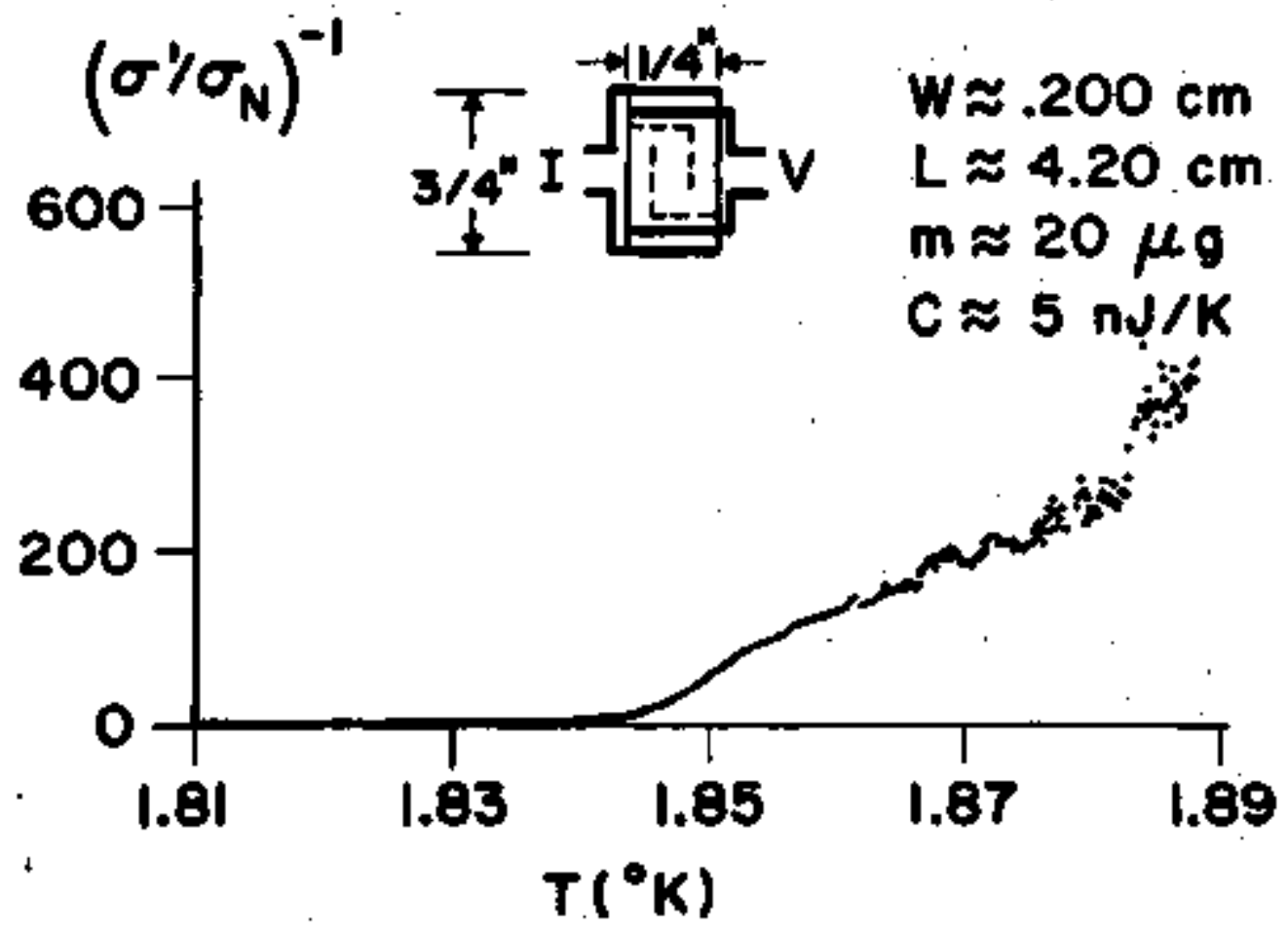

Flgure 23.

Inverse of the excess conductivity ratio plotted vs, temperature for Run 139. The volue chosen for RN is that used in Figure 22. A linear form is fitted to the data to obtain the $T_{f}$ and $\tau_{0}$ used in the fitted plot of Figure 22. The insert shows the film's geonetry with typical parameters. The films are edged scribed and also scribed with the pattern scribe shown (to increase the film's res 1stance). 
in a region where the majority of the grains are going superconducting, and would be at a temperature considerably lower than the fitted value for $T_{\mathfrak{c}}$ used in Figure 23. Thus, it is believed that the real superconducting phase transition in dirty aluminum fi) actually be occuring in the region where $R \approx 0$. This idea is supported by work done in this lab which measured the excess tunneling current in $5 n-\mathrm{Pb}$ Josephson junctions for $T>T_{c}$ of the $\mathrm{Sn}^{45}$. This work indicated that the phase transition, as determined by the excess tumneling current, was actually occuring near $R=0$ for the $\mathrm{Sn}$ film. Thus, although the conductivity of the films discussed in this section is in agreement with the A.L. theory, it is quite possible that their transitions are broadened due to the inhomogeneities brought about by the different transition temperatures of the various grains in the film. This model could possibly explain why the transition widths of the heat capacity results of Figure 21

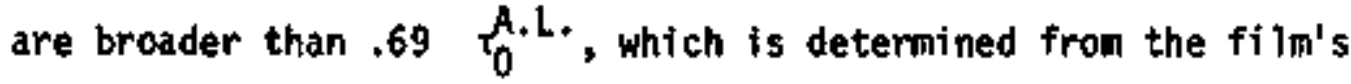
conductivity; and it could explain why the rise in the heat capacity of the film is occuring at a temperature far below the $T_{c}^{M F T}$ determined from the A.L. fit.

\section{Heat Capacity}

The general features of the total heat capactty plotted in Figure 21 were observed in 9 films. One of these films was a fully self-supported film (see Appendix H) that had a measured relatfve heat capacity on the order of that observed for the 
filus on mica substrates. This indicates that the total heat capacity measured for the films on mica might be a $100 \%$ measurement of the heat capacity of the film itself.

figure 24 shows the tota 1 heat capacity measured for fitm $\$ 30$, plotted for three ranges of temperature. The heat capacity exhibits rise from a constant value at the low temperature end of the transition. The higher resolution in temperature shown in Figure $24 \mathrm{c}$ indicates a peaking of the total heat capacity. Using the same estimates as given earlier in Figure 27 , this results in a rise of between 4.5 and 8.6 times the expected B.C.S. jump in heat. capacity. The temperature width in this rising region is about $5 \mathrm{mK}$ wide. which is considerably larger than the $\tau_{0}^{A}$. L. width parameter from A.L. theory. But $1 t$ is within a factor of 10 of the value for $\tau_{0}$ that is used to fit to the resistive transition. Since the ratio of ${ }^{2} 0^{\prime} \tau_{0}^{A . L .}$ is greater than unity $(* 17)$, this might be an intrinsically broadened film, although it is still in disagreement wf th the theory of Patton, discussed earlier (i.e., the ratio is too large for $t$ ts value of $\left.R_{a}^{N}\right)$. Another possibility for the observed peaking in Figure 24 may be a result of its granular nature. Since this film exhibits a strong dependence on D.C. current, and since it was prepared in a heavy oxidizing atmosphere, the Josephson tunneling between grains could cause large order parameter phase fluctuations between the grains. This peaking in the heat capacity may be related to a transition from " "paracaherent" coupling of the grains to a coherent coupting brought about by the electron tunneling, and thus the phase transition 
RUN 30 I

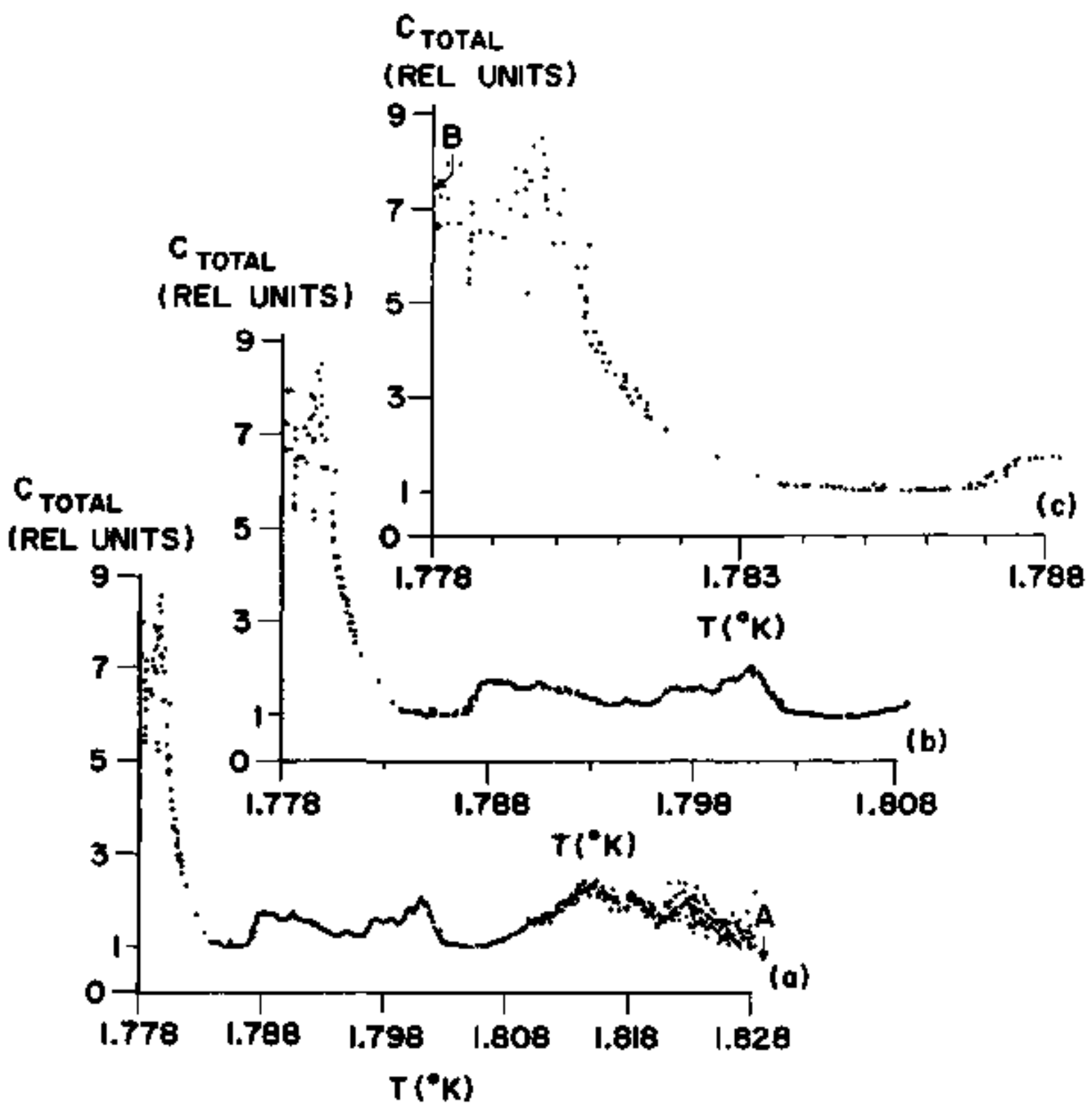

Figure 24.

Total heat capacity plotted for Run $\$ 30 I$. The temperature scales have been enlarged to show the rising heat capacity near point $B$. a) is $50 \mathrm{mK}$ wide, b) is $30 \mathrm{mik}$ wide, and c) is $10 \mathrm{mK}$ wide. There is an apparent peaking and then a-leveling off in the date near point $B$. 
in the heat capacity may be a consequence of the ordering of the grains. This phase transition is discussed in Ref. 47 with regard to an interpretation of conductivity measurements made on bulk powdered $\mathrm{Nb}$ and $\mathrm{Ta}$ samples in which a precipitous drop in the resistive transition is observed at a low temperature tail.

Flgure 25 shows the resistive transition of fi3a $\$ 36$. This film has a multiple transition, with the first transition at about 1.88K. Figure 26 shows the total heat capacity for film $\$ 36$ with the resistive transition over the sakle temperature range superimposed on the plot. There is a definite peaking of the heat capacity over a region of $15 \mathrm{mk}$ which $r$ ises to a value of between 12 and 25 times the B.C.S. jump, at a temperature of about $12 \mathrm{mK}$ below the $T_{C}^{M}$ (point A). But there is also rise in the heat capacity of a similar neture at the low temperature region as seen in the previously mentioned flims. This rise occurs over about a 5mk region and increases to a value of between 4 and 7.6 of the B.C.S. juap. Oue to the multiple transition in the film, the thernometer in effect has a range that is extended below the transition of the first film, and thus allows the measurement of heat capacity below the first transition. The results of figure 26 indicate a possible peaking in the heat capacity of the $f 1 \mathrm{Im}$ at the first transition, and a consistent rise near $R=0$.

\section{Magnetic Field Effects}

Because of the predicted effects of the alteration of the heat capacity by the application of a small magnetic field, 


\section{RUN *36B}

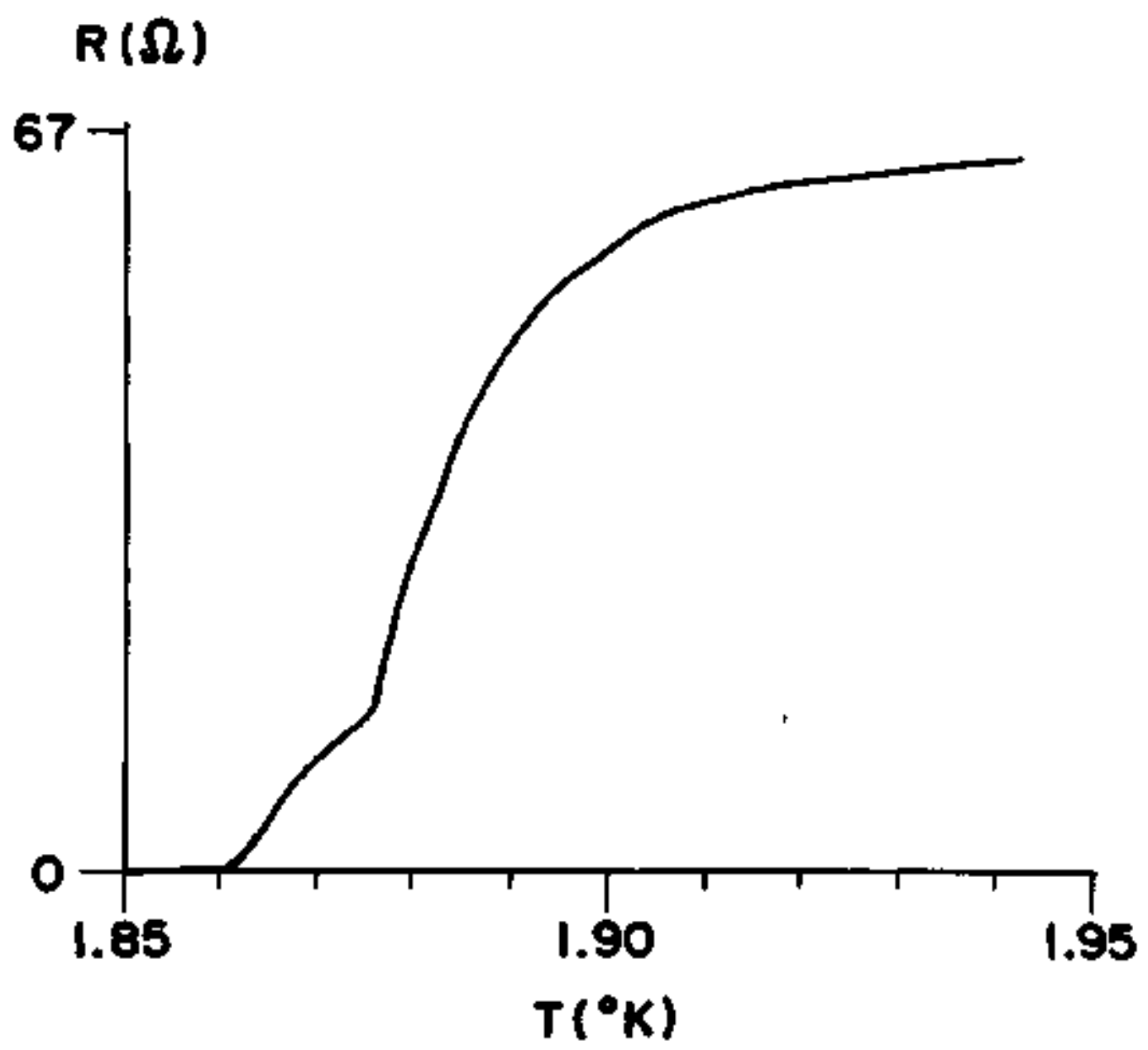

Figure 25.

Resistive transition for Run $136 \mathrm{~B}$ showing the multiple transition. 
RUN $36 \mathrm{~B}$

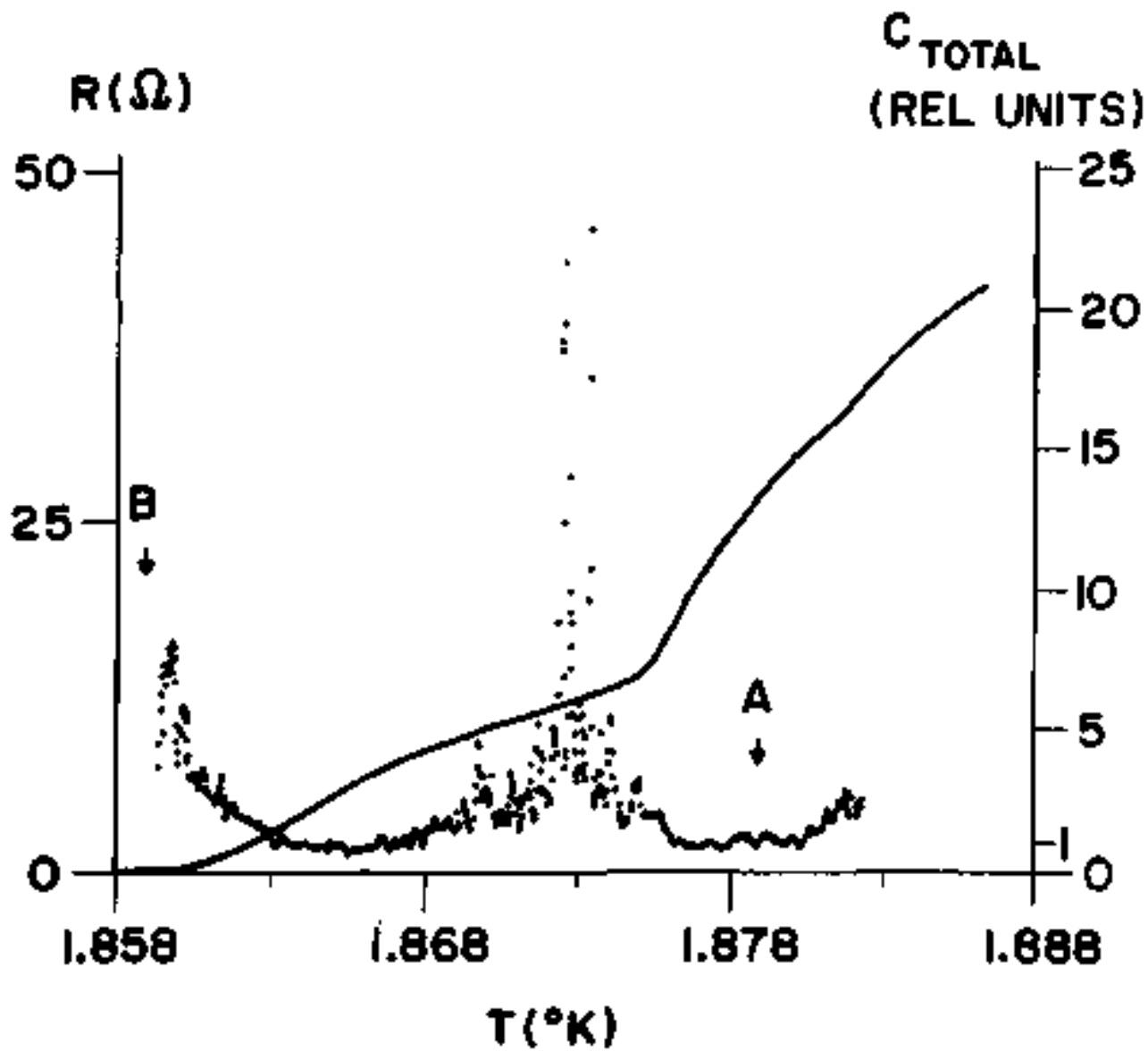

Figure 26.

Resistive transition and heat capacity for Run $\$ 368$. The resistance plot is of the tall of the transition shown in Figure 25 . The heat capacity exhibits peaking at a temperature below the 1st resistive transition and a slow rise at the low temperature end. 
discussed in an earlier section, a run was made with a rather crude solenoid attached to the cryostat. This allowed for the application of noderate magnetic fields (although they were about 10\% inhomogentous), which is discussed in more detaji in Appendix $K$. The heat capacity shown in Figure 27 is for run $\# 39$ and it is without an applied field. The excess heat capacity is plotted vs. temperature, and it is missing a section of data, due to the inaccurate cholce of the background subtraction. This is reflected in the low values in the same temperature region on the logarithmic plot of the excess heat capacity of Figure $27 \mathrm{~b}$. Hote the rather large peaking in the heat capacity at the low temperature end (16+32 BCS) near point $B$. The rise occurs over a range in temperature of $10 \mathrm{mK}$ with the peak being on the order of $3 \mathrm{mk}$ wide. The peaking occurs at a point about $25 \mathrm{mK}$ below the THFT determined from the fit of Figure 22. The logarithmic plot varies linearly with temperature in the rising heat capacity region, in a maner simflar to that of Figure 2t. This might indicate a divergence, where the predicted peaking from the screening approximation discussed in an earlier section is just the first term of a serles in powers of $\varepsilon^{-1}$ (see Ref. 41). The inverse of the excess heat capacity is plotted in Figure 28. This type of a plot suffers from the inaccurate determination of the background heat capacity $C_{\text {background }}$ that is similar to the problem of the chofce of RN used in the plot of Figure 23. The application of 21.8 De magnetic field caused a change in the resistive transition, mentioned in Table 3 and shown in Figure H-S of Appendix H. The major feature was to shift the transition to a lower temperature (point $A$ is lowered in temperature) 


\section{RUN *39}

LOG C EXCESS

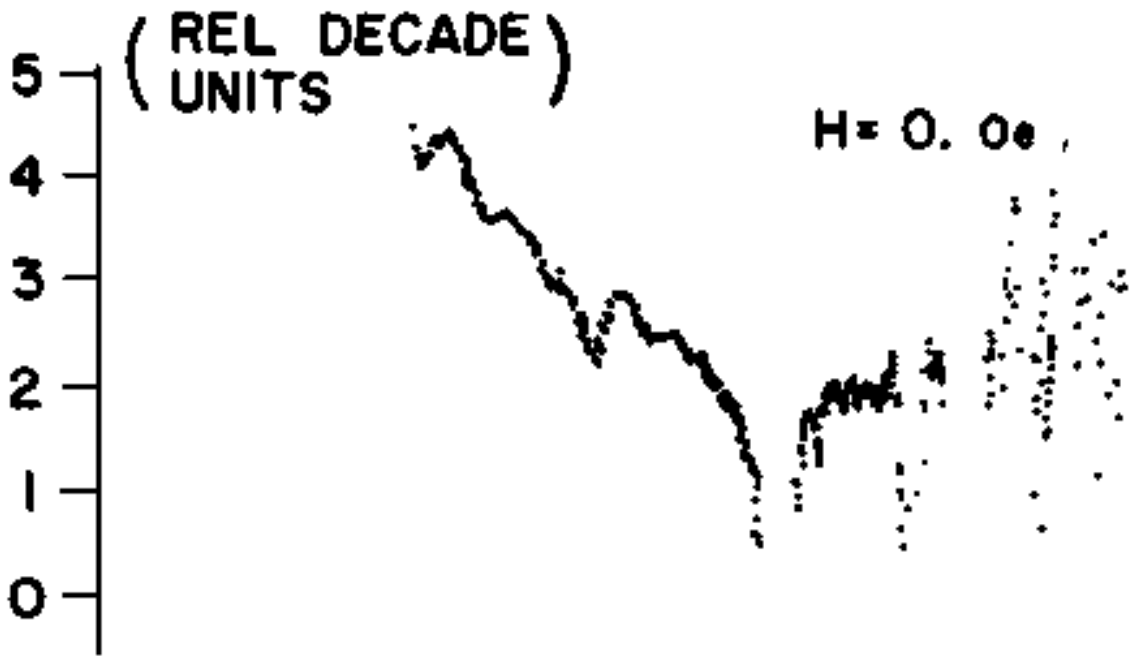

(b)

$C_{\text {EXCESS }}$

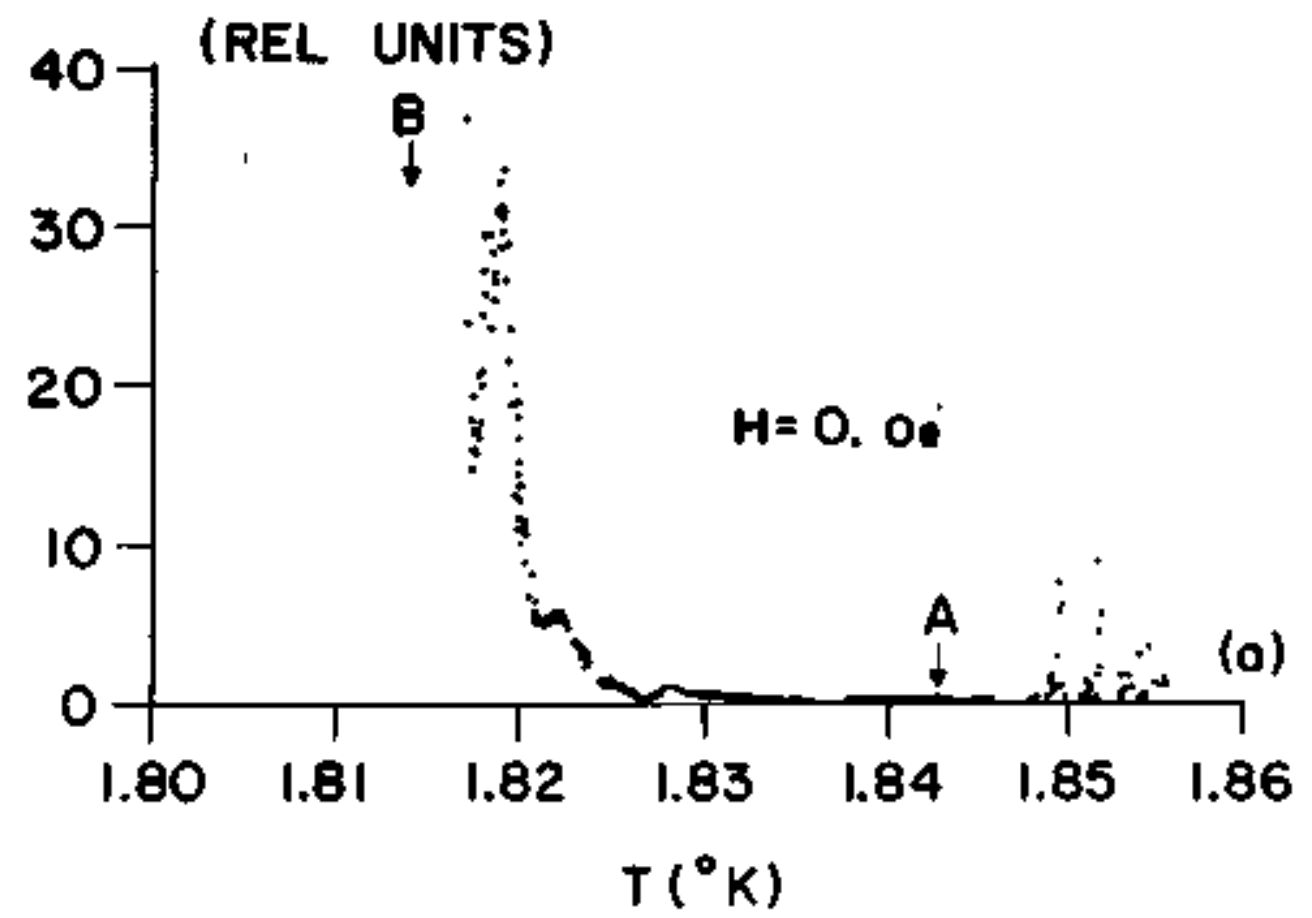

Figure 27.

a) Excess heat capacity plotted for Run $\# 39$. The $C_{\text {background }}$ little too large at about $1.835 \mathrm{~K}$, and results in a negative (not shown) value for the excess heat capacity. This is reflected in the dip of the logarithmic plot of $b$ ). The rising region of a) has a linear form on the logarithmic plot of b) and exhibits a defintte peaking. 
RUN *39

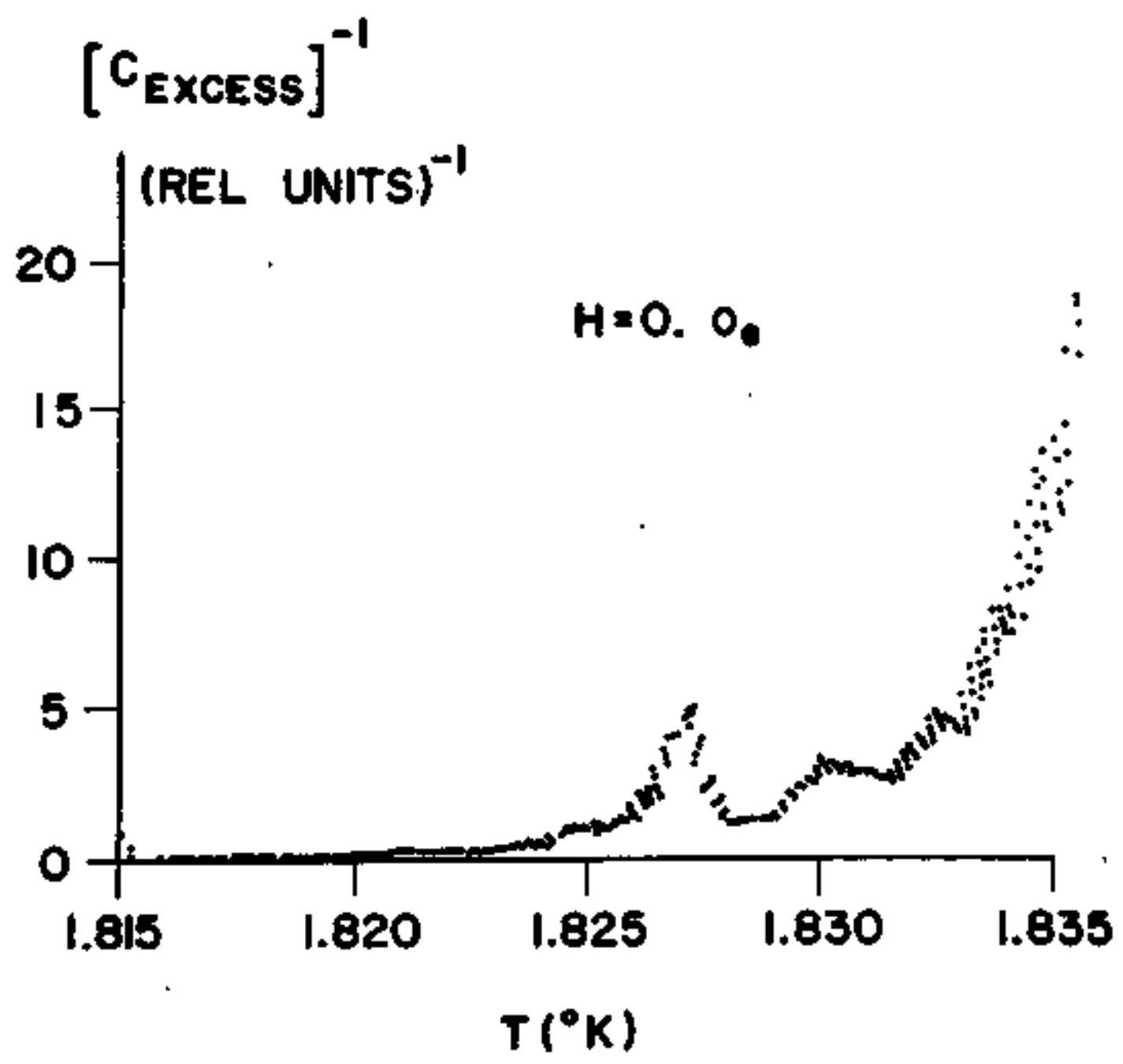

Figure 28.

Inverse of Excess Heat Capactly for data of Figure 27. The plot is over temperature range where the background subtraction is not important. The determination of the subtraction constant is the same problem as the determination of RN used in Figure 23. Note the similarity in the low valued linear regions of the plot of Figure 23 and the plot shown here. 
and to provide a tail in the transition that extended the thermometry (the difference between $T$ (pt.B) and T(pt.A) is increased). The total heat capacity measured in a magnetic field is shown in Figure $29 b$ with the zero magnetic field result also plotted over the same width in temperature. The dramatic result shown in Figure 29 is that the peaking of the heat capacity in zero magnetic field is reduced, broadened and shifted to a lower point in temperature when a magnetic field is applied. The shift is to a point $43 \mathrm{mK}$ below the zero magnetic field result, and over a widened temperature range, wich is on the order of $30 \mathrm{mk}$. The extended thermometry due to the tall in the resistive transition brought about by the appiled field is probably related to magnetically induced phase slippage in the coherence of the Josephson coupling between grains. This extension allows for the deternination of a plateau in the heat capacity of the $f 11 \mathrm{~m}$ in an appiled field, at a temperature below the rise seen in the heat capacity of films discussed earlier. One should notice the reproducable bumps in both Figures $29 \mathrm{a}$ and $29 \mathrm{~b}$ in the region of rising heat capacity. The magnetic field results are in qualitative agreement with the fluctuation predictions presented in the theoretical section.

\section{Ditcussion}

The results presented earlier indicate a rather large rise in the heat capacity of the four $f i i_{\mathrm{ms}}$ discussed at a temperature below the $\mathrm{T}_{c}^{\mathrm{MFT}}$ (determined from conductivity measurements). The 


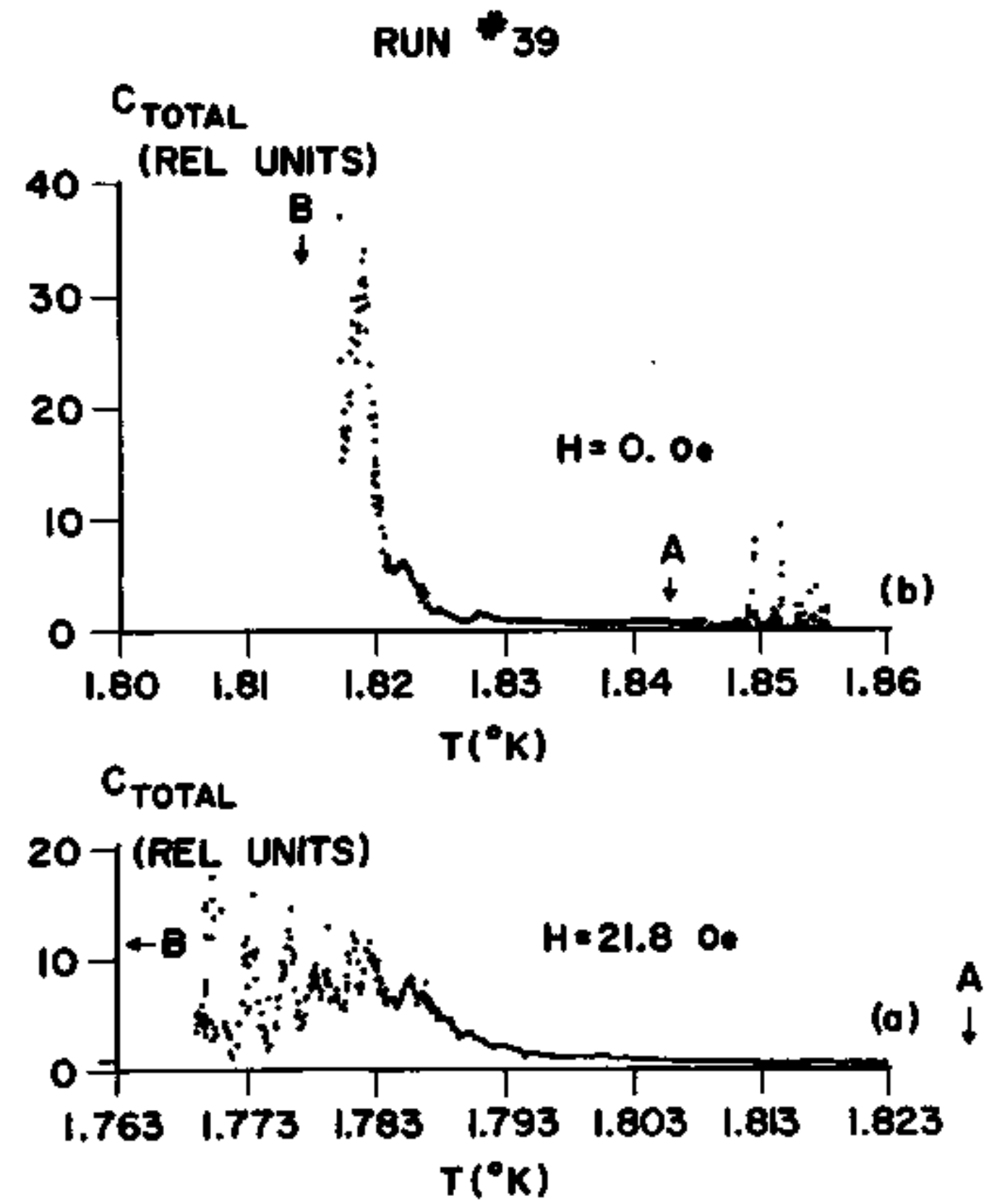

Figure 29 .

Total heat capacity of rum $\$ 39$ plotted for two values of magnetic field on the same relative temperature range. a) is for a field of $21.8 \mathrm{oe} \quad$ b) is for no magnetic field. The magnetfe field shifts the transition to a lower temperature and broadens the region where the heat capacity is peaking. At the same time tt reduces the size of this peaking of the heat capacity observed in zero magnetic field. 
posstbility of an error in the analysis of the data should be discussed because of the rather important nature of the results.

The possibility of pair breaking occurting in the film from the Iaser optical heating is discussed in Apperidix $F$ and is believed not to be involved in the present experiment, because the relative number of paired electrons is small at the temperatures of the films studied. Also the pair recombination time is so fast, relative to the heating frequencies, that any disturbed pairs will be recombined just after the end of each heating cycle. The most apparent result was that the resistive transition was unaffected with the laser on or off. This indicates that pair breaking ... mechanisnis due to the optical heating can be ruled out.

A second possibility is that the rise in the heat capacity is associated with a latent heat brought about a local magnetic field. The residual fields $\left(<10^{-4}\right.$ gauss $)$ and the induced fields from the measuring currents $\left(<10^{-5}\right.$ gauss) are far below the fields needed for such effects.

To be consistent with the bulk superconducting phase transition, the entropy associated with a rising heat capacity has to be less than the observed agreements mentioned earlier between the total heat capacity of the superconducting and the normal states. Cochran's result for $S n$ ( $S_{\text {singularity }}<10^{-3} \mathrm{~J} / \mathrm{mole} \mathrm{K}$ ) indicates roughly an entropy for these films of about $10^{-10} \mathrm{~J} / \mathrm{K}$ associated with any hest capacity singularity. The entropy for the observed peak in the heat capacity is just its width $\Delta T P$, times the heat capacity 
peak $C_{p}$, divided by the temperature at which the peak occurs $T_{p}$. The resultant entropy associated with the transition is about $10^{-10} \mathrm{~J} / \mathrm{K}$ (roughty $\mathrm{C}_{p} \sim 10 \mathrm{C}_{\mathrm{S}} \sim 10^{-7} \mathrm{~J} / \mathrm{K}, \Delta \mathrm{T}_{\mathrm{p}} \sim 10^{-3} \mathrm{~K}, \mathrm{~T}_{\mathrm{p}} \sim 2 \mathrm{~K}$ ). As discussed earller. King et. al. found agreement to about $5 \%$ for the entropy difference between the normal and superconducting states. This means that the entropy associated with a phase transition $\Delta S_{Q}$ has to be less than $5 \%$ of the total entropy $S_{T}$. Since $S_{T}$ is just equal to the normal state heat capacity at $T_{c}$, the requirement of $\Delta S_{p}<5 \% \quad S_{T}$ is just the requirement of

$$
\frac{c_{p} \Delta T_{p}}{T_{p}}<5 \% c_{n} .
$$

This reduces to

$$
\frac{c_{p}}{C_{S}} \frac{\Delta T_{p}}{T_{p}} c_{S}<\frac{5 \times 10^{-2}}{2.43} c_{5} .
$$

For the largest peak observed $\left(C_{p} / C_{S} \sim 20, \frac{\Delta T_{p}}{T_{p}} \sim 10^{-3}\right)$ this condition is satisfied.

It should be noted that the prediction of no phase transition in 2-0 systen, mentioned in the theory section, relies on the fact that the order parameter correlation function of the 2-D superconductor does not exhibit off diagonal long range order (OOLRO). It actually states that in 3-0, there is ODOOR, and hence one can have a divergence in the heat capacity. But in 2-D, where one does not have ODLRO, one may or may not have a divergence.

The fact that the data is analyzed by an equivalent circuit may also be reason for the observed heat capacity. But the 
equivalent circuit used is understood very well, and nine films, all with very different sets of raw data and different resistive transitions, had similar overall features.

The disagreement between the peak width $\Delta T_{p}$ and the $\tau_{0}^{A . L}$. from the conductivity measurements, and the disagreement between $T_{p}$ and $T_{c}^{M F T}$ is not surprising in view of the discussion of conductfvity measurements. It might be possible to use a Gaussian distribution in $T_{c}$ 's similar to that of Cochran to represent the grain inhomogeneities contributing to the broadening of a narrow intrinsic peak. In this case, the coefficient of the diverging term (a) would be varied to get the correct height of the peak, and the range of integration would be over the width of the peak. This model might also expiain the disagreement in the conductivity and heat capacity transition widths.

Finally, it should be mentioned that in this laboratory, ${ }^{44}$ there has been other observed behavior of a possible critical region in very similar aluminum films. In the measurements of the inverse peak current of $\mathrm{Pb}-\mathrm{A} 1$ Josephson junctions below the $\mathrm{T}_{\mathrm{C}}^{\mathrm{MF} T}$ (13 mK below), there is a region $10 \mathrm{mK}$ wide where the GL M.F.T. breaks down.

\section{Conclusion}

Because of the theoretical interest in phase transitions, the heat capacity of a two dimensional superconductor has been measured in the transition region. Previous experimental work $(\mathrm{ZH})$ was 1 imfted by the arbitrary fitting schemes used in the data 
analysis. The previous work was also limited by the ultimate sensitivity of the experinent. The work reported here used unconventional methods to measure the heat capacity of a $1000 \mathrm{~A}$ granular aluminum fllm with an unprecendented sensitivity. The film was heated optically and the fiTm's resistive transition was used as its own thermometer. The electrical conductivity and heat capacity results for the four films presented were in qualitative agreement with simflar results observed in five other fitms.

The heat capacity measured from the data on the four films presented increased with decreasing temperature where the fllms' resistances were zero. The rise was over a range of 1 to $5 \mathrm{mk}$ and to a height of up to possibly 32 times the B.C.S. discontinuous jump. Two films exhibited a definite peaking of the low temperature region of this rising heat capacity. This peaking occured at a point about $20 \mathrm{mK}$ below the MFT transition temperature determined from the conductivity measurements. It is believed that the granular nature of the films broadens the resistive transition to give an arbitrarily wider transition, such that the temperature associated with the onset of order over most of the sample is not in agreenent with the $T_{c}^{M F T}$ determined from a fit to the resistive transition. The phase transition is most likely occuring in the region where the $\mathrm{ftIm}^{\prime} \mathrm{s}$ resistance is going to zero. An applied magnetic field caused the observed peak of one film to be reduced in height, broadened, and shifted to a lower temperature.

The observed peaking of the heat capacity of these granular aluminum films may be an actual broadening of a much narrower 
intrinsic peak due to the distribution in grain size. It might. also be the result of a phase transition associated with the coupling between grains due to pair tunneling producing a transtition from a "para-coherent" to a coherent state.

These results are in partial agreement with the predicted non-monotonic behavior, but the peak in the heat capacity is much larger than the results of the most recent calculations. 15 However, these calculations are just the first order corrections to the Hartree approximation and the inciusion of higher order terms may cause the heat capacity to diverge.

The measurements were 1 imited to the transition region because of the limited sensftivity in using the film's resistive transition for thermometer. The requirements of thermal equilibrium in measurements of the heat capacity and the very low mass of the samples make an intrinsic measurement of the film's temperature essential. In this work, an intrinsic measurement was obtained using resfstive themonetry. It might also be possible to couple a small tumneling junction to the film, with the film being one of the superconducting electrodes. With this arrangement, one could use a feature of the temperature dependent tunneling characteristic (such as the pair field susceptibility metnioned earlier) to extend the measurements outside the transition region. This would then enable the intrinsic temperature dependence of the heat capacity of the film to be determined outside the transition region. In conclusion, the heat capacity of a thin aluainum film has been measured in the transition region. A peak has been observed 
in a region where the film's resistance is beconing zero. This peak is in partial agreement with predictions, but it indicates a qualitatively larger value than that predicted. This implies that the predictions are only a first order correction to the Hartree approximation and that higher order corrections have to be considered. 
List of References

1. H. E. Stantey, Intro, to Phase Transitions and Crittcal

Phenomena, Oxford University Press, K. Y. 1971.

2. L. P. Kadanoff, et.a1., Rev. Mod. Phys., 39, 395 (1967).

3. D. ter Haar, Men of Physics: L. D. Landau, 2, Pergamon Press, New York, 1969. See pg. 61 which is a translation of the original paper appearing in Phys". Z. Soviet Un. 11, 26 (1937).

4. J. Bardeen, L. N. Cooper, J. R. Schrieffer, Phys, Rev, 108, 1175 (1967). A comparioon can be made from the presentations in Superconductivity of Metals and Alloys by P. 6. De Gennes, W. A. Bendamin, New York (1966). A review artície showing the explicit relationship is given by M. Cyrot, Rep. Prog. Phys, 36, 103 (1973). It was the work of Gor'kov (Sov. Phys. UETP 9 , 1364 (1959) that showed that G1nzburg-Landau theory is Just a limiting case of B.C.S. near $T_{c}\left(i . e ., \Delta=V\left\langle\psi_{+} \psi_{t}\right\rangle\right.$, the pair potential).

5. H. Schmidt, Z. Phystk 216, 336 (1968).

6. R. A. Ferre11, J. Low Temp. Phys. 1, 241 (1969).

7. W. E. Masker, S. Marcělja, R. D. Parks, Phys. Rev, 188,745 (1969).

8. P. A. Lee, S. R. Shenoy, Phys. Rev. Letters 28, 1025 (1972).

9. S. Grossman, P. H. Richter, C. WisseT, Solid State Comm. II. 433 (1972).

10. W. E. Masker, S. Marcelija, R. D. Parks, Phys, Rev, 188, 745 (1969).

11. Shang-keng Ma, "The Renomal, Group and the Large $n$ Limft", preprint, U of Calif.. San Diego, La Jolla, Calif., 92037. It should appoar in the J. Math Phys. about August 1974. 
12. L. W. Gruenberg, L. Gunther, Phys. Letters 38A, 463 (1972\}.

13. L. Gunther, L. W. Gruenberg, Solfd State Comm. 10, 567 (1972).

14. A. J. Bray, G. Rickayzen, J. Phys. F: Metal Phys. 2, L109 (1972).

15. D. J. Scalapino, R. A. Ferrell, A. J. Bray, Phys. Rev, Letters 31, 292 (1973). The "Screening" approximation itself is discussed in the paper by R. A. Ferre11, D. J. Scalapino, Phys. Rev, Letters 29. 413 (1972).

16. D. J. Thouless, Ann. Phys. 10, 553 (1960).

17. A. P. Levanyuk, Sov. Phys. - Solld State $\underline{5}, 1294$ (1964).

18. W. Weller, Phys. Letters 5, 35 (1965).

19. T. Tsuzuki, Progr. Theoret. Phys, 41, 296 (1969).

20. L. P. Kadanoff, G. Laramore, Phys. Rev. $\underline{175}, 579$ (1968).

21. H. Schnidt, "Fluctuation in Superconductors Above $T_{c}$ ", Int. Low Temp. Conf, $\$ 11, \mathrm{pg} .798$ (1968).

22. H, Schratdt, Z. Phys1k 232, 443 (1970).

23. L. 6. As 1 andzov, A. T. Larkin, Phys, Letters 26A, 238 (1968).

24. L. 6. Aslamazov, A. T. Larkin, Sov. Phys. - Solid State 10, 875 (1968).

25. K. Mak1, Progr. Theoret. Phys. 40, 193 (1968).

26. J. B. Parkinson, J. Phys. F: Metal. Phys, 2, 966 (1972).

27. R. F. Hassing, R. R, Hake, L. U. Barnes, Phys. Rev, Letters 30, $6(1973)$.

28. R. F. Hassing, J. W. Wilkins, Phys. Rev. B7, 1890 (1973).

29. S. Grossuann, P. H. R1chter, Physics Letters 33A, 39 (1970).

30. B. R. Patton, Phys. Rev, Letters 27, 1273 (1971). 
31. S. Marcélfa, Phys, Letters 35A, 335 (1971).

32. W. E. Masker, 5. Marcélja, R, D. Parks, Phys. Rev. 188,745 (1969).

33. R. A. Craven, G. A. Thomes, R. O. Parks, Phys, Rev. B7, 157 (1973).

34. J. F. Cochran, Anns. Phys. 19, 186 (1962).

35. L. J. Barnes, R. R. Hake, Phys, Rev. 153, 435 (1967).

36. R. F. Hassing, R. R. Hake, L. J. Barnes, Phys. Rev. Letters 30, 6 (1973).

37. G. D. Zally, J. M. Moche1, Phys, Rev, Letters 27, 1710 (1971).

38. G. D. Zally, J. M. Moche1, Phys, Rev, 86, 4142 (1972).

39. R, L. Greene, C. N. King, R, B. Zubeck, Phys. Rev. B6, 3297 (1972).

40. W. Buckel, Ch, Ohlerich, "Spectfic Heat of Strongly Disordered Crystalline and Amophous Fflms", Int. Low Temp. Conf. \$13, Boulder, Colorado, August 1972.

41. G. Rickayzen, A, J. Bray, "Fluctuat fons in Superconductors: The Screening Approximation in Two Dfmensions", to be published in J. Phys. F: Metal Phys. $\underline{3}$ (August 1973).

42. Note that this assumes that $\Delta V$ is a constant voltage source. If the problem is formulated with a constant current source and continutity of currents is used $\left(I_{D C}-I_{R}+I_{Z}\right)$, the results differ only by a constant which is posstbly unity.

43. R. S. Thompson, M. Strongin, O. F. Kamnerer, J. E. Crow, Phys ics Letters, 29A, 194 (1969).

44. R. V. Carisan, A. M. Gotaman, "Superconducting Order Parameter Fluctuations Below $T_{c}$ ", submitted for publication to the Physical Review Letters (August 1973). 
94:

45. J. T. Anderson, indirect private communication.

46. R. E. Glover, Physics Letters 25A, 542 (1967).

47. P. Pella n, G. Dousselin, H, Cortes, J. Rosenblatt, Solid State Corm. 11, 427 (1972).

48. Measurements were made without a transformer with large temperature excursions $(\sim .1 \mathrm{mk})$ that verified the results obtained with a transformer and using this equivalent circuit. 
Appendix A. A.C. Bridges and Thermonetry.

The thermometry for the experiment involved accurate measurement of the resistance of both cartion and Germantim resistors, the later being comerically available and callibrated by $a \log R$ (resistance), $\log T$ (teaperature), extrapolation between points tracesble to the M.B.S. (absolute accuracy $\sim 1$ mik).

The A,C. Resistance Bridge (Figure A-1) is a variation of the bridge used previously in our Taboratory. ${ }^{2}$ The bridge is referenced with a woltage from a lock-in amplifier (P.A.R. - HAR-B) and the signal from the null output is phase sensitive detected by the lock-in ampilffer until both the ratio box and capacitance box settings give a null voltage on the lock-in output. The equation for the voltage output of the HR-8 is:

$$
\begin{aligned}
& v_{\text {out }}^{H R-8}=\frac{1}{\tau} \int_{0}^{\tau} \text { dt } I_{R}\left\{R_{s}\left[1+R_{s}^{2}\left(\frac{L^{\prime}-C^{\prime}}{\omega L^{\prime} C^{\top}}\right)^{2}\right]^{-1 / 2}\right\} e^{i \phi_{s}} \\
& x \in \propto\left\{1-e^{i\left(\phi_{x}-\phi_{s}\right)}\left[\frac{R_{x}\left[1+R_{x}^{2}\left(\frac{L-C}{\omega L C}\right)^{2}\right]^{-1 / 2}}{\left.\alpha R_{s}\left[1+R_{s}^{2}\left(\frac{L^{\prime}-C^{\prime}}{\omega L^{\prime} C^{\top}}\right)^{2}\right]-172\right]}\right\} e^{-1 \Delta \phi_{r}}\right.
\end{aligned}
$$

where

$$
\begin{aligned}
& \omega \sim 2.4 \mathrm{KHz} \alpha=\text { bridge factor } \\
& \text { including ratfo setting } \\
& \phi_{s}=\tan ^{-1}\left[\frac{\alpha R_{s}\left(L^{\prime}-C^{\prime}\right)}{\omega L^{\prime} C^{\prime}}\right] \phi_{x}=\tan ^{-1}\left[\frac{R_{x}(L-C)}{\omega L C}\right]
\end{aligned}
$$




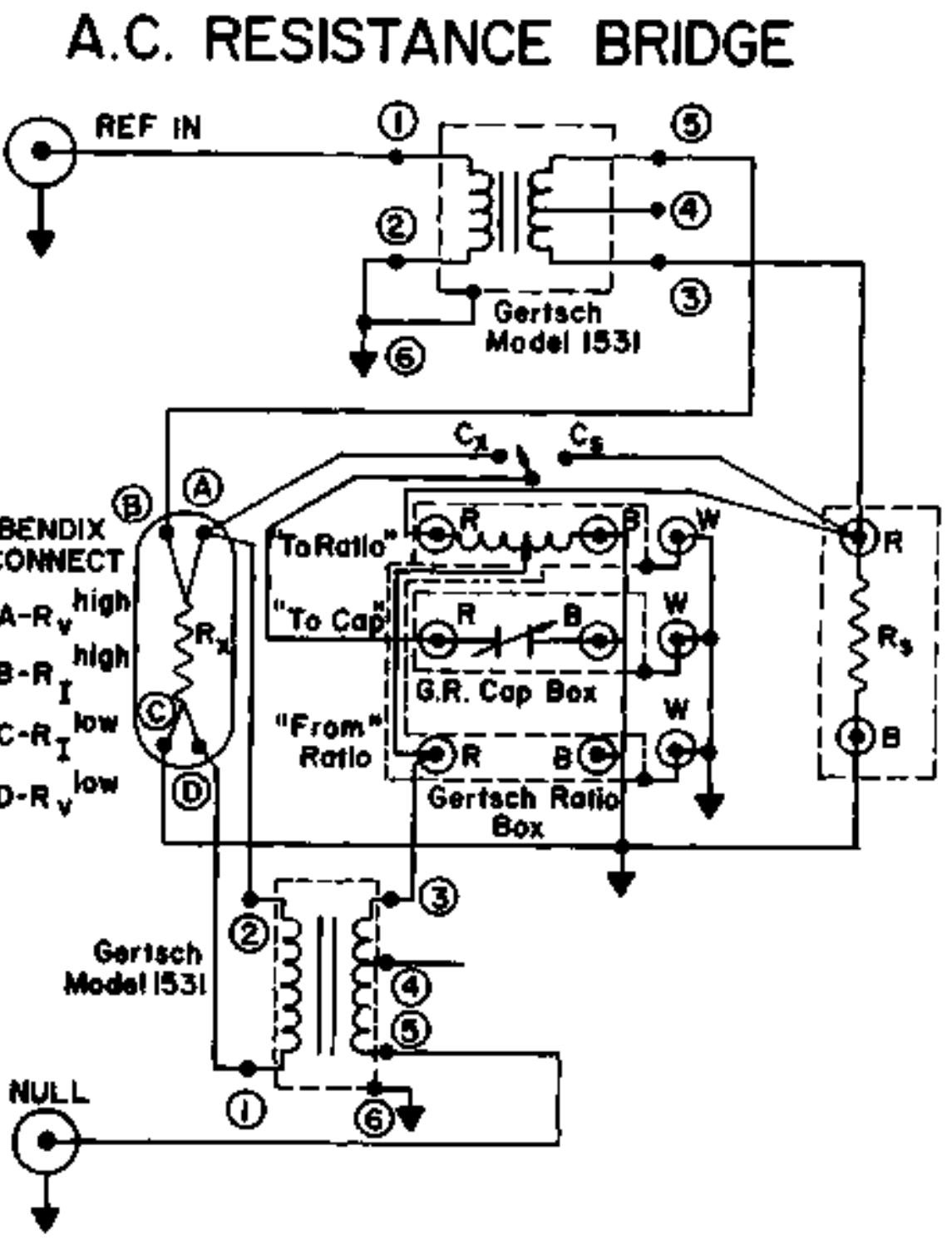

Figure A-1. 
(continued)

$L, C$ are inductance and cap. impedances on the $R_{x}$ (unknown resistor) side;

$L^{\prime}$ and $C^{\prime}$ are inductance and cap. 1mpedances on the $R_{5}$ (standard resistor) side;

$G, \tau, I_{R}$ are gain, time constant, and reference current for HR-8;

$\Delta \phi_{r}=d i f f e r e n c e$ in reference current phase and mixer signal phase which 1 set on the HR-8.

The bridge is balanced by setting $c_{x}$, or $c_{5}$ to zero, and the ratio setting is set to zero. The lock-in output is maximized with the phase setting $\Delta \phi_{r}$ for low sensitivity settings (6-gain). The ratio is changed until a nutl reading is read. The phase is changed by $90^{\circ}\left(\Delta \phi_{r}+\Delta \phi_{r}+90^{\circ}\right)$ and the cap. setting is changed until a null is read. The time constant $(\tau)$ is set at some appropriate value ( $\sim$ lsec). The above procedure is continued for higher sensitivities, until the thermal fluctuations of $R_{X}$ enter in and inhibit more sensitive nulls. For $R_{X} \sim 10 \mathrm{~K}$, $I_{R} \sim 10^{-6}$ anp, these are about $10 \mathrm{nv}$, or $10 \mathrm{\mu} \mathrm{K}$ fluctuations. At this state equation ( $A-1)$ reduces to:

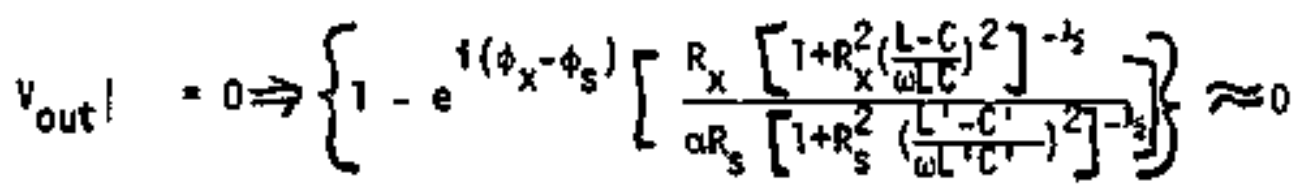

$$
\begin{aligned}
& \alpha R_{s} \sim R_{0}
\end{aligned}
$$

$\Delta \phi_{\mathrm{r}}=0 \quad$ since the coeff. is non-zero 


$$
\begin{aligned}
& V_{\text {out }}+0 \\
& \left(L^{\prime}-C^{\prime}\right) / L^{\prime} C^{\prime} \times(L-C) / L C \\
& \text { combine with }(\mathrm{A}-2)+\phi_{5} \sim \phi_{x} \\
& \Delta \phi_{r}=90^{\circ} \\
& \alpha R_{s} \approx R_{x} \frac{\left[1+R_{x}^{2}\left(\frac{1-C}{\omega L C}\right)^{2}\right]^{-k_{s}}}{\left[1+R_{s}^{2}\left(\frac{L^{\prime}-C^{\prime}}{\omega L^{\prime} C^{\prime}}\right)^{2}\right]^{-\frac{1}{2}}}
\end{aligned}
$$

If one chooses the standard near to $R_{x}$ then to second order:

$$
R_{x} \approx R_{s}
$$

$$
\begin{aligned}
& \text { with a known "unknown" one ean } \\
& \text { measure the bridge factor wi th } \\
& \text { the ratio setting and thus } \\
& \text { measure unknowns, just by knowing } \\
& \text { the ratio setting. }
\end{aligned}
$$

It should be noted that the bridge factor is dependent on the value of $R_{x}$, and thus all experimental values are corrected with this in mind. Also, if one is close to null, and $V_{\text {out }} 0$, one can approximate equation $(A-1)$ by:

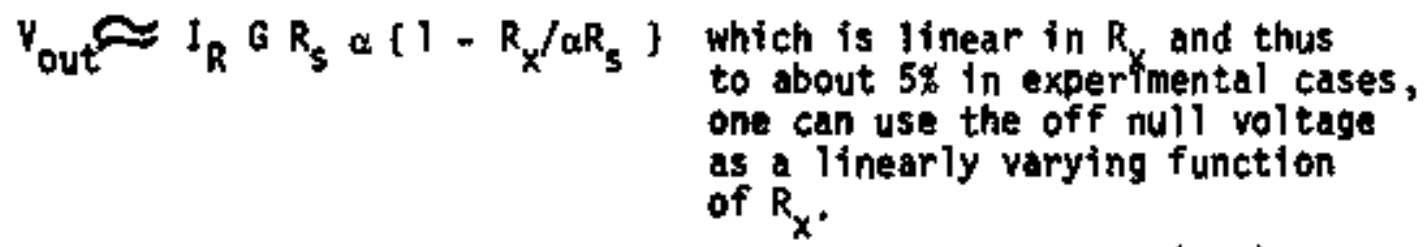

By setting the bridge balance to the center of the varying value of $R_{x}$, and knowing the starting value of $R_{x}$, and the final value of $R_{x}$, one can approxtmately continuousiy monitor $R_{x}$, by mont toring the off null changing voltage (subtracting off the D.C. base Tine) out 
of the HR-8. Th1s method is used not only to obtafn a continuous temperature reading, ffor example taking the resistive transitions as a function of the germanium resfstance), but to use the voltage as a temperature error in an A.C. servo loop (as will be discussed Tater).

The Self-Balancing Bridge (Figure A-2) is a modification of the A.C. Bridge, where a variable resistor (optically coupled transistor) is added to the standard resistor $\left(R_{S} \Rightarrow R_{5}+R_{T}\right.$ (variable)). The off null of the HR-8 is filtered to altow essentially only D. C. changes (see Filter Box in a later appendix) and is converted into a negatively feedback current (by a $V \rightarrow I$ circuit, see Ref. 2) that changes the value of $R_{5}$ to balance the changes of $R_{X}$. By monitoring the current fed back, one has a means of knowing the changes of $R_{x}$ with a balanced bridge. For $R_{T}=1 \mathrm{~K}, R_{\mathrm{S}}=10 \mathrm{~K}$ one can get change in $R_{\mathrm{S}} \sim 30 \Omega$ for a current of $0+50 \mathrm{ma}$. By appropriate choices one can match the values of $R_{T}$ and $R_{S}$ to cover a sizeable range of self-balancing operation.

The A.C. Bridge was also used to measure the resistance of the superconducting films used in the experiment. One should note that at balance, there are number of sources of error, one being the current flowing in the film causing heating, and another being the lead's resistance from the film giving a potential drop and noise error in the measurentent. When one has very low resistances ( $\sim .5 \Omega$ ), It is difficult to get accurate readings with the above bridges, and hence, Kelvin Bridge (Figure A-3) was constructed. 


\section{SELF-BALANCING BRIDGE}

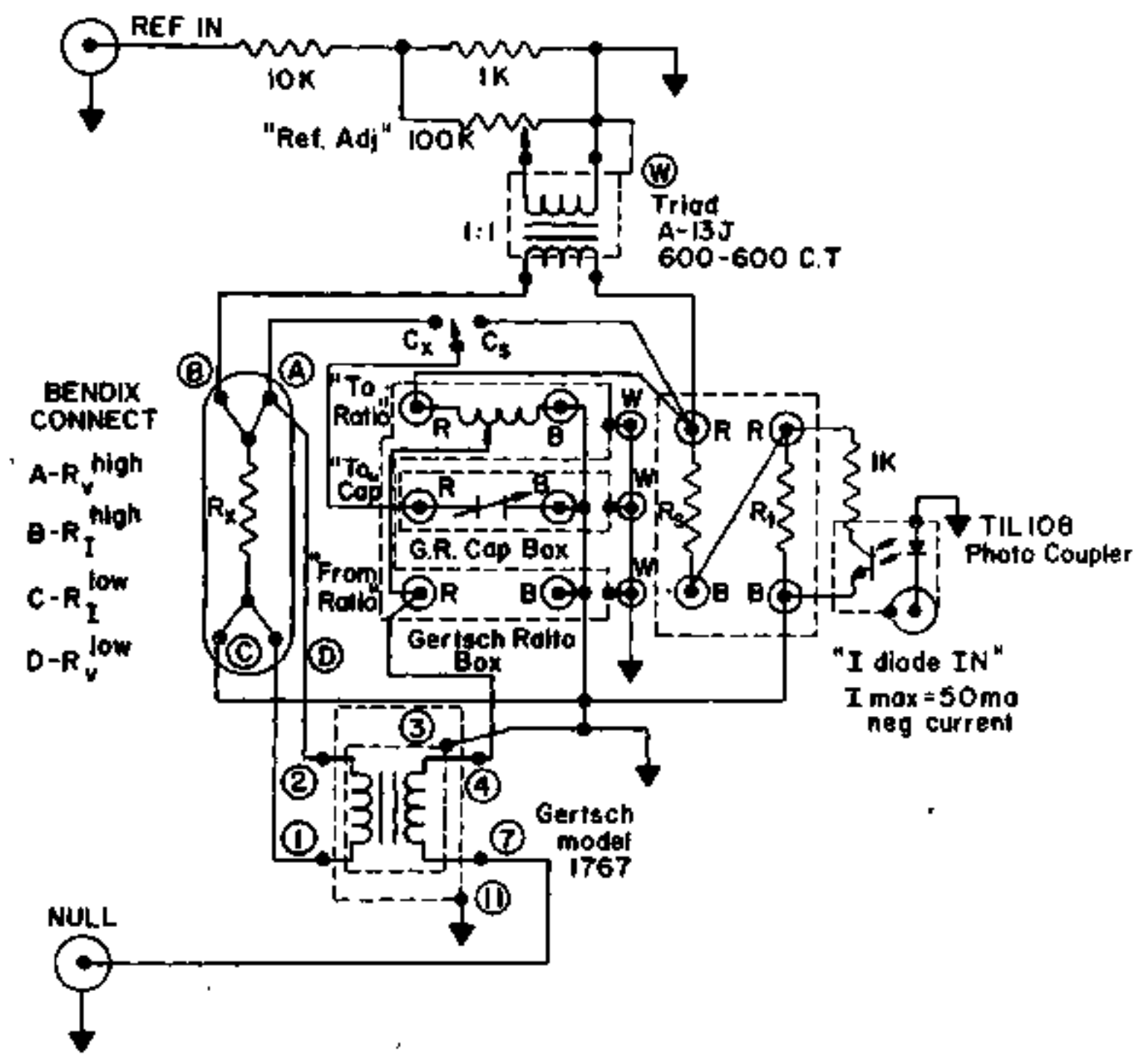

F1 gure A-2. 


\section{A.C. KELVIN BRIDGE *}

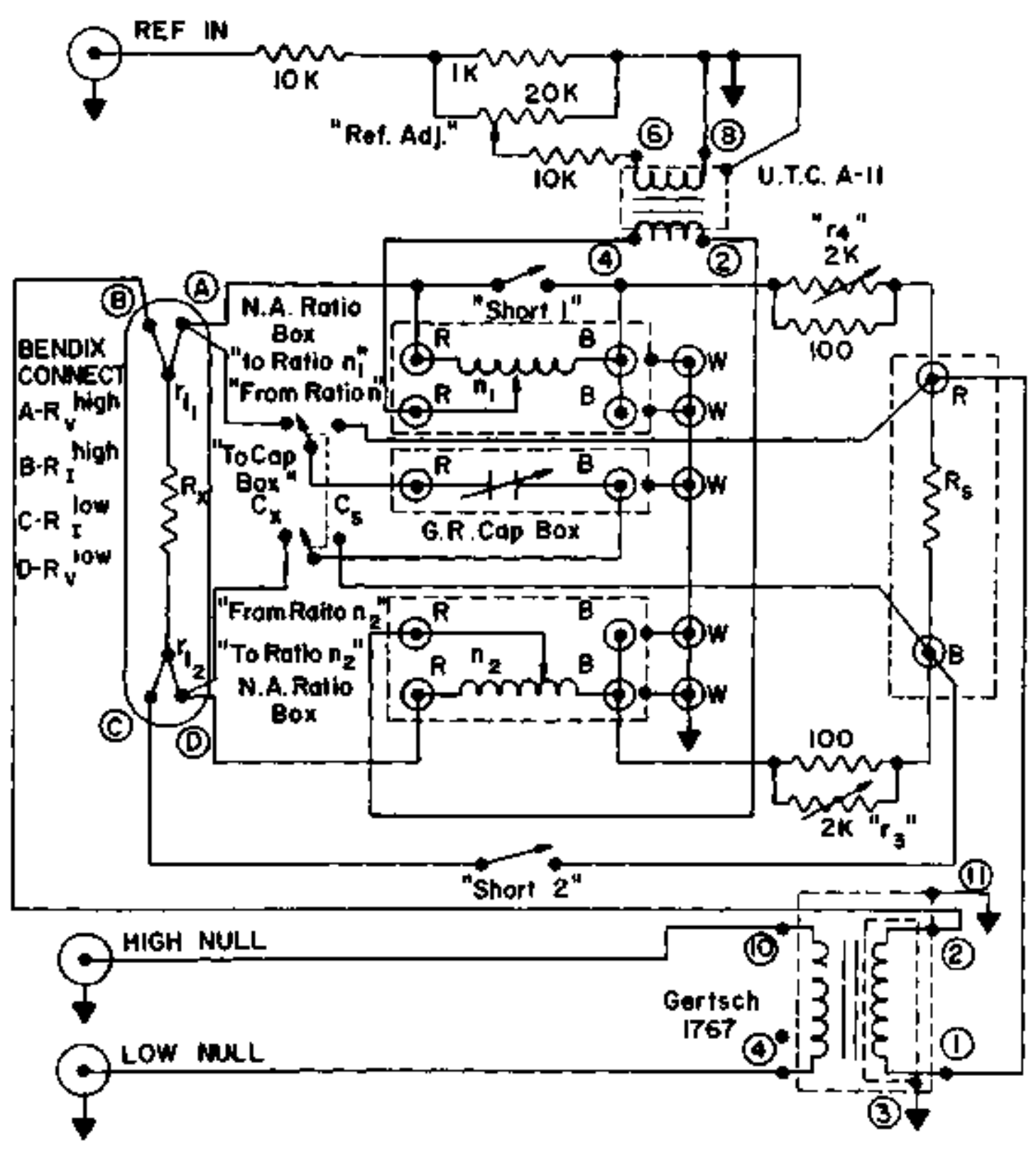

Figure A-3.

* Sometímes called a Double Ratio Bridge. 
This form originaliy was a resistance ratio bridge, but due to the sensitivity of A.C. phase sensitive detection schemes, and accurate ratio boxes (1pplo $0^{7}$ ) this bridge has been modiffed to an lapedance bridge. Hill and Miller ${ }^{3}$ did the orfginal work, whtch has eventually led to a commarclally avallable unit (although quite expensive) of similar design. ${ }^{4}$ With the commarically avaifable 16 bit $D / A$ converters, one could expand this idea to a self-balanced ingh resolution bridge. It should be noted that the bridge is symmetric under an interchange of the reference and detector. Also, at nutl the current in the unknown reststor is zero, and the lead resistances are nulled out of the measurement (but, changes in them are a large source of notse, i.e., bumping cables). The procedure for nulling is as follows:

1) Adjust rattos $n_{1}, n_{2}$ as for other A,C. bridge unt11 a nutl is reached with "short 2 " in place. Then

$$
\frac{n_{1}}{1-n_{1}} \approx \frac{n_{2}}{1-n_{2}} \approx \frac{R_{x}}{R_{S}}
$$

2) Put in "short 1" as well as "short 2" and re-nutl bridge 'by adfusting $r_{4}$. Then we have:

$$
r_{\ell_{1}} / r_{4} \approx \frac{n_{1}-r_{\ell_{1}}}{\left(1-n_{1}\right)-r_{4}}
$$

3) Take out both shorts and adfust $r_{3}$ for nul1. Then we have:

$$
r_{2} / r_{3} \approx \frac{n_{2}-r_{2}}{\left(-n_{2}\right)-r_{3}}
$$


4) Put"short 2" back in and readjust $n_{1}, n_{2}$. This procedure is continued until ali steps give nulis. Then:

$$
R_{x}=\alpha\left(\frac{1-n_{1}}{n_{1}}\right) R_{s} \quad \text { where } \alpha \text { is the bridge factor. }
$$

One should always let $n_{1}=n_{2}$ in all measurements to speed up the process. There are ganged transformers that work quite well in place of $n_{1}, n_{2}$, so that one always has $n_{1}=n_{2}=n$. (An example is the Gertsch Model 1000 A. C. Ratio Standard). This bridge has an accuracy and sensitivity for low power dissipations $\left(\times 10^{-12}\right.$ watts) of Tess than $1 \mathrm{~m} \Omega$.

The Germaniumresistors were fitted to a logarithmic series in temperature using the caltbrated pofnts supplied by the manufacturer. A table was generated that ellowed resistance measurements to be looked up for their temperature equivalent in steps of about $8 \mu \mathrm{K}$. The carbon resistors and the superconducting filers were calibrated from the values of the Germanium resistor when both where in thermal equilibrium. These bridges allowed temperature measurements to better than $10 \mu K$ and resistance measurements to better than 1 part in $10^{3}$ (for low resistances $\sim 1 \Omega$ ) and to 1 part in $10^{5}$ (for high resistances $\sim 10 \mathrm{~K} \Omega$ ). 
Appendix B. Optical Systems.

Since the experiment involved optical heating, systems for heating the film, as well as detecting correct system operation, were constructed. Light Emitting Diodes (L.E.D.) were used first (being in the visibie region for ease of optical alignment, etc.). Their output was monftored in the low temperature cryostat with si photo-transistors, using the Detector Box shown in Figure B-1. The operation of these devices at low temperatures is discussed elsewhere (see Ref. 5). The diode and detector where optically coupled in a block of lucite, that was at $4.2 \mathrm{~K}$, and the emitted radiation was shone onto the sample via a plastic light guide (see Ref. 6). This system could heat the sample at high frequenctes $(\sim 100 \mathrm{KHz})$, at relatively high powers ( $\sim 500 \mu$ watts). The system had certain drawbacks that led to its termination. The LED's, whlle putting out high powers, are quite inefficient and dfssipate to the local environment $\sim 100 \mathrm{~m}$ watts of power which is quite unacceptable for a low temperature environment. They aiso have a threshold before they entit Itght, which limits them for low power use. But, their major drawbeck is that relatively large currents ( $\sim 50 \mathrm{mo}$ ) are introduced into a low signal Jevel environment and produce undesirable electrical coupling that is indistinguishable from optical heating, since the optical signal and the drfving voltage are at the same frequency. For these reasons the LED system was discerded. 


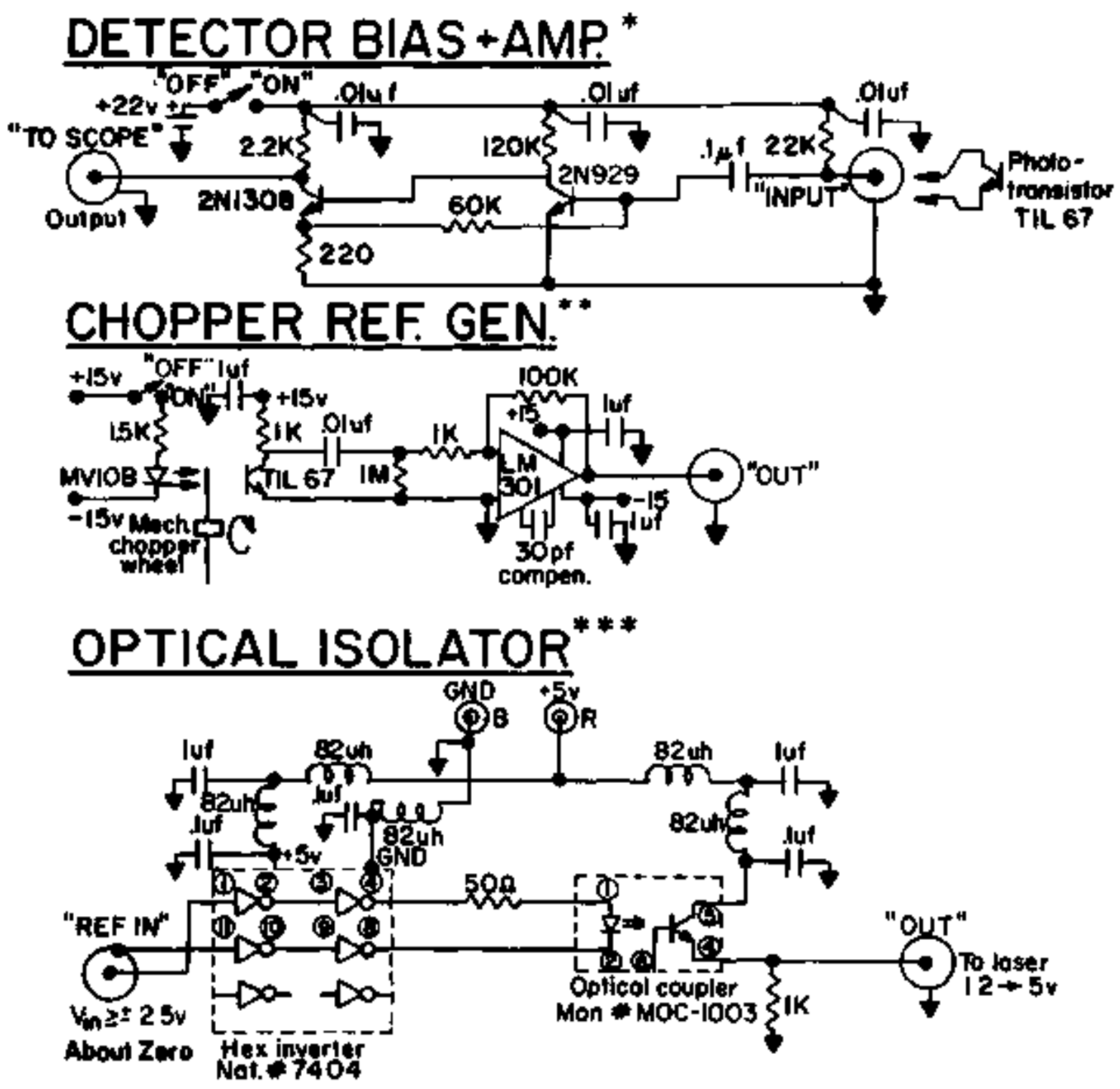

Figure B-1.

"See Texas Inst. Appl. Notes. "\#uilt by Dovid Winkler.
Built by Gary Wandersee. 


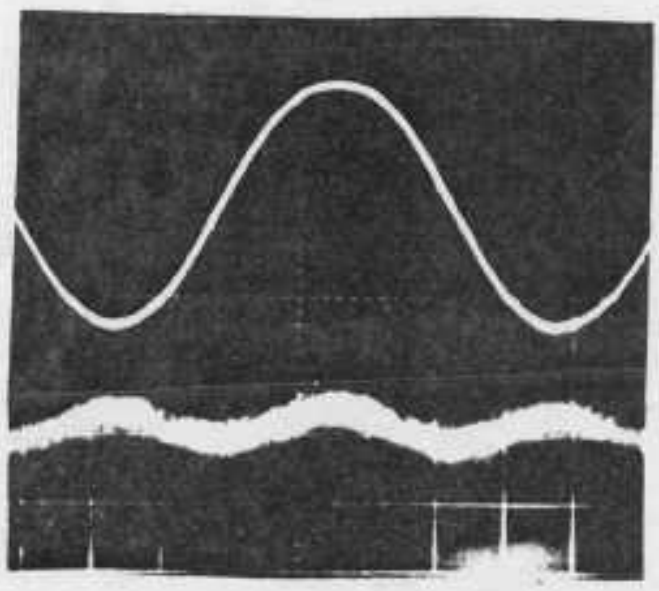

$T=300 \mathrm{~K}$

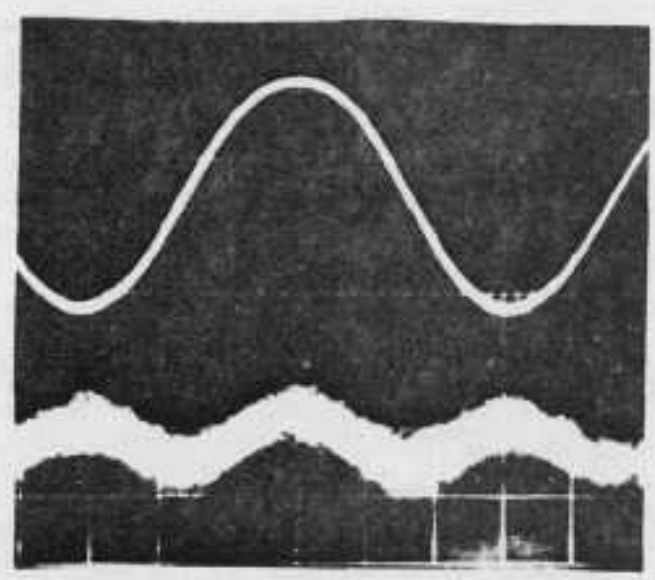

$T=77 \mathrm{~K}$

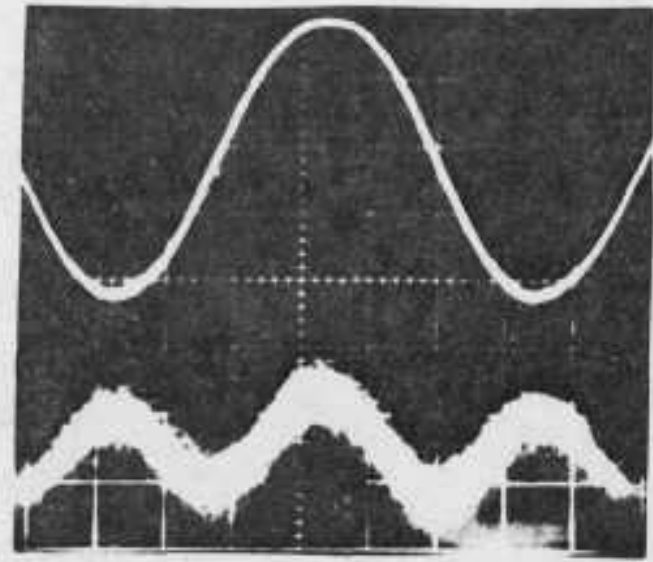

$T=4 \mathrm{~K}$

Figure B-2

Lower trace is the light intensity of light bulb (lover trace a $10 \mathrm{mv} / \mathrm{div}$ ) as seen by a detector (T1L67 Photo-transistor) at room temperature optically coupled to the light bulb via plastic light guide. The detector output was monitored with the circuit shown in Fig. B-1. The light bulb was driven by a constant voltage source (upper trace $02 \mathrm{v} / \mathrm{div}$ ) at a frequency of $10 \mathrm{KHz}$ (Horizortal axis (a $20 \mathrm{H}$ sec/div). There is little change in the light output for the three temperatures shown. 
The next optical system used involved very small tungsten filament light bulbs (see Ref. 7). The bulbs will operate at 4K and experience no problens in cycling to room temperature, It is necessary, however, to have the entire bulb heat sunk in some fashton (1.e., in an oil or LHe bath) in order that thermal gradients won't occur along the length of the bulb. It is belleved that this is the cause for observed bulb failure in gaseous He at $4 K$. The bulb has a continuous D.C. power output from zero up to about 400 w watts. When the bulb is operated in an A.C. mode, the power output is a combination of an A.C. Power and a D.C. power, with the ratio of A.C. to D.C. power decreasing linearly as a function of frequency, probably due to the inductive nature of the spiral filament (both physically and thermally) affecting the A.C. power, when driven by constant voltage source. This ratio falls off to about $10^{-4}$ at $10 \mathrm{KHz}$. When the bulbs are driven by a constant voltage source, the power emitted from the bulb, varies as the square of the driving voltage, and hence a driving frequency of $w$, witl be emitted at twice the driving frequency (i.e., $2_{w}$ ). Figure B-2 illustrates this point at three different temperatures, and shows that the Ifght emitted is at twice the driving frequency. This method was at first considered an asset, because the signal would be detected at twice the driting frequency, and thus reduce coupling problents encountered earlier. But, the aximum rejection of the detection methods was only an db, and thus a coupled signel at frequency $\omega$, detected at $2 \omega$, would look like a real $2 w$ signal. Extensive measurements reduced 
this coupling to a level where there was no longer any signal. It was because of the problem in distinguishing whether a detected signal was due to optical heating or E.M.1. coupling, that the experiment finally went to a room temperature optical source. The light was coupled via plastic light guide, that was thermatly sunk to the $4 K$ bath, and terminated in a copper sink from which it shone onto the sample. The light guide had an optical vaculan feed through that allowed separation of the light guide between the cryostat and the room temperature optical source. Duse to the transmission tharacteristics of the light guide (Ref. 6), the heat leak of the light guide was negiligible. This optical system reduces the entering light from the room temperature source by a factor of $10^{3}$. Thus, this reduction, together with the small light guide area, caused the need for a relatively large fiux room temperature source. The advantage of this method is that one can block the light entering the gutide, without changing the electrical structure, and thus distinguish between optical heating and electrical coupling.

A number of room temperature sources were used. A Targe movie light output was optically focused and mechanically chopped before it entered the light guide. A reference signal for the detection methods was derived using the circuit shown in figure B-1. This was three times the minimum light output needed, but the frequency of this optical source ( 1 knz ) was not readily adjustable due to the machantcal chopping nature. Since frequencies greater 
than I $\mathrm{KHZ}_{2}$ were desired, a large (10 mb) Helle laser was used that was modulated by varying the D.C. discharge current at frequencies up to $50 \mathrm{kHz}$. But, it had faherent stability problems, and due to its size (4 ft. long) had to be coupled over a long distance to the cryostat which resulted in considerable beam attenuation. Finally, D.C. modul ated I mik He-He laser (Metrologic Model \$382) was used. It could be coupled quite closely to the cryostat, and thus provided a variable frequency (0.C. $-500 \mathrm{KHz}$ ) light source of sufficient size to produce an optical heating signal. There were at first EMI coupling probtems due to the radiation of the D.C. modulating plasma in the tube, but an fsolator (FIgure B-I) was constructed that removed these problens. Thus, al though sone of the earl ier optical heating sources satisfied the heating requi rements, the modulated He-He laser, optically coupled to the sample, provided the most versatile and convineing source. 
Appendix C. Cryostat and Isolstion.

Because the experiment involved measuring very low level signals, particular attention had to be given to electrical isolation. But also, because a vibrating film in a magnetic field would generate nofse currents, isolation from vibration. and mognetic fields also had to be provided.

Figure C-1 shows the basic dewar system with the different Isolations. Vibrational isolation was provided by using bellows coupling links for 111 hoses to the dewar stand, and by mounting the stand on a vibration isolatfon platform. Tests were made using step hamner pulses as a driving force, and an accelerometer as a detector. The springs on the lower mount isolated frequencies up to about $200 \mathrm{~Hz}$ by a faetor' of 20. The "waffle" pad isolated frequencies up to about $600 \mathrm{~Hz}$ by a factor of 2 , and the foam pad-lead combination 1solated frequencies up to few KHz by a factor of 10. The system is essential $3 \mathrm{high}$ pass f1lters in series, with the last filter being the highest cutoff. This gives the overall system the ability to essentialiy reduce víbrations by factor of 400 for all low audio frequencies and below. The system does allow for direct accoustical coupling in the afr, but this proved to not be a noise problem.

Magnetic 1solation was achieved by three concentric high permeability shields (the third being directly in the He bath, to reduce doma in notse). Considerable isolation could be achieved by degaussing the shields. Although di rect A.C. 
All electricol nods RFI flliered.

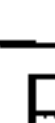
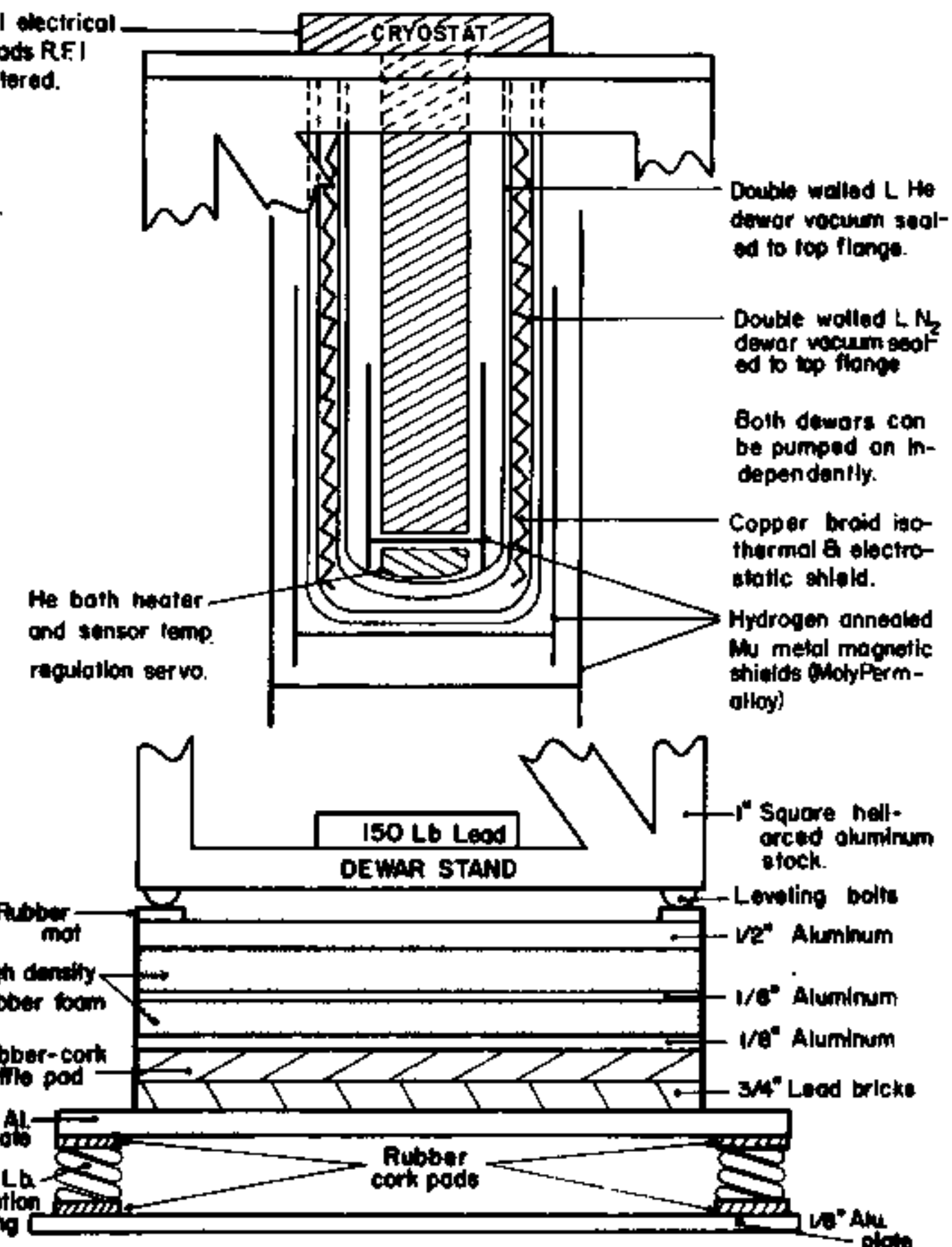
and sinser temp. ragulation servo.

FIGURE C-I 
currents in the shields, and low frequency (1 Hz) O.C. currents were tried for degaussing, stmple $60 \mathrm{~Hz}$ solenoid cot's on the shieids proved to work best. By using 3 variable transforaers in series, at peak currents of about 10 amps, one could slowly reduce the degaussing field to a negligtble mount. Best results were achieved by first reducing any manetization in $\$ 11$ three shields, and then degaussing from the outer shield first, to the inner shield last. Magnotic fleld (both transverse and axial) profiles were taken using a H.P. ammeter with a magnetometer probe (flux gate type). The field had two field reversals about the average mfnimum, which was about $7 \times 10^{-5}$ gauss at about $1 / 3$ the way up from the botton of the inner shield. This was also the region occupied by the sample when the cryostat was correctly positioned. It is interesting to note that the inner shield was removed for brief $t$ ime and $p l a c e d$ in the earths field, and then returned to its original position. It gained magnetization of about $1 \mu$ gauss/sec which would mean it would take about 10 days to accomplish a linear saturation in the earth's field.

The dewars were the usual double walled giass dewars. They were sealed to the upper flange by rubber washer seals to factlitate pumping on both the $\mathrm{LN}_{2}$ and LHe baths. Copper braid was hung around the He dewar above the ring seal, and allowed to be in contact with the $L M_{2}$ bath. This was to place an isothermal shield around the He dewar (with some E.M.I. shielding abilities) and to provide isothermalizing effect on the $\mathrm{LN}_{2}$ bath when it was pumped on. Due to the layered freezing effect when pumping 
on $\mathrm{LH}_{2}$ and the sequential "explosion" of the lower liquitd vapors through the frozen $\mathrm{N}_{2}$ crust, the bath was only slightly pumped on for test purposes, and was never done so during a run. The main purpose was to reduce vibrations from the bubbling $\mathrm{LN}_{2}$, but this never proved to be a probletil. The He bath was pumped to about $1.6^{\circ} \mathrm{K}$ and serwoed to about I $\mathrm{mK}$ with a carbon resistor-manganin heater combination.

Figure C-2 is the schematic for the He level detector system. The sensors were in a teflon tube that ran down the inside of the He dewar and was held in place with masking tape. The indicators were on the front stde of the dewar stand and at the same spatial points as the sensors. Thus, one could see when there was no longer LHe above a certain level by switching to the appropriate sensor. The system only needed minor trim adjustments over a two-year period of use. The indicators were essentially 100\% accurate.

The cryostat (See Figure C-3) consisted of an outer 0,F.H.C. copper can that was ibmersed in the bath. All ieads entering the can had been electrically tsolated using sub-mfniature "pi section" 10w pass filters" that gave about 60 do filtering above $5 \mathrm{MHz}$. The leads were in di rect contact with LHe and pass through epoxy feed thrus (Stycast) into the outer can. The inser can was attached to a copper tube thermally tied to

"They were a combination of the Allen Bradley "pi section" k1t CK68-5N in series with Lenox-Fugle 82 ph shielded inductor. This design was suggested by $W$. Whymann. 


\section{He LEVEL DETECTOR}

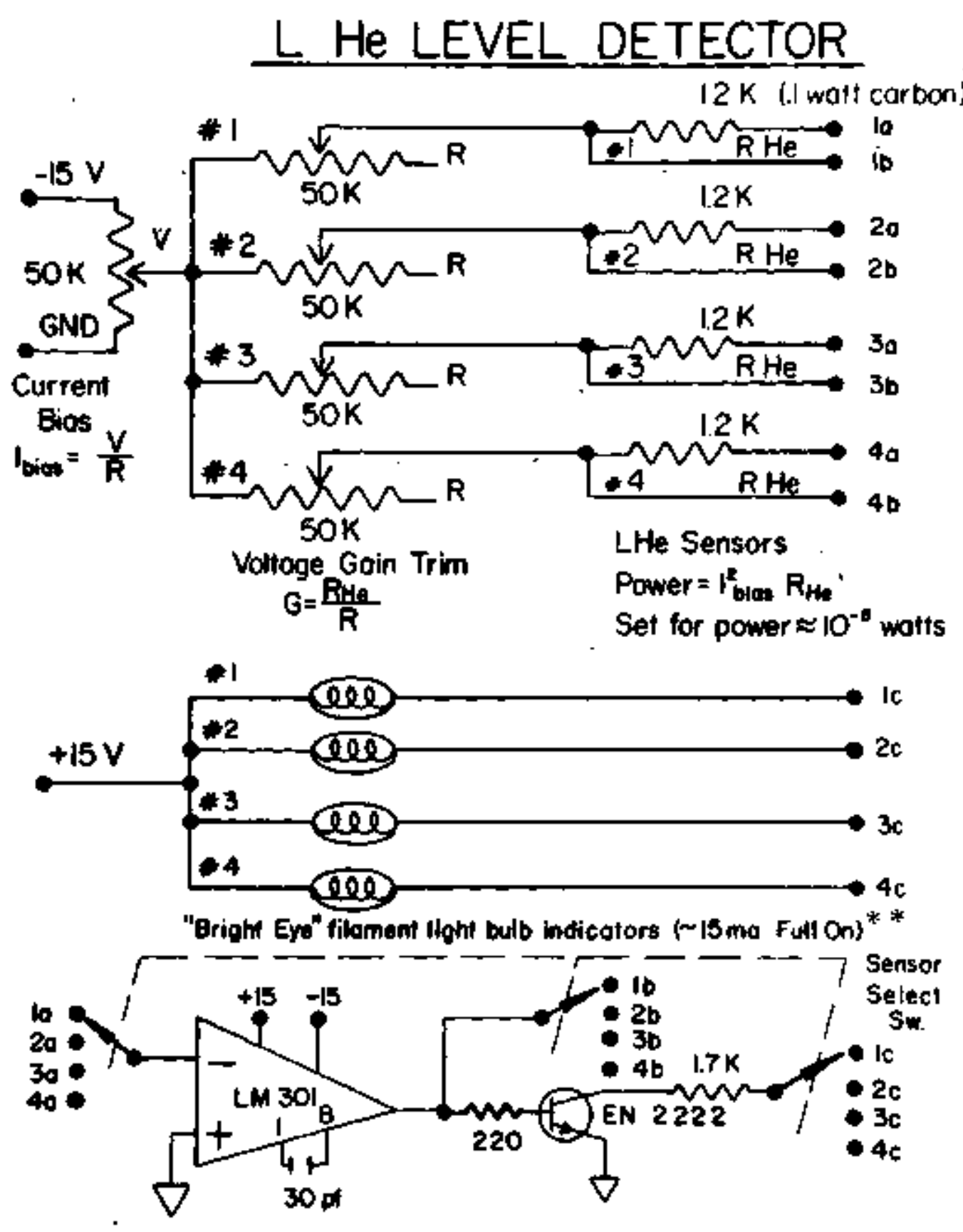

OPERATION: Goin Trim is odjusted for light"just on" with sensor in $\mathrm{L} \mathrm{He}$ with porticular current bias setting

* Resistors are inside Tefion qube on side of He dewor * * Dispioyed on dewor siond

Figure C-2. 


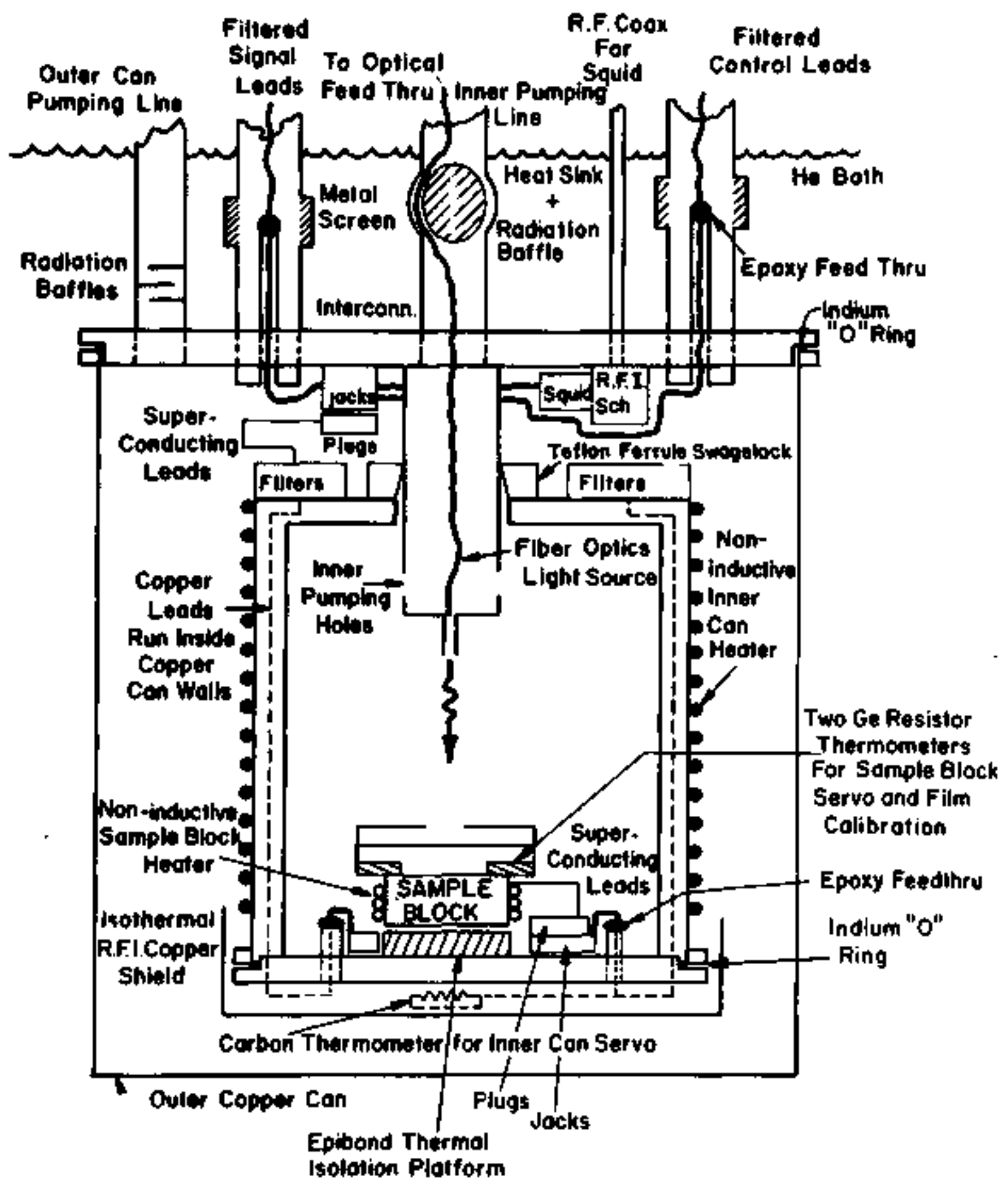

Figure C-3. Cryostat for Experiment. 
the bath by "swage lock" with a teflon ferrule in order to provide themal isolation, and facilitate optical focusing of the light source onto the film sample. The fiber optics used to conduct the 11 ght from the room tenperature source, ran down the inside of the inaer can pumping line and was thermally tied to the bath. The SQUID systen was completely shietded (see SQUID section) so that no R. F, could "leak" into the low level stgnal envfronment. To further factlitate this, the inner can was compietely electrically shtelded, and all leads entered second set of filters that gave attenuation above about $10 \mathrm{MHz}$ at $4^{\circ} \mathrm{K}$. The inner can was thermally isolated from the outer can (the one in contact with the bath) by using superconducting leads. It was independently sorvoed to provide temperature regulation independent of the bath. Inside, and thermally isolated from the inner can by an epoxy platform and suparconducting leads was the sample block. It was positionad directly below the light source. It had two independent Ge-resistor thenmoters (Cryo Cal). One was used for temperature calibratfon and the other was used with a manganin heater to servo the block. Since $80 \%$ of the heat is conducted to those sensors through their wires, care was taken to insure accurate readings. The copper leads were spllesd to superconducting leads and pressed In a copper-lense paper sandwich and bolted directly to the sariple block to insure good thermal contact. W1th both cans evacuated one could achieve thermal isolation times of about 20 minutes from the Inner can to the outer can and a few hours from the sample block. 
to the outer can. The block could be regulated to about $10 \mu k$ or better by the servo system as independently measured with the second Ge-resistor.

All measurenents were made inside an electrically shielded room. The cryostat and dewar assenbly allowed the experiment to be performed in a noise free environment with excellent magnetic, thermal, vibrational, and electrical isolation. 
Appendix D. SQUIOS

The SQUtD (Superconducting QUantum Interference Device) is a very sensitive device for measuring magnetic fields. In this heat capacity experiment it became obvious that in regions of low thermal sensitivity ( $\frac{d R}{d T}$ film was small) the voltages, corresponding to A.C. heating of the film, would be very small. Typlcally for a dR/dT $\sim 1 \Omega /{ }^{\circ} \mathrm{K}$, A.C. heating of $10^{-5} \mathrm{o}_{\mathrm{K}}, \mathrm{D} . \mathrm{C}$ : b1 as current of $10^{-6}$ amp, one is trying to measure 10 pico volt signals. For this reason a SQUID system was desired. At first, SQUIDS were made and adjusted without success. Then, a commercially zuailable system carse onto the market and was purchased. This system was improved, but finally abandoned for conventional electronfics due to problems discussed later. This appendix w111 not attempt to report on SQUIDS in general, but only as used in this work.

A SOJID is basically a weak link or region with "partial" superconductfuity connected in series with a superconducting ring. Flgure D-l shows a weak línk, which can be anything from two screws touching (point contact) to an anodized or scribed thin film (bridge). This weak link can be characterized by current equation that is similar. if not identical to that obeyed by a Josephson Junction. The equation expresses the relationshtp between the phase differences of the order parameters of the superconductors joined by the link $(\theta)$, the maximum current that can flow through the link at zero voltage $\left(i_{c}\right)$, and the 


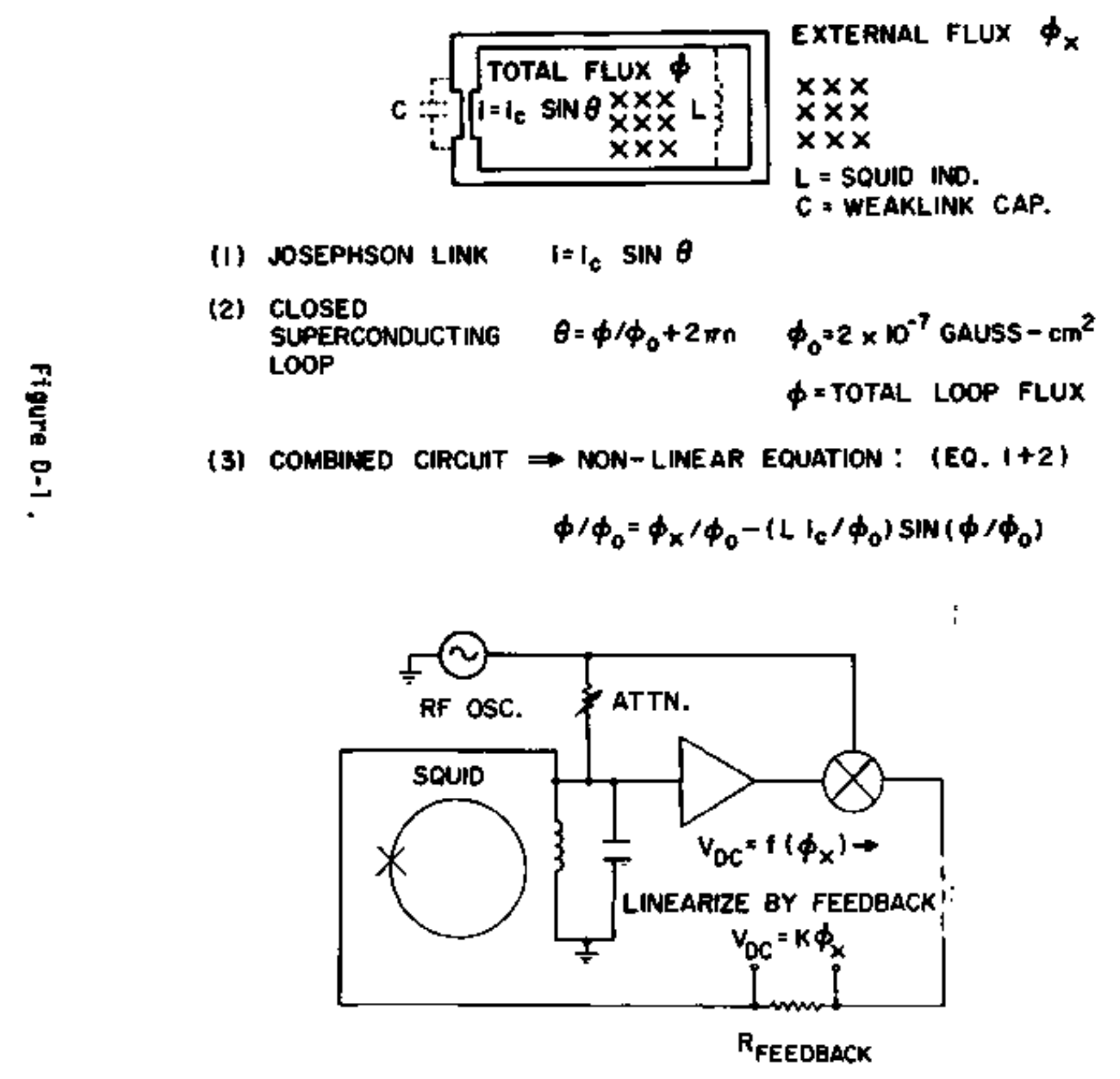


actual current in the link $(i)$. There is a capactitance associated with the link that will be left out of this introductory discussion. The link is then in series with a closed superconducting loop (with some inductarice L), and thus the phase difference around the loop must be related to the total flux in the loop (\$) which is defined modulo $2 \pi$. By combining these two requil rements. a non-linear equation relating the total fiux in the loop to the externally applied flux is derived.

In the real world, one wants to measure changes in the total flux of the loop (coupled in by currents from external experiments). One mathod of doing this is to weakly drive a tank circult with an R. F. signal where the coil is placed in the loop. This will cause changes in the total fiux to be reflected in a mon-? inear way in the level of the R.F. driving voltage. By amplyfing and mixIng out the $R, F$. diriving voltage, one obtains a non- 1 inear voltage function of the total flux in the loop (See Figure D-1). By taking the average of this output voltage and feeding $1 \mathrm{t}$ back as - current (through a high impedance) to the tank inductance, one completes a feedback $100 \mathrm{p}$ to the superconducting $100 \mathrm{p}$, and thus Iinearizes the voltage function of the totat flux in the Ioop. Then, by monftoring the feedback current, one has a direct measurement of the total flux in the loop, within the bandwidth of the feedback system. There are many treatments of not only the models for the SQUID, but mechanical analogs for the 
non-linear equations and other SQUID biasing schemes in the ifterature, 8

The particular SQUID used in this experiment was of the symotric design. ${ }^{9}$ It was made of either $\mathrm{Fb}$ or NbTi, was about 1" Iong and $3 / 8^{\prime \prime}$ in diameter (see Figure $0-2$ ). The point contact (or weak link) was made by a blunt and sharp pofnted set of 000-120 serews. The SQUID contains two holes that form two astatic loops, such that the current in the SQUID responds to only gradients in external flux. This feature, along with the superconducting cylindrical extension, allow the SQUIO to be scmewhat immune to local magnetic noise. Biesing and external signals are coupled into the SQUID through coils placed in the noles.

As mentioned earlier a commerctal SQUID system was purchased, ${ }^{10}$ which included an adjusting $k 1 t$. One of the major drawbacks of the SQUID outlined above is that precise adjustment of the point contact is needed in order for the SQUID to behave as weak link. The S.H.E. system measures the inductance of the SQUID at room temperature, and by setting the screws at a pressure, such that the change of the inductance is about $1 \%$ relatfve to the contact fully open, the SQUID has a reasonable chance of being in adjustment at $4 \mathrm{~K}$. By the use of a probe, it can then be tested in a He storage dewar to verify correct adjustment. By this method one can get an adjusted SQUID on the average in about two to three attempts (1though sometimes it may take 15 adjustments). Some tricks to mention for success are: 1) blow the gas through the 


\section{SYMMETRIC SQUID (Nb or NbTi)}

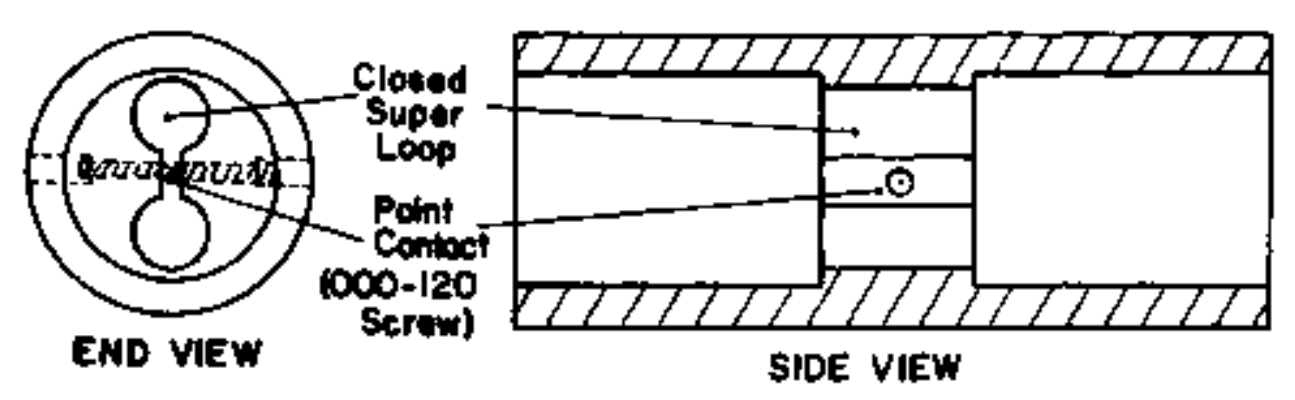

\section{SQUID BIAS}
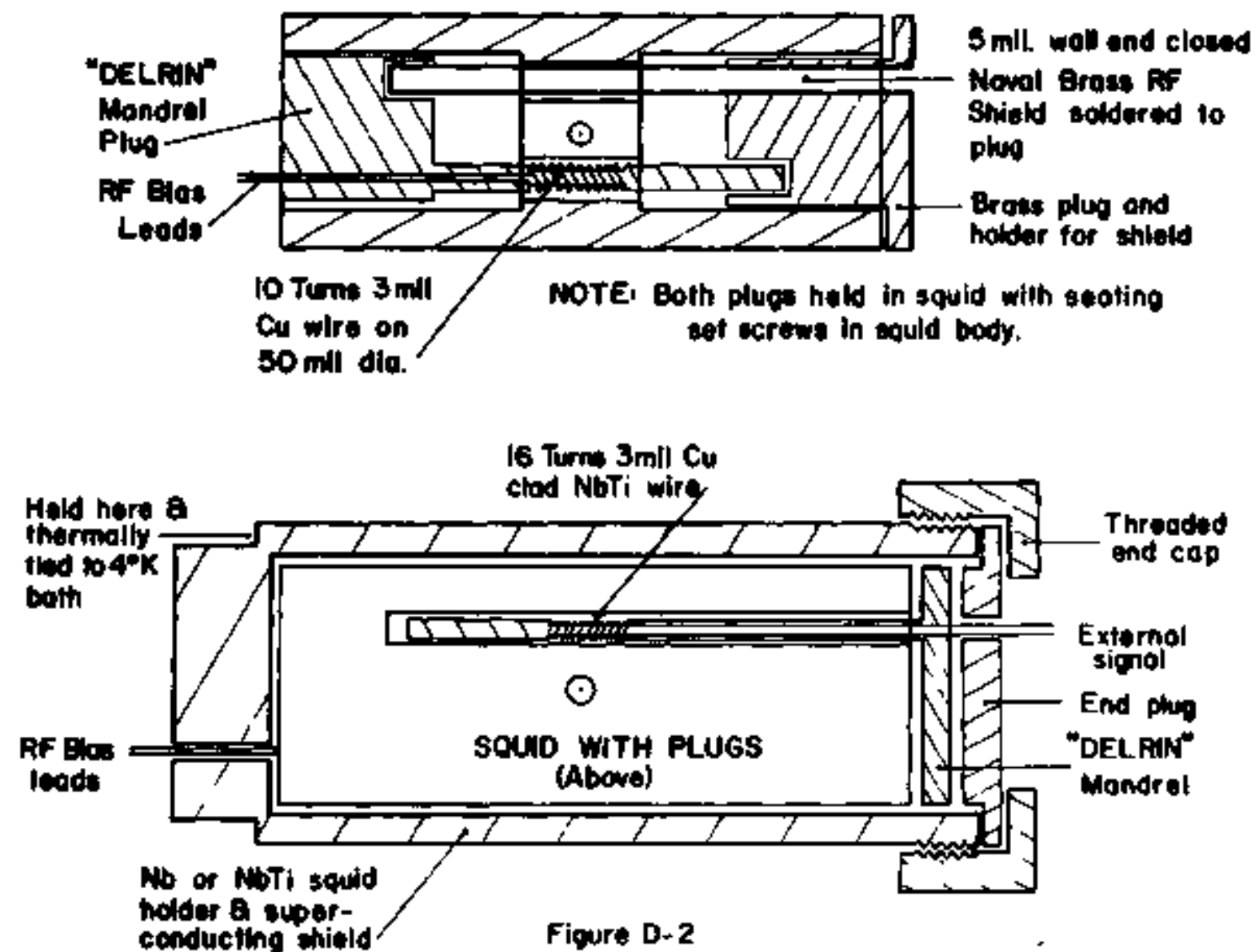
SQUID Just before entering the storage dewar; 2) take at lesst 5 minutes to enter (and leave) the storage dewar; 3) enclose probe in a bag of He gas upon exit from dewar until it has warmed to room temperature; 4) the pointed screw should be a "blunted" not a "tapered" point so that it doesn't dimple the flat surface or crush itself upon contact. These precautions (along with others mentfoned later) will aid in removing thermal effects on the adjustment of the SquTD.

Sone basic modifications were done to arrive at the SQUID system developed. First, the SQUIDS used on this experiment had a locknut for the screws that had a smooth flange so that upon "locking" the nut, pressure would be applied evenly, and not disturb the screw setting. Tests with the SQUID system indicated a need for shielding of the RF bias from the experimental signa] leads. Figure D-2 shows the symretric SQuID with "Delrin" holder for the bias R.F. coil, and a stmi Tar brass plug that had a thin-walled tube, to shield against any R.F. leakage out of the SQUID and back into the external signallead coil which was coupled into the other hole. The whole SQUID was in supperconducting tube that could be mounted at one end in any position in the cryostat. This brass sleeve shielding scheme is similar to that used by the Comell Group ${ }^{11}$ except their enclosure was also fijled with the gas and sealed off. The system described above was in a vacuum exchange can, and thus did not need to be sealed off.

There were some basic problems with the SHE electronics bias 
package that led to the construction of a new electronics system. First, the SHE package had a "Pierce" type oscillator with a third overtone cut crystal. Thts type of osctilator is primarily used for a fundamental cut erystal and consequently the SHE package would frequently Jump to $1 / 3$ the frequency of RF oscillation. Also, this oscillator put out rather large level stgnal ( $\sim 3 v$ ) that was difficult to attemate to get the Jow bias level needed for the SQUID. The SHE package used a rather crude. means of a varying ground plane to adjust and attenuate the level. Figure D-3 shows the improved electronics package. By using a tunnel diode oscillator, one maintained a constant frequency source at a much lower level and with a variable gain anplifier feeding capacitive divider network, a ten turn pot could be used to vary the R.F. level precisely over any predetermined range (ex. ten turns to the first R.F. D.C. field pattern).

The SHE package also used a cascode type preamplifier that feed a series of capacitive couplad R.F. op-amps and was detected with a peak diode detector. The cascode preanlplifier has an inherent feedback scheme (Miller effect) that causes selfosclllations due to the capacitive coupling in the transfstor from the output to the input. By using a dual gate MoS-FET (capacitive coupling $\sim 1 / 20$ of normal FET's) for the cascode circuit and using good shielding techniques, this effect could be reduced significantly (See Figure D-4). Also, an inductively coupled R.F, opamp stage also reduced noise coupling. Finatly, a synchronous mixer stage was used (see figure $0-3$ ) to detect the R.F. output, 


\section{SQUID ELECTRONICS}

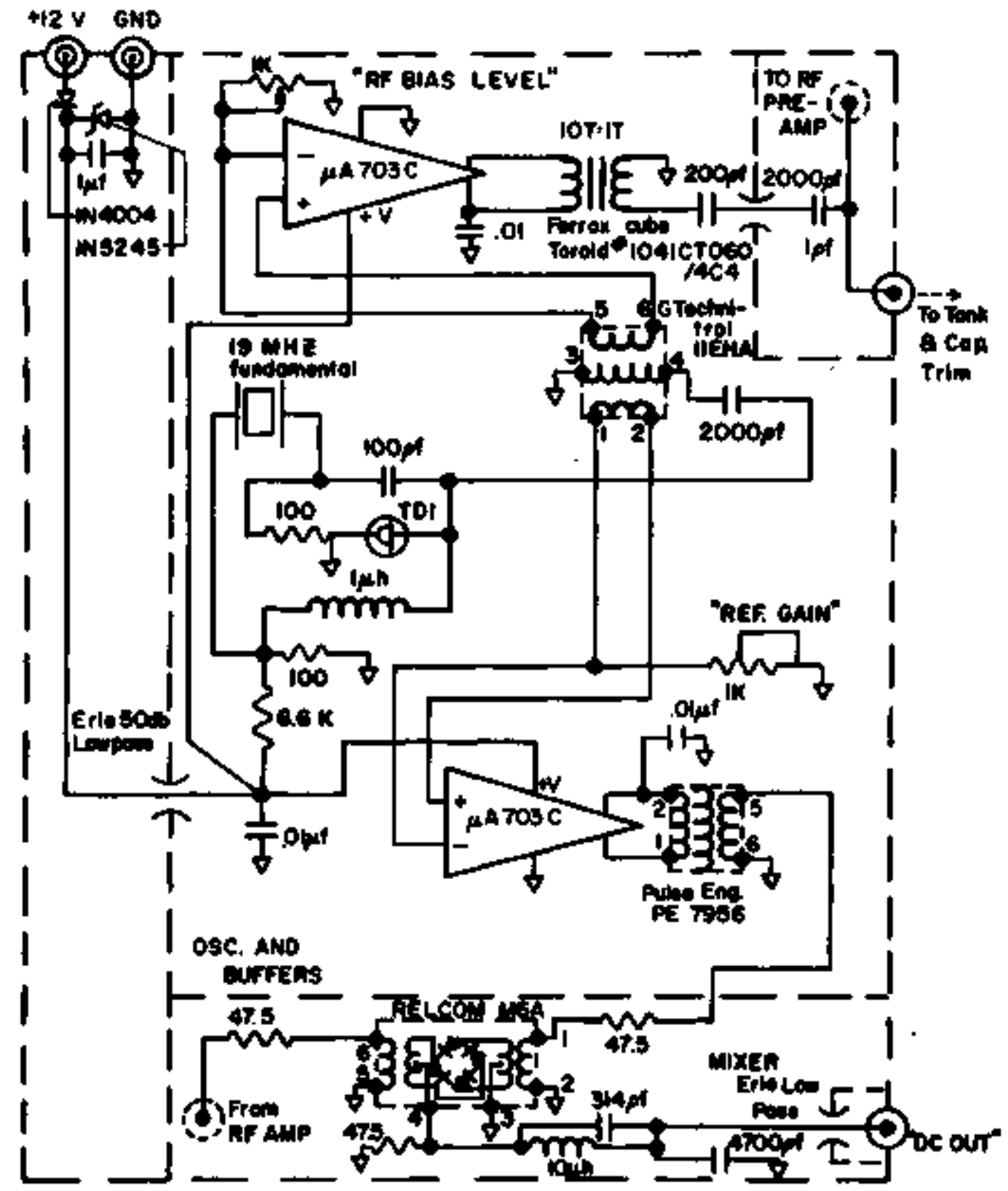

MIXER \& OSC. SIDE NOTE All circuils ore on copper cled plass boord.

Figure $0-3$. 


\section{SQQUID ELECTRONICS}

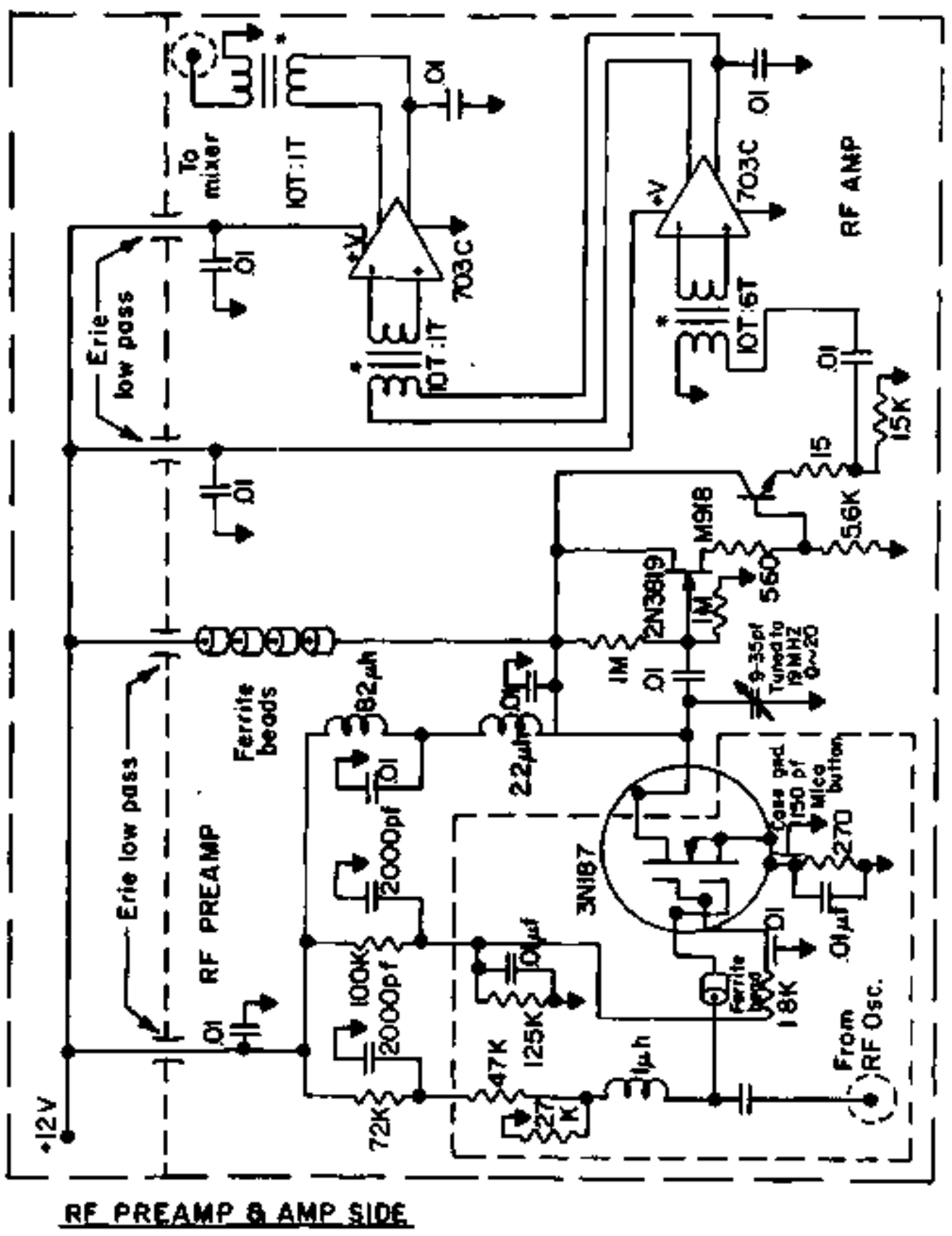

Figure D-4. "See Figure 0-3 for toroid core type. 
that, while it had a sonewhat reduced average value (not peak detection), it had an increased signal to noise ratio. The whole system was mounted in two sides of a carved aluminum box. It has an overatt gatn of about 15,000 in the R.F. ampliffer, with a $Q$ of 20 and with a peak to peak notse of less than about $1 \mu v$.

The SCuID was to be used as a null detector in a bridge circuit (See Figure D-5, "Access chimney"). A standard resistor would be in series with the superconducting f11m resistance and the sensing coil in the SQUIO. By biasing the film from a constant current source $\left(I_{0}\right)$, and using the off null of the current in the SQUID coll (fed into the"D.C.IN" part of Figure D-5) to set the lever of a counter current $\left(I_{F}\right\}$, one then has at null the relation that the ratio of the resistances, $R_{s t a n d a r d} / R_{f i l m}$, is equal to the ratio of the currents, $I_{0} / I_{F}$. This allaws one to use the feedback circuit of Figure 0-5 to continuousily measure the filn's rasistance (at "R out"), providing one knows the biasing current $\left(I_{0}\right)$ and the standard resistance. The standard resistor was made of a short length of manganin wire. All leads were superconducting and each leg of the bridge was shunted by a low valued N.P.O chip capacitor to reduce any R.F. leakage from the SQUID onto the film. The whole bridge was at first built in an enclosure like the one around the SQUID, with leads running to the SQUID and the film, as well as to the "Feedback Box" of Figure D-5, which was mounted on top of the cryostat. Later the bridge was reduced to in line splfces of the wires directly on the film sample block (see sample preparation and eryostat sections). Because the 


\section{SQUID D.C. BIAS AND FEEDBACK BOX}

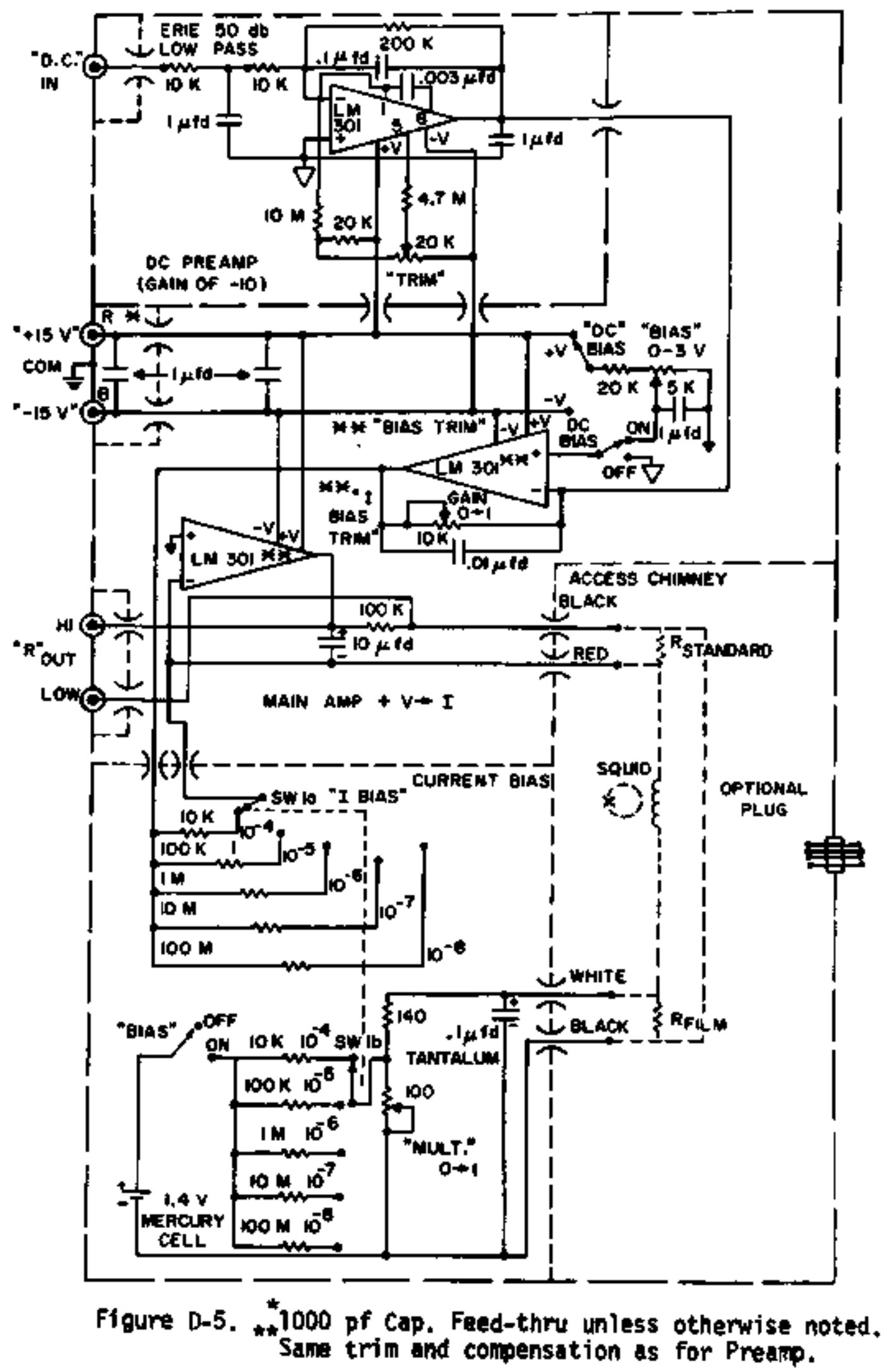


procedure of the experiment was to measure both the film's resistance and an A.C. heating component of the $f f 1 m^{\prime} s$ resistance, the feedback current wuld have to be analyzed not only for its D.C. value but also for the A.C. heating component.

Problems developed with the 5quID system as outlined above, that eventually led to using conventionat alectronics in the expurfment. The first problem was that of the inherent mixing in a SQuID of any two 51 gnals due to the non-linear current equation. Mixing was found between any two frequencies used in separate and independent feedback schenes. For example, D.C. current could linearly vary a phase sensitive detected $1 \mathrm{KHz}$ signal by $180^{\circ}$ (i.e., from full positive to full negative maninum detected output). This is essentially like varying the phase at which the P.S.D. Is demodulating. Also, frequencies of $10 \mathrm{w}(50 \mathrm{~Hz})$ and high $(10 \mathrm{KHz})$ values would mix with each other. This problem would make independent determination of the filas resistance (D.C. or low frequency), and the heating component (A.C. or high frequency) quite difficult. One mathod around this suggested much later by Kamper (at N.B.S. in Boulder) was to use a high frequency carrier ( $\sim 150 \mathrm{KHz}$ ) and feed it Back with ail other signals riding as side bands on this carrier and detected in the fedback loop.

A nore inportant problem that developed was the fact that the critical current of the weak link $\left(\dot{i}_{c}\right)$ was temperature dependent. The SquIDS were adjusted at $4.2 \mathrm{~K}$ in a storage dewar, but were used In a cryostat at about $1.5 \mathrm{~K}$. This changed the critical current behavior enough to make the 5QUID inoperative at the desired 
operating temperature $(1.5 \mathrm{~K})$. Other workers have clafmed both Immuntty to this problem and similar difficulties as mentioned above. The safest solution to the problem is to have a lever arrangement to adjust the contact in sftu. The basic behavior observed was a dramatic increase in the critical current of the contact $\left(i_{c}\right)$ as the temperature was lowered. Reference 8 mentions this behavior and also references the work of Strait. ${ }^{12}$ strait's conclusion was that "dirty" contacts (heavily oxidized) had mechantcai stabijtty, but a large temperature dependence in the critical current, while "clean" contacts (honed surfaces) had little temperature dependence,but mechical instabilities. Because of the anbigurous behavior of the adjusted point contact, a run was made, mont toring the SQUID characteristics of a set of NbT1 contacts that had been oxidized for 2 hours at $500^{\circ} \mathrm{F}$ in afr. When Sojios are R.F. biased, one cannot independently determine the critical current $\left(i_{c}\right)$ since it is related to the kinetic inductance of the SQUID (L), and to the mutual inductance between the SQUID and the R.F. bias coil (M). The measured quantity is the average R.F. voltage level on the tank $\left(\bar{v}_{1 c}\right)$, at a current just before the current in the loop surpasses the critical current, 1.e., at the first "step" in the usual staircase trace of a R.F. average voltage vs. R.F. modulated field. The distance between the steps in the stalrcase $(\Delta)$ is related to the number of flux Jumps and the mutual inductance (M). This does not independently determine the critical current of the loop as a function of temperature since the quantities $L$ and $M$ can be temperature dependent. During the test run with the SQUID, an 
Unusual feature was observed as the temperature was lowered. "Sp1kes" were observed $\left(A_{S}\right)$ at the onset of each step that can be related to further quantities in the experiment. From Reference 9 one can construct a set of three equations (See Figure $D-6$ ) that contain three temperature dependent parameters. In this way, the equations were solved by taking the experimentally determined parameters $\left(V_{1 c}, \Delta, \Delta_{S}\right)$ and then extracting the temperature dependence of the critical curreit (See figure $0-6$ ). This behavior was in . qualt tative agreement with that of Strait.

It was thus concluded that the reTiability of the SQUIDS used was questionable and in view of the complext ty of the experiment, and the problems of mixing and therefore ambiguous interpretation, 1t was decided to do the experiment with conventional electronics. 


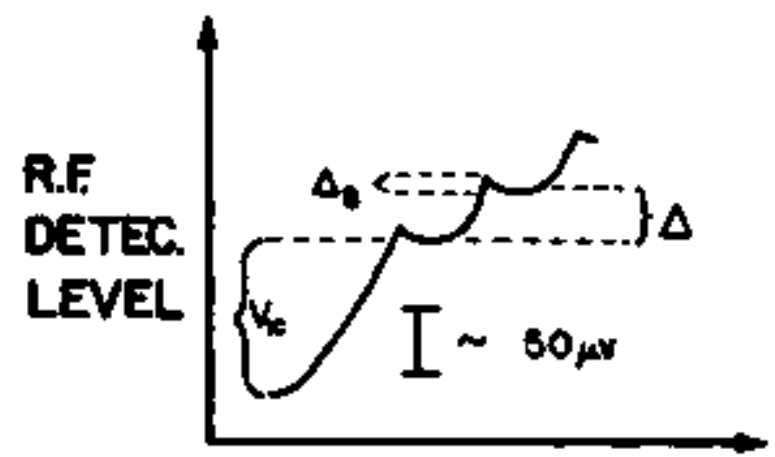

R.F MODUL. AMPL.
a) $V_{\text {Ic }}=\frac{\omega L L_{1} I_{0}}{M}$
b) $\Delta_{S}=\frac{\phi_{0} \omega M}{L}$
c) $\Delta=\frac{\omega \phi_{0} L_{1}}{M}$

NOTE:

Actuol troce of R.F level on oscilliscope Tomp. is $=1.46 \mathrm{~K}$

- From Pot. 9 $i_{\varepsilon}=$ CRITICAL CURRENT

$L=$ SOULD INDUCTANCE

$\omega=$ RF FREQUENCY .

$L=$ RF BAS COIL INOUCT.

$M=$ MUTUAL INDUCT OF RF BIAS COIL AND SOUID $\phi_{0}=$ QUANTA OF FLUX

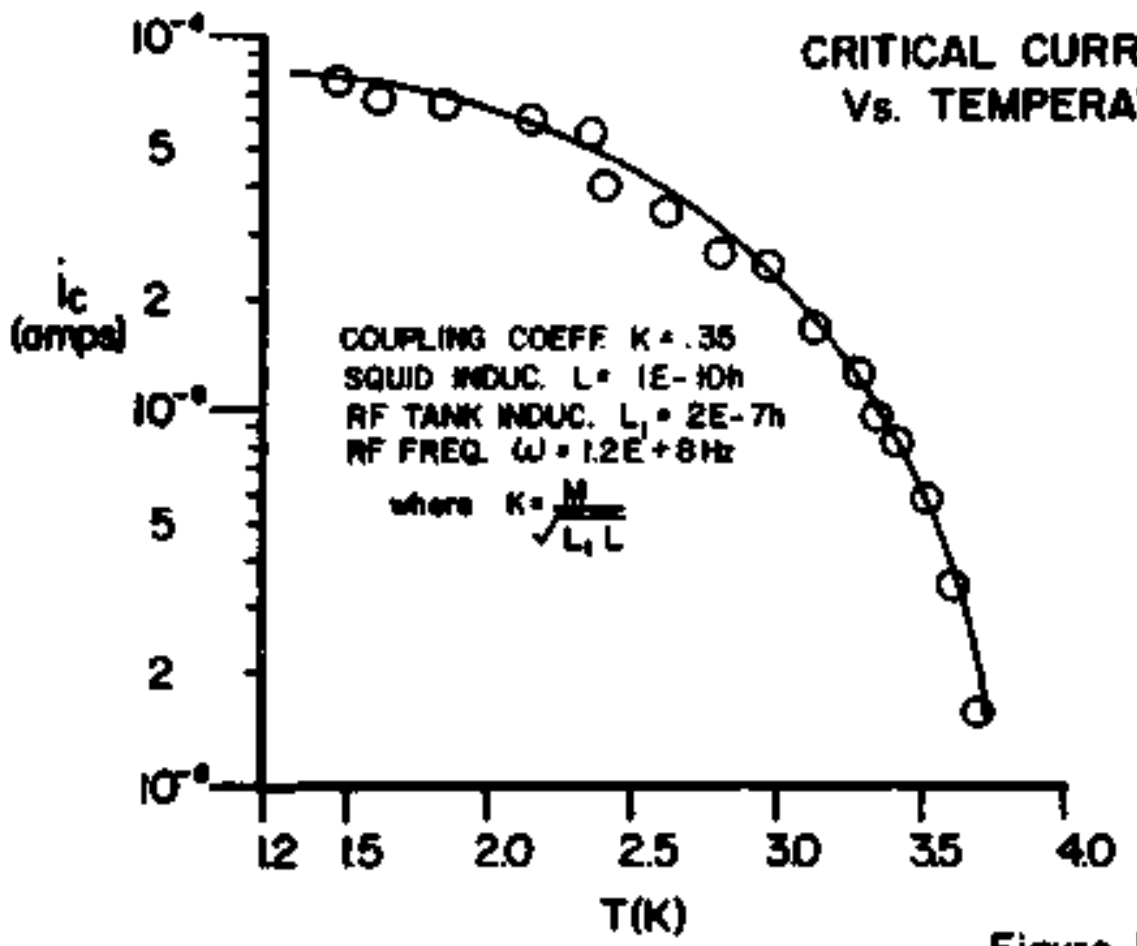

Fioure D-6

The upper graph shows the observed "spikes" in the normal staircase pattern of an R.F. blased SQUID. The lower curve shows the critical current $\left(i_{c}\right)$ ws. tenperature as determined from the three equations $(a, b, c)$ refating the three tempersture dependent experimental parameters $\left(V_{1}, A_{,}, \Delta_{5}\right)$ and an average value of the slowly varying parameters $(K, L)$ was used in the plot shown. 
Appendix E. Functional offferentiation and Numertcal Anatysis

In the experiment, the resistive transition of the thin superconducting film is used as its own thernometer. The fi个m's resistance is callbrated in temperature when it is in good thermal contact with a Germanturt thermometer, and thus from this calibration, the f11m's temperature can always be determined by knowing its A.C. resistance. A more subtle problem in the experiment is to determine the slight temperature rise (AT) associated with the A.C. optical heating, which produces a slight change in resistance ( $\Delta R$ ) and creates an A.C. signal at the optical heating frequency. Using a Taylor series expansion about $R_{0}$, the average resístance value at any temperature where $\Delta R \quad<R_{0}$ for all values of $R$, we have:

$$
\Delta R \equiv\left|R(T)-R\left(T^{\prime}\right)\right|=\left.\frac{d R}{d T}\right|_{R_{0}} \Delta T+\left.\frac{1}{2} \frac{d^{2} R}{d T^{2}}\right|_{R_{0}}(\Delta T)^{2}+\cdots
$$
where $\Delta T=\left|T-T^{*}\right|$

A first order approximstion would relate $\Delta R$ to $\Delta T$ by:

$$
\Delta R=\left.\frac{d R}{d T}\right|_{R_{0}} \Delta T
$$

This is a crude approximation, since higher order terms are present in most resistive transitions. But, if the interval is smali over which the slope of the transition ( $\frac{d R}{d T}$ ) is determfned, then a línear approxination will work, and higher 
order terms can be neglected. The function $\frac{d R}{d T}(T)$, which is the slope of the resistive transition, was determined by a number of methods. Since in the experimental data taking process, the temperature is increased at constant rate in time ( $\frac{d T}{d t}=r$ (rate) ) from below to above the transition, the function that is needed can be modified to the function below:

$$
\frac{d R}{d r}=\frac{d R}{d t} \frac{d t}{d t}=\frac{d R}{d t} \frac{1}{r}
$$

It was independently veriffed that $r$ was a constant in time by plotting $\left(T_{t}-T_{(+1}\right)$ vs, time $(\Delta t \times i)$ to obtain a straight line, where $T_{i}(\Delta t i)$ was the digita] form of temperature. Three mathods were used to determine $\frac{d R}{d t}$ and hence $\frac{d R}{d T}$. The first two were analog methods that were hindered by analog notse. The third method was a digital technique that was hindered by digital noise due to the sampling. But, by using digital filtering techniques and a fine sampling grid (1020 points), the noise could be significantly reduced. The digital method was used in all subsequent data andysis.

Since the dynamical resistance is a voltage, linearly related to $R$, then $\frac{d R}{d t} \propto \frac{d V_{R}}{d t}$. Thus, one only has to differentiate this voltage $\left(V_{R}\right)$. The basic analog circuit for differentiation is shown in Figure E-1. It is simply a high pass filter, such that the fastly varying functions are not attenuated, and hence it is a differentiator. The problem is that noise pulses will also get 
BASIC DIFFERENTIATOR

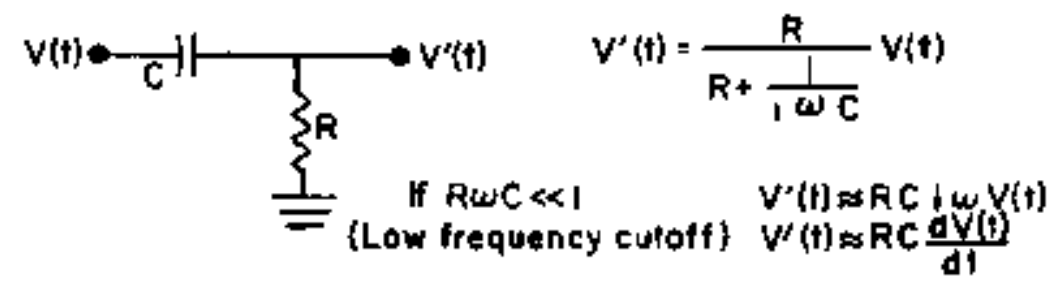

REALISTIC DIFFERENTIATOR

Includes high frequency culoff for $\omega \gg t / R_{1} C_{\text {, }}$

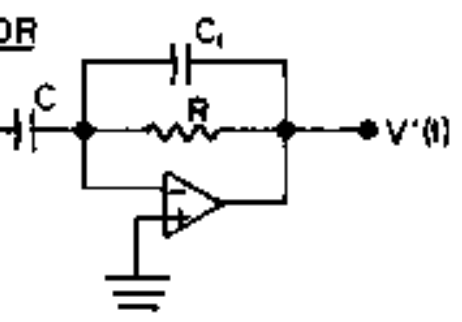

" tuneo oIfFERENTIATOR

1) Specify $\omega_{L}, \omega_{0}, R_{1}$

2) $R=\omega_{b} \xi A_{1} / \omega_{b}$

3) $C_{1}=\frac{1}{t} R \omega_{L}$

4) $c_{2}=2 \omega_{1} \xi c_{1} / \omega_{0}$

5) $\mathrm{C}_{3}=\mathrm{C}_{2} \cdot \mathrm{E}^{2}$

Where $\xi=0.65$

$c_{0}=$ High frequency cutoff

che Low frequency culoff

$R_{1} \pm$ input registance

* * TUNER DQUULLE DIFFERENTIATOR

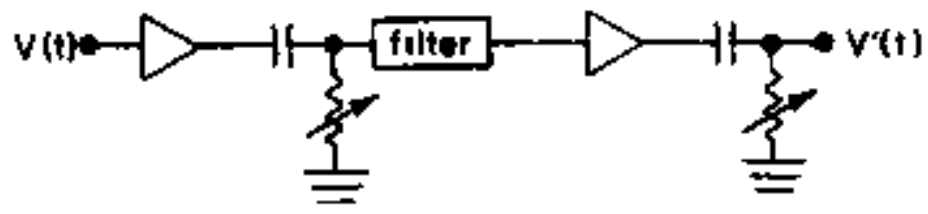

$V^{\prime}(1) \propto \frac{d^{e} V(t)}{d t^{2}}$ for some bandwidth of frequencies

Figure E-1.

Different and $\log$ methods of differentiating a time varying voltage with respect to time.

"See Ref. 13. ${ }^{\star \star}$ See Ref. 14. 
through the fijter, and thus the derivative of a signal includes the full bandwidth of nolse allowed by the filter. A more realisttc version of a dffferentiator is shown in Figure E-I, where a low pass filter is included to act as a cutoff on the high frequency noise. A more complete idea of using a cutoff is to have a tuned differentiator that only looks at a narrow range of the Fourier components of a derivative. Finally, one can do double differentiation with filtering, and then integrate the output. The first three filters shown were built and tested using andiog circuits. The major limf tations were that the signat to noise ratio was never very large $(\sim 20)$ and the maximum range of "frequencies" using conventional components was only three orders of magnitude (i.e., for $C \sim 10 \mu \mathrm{fd}, \mathrm{R} \sim 1 \mathrm{M}, \frac{\mathrm{dV}}{\mathrm{dt}}=10^{-4} \rightarrow 10^{-1} \mathrm{v} / \mathrm{sec}$; with higher ranges for lower values of $\mathrm{C}$ ).

A second method of analog dfferentíation involves using feedback or modulation techniques to extract the derivative of a voltage signal. The feedback method, shown in Figure E-2, involves positive and negative feedback. This circuit was constructed, but went into oset1lation (due to the positive feedaack) whenever the limit of true differentiation was met $(\alpha=1)$. This meant it still was an aproximation to the derivative of the signal. The second method shown in Flgure E-2 involves using P.S.D. (Phase Sensitive Detection). This method actually works quite well If one knows the form of the derivative. In principal one adjusts the phase $(\phi)$ of the reference channe] to let $\sin \phi=0$. 


\section{AMALOG PIFEERENTIATION QY EEEDBACK}

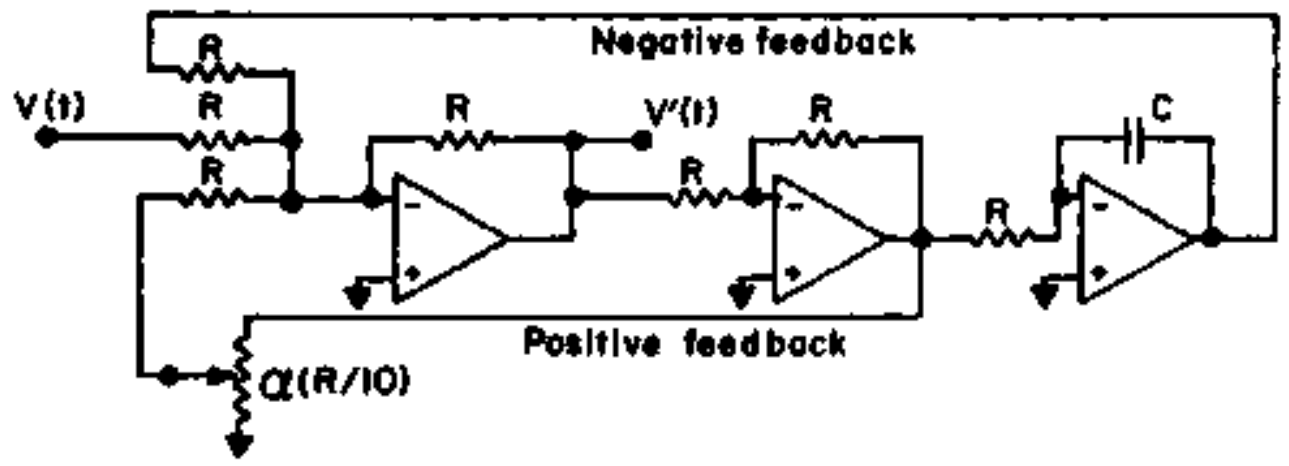

1) for $\omega \ll 1 / R C \quad V^{\prime}(t) \approx-V(t)+\alpha \cdot V^{\prime}(t)-\frac{1}{R C} \int V^{\prime}(t) d t$

a)

2) differentiate $\rightarrow(1-\alpha) \frac{d V^{\prime}(t)}{d t}+\frac{d V(t)}{d t}=-\frac{V^{\prime}(t)}{R C}$

3) Limit $f a+1 \quad V^{\prime}(t)=-R C \frac{d V(t)}{d t}$

\section{DIFFERENTIATION BY INTERMEOIATE MODULATION}

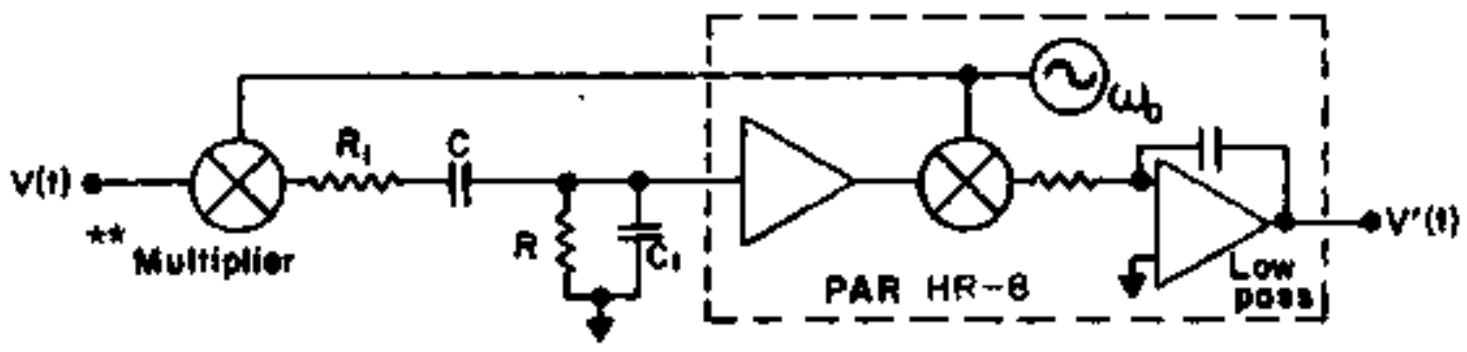

1) $\omega_{0} R, C \ll 1 \quad \omega_{0}=$ modulation freq.

b)

2) $\omega_{L} R C \ll 1 \quad \omega_{L}=$ sow freq. cutoff

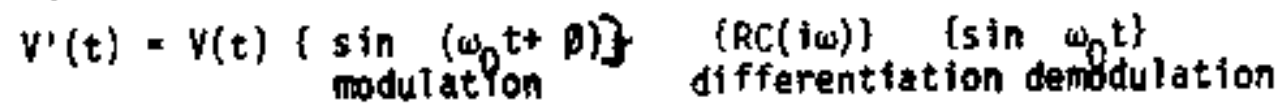

$\rightarrow V^{\prime}(t)=R C\left\{\frac{d V(t)}{d t} \sin \left(\omega_{0} t+\phi\right)-\omega_{0} V(t) \cos \left(\omega_{0} t+\phi\right)\right\} \sin \omega_{0} t$

3) After Low Pass Filter

$\rightarrow V^{\prime}(t)=R C\left\{\frac{d V(t)}{d t} \frac{\cos \phi}{2}+\omega_{0} V(t) \frac{\sin \theta}{2}\right\}$

Figure E-2.

More complicated analog differentiation methods. "See Ref. 15. " See Figure E-5. 
But; because of the large size of $\omega_{0} V(t)$ (especially at $h i g h$ frequencies) relative to $\frac{d V(t)}{d t}$, the adjustment of phase is crucial in order to renove any signal but the derivative. In practice, a d1fference of two tenths of a degree was enough to distort the derivative. The method of Figure E-3 is more complicated and is used primarily for tunneling wark where the voltage wanted is that which is Iinear to dV/dt (or higher differentials). But, when the function that is wanted is $\mathrm{dR} / \mathrm{dt}$, thien equations (E-3-5) through (E-3-8) show the diffleulty of obtaining the function $d R / d t$ without other derivatives complicating the problem. Nevertheless, this method was aiso tried without success. The final analog form tried was. that of modutating the voltage, which varied linearly with $R(t)$. Figure E-4 shows the derivation of the result that by detecting at twice the frequency of the vol tage. modulating square wave, the detected output would be proportional to the derivative at high frequencies. But, at high frequencies the modulation is non-linear, and there is some attenuation in the voltage paths of the experiment, so that this method aiso included other forms besides the $\frac{d R}{d t}$ function. Thus, in general, the analog methods are hindered by notse. The noise problens can be reduced at the cost of distorting the derivatives' waveform. Because the derivative of the transition was not known, one would be unable to detect distortion in the true derivative. For this reason, digttal methods were used.

The digital method of determining the derivative has the 
. F19ure E-3.

Hichor Order kornonic Oetection wethod"

Cirst nodulate resiseance $(R(t))$ w1 th a current such that:

$$
V(t)=V_{0}+A \sin \alpha t
$$

Tharefore, the current is: $f=f(V)$ which we assune is anslytic.

Then. expond $f(v)$ a a Tulor serles:

$$
f(v)=f\left(v_{0}\right)+f^{\prime}\left(v_{0}\right)\left(v+v_{0}\right)+\cdots
$$

Then

$$
I-s_{0}+\frac{d I}{d \gamma}\left(\gamma-\gamma_{0}\right)+\frac{1}{2} \frac{d^{2} t}{d \psi^{2}}\left(v-\gamma_{0}\right)^{2}+\cdots
$$

ware the essubetion $\left(V-V_{0}\right)=A \sin$ at $<K v_{0}$ is valyd. Then, oropition the harionic term:

$$
\begin{aligned}
& t=\left[t_{0}+\frac{A^{2}}{4}\left(\frac{d^{2} \mathrm{I}}{d N^{2}}\right)_{V_{0}}+\cdots\right] \text { D.C. }
\end{aligned}
$$

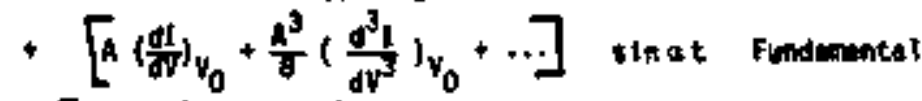

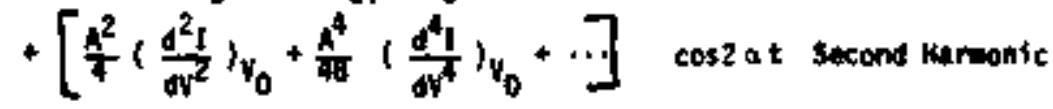

$$
\begin{aligned}
& +\ldots
\end{aligned}
$$

$(E-3-4)$

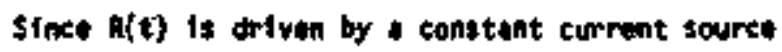

$$
\begin{aligned}
& V(t)=I(t) R(t) \\
& d V=d t A(t)+d R(t) I \\
& \frac{d y}{d I}=R(t)+\frac{d R(t)}{d I} I \\
& \frac{d^{2} y}{d I^{2}}=2 \frac{d R(t)}{d I}+\frac{d^{2} R(t)}{d I^{2}} 1
\end{aligned}
$$

Thus, the complications to aval wate $\mathrm{dR} / \mathrm{dt}$ from the cosfficients of hicher order harnontes in the voltage signsl.

ant, 16. 


\section{Smail Modulation Differentiation*}

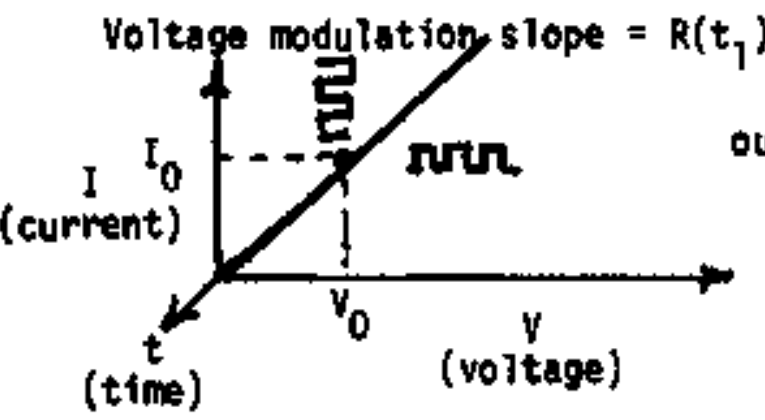

output for detection

Where $R$ is changing in time, and only one value in time is shown. One can imagine later curve on the t the axis for $t=t+\tau / 2$.

1) Assume $R(t)$ is ohmic $\Rightarrow V(t)=I(t) R(t)$.

2) Then the D.C. value of $V(t)$ is proportional to $R(t) \Rightarrow \vec{V}(t)=$ $I_{0} R(t)=V_{0}(t)$ for $I(t)=$ constant and either $0 . C$. or Low Freq.

3) Then the time derivative of $v_{0}(t)$ is $\Rightarrow v_{0}(t)=I_{0} \frac{d R(t)}{d t}$.

4) Thus, by using a high frequency voltage modulation at $\omega(-1 / \tau)$ of $v_{0}(t)$, such that $v_{0}(t) \Rightarrow v_{0}(t) \times\left[\begin{array}{l}\text { SQ wave } \\ \text { of period } \tau\end{array}\right]$.

Then

$$
v_{0}(t)=\frac{\left[v_{0}(t)-v_{0}\left(t+\frac{\tau}{2}\right)\right]}{\tau / 2} \sin 2 \omega t+\cdots
$$

5) Thus the detected value of $V_{0}(t)$ of 2 w detection

$$
\nabla_{0}(t)=\frac{1}{\tau^{\top}} \int_{0}^{\tau^{t}} \frac{\left[v_{0}(t)-v_{0}(t+\tau / 2)\right]}{\tau / 2} d t
$$

and

$$
\bar{V}_{0}(t)_{2 \omega}=\frac{1}{\tau} \int_{0}^{\tau} I_{0} \frac{[R(t)-R(t+\tau / 2)]}{\tau / 2} d t
$$

Then $1 n$ the

$$
\lim _{\tau \rightarrow 0} \bar{v}_{0}(t)_{2 \omega}=\frac{1}{\tau} \int_{0}^{\tau^{2}} I_{0} \frac{d R(t)}{d t} d t=I_{0} \frac{\overline{d R}(t)}{d t}=\bar{v}_{0}(t)
$$

"Ref. 17. 


\section{MULTIPLIER BOX}

PWR.
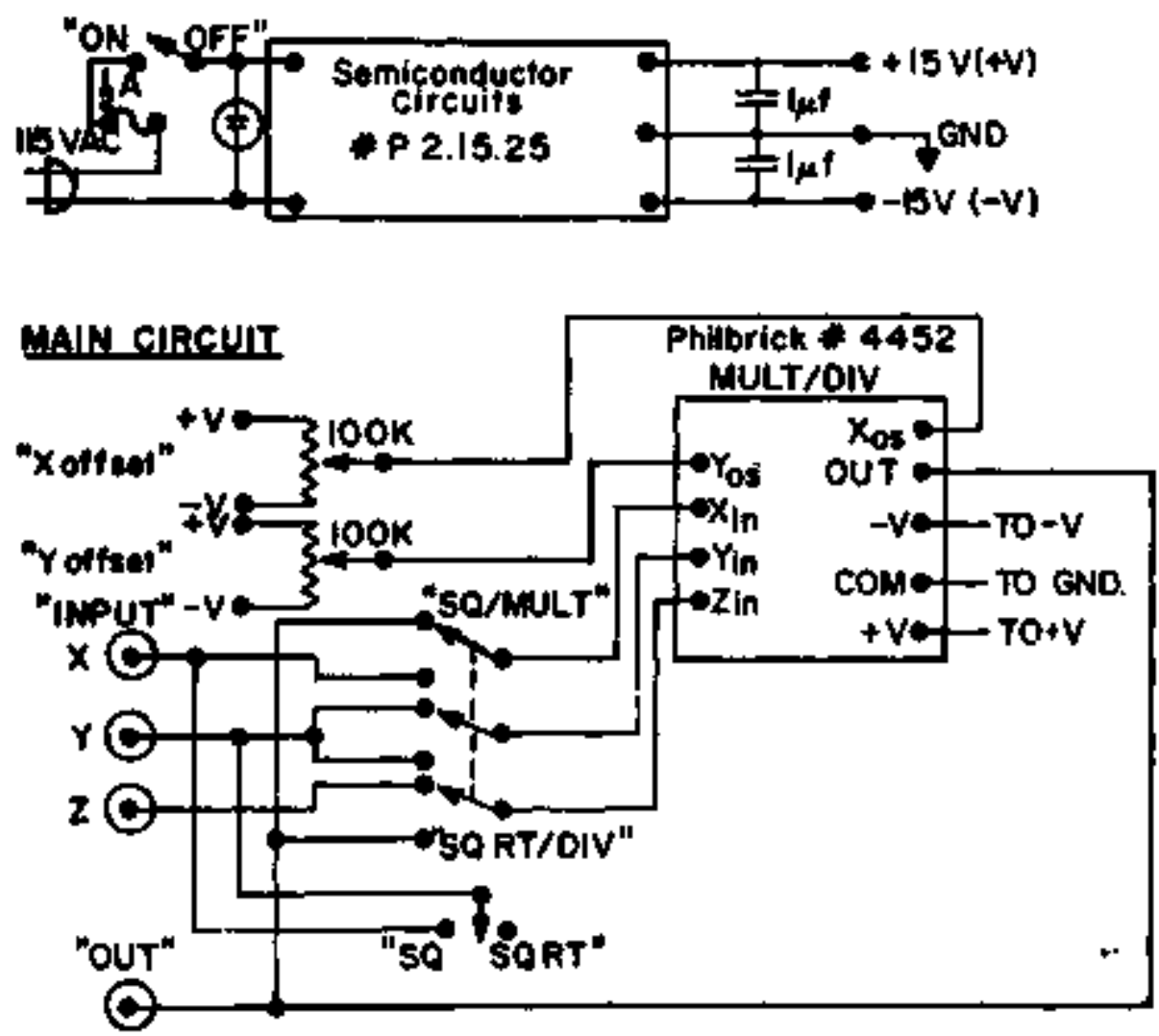

Figure E-5. Calibration and Use.
FOR: MULT ( $\left.\left|e_{x}\right|,\left|e_{y}\right| \leq 10 ; e_{0}=\frac{-e_{y} e_{x}}{10}\right)$ or SQ $\left(\left|e_{x}\right| \leq 10 ; e_{0}=\frac{-\left\langle e_{x}\right)^{2}}{10}\right)$

SET: $x=0, y= \pm 10 \vee 100 \mathrm{~Hz} \quad$ SET: $y=0, x= \pm 10 \vee 100 \mathrm{~Hz}$ $A D$ : $x_{0 S}$ for null out THEN ADJ: $y_{O S}$ for null out

FOR: DIV $\left(e_{z} \leq e_{y}, 0<e_{y} \leq 10,\left|e_{z}\right| \leq 10 ; e_{0}=-10 e_{z} / e_{y}\right.$

SET: $y=+10, z=0$

SET: $z=+10, y=+10$

$A D$ : $x_{0 s}$ for null out THEN $A D S: y_{0 s}$ for -10 vout

FOR: $\quad$ SQRT $\left(-10 \leq e_{z} \leq-1 ; e_{0}=\sqrt{-10 e_{z}}\right)$

SET: $z=-10 \mathrm{v}$

SET: $z=-1 v$

ADJ: $y_{0 S}$ for +10 out THEN

AOJ: $x_{\text {pff for }+3.16 \vee \text { out }}$

NOTE: In divider mode $1 \%$, except for $z=3 \rightarrow 6 \mathrm{v}$, then $5 \%$ error, for $y=10 v$. 
same problem as that of the antlog methods. The problem is that noise is introduced by the frequency of the sampiing, where the more frequent the samples, the higher the amount of white noise that enters into the sampling.

The essential method is to determine from a grid of points sampled from a continuous function, lifne tangent to any point. (i.e., the slope) relative to nearby points. There are many schemes for determining this slope. The inherent problem of this method is that by working with sampled data, there is a noise error in that data associated with the frequency of sarpling. Then the formed function, which involves the ratio of differences ( $\left.\frac{d R}{d T}=\frac{\left(R_{i}-R_{i+1}\right)}{\left(T_{i}-T_{(+1}\right)}\right)$ will be greatly indulated by this noise, especially for fluctuations in the denominator. Blackman and Tukey ${ }^{18}$ present a means of determining a power spectrum for at of digital data. From this spectrum, one cen ossign a digital. fllter that will filter out high frequency fluctuations in the sampled data, and yet retain the major part of the power spectrum. The second consideration was to adjust the sampling methods to be cons istent with the time constants of the system (see Ref. 19, page 82). The sampling rate is such that the time constant of sampling is slightly less than the time constant of the lock-in, but at frequency compatible with the cutoff frequency in the power spectrum, so that higher fluctuations (noise) are reduced from the dato. The filter finally ased on the data (for both $R, T$ ) was of the form: 


$$
f_{i} \cdot\left(f_{i+1}+2 f_{i}+f_{i-1}\right) / 4
$$

followed by,

$$
f_{i}=\left(f_{i}+f_{i+1}+f_{i+2}\right) / 3
$$

Where $f_{i}$ is the value of the function at the $i^{\text {th }}$ point in time. Because the rate of temperature change when the transition is taken is constant ( $\frac{d T}{d t} * r$ ) and since fluctuations in the denominator ( $d T$ of $\frac{d R}{d T}$ ) can increase dramstically the fluctuations of the function ( $\left.\frac{d R}{d T}\right)$, the derivattve was formed by:

$$
\left.\frac{d R}{d T}\right|_{i}=\frac{\left|R_{i+1}-R_{i-1}\right|}{2 \Delta T} \text { where } \Delta T=r t_{\text {total }} / \text { samples }
$$

This was filtered by the above filter (eq. E-5) to give:

$$
\left.\frac{d R}{d T}\right|_{i}=\left(R_{i+3}+R_{i+2}-R_{i}-R_{i-1}\right) /(3 \Delta T)
$$

The resulting function was filtered aga in by both filters (eqs.E-4,E-5) This gave a satisfactorily varying slope that was consistent with al1 the time constants of the system, and thus did not arbitrarity alter the data. The proof of this was that successive applifations of the filters did not alter the results, indicating that fluctuations the below the cutoff frequencies of the filters.

It should be noted that the problem of noise in the andlog 
differentiation schemes is the exact problen of noise associated with the digital schemes. In fact, one can approach the analog problem using fint te differential methods and draw an analogy between spectfic analog ctrcuit, and a specific form of a digital filter. A simple example would be of the form:

$$
\begin{aligned}
& \text { Anatog } \\
& \text { Digital } \\
& v(t) \underset{R}{\infty} v_{c}^{\infty} \frac{L}{T}(t) \\
& V(t)=V_{n}\left(\Delta t_{n}\right) \\
& V^{\prime}(t) \sim \frac{1}{R C} \int V(t) d t \\
& v_{n}\left(\Delta t_{n}\right)=\frac{v_{n}+v_{n-1}+v_{n-2}}{3} \\
& \text { where } R C=3 \Delta t
\end{aligned}
$$

Becouse the data was in digital form to 2 pp $10^{4}$ and the many forms of later analysis could be made avatlable to recorded digital data, the andog schemes for differentiating were discarded. Another possible form of differentiation is to fit the digital data to a polynomial, and then take the analytic differential of that polynomial and generate the dertvative of the original data. This method was not used due to the fnherent errors of differontiating a polynomfal that sometimes has very fastly changing regions. It was believed that one should stay as close to the raw data as possible, and thus, that was why only slight smoothing was incorporated in calculating the derivative, $\frac{d R}{d i}$, of the resistive transition.

The derivative becand a sizeable contribution to the error in 
the experiment, especially with "kinky" resistive transitions. But, by using the digital differentiation method outlined above, this error could be reduced, without distorting the true functional form of the derivative of the resistive transition. 
Appendix F. A.C. Calorimetry and the Heat Conduction Problem

The measurement of samples with small heat capacities has recently been expanded by the A.C. Calorimetry method. The method was first proposed by Sullivan and SeideI, ${ }^{20}$ who used it to measure the superconducting transition of Indium in a magnetic field. The basic idea is that the sample, heater, and thermometer used fn the heat capactity masurenent have a characteristic time constant that is considerably smaller than the time constant of coupling this system to an isothermal bath. Then, one continuously applies heat pulses of length long enough to allow the calorimeter to be in equftibrium, and yet short enough so the isolation link to the isothermal bath does not have time to conduct the heat pulse Figure F-l gives brief description of this idea. A further requirement on the isolation Ifnk, is that ft has to couple the sample well enough to the bath so the D.C. rise in temperature of the 5 ample due to the integration of the A.C. heat pulses is not too large. In this experiment, the sample's $0 . C$. temperature is Increased in time anyway, so that this requirement is not as important.

In analyzing the thermal relations of this experiment, it is quite helpful at times to formulate the problem in such a way as to lend a direct and logy with an equivalent electrical circuit problem. In figure $F-1$, thermal problem involving slab of material with density $p$, thermal conductivity $K$, heat capacity 


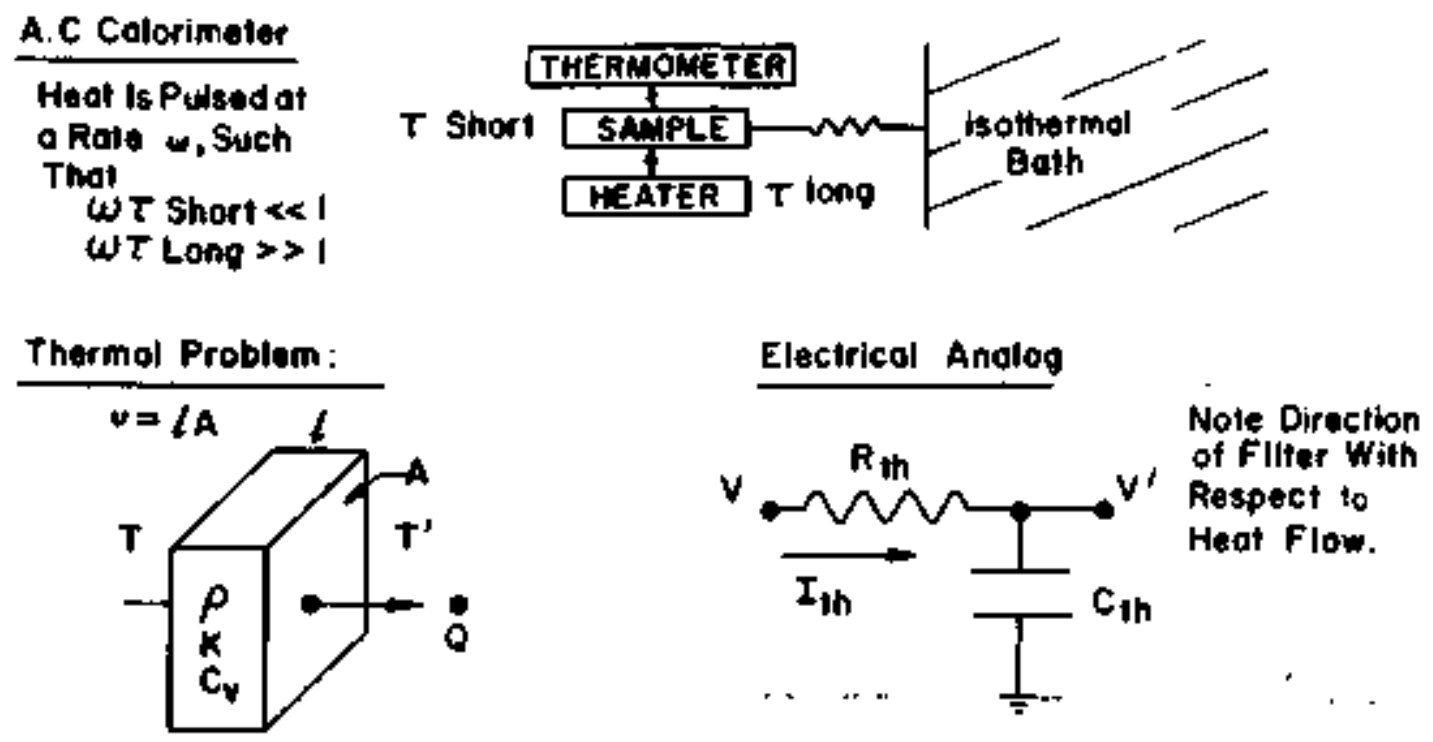

Tomp. Referenced To loolhermal Bath

Voltage Roferented To Eleclitical Gind.

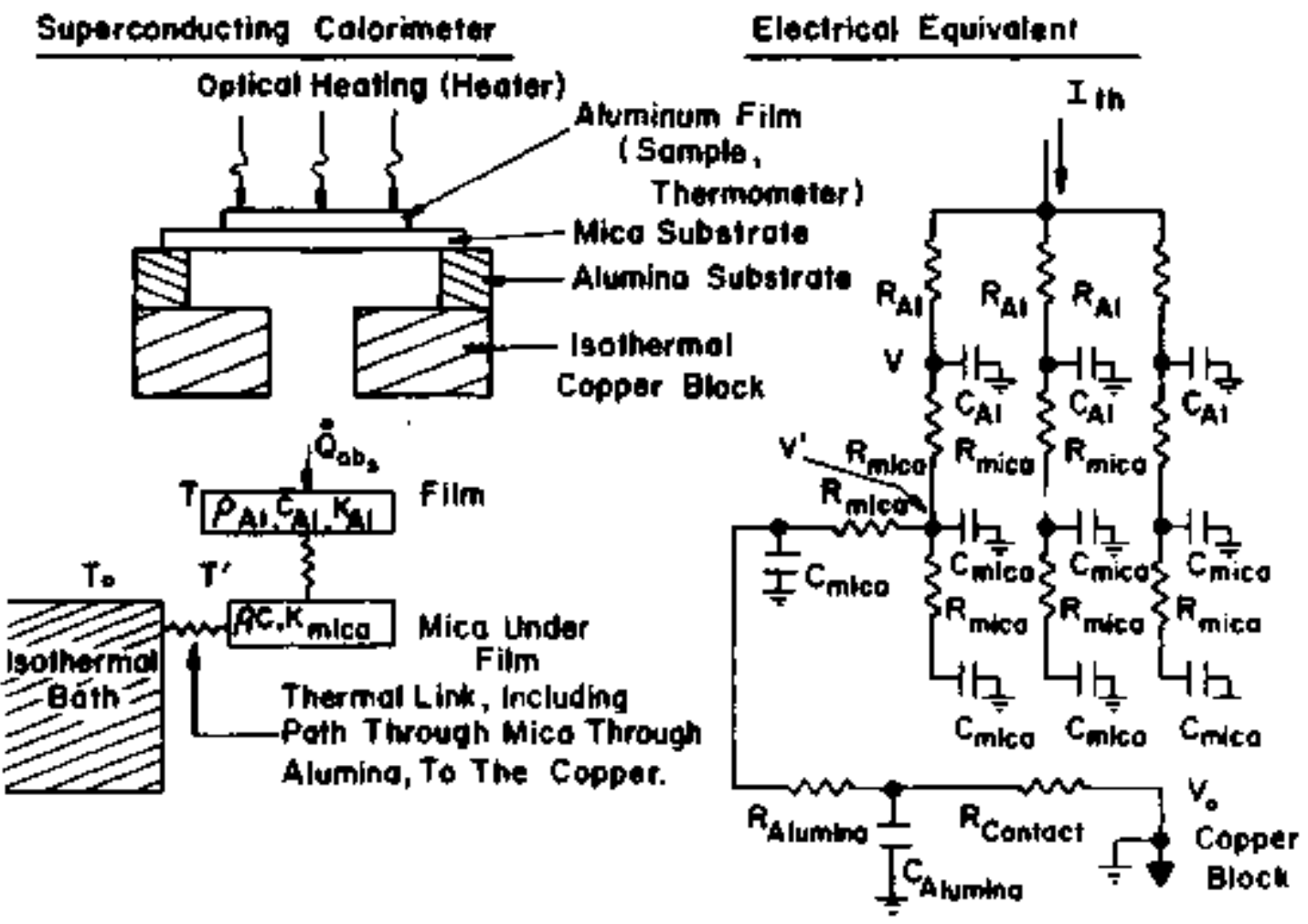

Fi gure $F-1$.

The A.C. Calorimetery method is shown with an equivalent electrical analog. For further details see text and Appendfx $C$. 
per mass $C_{y}$, and length 2 , and ares $A$ is shown (all in C.G.S. units). A power of $\dot{Q}_{a b}$ (j conducted through the slab in the direction shiown and is terminated off at some isothermal bath, such that all temperatures are really temperature differences relative to the bath $\left(T_{0} \times 0\right)$. The electrical analog shown in Ftgure F-1 has an equivalent circuit that involves two temperatures $(V, V)$ a heat flow $\left(I_{t h}\right)$, a heat capacity $\left(C_{t h}\right)$, and a thermal resistance $\left(R_{t h}\right)$. In Table F-1, the analogy is shown in more ' exact detafl, where an internal heat source (in the slab) is included. The lectrical equivalent to this heat source is to have an internal current source (a battery in series with a large resistance, and in parallel with R). The three equations in a differential time dependent thermal probtem are shown first in Table F-1. F is the power flow/area, and "a" is the internal heat source which is equal to an energy density/time. The electrical equations are written in such a way that they have a one to one correspondance to the famfllar themal problem. Integration by parts over a very short path integral is used in deriving the third equation (i.e., $\frac{J}{v} \int_{x}^{x+\Delta x} \frac{\alpha V}{\alpha x} \cdot d A \approx \frac{\alpha^{2} v}{\alpha x^{2}}$ for $\Delta x$ small). I int is the internal current source of the resistor $R$. Once this analggy has been established, a finite difference approach (i.e., $\left.\alpha T+\Delta T, \quad \alpha V+\Delta V, \alpha X_{+}+\ell\right)$ can be used to restate the problem. The second set of equations restate the thermal problem in an electrical form, with appropriate thermal capacitances, resistances, and time constants. The electrical current flow is referred to Bositive current flow in order to obtain the familiar potential 
Table F-1

\section{Electrical Analogy to Heat Flow}

Quantity

Heat

Electric

I. Electrical eqs, in heat form:
1) resistance

$$
F=-k \frac{\partial T}{\partial x}
$$
$\mathrm{J}=-\sigma \frac{\partial V}{\partial x}$
where $F=\dot{Q} / A$
where $\frac{d q}{d t}=\int J \cdot d A$
2) capacitance
$\frac{\partial F}{\partial x} \div \rho c \frac{\partial T}{\partial t}=a$
$\frac{d q}{d t}+C \frac{\partial V}{\partial t}=I_{\text {int }}$

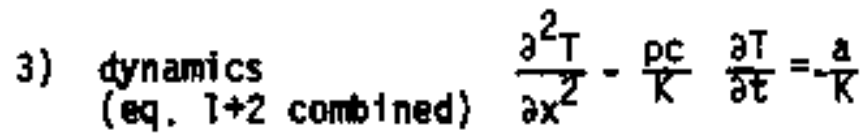
$\frac{\partial^{2} y}{\partial x^{2}}-\frac{C}{\partial v} \frac{\partial V}{\partial t}=\frac{-I_{\text {int }}}{v \sigma}$

11. Heat eqs, in electrical form:

1) thermat resistance $\Delta T=-\frac{d Q}{d t} R_{t h}=-t_{t h} R_{\text {th }} V=-\frac{d q}{d t} R=I R$

$$
\begin{array}{ll}
R_{t h}=\ell / K A=I_{t h}=\dot{Q} & R=\frac{d x}{\sigma A}, I=-\frac{d q}{d t} \\
\Delta T=\left(T-T^{\prime}\right)=-T_{R}, \ell=d x & \Delta V=\left(V-V^{\prime}\right)=V_{R} d x=\ell
\end{array}
$$

2) thermal capacitance $I_{\text {th }}+C_{\text {th }} \frac{d T^{\prime}}{d t^{-}}=I_{Q} \quad \frac{d q}{d t}+C \frac{d V^{\prime}}{d t}=I_{\text {int }}$

$$
C_{t h}=\rho c v, I_{Q} * a v
$$

3) anamics $T_{R}=I_{Q} R_{t h}+t_{t h} \frac{d\left(T_{R}+T\right)}{d t} \quad V_{R}=-I_{\text {tht }} R+T \frac{d\left(V_{R}-V\right)}{d t}$ $\tau_{\text {th }}=\ell^{2} / K=C_{t h} R_{t h}, K=\frac{K}{\rho c}, \begin{aligned} & \text { and } T \text { is always referenced to same } \\ & T_{0} \text { (1 sothernal bath) }\end{aligned}$ $\tau=R C$, and $V$ is always referenced to same $V_{0}$ (voltage grnd.)

and the two systems are connected by power exchange
4) power exchange $P_{\text {th }}=\frac{T_{R}}{R_{\text {th }}}$ (joules) $+P=\frac{V_{R}^{2}}{R}$ (joules) 
differences. Thus, al though equations II-1 differ in appearance (the minus sign), they are the same physical concept. The equations II-3, showing the dynamics, are also equivalent. This is because $\tau$ and $V$ are constants in time, and one refers to positive potentials for negative electrons flowing backwards (the sign in front of $I_{\text {int }}$ ). These equations can be converted back to the differential fom of equations I-3 by using the finite difference substitutions. Note that there is no need for therna; inductances. Equation II-4 of Table F-l relates the power in the two systems. As will be shown in a later appendix, one can interface an electrical circust to thermal probiem, by formulating the thermal probiem in electrical form and equating powers in each system through some thermal constant resistance (heater-thermometer barrier) using equation II-4. Thus, the electrical analogy shown in figure $F-1$ is complete for the thermal problem, in the limit of small thicknesses and for the low pass filter to be oriented in the correct direction relative to the heat flow. The differences in the signs of the analogy of Table $F-1$ is related to the potential drop for positive current in the lectrical problem, and an energy loss for the hoat flow in the thermal problem.

Originally, an attempt was made to solve the problen by normal anatytical methods using the therma 1 equations of Table F-1, part $I$. The problem was formulated as that of a $1000 \&$ atuminum $\mathrm{f1} 1 \mathrm{~m}$, heated by optical pulses $(6328 \&)$, in contact with a $50,000 \&$ alumina substrate in contact with an isothermal copper block. 
Since the attenuation constant of the 11 ght in the 8 luminum is about $6 \times 10^{5} \mathrm{~cm}^{-1}$, 21 almost no light is transfilted by the film, and since $92 \%$ is reflected, about $8 \%$ of the incident radiation is absorbed by the film. This compares with transmission measurements of the films which show $\sim .1 \%$ transatission. This reduces the problem to that of a source ariving the film throughout, and since the film's thermal time constant ( $\tau_{\text {th }}=\frac{\ell^{2}}{K} \sim 10^{-13}$ sec.) is much smaller than the driving frequencies, we can assume it to be isothermal.

The problem in the analytic solution is in matching the boundary conditions at both the vacuum interface (on the exposed aluminum side) and on the aluminum-alumina interface. An attempt was made to solve the problem by the mathod of fint te differences, where essentially the differential equations of Table F-1 are transforred to fintte differences amounts. The one spatial dimension is divided into swall inerenents $o x=h$, and time is divided into increments $\Delta t=r h^{2}$, which are normalized by the medium's themal diffusivity constant ( $\Delta t=\Delta t_{\text {real }} k_{\text {medium }}$ such that:

$$
\begin{array}{r}
\frac{\partial^{2} T}{\partial x}=\frac{\partial T}{\partial t}+T_{j, k}=T(j \Delta x, k \Delta t) \text { being the temperature } \\
\text { at the } j^{\text {th }}, k^{\text {th increments. }}
\end{array}
$$

Equation ( $F-T$ ) is then: 


$$
\begin{aligned}
& T_{j, k+1}-r \theta\left(T_{j=1, k+1}-2 T_{j, k+1}+T_{j+1, k+1}\right)=T_{j, k}+r(1-\theta) \\
& x\left(T_{j-1, k}-2 T_{j, k}+T_{j+1, k}\right) \text { where } \theta \text { is an arbitrary parameter, } \\
& \text { and } r=\Delta t /(\Delta x)^{2}
\end{aligned}
$$

As pointed out by Crandal1, for different values of $\theta$, we have different forms of the finite differences equations. The two most well known are the Implicit $(\theta=1)$ and the Explicit $\{\theta=0)$ and possibly the crank and Hicolson form $\left(\theta=y_{2}\right)$. The problem with the explict forts is that $r\left(* \Delta t /(\Delta x)^{2}\right)$ has to be less than $1\left(\sim w^{2}\right)$, or otherwise unstable, oscillating solutions result. This constraint is equivalent to the heat flow reversing direction between difference evaluations. Cranda $11^{22}$ varied $r$ and $\theta$ in an arbitrary way to map out the solutions. One can get stable solutions as in the thods mentioned before, but the accuractes are not to orders higher than $\theta\left(h^{4}\right)$. By choosing $r=0.2235, \theta=0.1273$, he found stable solutions to an accuracy of $\theta\left(h^{6}\right)$. The boundary condition imposed on the problem is that $\Delta t_{1}=\Delta t_{2}$, for the two media (aluminum, alumina). Since $r<$ implies $\frac{\Delta t_{\text {real }} k^{2}}{(\Delta x)^{2}} \leq 1$, thts sets a constraint on the maximum size of time 1ncrement: And $\Delta t_{1}=\Delta t_{2}$ sets a size on the grid ratios of the two medi uns (1.e. $\Delta x_{1}=\sqrt{\frac{k_{1}}{k_{2}}} \Delta x_{2}$ ). Essentially one nomslizes. the grids so the problem looks like one medflom. But since $\sqrt{\frac{K_{\text {Alumina }}}{\text { KAlumi num }_{\text {A }}}} \approx 10^{-2}$, we need $100, \Delta x_{\text {aluwi num }}$ increments to have 
Just one $\Delta x_{a}$ umiga. And, since only two $\Delta x_{a l}$ lumina means $\Delta x_{\text {a Iuminum }}=\frac{1000 \mathrm{~A}}{2 \times 10^{2}}=5 \times 10^{-8} \mathrm{~cm}$, our stability requi rentent means that our maximum time increnant is:

$$
\Delta t=\frac{(\Delta x)_{\text {aluninum }}^{2}}{k_{a} \text { luminum }}=\frac{\left(5 \times 10^{-8}\right)^{2}}{10^{4}} \sim 10^{-20} \text { sec. }
$$

One can see that a computer solution is going to be quite costly to monitor even sust one second of time in the problem. The basic nature of the problem, that of a very thin, fast thermal material, in contact with aery thick, slow themal material, means that one has to have many increments in space to normalize the sizes, and many increments in time to nomalize the thermal responses.

One can take the problem directly over to an electrical anaiog probiem and try to do an and log solution with an equivalent electric circuit. But, the same constraints are there, and one is talking about hundreds of $R, C$ networks.

The problen: was attempted on a 6600 in varying degrees of approximations, but was not concluded due to the large computer times involved and the staplification arguments presented later. Most graphical solutions ${ }^{23}$ are not useful since they are not in the ranges needed.

A better way to analyze the problen is to assume the aluminum is an isothermal slab, following the oscillations of the optical heating. This is justified since the light is absorbed throughout the $f 11 \mathrm{~m}$, and the film has a tfure constant very much smaller than the period of heating $\left(10^{-3}+10^{-4} \mathrm{sec}\right)$. Thus, if one is heating 
a self-supported film, without any substrate, the heat capacity would be:

$$
c_{f+1 m}=\frac{\Delta Q}{\Delta T}=\frac{\dot{Q}_{\text {abs. }}{ }_{\text {osc }}}{\Delta T_{\text {measured }}}
$$

If the film was attached to a substrate, 1tke the problem of an aluminum film on an alumine substrate, the heat capact ty would be different. Now an amount of the substrate would follow the film's temperature oscf1lations, and thus reduce the aT measured. This amount is just the area (A) of the substrate under the film times the thermal langth. where $l_{\text {themal }}=\sqrt{\tau_{\text {osc }} \kappa_{\text {sub }}}$. Then the heat capacity of equation ( $F-3)$ becomes:

$$
C_{f 1\} m}=\frac{\dot{Q}_{\text {abs. osc }}{ }^{T}}{\Delta T_{\text {measured }}}-C_{\text {sub }} A \sqrt{\tau_{\text {osc }} \kappa_{\text {sub }}}-C_{\text {other }} A \sqrt{\tau_{\text {osc }}{ }^{k} \text { other }}
$$

where the other contribution could be from an adsorbed He film. Equation (F-4) is just a ist order approximation to the problem. A wore complete description would be related to the temperature pulses measured ( $\Delta r$ ) for the heating pulses applied. By constructing a number of low pass ftiters in series, one can approximate the problem. Thus, by going to highar frequencies in heating, one has a shorter time constant associated with the temperature oscillations, and equation (F-4) becomes (F-3). This is the sape as saying the low pass filters become larger impedances to the high frequency heat pulses, and thus do not attenuate the temperature oscillations (AT). Figure F-2 shows such behavior for an maiog solution to a 


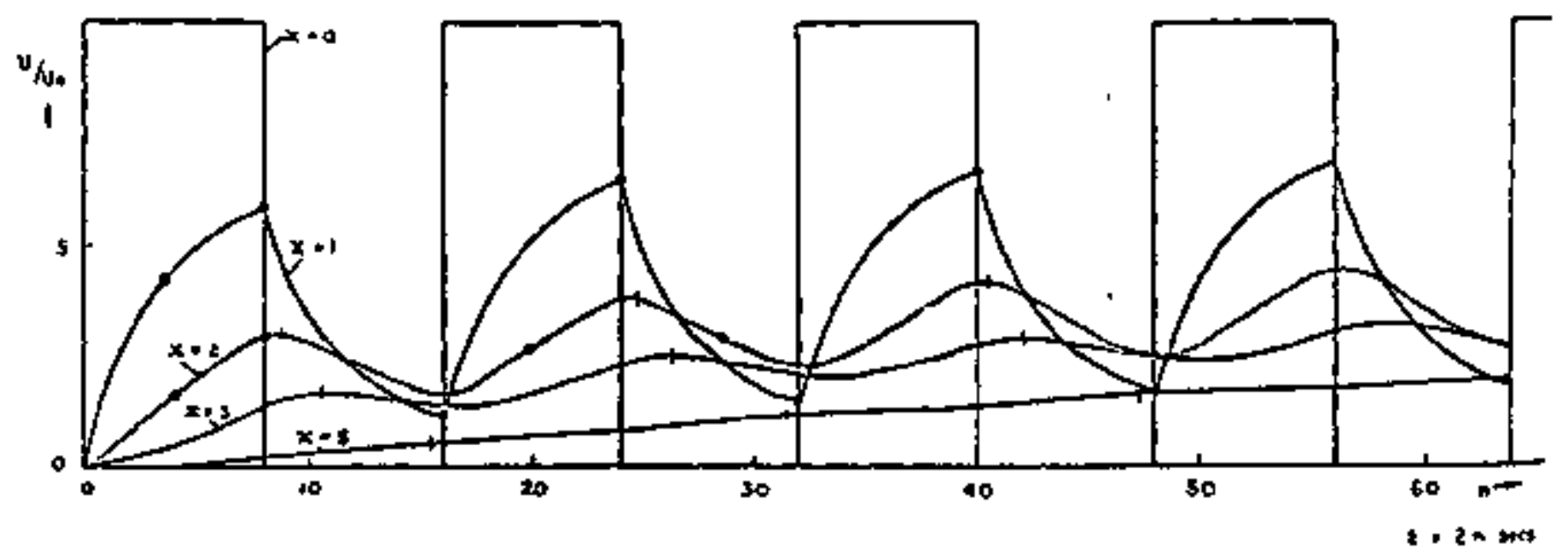

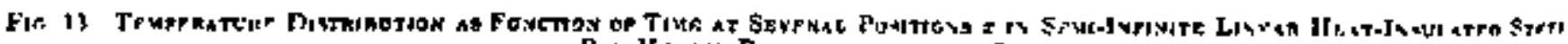

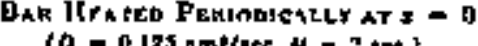

Figure $\mathrm{F}=2$.

The temperature profile for an analog solution of the heating of a stae) bar by a step temperature pulse one end $(x=0)$ monitored at successive points down the bar $(x=1,2,3,5)$ as a function of time. Note that after a short time a steady state $\mathbf{1 t u a t i o n}$ results, with only the first few monitor points following the temperature pulses. (from G. Liebmann, Trans. Of ASME, 78-1, 655, (1956)). 
heated steel rod. Figure F-l shows the transformstion of the discrete thermal problem in the experiment to the equivalent, Ist order electrical circutt. This circuit is only a guide, and is in no way the exact equivalent, since the constraints on finite difference grid have not been applied. To determine the thermal time constants of the materials, one needs $p, K, C$. It should be noted that even if $C$ or $K$ for the film were diverging quantities, they still could never change the films' time constant.

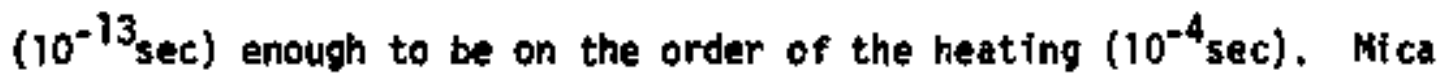
was chosen as a substrate because of its layered nature. Operating at 1.5K, wight produce a rather large Kapitza boundary resfstance between the layers and thus give it a very small effective transverse thermal conductivity $(K)$. Films evaporated on the mica substrates did not at first have an A.C. heating signal. At first this problem was thought to be related to the film being too closely coupled to the substrate, and thus it could not heat up at all. Later, it turned out to be problem in the electronics. But, because of the initial failures, self-supported fflms were made and tested. These films could be optically heated. Since by this time the electronics problem was solved, films on mica and glass were also tried. They both give optical heating. (up to $20 \mathrm{KHz}$ ) although the signals were not as large for the filats on glass. A rough comparison is given below: 


\begin{tabular}{|c|c|c|c|c|c|}
\hline Run * & Substrate & $\Delta \mathrm{T}$ & Q(approx.) & $\underline{T}$ & $\underline{\mathrm{C}}$ \\
\hline $30 I$ & mica & $7 \times 10^{-6} \mathrm{~K}$ & $2 \times 10^{-12} w$ & $10^{-3} \sec$ & $2.85 \times 10^{-10} \mathrm{~J} / \mathrm{k}$ \\
\hline 32 & glass & $4 \times 10^{-7} \mathrm{~K}$ & $10^{-12} w$ & $10^{-3} \mathrm{sec}$ & $2.5 \times 10^{-9} \mathrm{~J} / \mathrm{K}$ \\
\hline 260 & se]f-support. & $1 \times 10^{-5} K$ & $2 \times 10^{-11} \mathrm{~W}$ & $10^{-3} \mathrm{sec}$ & $1 \times 10^{-9} \mathrm{~J} / \mathrm{K}$ \\
\hline
\end{tabular}

The film on glass is measuring a larger heat capacity for roughly the same film as the film on mica. This would indicate that the f11m on the glass substrate was closer coupled to the substrate than the film on mica. The self-supported film had a different light source, and so it quite possibly is an inaccurate comparison to the other two filins. As an independent check on the coupling of films on mica, measurenent of the transverse diffusivity of nica was wade. A manganin heater was evaporated on the back side of a film $(100 \Omega$ and same area as film) on a piece of mica. In thts way, heat pulses could be created on one side of the $\sim .2 \mathrm{mi}$ l. thick piece of mica, and detected on the other side by the superconducting film. The geometry of the filus was verified by measuring the capacitance of the two films with mica as the di-lectric. The heater was driven with a voltage biased heating power at a frequency of $\omega$, and the film was monitored for heating at both $2_{\omega}$, and $\omega$ (indicating just electrical coupring). From frequencles as low as $2 \mathrm{~Hz}$ up to $10 \mathrm{KHz}$, no A.C. heating putse could be detected, that was not fust an electrical coupling of the voltage at w detected at $2 w$. By knowing the power of the heating pulses and the sensitivity of the detector, lower limit on the 
heat capact ty of the mica could be determined. Thus the heat capacity of the mica between the film and the heater (area $\sim 1.2 \mathrm{~cm}^{2}$, thickness $\sim 5 \times 10^{-4} \mathrm{~cm}$ ) could be estimated as being greater than $10^{-4} \mathrm{~J} / \mathrm{K}$ (1KHZ measurements) $+10^{-3} \mathrm{~J} / \mathrm{K}(2 \mathrm{~Hz}$ measurements), which does not compare to a calculated value of $\mathrm{C} \sim 8 \times 10^{-8} \mathrm{~J} / \mathrm{K}$ using the data of table F-2. But, this is assuming a steady state, and if the transverse thermal conductivity was very small, then the time constant of the heat pulses was too short for the equilibrium time between the heater and the film. A diffusivity measurement was also made of the mica. The heater was driven with a step pulse, and the temperature of the film was monitored. For 1arge pulses of the heater, the film would rise with an exponential time constant of 1 sec to a higher level, It took $2.5 \mu \mathrm{W}$ to raise the film.7mk over a time of $1 \mathrm{sec}$. For the thickness of the mica and a thermal time constant of $1 \mathrm{sec}$, this gives a diffusivity of $2.5 \times 10^{-7} \mathrm{~cm}^{2} / \mathrm{sec}$ for power of $2.5 \mu \mathrm{w}$. Since the heat capacity and the density are somewhat better tnown (or estimated, see Table F-2) for mica, this would indicate a thermal conductivity of $\mathrm{K} \sim 2.85 \times 10^{-11} \mathrm{~W} / \mathrm{mk}$ at 2K. This would also indicate why A.C. heating at $2 \mathrm{~Hz}$ did not produce thermal steady state conditions. It should be mentioned, that if the data for the pulse testing is used to calculate an equivaient hat capacity to absorb the heat within the time constant measured, one finds an equivalent heat eapacity of $C_{\text {equiv }} \sim 3 \times 10^{-3} \mathrm{~J} / \mathrm{K}$ (where $C_{\text {equiv }}=\frac{d_{\tau} \tau}{d T}$ ), which agreas with the levels set by the A.C. heating measurements. Thus from these pusse and A.C. heating 
Table F-2, Theranl Properties at Low Temporatures of Naterials Lsed.

\begin{tabular}{|c|c|c|c|c|c|}
\hline Material & Therall conduct ance (k) & Density $\{0\}$ & Heat Capacity (C) & $\left\{\begin{array}{l}\text { Diffusivity }(k) \\
\text { Culeulated } \alpha=K \text { K } c\}\end{array}\right.$ & $\left.{ }_{\text {Coments }}=z_{/ m}\right)$ \\
\hline Mica & 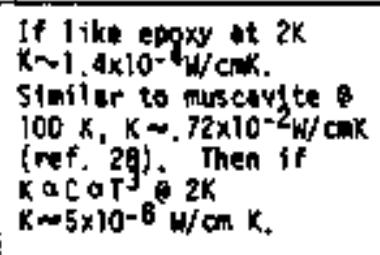 & 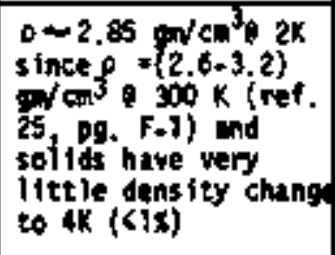 & 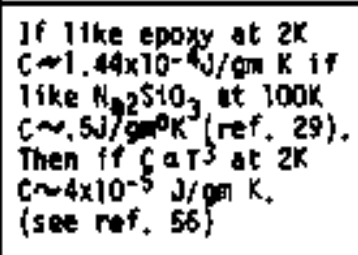 & 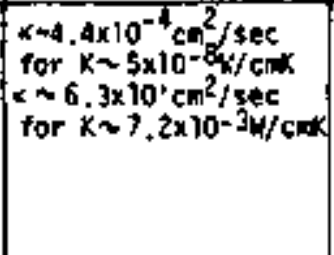 & 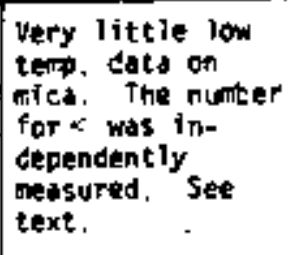 \\
\hline $\begin{array}{l}\text { Glass } \\
\text { (Dyrex) }\end{array}$ & $\begin{array}{l}\text { gik } k+1.2 \times 10^{-4} w / \mathrm{cm}^{k} \\
\text { (ref. } 24 . \mathrm{pg} .30\}\end{array}$ & 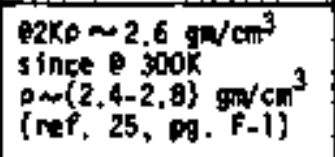 & $\begin{array}{l}\text { ofk } c \sim 2.4 \times 10^{-1} \mathrm{j} / \mathrm{gm} x \\
\text { (ref, } 25, \mathrm{pg} .13 \mathrm{~g})\end{array}$ & $\cos _{2 \mathrm{~K}} \sim 1,9 \mathrm{~cm}^{2} / \sec \theta$ & \\
\hline $\begin{array}{l}\text { Aluminum } \\
\text { (dirty }\}\end{array}$ & $\begin{array}{l}\text { zk } k \sim 4.8 \times 10^{-1} \mathrm{w} / \mathrm{ark} \\
\text { (ref. } 26,09.876)\end{array}$ & 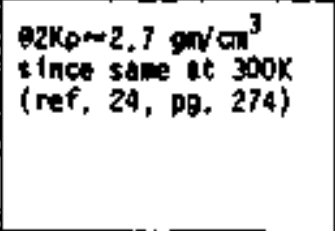 & 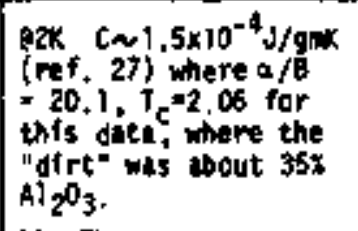 & $\mathrm{c}_{2 \mathrm{~K}}-1.2 \times 10^{3} \mathrm{~cm}^{2} / \mathrm{sec}$ & 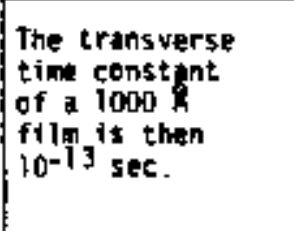 \\
\hline $\begin{array}{l}\mathrm{He} \\
\text { (nonoluyer) } \\
\text { on topper }\end{array}$ & 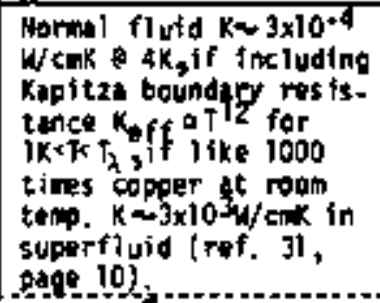 & 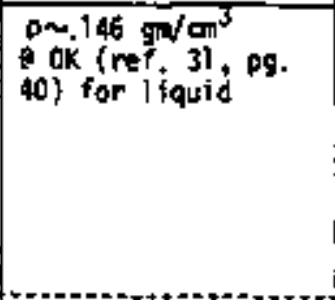 & $($ tref. 3 . & 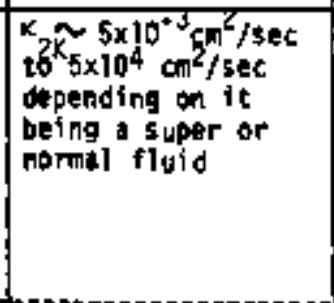 & $\begin{array}{l}\text { The monolayer } \\
\text { may not be o } \\
\text { superflufd and } \\
\text { also the kipitzo } \\
\text { boundary ro- } \\
\text { sistonce will } \\
\text { also reduct } \mathrm{K} \text {. }\end{array}$ \\
\hline Gas & $\begin{array}{l}k \sim 4 \times 10^{-5} w / c m K \quad g 2 K \\
(r \in f .31,99.98)\end{array}$ & 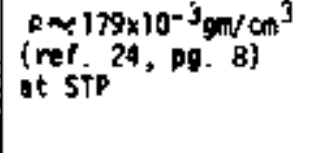 & $\begin{array}{l}\text { C } 5 j / g-k \text { at STP } \\
\text { oc varies linearly } \\
\text { with prossure }\end{array}$ & 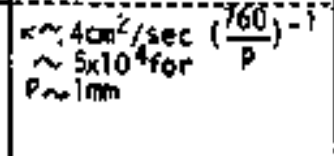 & $\begin{array}{l}\text { He gas at low } \\
\text { pressures has } \\
\text { very sadil } \\
\text { diffusivity }\end{array}$ \\
\hline
\end{tabular}


measurements, it can be assumed that at $2 \mathrm{~Hz}$, a significantly smaller thernal penetration length than $5 \times 10^{-4} \mathrm{~cm}$ exists, and that the themel atffusivity for the nica at $2 \mathrm{~K}$ is $\mathrm{K} \sim 2.5 \times 10^{-7} \mathrm{~cm}^{2} / \mathrm{sec}$.

To completely understand the thermal nature of the experiment, a final measureatent of the 0.C. thermal conductivity of mica was needed. A $\mathrm{f} f 1 \mathrm{~m}$ was evaporated onto a mica substrate $\left(5.08 \times 10^{-3} \mathrm{Cm}\right.$ thick) and the milca was well bonded (Eastman 910 glue) to an isothermal copper biock. The film was biased with an A.C. voltage to determine $\mathrm{its}$ resistance, and 0.C. current to create a power f10w through the mica and thus cause a temperature gradfent. Measurements were made for dffferent powar levels at two points in the film's transition. He gas was adritted and the film's reststance was calibrated to the temperature of the isothermal block. An attempt was made to determine the power of the Taser light absorbed by the firm by masuring the conductivity with it on and off. This measurement put an upper level on the power of $\sim 10^{-72} \mathrm{~W}$ (this is discussed in another section). The results of the transverse themal conductivity were quite revealing. They showed an exponential behavior of the conductivfty vs the power for both sets of measurements for powers greater than $2 \times 10^{-11} \mathrm{~W}$. It had the form shown below.

$$
K_{m i c a} \sim 8 \times 10^{-14} \text { watts } / c m \frac{e^{6.6 \times 10^{2} \Delta T}}{\Delta T}
$$

If no power is dissipated in the f11m, this would give a $\Delta T$ of zero relative to the copper block and give a meaningiess therma ? 
conductivity. Since there is always power dissipated in the film due to the measurements taken, this would limit the $\Delta T$ to be greater than $10^{-3} \mathrm{~K}$. This would give a thermal conductivity of $K \sim 1.5 \times 10^{-10} \mathrm{~W} / \mathrm{cmK}$. One aight expect an exponential form for small temperature differences because of the layered nature of mica. One wight associate a threshold with each Tayer (which can be quite thin, 1.e., a few atomic layers) and each successive layer involves the reduced power in a new threshold, and thus a total conductivity is like a series of resistors going $R, R^{2}, R^{3}$, etc. The thermal conductivity measured in this way agrees reasonably well with the thermal conductivity calculated from the diffusivity. Thus, it is quite reasonable to expect a film on mica to be quite decoupled from the substrate.

Now that the values for the materials of interest are reasonably well known, one can calculate the measured heat capacity at any frequency using rable f-2. It will be:

$$
c_{\text {total }}=\sqrt[A]{T}\left(i_{i} \rho_{i} C_{i} \sqrt{\bar{k}_{i}}\right)
$$

where $A$ is the ared and $1 / \tau$ the heating frequancy. For the materials in the calorfmeter (neglecting He, since it is only adnitted after the measurements), this becomes:

$$
c_{\text {total }}=A \sqrt{\tau}\left\{\quad \rho_{A L} C_{A L} \sqrt{K_{A l}}+\rho_{\text {mi ca }} C_{\text {mica }} \sqrt{\kappa_{\text {mica }}}\right\}
$$


Using the numbers for these materials:

$$
c_{\text {total }}=A \sqrt{\tau}\left\{1.4 \times 10^{-2}+3.7 \times 10^{-7}\right\} \mathrm{J} / \mathrm{Kcm}^{2} \sec ^{\frac{1}{2}}
$$

This implies that if the aluminum was always thick enough, the total heat capacity at any frequency would always be the $\mathrm{ft} / \mathrm{m}$. But, for a thin slab of aluanfum, one has to compare the sizes of the masured heat capacities thenselves. For $1 / \tau=1 \mathrm{KHz}$ they are:

$$
\begin{aligned}
& C_{f i 1 a}=4.8 \times 10^{-9} \mathrm{~J} / \mathrm{K} \\
& C_{\text {mica }}=2.0 \times 10^{-9} \mathrm{~J} / \mathrm{K} \text { for } k_{\text {thermat }}=1.5 \times 10^{-5} \mathrm{~cm}=\sqrt{K \tau}
\end{aligned}
$$

Early measurements were actually made with a slight background of He gas $(P \ll 1 \mathrm{~nm})$. This background only slightly affected the A.C. signal strength, while greatly affecting the O.C. themal conduction. This would imply that the dfffusivity of the He was very small. In view of the $K_{\text {eff }}$ mentioned in Table $F-2$, this could quite possibly be the case.

The fact that an A.C. heating pulse is measured for films on mica, and is conaiderably larger than for films on glass, implies that the aluminum films are quite decoupled from their mica substrate. At nost (for $l / \tau=f K H z$ ) the mica is only $s$ of the total heat capacity measured. If one considers the dranatic possibilities 
that the Kapitza boundary resistance might have in influencing the measurements, it is quite possible that an even smaller percentage of the total heat capactity is the mica. A1so, the heat conduction paths in general are those shown in the equivalent circuit in Figure F-1. This means that less heat is conducted dom into the wica (because it is not terminated) and more is conducted out to the edges. This implies that the films are actually decoupled at high frequencies from the substrate, and appear as if thay were self-supported.

The conclusions presented above make it possible to try to use a superconducting film as a bolometer, if the light is chopped at a h1gh enough frequency. Martin and Bloor 32 made a boloneter that is very smlliar to the apparatus used in this experiment. Their films were on a mica substrate, which was supported by nylon threads whlch had lead leads evaporated on them. The inconting light was chopped at $10 \mathrm{~Hz}$, and the film's resistance was monitored wf th an A.C. Bridge. It is interesting to note that the ir films (Sn) had broadened transitions, and their measurements of the thenmal conductance (including the lead and nylon leads) also had an exponential form. In general, superconducting bolometers are only useful for high frequencies, where a bolometer with fast response is needed. Rose and Bertin ${ }^{33}$ have measured 20 nsec. pulses with a superconducting bolometer. Their model for the equivalent circuit of a bolometer however is in disagrement with that mentioned in the main body of the thesis. They describe two 
modes of a bolometer operation. The first is just a thermal mode, and the second involves a sort of pair breaking scheme taking place in the superconductor. Comparing the superconducting bolometers to other bolometers, they find decreasing tinear relationship between the $\log$ of the sensitivity and the log of the time response of the bolometers. Superconducting bolometers do not have the ultimate sensitivity of other boloneters (doped Ge chips), but do have a faster response time. Th1s increased response time may not be needed in I.R. Astronomy, but areas like Laser Physics might find a use for fast bolometers.

The question of possible pair breaking mechanism taking place in the films used in this experiment is a serious one. If this was the case, the optical heating signal would not be a weasuremant of the heat capactity, but rather measurement of the disturbance of the pair filetd in the superconductor. Testardi 34 and Parker and Wf 11 iars 35 have shown disturbance of the pair field by optical radiation where hv $>A_{\text {gap }}$. Owen and Scalapino ${ }^{36}$. explatn this by a disturbance in the pair field. Since the measurements in this experiment are always for $R>0$, the superconducting system is essentially in futl quasi-particie state, with very few patred electrons. Then, there is no pair field to disturb with the laser radiation, and one has fust a normal metal model for laser radiation absorption. This is confinued by the fact that the resistive transition of the fitms is undisturbed in form with the laser on or off. Thus, pair breaking mechanisms 
are not responsible for the A.C. heating measurements.

In conclusion, the A.C. calorimetry method outlined in this section is being used to measure the heat capact ty of the f11ms. Because of the cholce of mics substrates, and the frequencies of heating used, the films behave as if they were self-supported, and the heat capactty measured can be predominately related to the f11m. This approach also has applications for use as fast bolometers. The thermal heating processes do not involve pair breaking mechanisals, so that one is truly measuring the heat capacity of the f1lms. In this way one can measure the extremely small heat capacities $\left(\sim 10^{-9} \mathrm{~J} / \mathrm{K}\right)$ quite well. 
Appendix G. Analog Servo System.

The experiment involved looking for effects over a temperature interval that corresponded to the critical region of the film. The width of this region was hard to calculate. but it was on the order of $10^{-6} \mathrm{~K}$. This meant that the temperature of the sample would have to be maintained to that degree of accuracy. There are two posstble ways of achieving high thermal stablitity. The first is to have the sample closely tied to very large heat capacity like a bath of $\mathrm{He}^{4}$. thus causing an integrating eqfect on any small thermal fiuctuations. This method was not used because of space available. A second method is to use a heatersensor feedback loop to electronically regulate the temperature of the sample black. Because of the wide success of this method in genoral, it was chosen as the means for reaching high thermal stability. But, bocause of the high accuracy needed, it was. dectded to handle the servo problem in a much more sophisticated manner than the usual crude means of driving a heater with just the off null of an A.C. brtidge reading the teliperature sensor.

The first step was to transform the thermal problem of the relationship between the cryostat, the heaters, and the temperature sensors finto an electricat andog along the lines mentioned in an earlier appendix. This was interfaced to the electrical feadback circuit by the refationship between power in the two systons, 1.e.: 

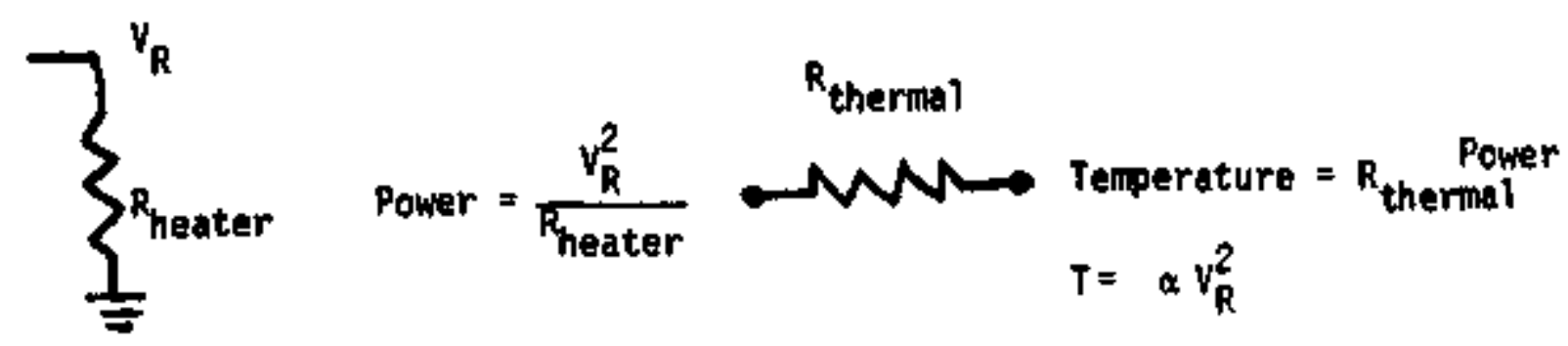

Where $a=\frac{R_{\text {thenwal }}}{\text { Reater }}$

Because of the cryostat's construction' wi th a rather large inner can Isolating the semple block from the $\mathrm{He}^{4}$ bath, it was dectded to sense the temperature errors on the sample block and drive a local heater on the block in a Inear fashion (fine heater), and drivo the inaer can heater (coarse heater) In a non-linear fashion from the same error, but only if the local heater could not handle the error correction.

A first approximation to the overall feedback circuit (including both the thermal and the electricat systens) is shown below (refer to cryostat section for thermal layout):

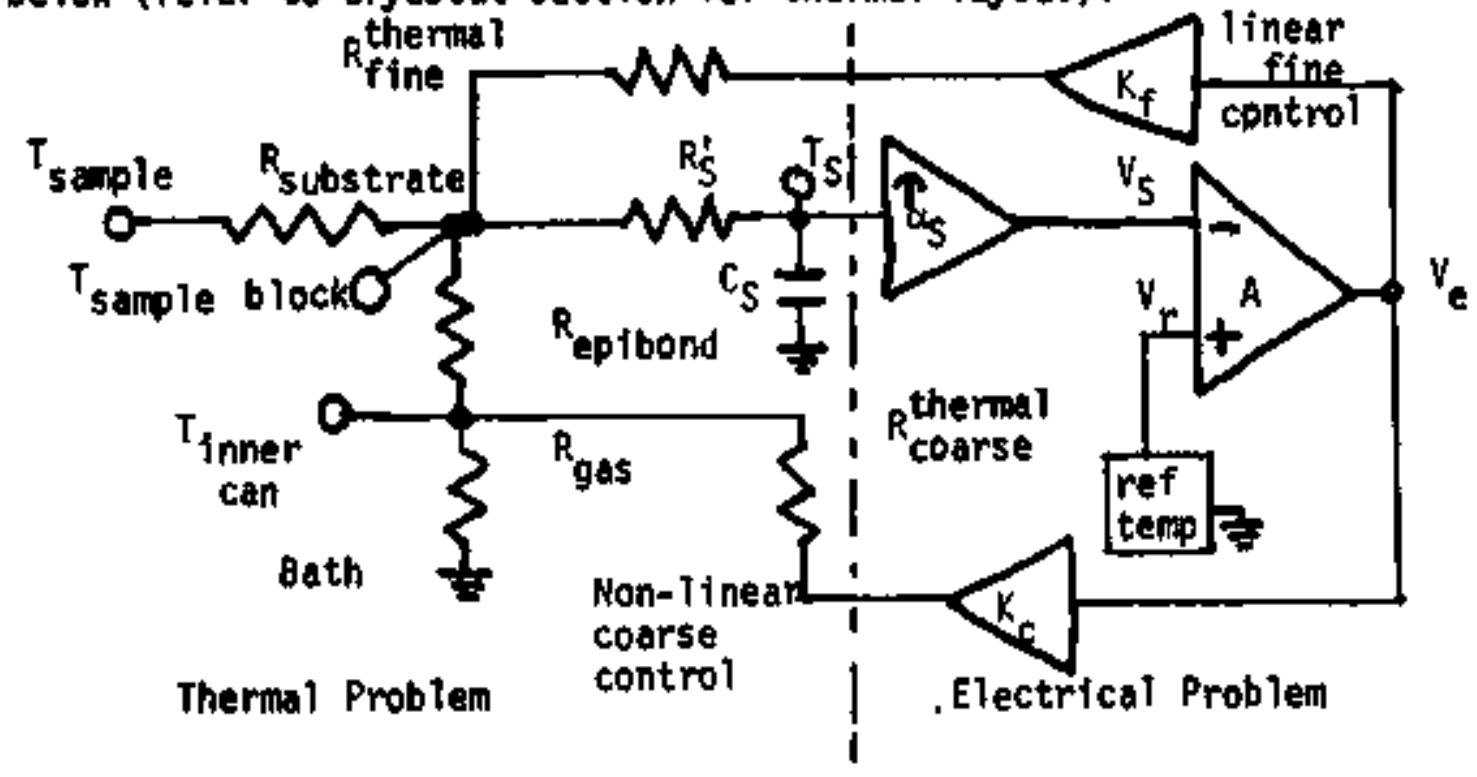


The error valtage $\left(V_{S}\right)$ corresponds to temperature error $\left(T_{s}\right)$ as sensed by the sensor in the sample block. They are related by the gain of the lock-in of the A.C. bridge, and the change in voltage (resistance) of the sensor for a unft change in temperature. This is lupod into the parameter $\alpha_{s}$. This error voltage is added to the reference temperature at which the system is desired to be at and then drives both the 11 near heater control and the non-linear heater control. These two paths of power flow into the thermal problem through their appropriate thermal impedances and complete the feedback loop. The restrfetions on this feedback circuit are:

1) Stable operation--10op gain $\geq 20 \mathrm{db}$ /decade (12 db/octave) $\begin{gathered}\text { electrical } \\ \text { system }\end{gathered}=\tau$ where $1_{\text {sec }} \geq \tau \geq 10^{-2} \sec$

2) For the sensor not seeing thermal
fluctuations of the inner can

3) For true feedback (high open loop gain) $10 \alpha_{f} A_{1} \alpha_{s} \gg 1$

The electrical efrcuit was to produce a function that re]ated the error voltage $\left(V_{s}\right)$ to the correction voltage $\left(V_{e}\right)$ by the graph of Figure 6-I. The correction voltages are related to the correction temperature by:

$$
T_{\text {corrected }}^{\text {course }}=a_{c} v_{e}^{c} \quad T_{\text {corrected }}^{\text {ffne }}=a_{f} v_{e}^{f}
$$

An analog computer was constructed that provided the retationships graphed as well as a wide range of the parameters and 


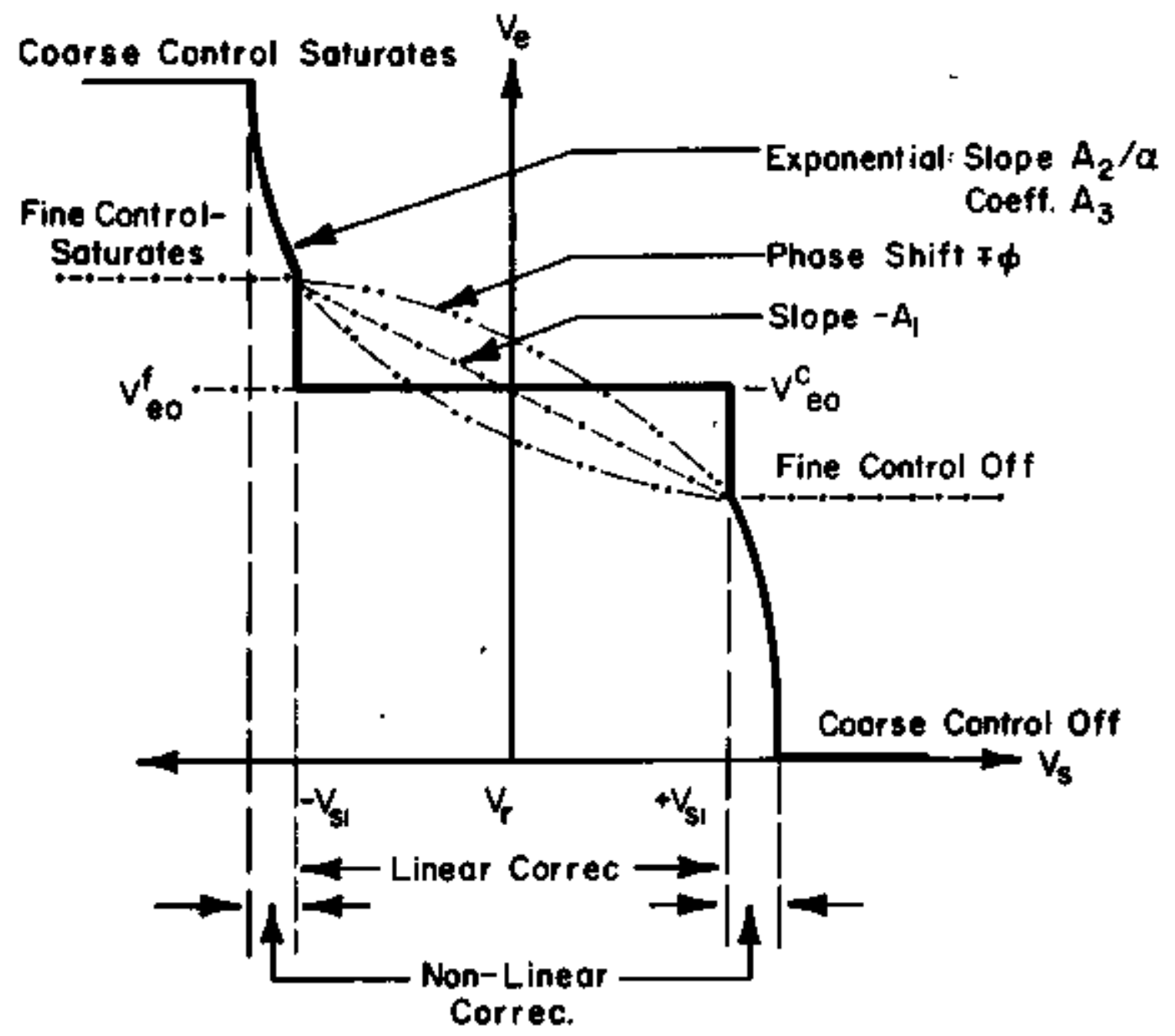

Non-Linear Driving Coorse Heater

-.-.-.-Linear Driving Fine Heoter

Figure G-1.

Linear and non-linear relationships between the error voltage $V_{S}$ and the correction voltage $V_{0}$. Hotice that hotter temperatures (less correction power) are to the right and colder temperetures (nore correction power) are to the left about the oulescent bias poillc. 
included meters to monitor the levels. The circuit is sketched briefly in Figure G-2 with all controls indicated. The functions that the andlog computer produces are:

$$
\begin{aligned}
& V_{e}^{f i n e}=A_{1}\left(V_{r}-V_{s}\right)+V_{e 0}^{f} \\
& I_{R}^{f i n e}=e^{+i \phi} \frac{\sqrt{V_{e}^{f i n e}}}{R_{f i n e}} \frac{a_{f}^{\prime}}{a_{f}}
\end{aligned}
$$

and thus:

$$
\begin{aligned}
& \tau_{\text {correction }}^{\text {fine }}=\alpha_{f}^{i} v_{e}^{f \neq n e} \alpha^{ \pm i \phi} \\
& v_{e}^{\text {coarse }}=v_{e 0}^{c} q_{3}\left(e^{\left.\alpha \mid A_{2}\left(V_{e x t}-V_{s}\right)\right\}}+1\right) \\
& I_{R}^{\text {coarse }}=\frac{\sqrt{V_{e}^{\text {coarse }}}}{R_{\text {coarse }}} \frac{\alpha_{c}}{\alpha_{c}}
\end{aligned}
$$$$
\text { is controled by }
$$$$
v_{s} \text { and the level } V_{s *}
$$

and thus:

$$
T_{\text {correction }}^{\text {coarse }}=a^{*} \mathrm{v}_{\mathrm{e}}^{\text {coarse }}
$$

The photos in Figure G-3 give an idea of the non-linearity possibtlittes of the coarse control. It was further necessary to filter the error vol tage into three components. The error voltage would have a slowly varying (O.C.) term that would correspond to a slow temperature drift of the sample through the critical region. This voltage should not be servoed, but instead, 

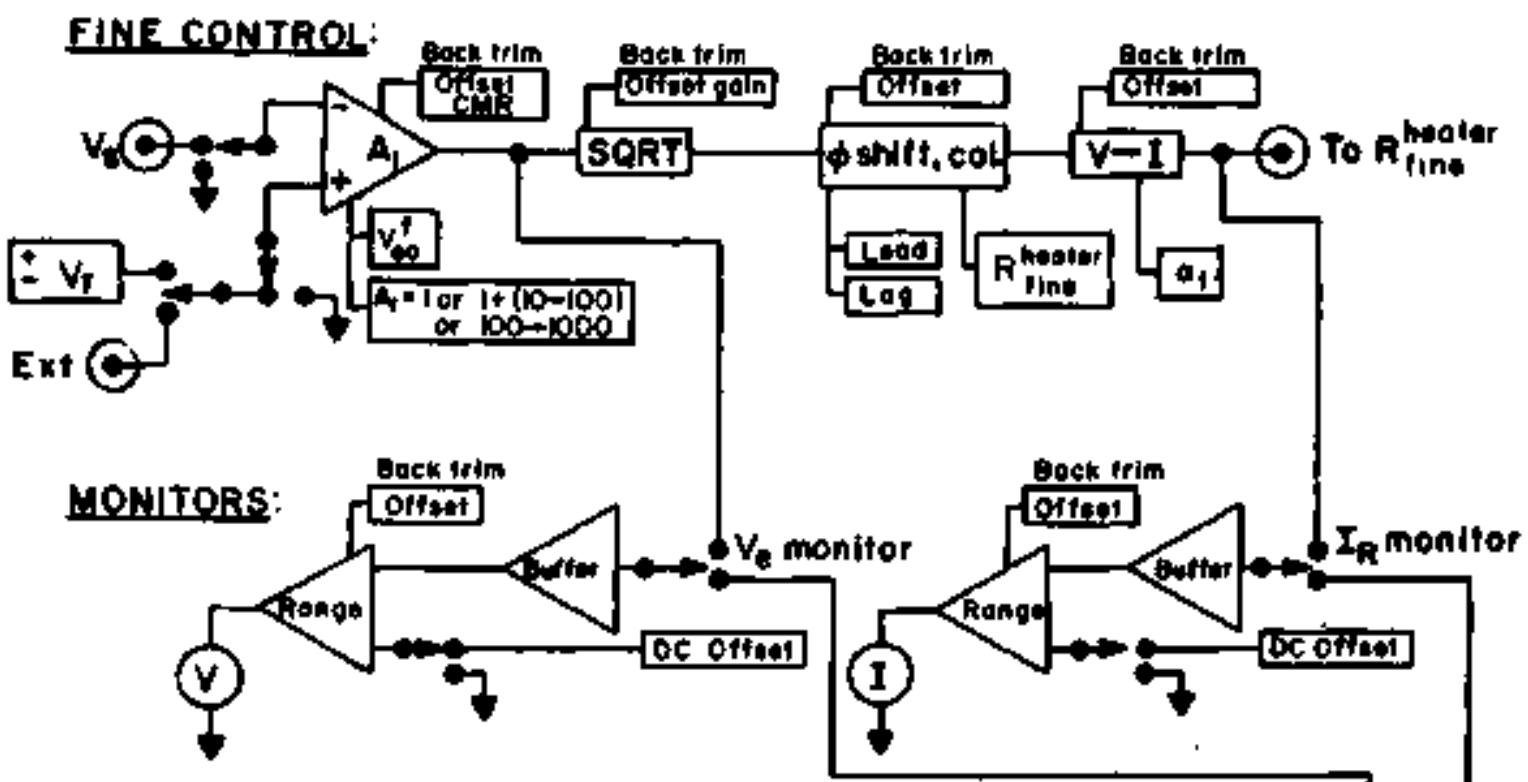

COAASE CONTROL:
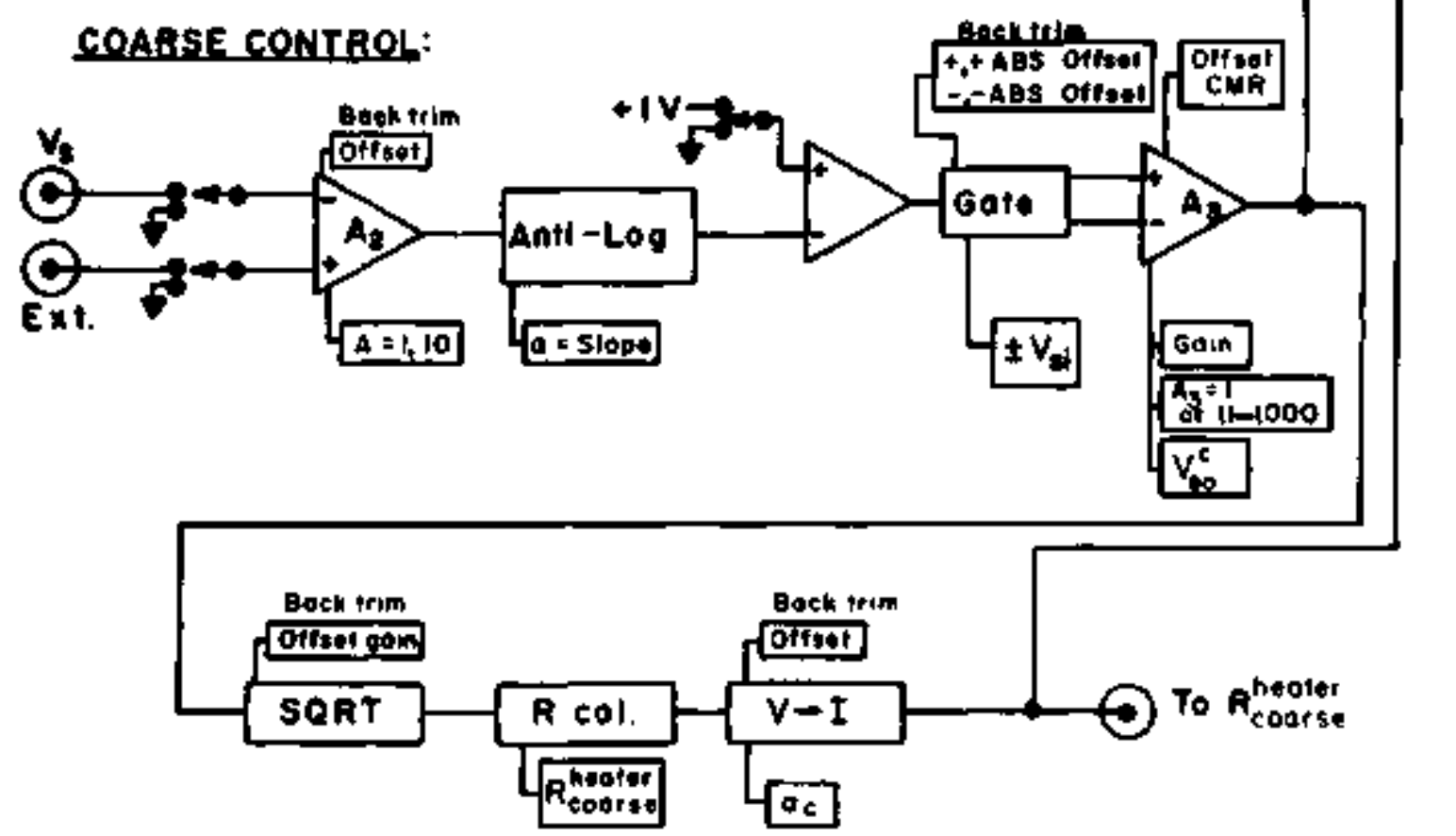

Figure G-2.

Block diagram of analog computer which was built to provide the correction curves of Figure G-1. Front panel controls are below the function and back panel controls are above the function. 


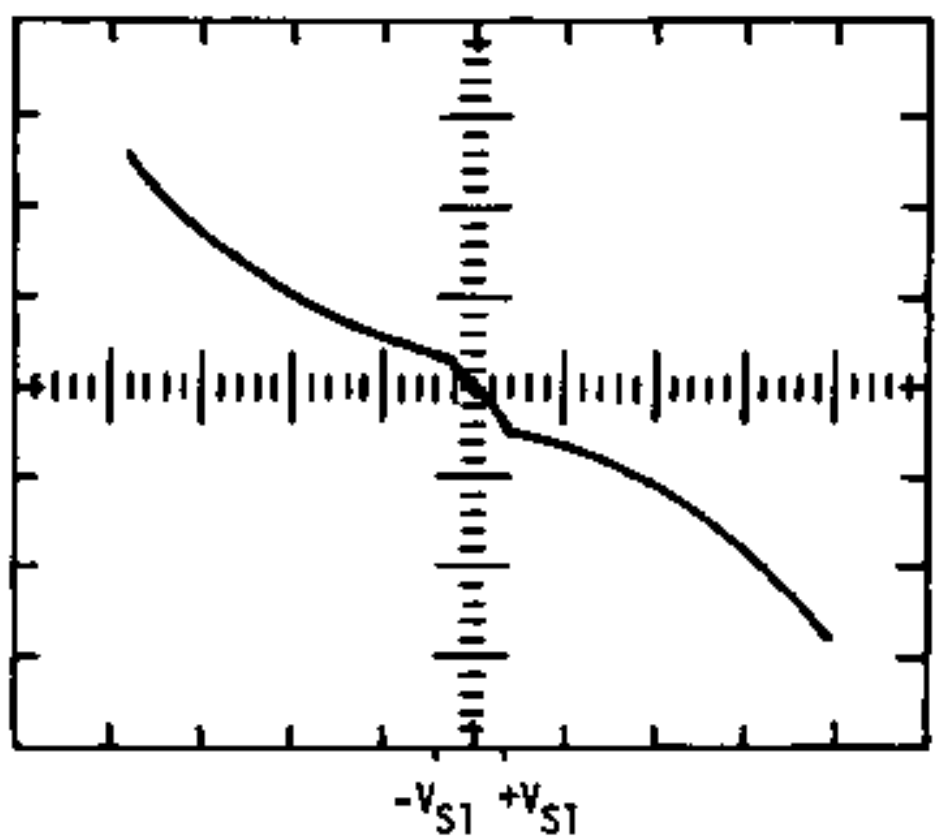

(a)

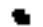

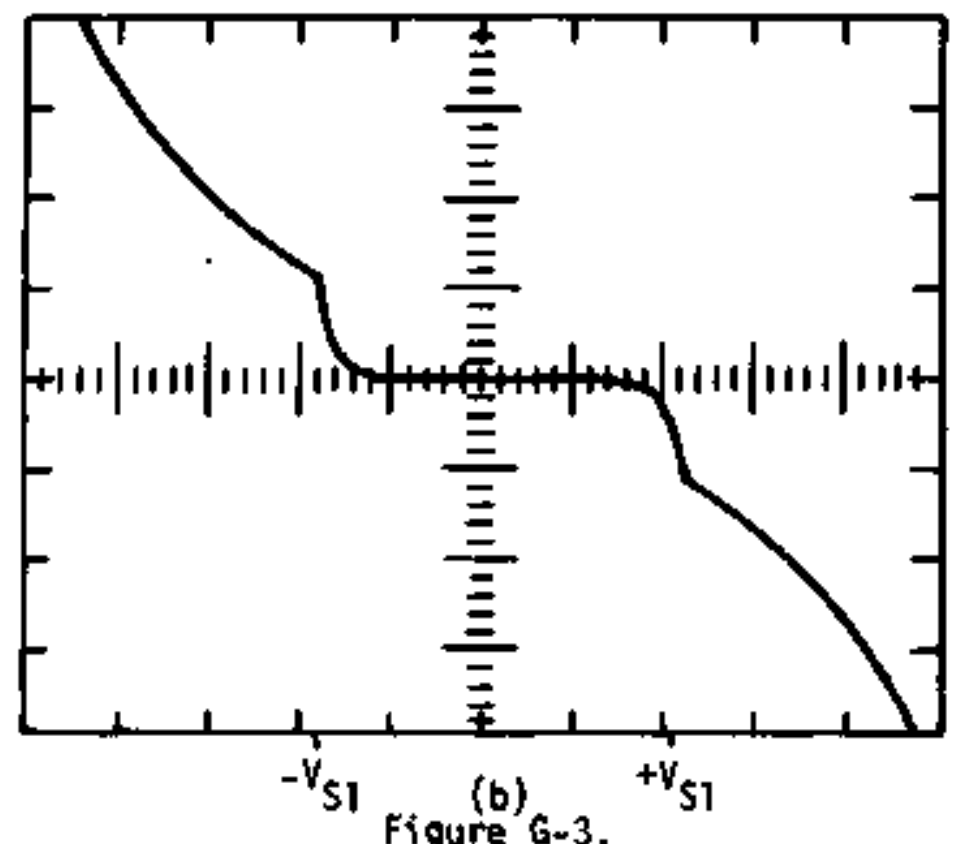

Osc $\$ 11$ is cope pfctures of the non-3inear functions produced by the analog computer of $F$ igure $G-2$. The horizontal axis is the error voltage $V_{S}$, and the vertical axis is the correction voltage $V_{e}$ (which is Tater converted to current). The figures are symmetric about the quiescent bias level. Figure a) has a low value for the gating voltage $\left(V_{S I}\right)$ and figure $b$ ) has a large value. 
It should be feedback to a self-balancing bridge (see A,C. Bridges section), and thus is used to cancel the offset voltage. A low frequency component would be used for the slow drifts and a high frequency component would be used for the fast themal feedback part. Originaliy the whole error signal would be fed Into the analog computer as a single error signal, but tater, the h1gh frequency part was fed into the 1 inear part, and the $10 \mathrm{w}$ frequency part was fed into the noin-linear part. The active filter box, shown in Figure 6.4 was constructed and accomplished this dissection of the frequency components of the error sfgnal. The complete block diagram of the semo system is shown in Figure G-5.

The perforwance of the overa]l servo system was poor. The electronics operated perfectly, as designed, but the main problens were in the complext ty of the syster and the rather slow thermal time constants associated with the teriperature sensor. The systen operated in a stable mode with time constants of the lock-1n at. values down to 10msec. One could change the gain, in both directions (higher and lower), to go into unstable operation. This indicated true closed loop feedback stability. But spurious noise would throw the system out of stability and required extensive effort to get back in a stable mode. Also, the temperature regulation was only on the order of $10^{-4} \mathrm{~K}$, which is quite poor by normal servo standards. To correct for the long thernal time constant between the sample block and the temperature sensor (Cryoca1), two sensors were mounted sympetrically on the block and well greased to $1 t$ 


\section{ACTIVE FILTER BOX}

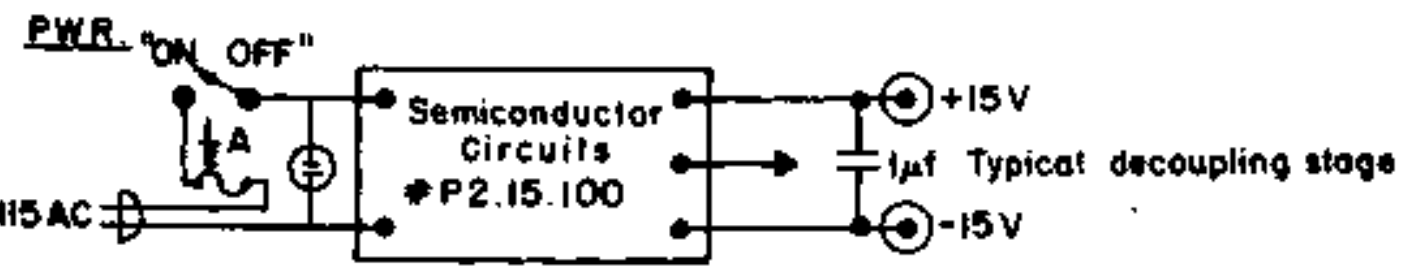

Of. FEEOBACK

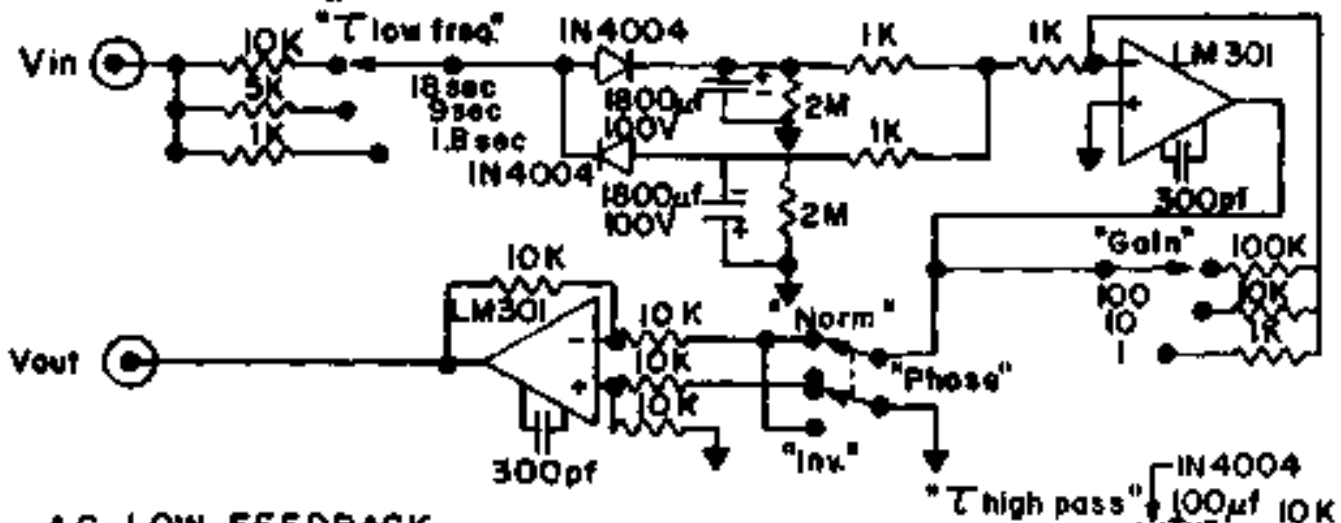

AC LQW FEEREACK

Vout

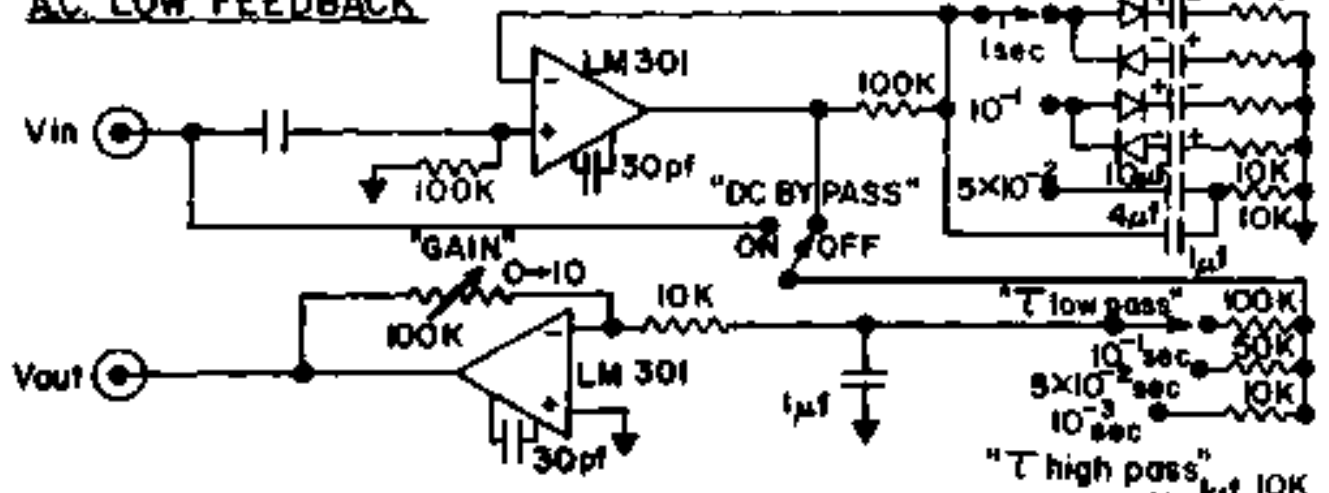

A.C. HIGH .FEEDQACK

"OC EYPASS"

vin
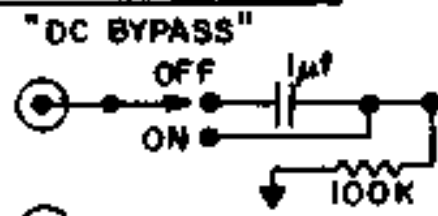

vout

Prin301 100K

"T high poss". 
Figure 6-5.

Complete block disgram of all components used in the temperature servo systent. The analog computer is sketched in Figure $\mathrm{G}-2$ and the active fiter is the circuit of Figure G-4. The self-balancing oridge is discussed in Appendix $A$ and the low temperature system is discussed in Appendix C.

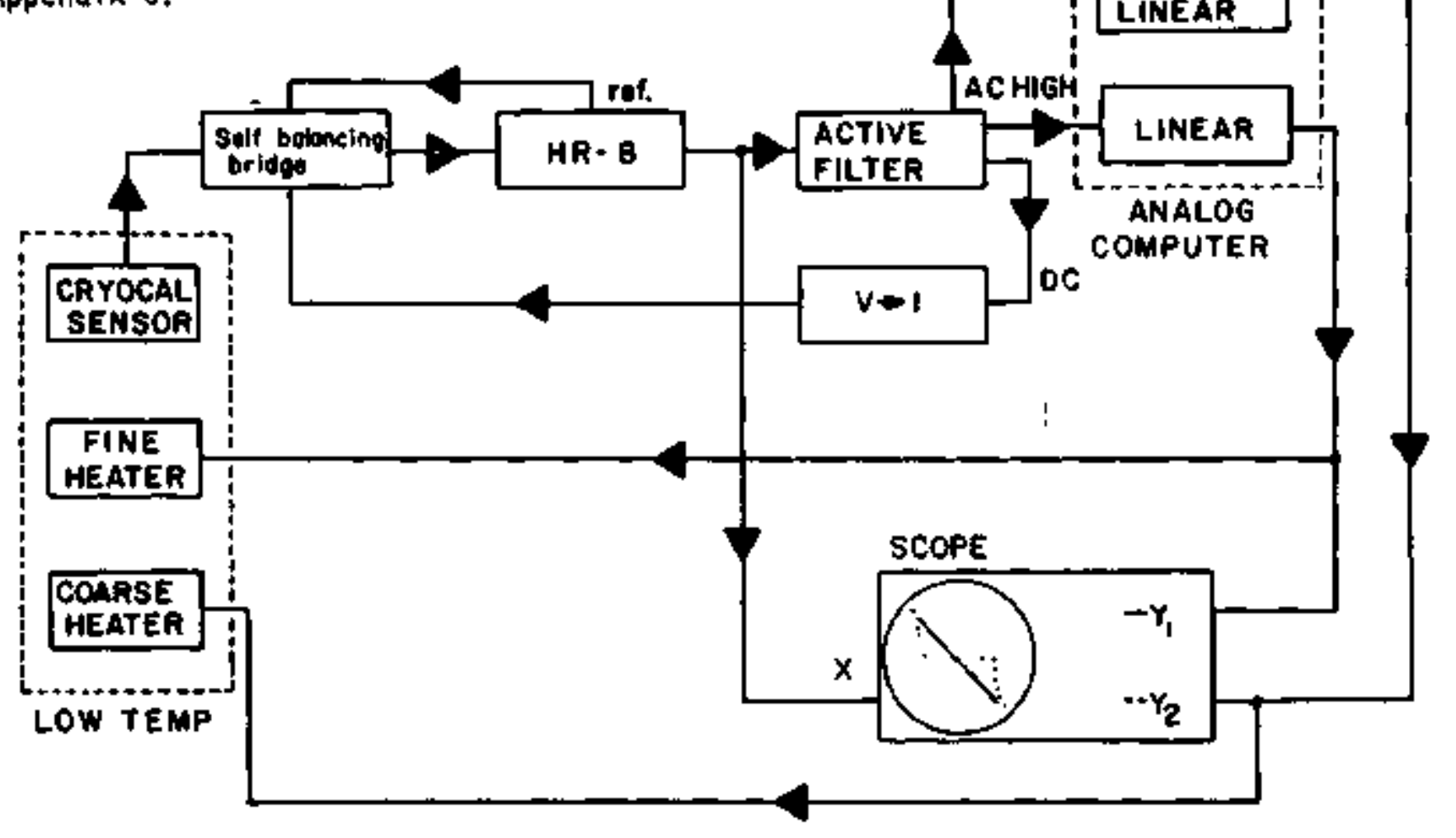


(one was a Cryocal Standard; the other was a Cryo Resistor Standard\}. Both sets of Teads were Isolated with superconducting spliced wires and clamped with copper to the sample block in a jens paper sandwich. In this way, not only would the thermal time constant of the sensor be reduced, but also one could independently monitor the temperature with a sensor outside the feedback loop. The results indicated that in general a servo feedback system behaves as a self-balancing bridge, in that the heater will ratse or lawer the power to correct the temperature seen by the sensor. This may nat be the true temperature errors exhibited by the sanple block, since it is not in perfect theranal contact with the sensor. As it turned out, the sensor in the: feedback 1000 would indicate thermal fluctuations of oniy $5 \mu K$, but the actual fluctuations as measured with the other sensor were on the order of $30 \cdot \mathcal{K}$.

In genera 1 , the servoing would improve the long term drifts of the sample block extensively, but at the cost of introducing short term thermal fluctuations in excess of background fluctuations. Because of the increased thermal noise introduced by the servoting. and the comiplexity of operation, the servoing was used only when taking polnt by point data, and in general the inner can was servoed by conventional bridge from an independent sensor, and the sample block was allowed to sit at a temperature determined by $D$. C. power leve1, and not servoed at all. This gave an accuracy of about $10 \mu \mathrm{K}$ fluctuations to the overall experiment. 
Appendix H. Sample Preparation and Resistive Transitions

The superconducting f11ms studfed were "dirty", granular, aluminum, thin fi\}ms ( $\sim 1000 \AA$ thick) prepared by vacuum evaporation onto a number of different room temperature substrates. Since there has been extensive experimentat, as well as theoretical work into the resistive transition of "dirty", aluminum thin films, it is 1mportant to show where the sariples studied agree wt th other workers. But, due to the diversity of other workers' sample preparation methods, and the undefined parameters in their work, the correlation will be somewhat limited. The undefined parameters include effects of thickness, grain size, strains, and "dirtiness" in raising the transition temperature $\left(T_{c}\right)$. They also include effects of current densities (A,C, and D. C.), and low magnetic fields used in the measurements that change the width of the transitions. And finally. effects of the chemical enviranment during sample preparation become undefined parameters in the samples prepared and studied by other workers. No one group working with dirty aluminum flins has charactertzed all these parameters for the films they studied. Thus, this section will point out the effects of the parameters mentioned above in the films studied, with a partial attempt at correlating the gross features of the resistive transitions.

If one evaporates aluminum in some impure atmosphere at a slow rate ( $30 \mathrm{~N} / \mathrm{sec})$, one will get a granular aluminum film. The 
grains vary in size, byt the fi\}ms studied had grains on the order of $400 \AA$ (see Fig. $\mathrm{H}-1$ ), and have $T_{c}$ s which are consistent with the $T_{c}$ 's of other work. ${ }^{47}$ The method of providing an impure atmosphere varles widely and may not be obvious, even to the person preparing the sample. Some workers co-evaporate S10 to difty the films. Others have a residual $\mathrm{O}_{2}$ background. The effect of the $\mathrm{O}_{2}$ is to first provfde seed centers for granular formation (even $\mathrm{He}^{4}$ works ${ }^{37}$ ) and second to oxtdize these grains after formation (accomplished later upon exposure to air, if not initially). These processes are governed by other processes too (the "undefined parameters" referred to earlier). The $\mathrm{O}_{2}$ method was chosen for the author's films due to the Inconvenience of working with SiO. One undefined parameter in the $0_{2}$ process is the residual water vapor in the evaporator system during evaporation. In this laboratory. Rfchard Carlson was making SKS tunneling Junctions where the insulating layer was a $5 \AA$ layer of $\mathrm{Al}_{2} \mathrm{O}_{3}$, and he found that this thickness was drastically affected by the relative humidity of the room. Analysis with a partial pressure gas analyzer showed that oxide layers were dependent on the amount of water vapor present in the vacuum system. Only when going to 4 day system bake-outs at about $120 \mathrm{C}$ could this background be removed. But even evaporation from $\mathrm{Al}_{2} \mathrm{O}_{3}$ coated boats introduced $\mathrm{O}_{2}$ and $\mathrm{H}_{2} \mathrm{O}$ vapor during evaporation. These water vapor effects are probably due to the $\mathrm{OH}^{-}$radicals' tunneling ability to create channels in an $\mathrm{Al}_{2} \mathrm{O}_{3}$ layer that can expose the aluminum to further oxidation. Thus any films made by evaporating aluminum in an oxygen background will be greatly affected by the background water 


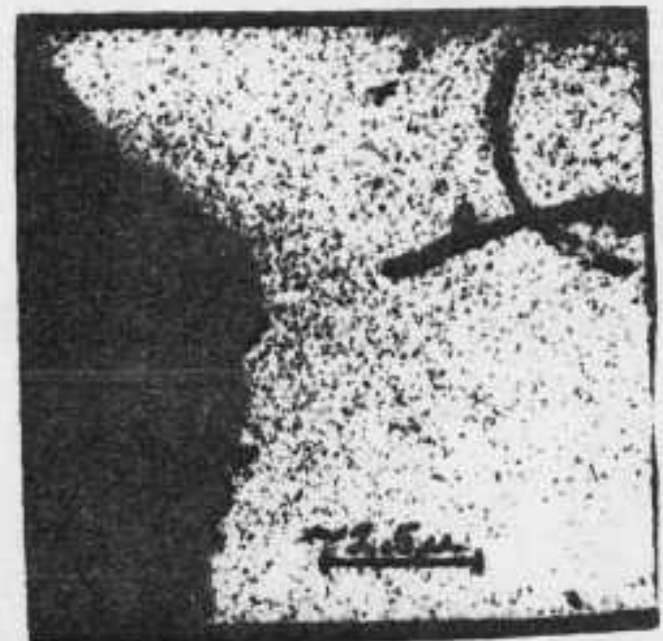

a.) Ran \#33

Approx, 6800 Magniticatiti

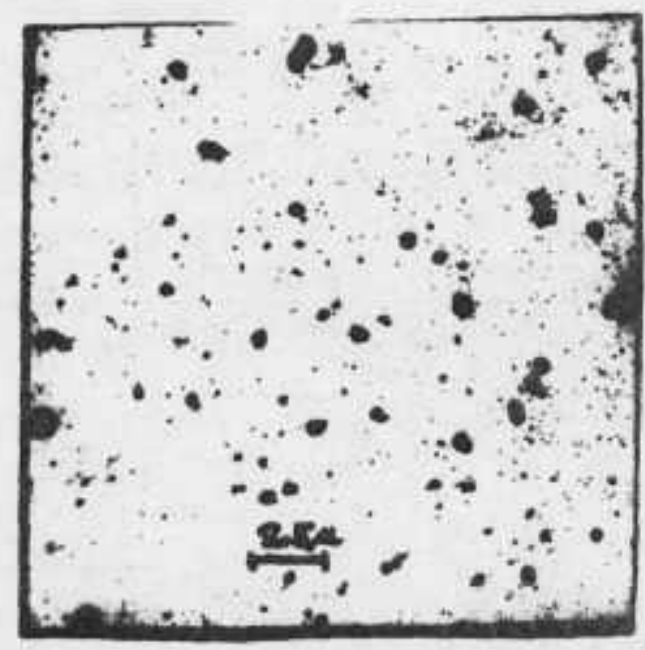

b.) $R$ in $\# 32$

Apptox. 4000 Magnification

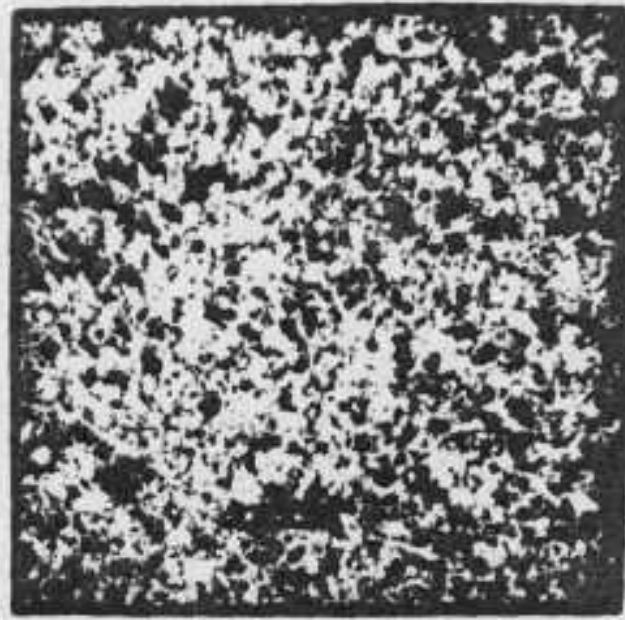

c.) Rurn $\$ 24$

Approx. 4000 Magntication

Figure H-1. TEM microgiaphs of $1000 \AA$ aliminum $: 11$ ms on mica (a). glass (b.), and self-supporting substiates (c.). The crevice in $(\mathrm{a}$,$) is a seratch. The large daik spots in$ (b.) are foreign particles. And the coarse gi id (that looks like little squares) of (c.) is a replica of the salt substrate that textured the ilm and remained after it was remounted. Notice that the grain sizes are about the same for each substrate. The grain sizes varied between $200 R$ and the $111 \mathrm{~m}$ 's thickness (1000 $\mathbb{R}$ ). These micrographs were made with the assistance of Keith Munson and Tom Hutchinson. 
vapor in the evaporator, which will vary considerably from laboratory to 1aboratory.

The sataples were prepared predeminately by evaporating aluminum from an alunina coated molybdenum boat that was resistively heated. Evaporations were at rates of from 10 to $50 \mathrm{~h} / \mathrm{sec}$ through a mask over the substrate, with a substrate to source distance of about $40 \mathrm{~cm}$. It should be noted that the rate intght also affect the time the grafns spend in the oxidizfng atmosphere. This atmosphere varied from nothing coutside of the residual $\mathrm{O}_{2}$ at $10_{\text {torr }}^{-7}$ to $10_{\text {torr }}^{-4} 0_{2}$, but was typically $4 \times 10_{\text {torr }}^{-6}$ ). Some periods of sample preparation were marked by a very low $T_{c}$, which led to the addition of drops of water before the system was baked out (at $120 \mathrm{C}$ for $12 \mathrm{hr}$.), in order to lesve a partial pressure of water vapor during evaporation, and thus enhance the $T_{c}$ of the films. These methods of sample preparation gave $f 1 \mathrm{Im}$ resistivities ranging from $6 \times 10^{-5} \Omega \mathrm{cm}$ to $4 \times 10^{-7} \Omega \mathrm{cm}$ and $\tau_{c}$ 's ranging from $1.54 \mathrm{~K}$ to $1.90 \mathrm{~K}$. More typical samples were of resistivities of $1.5 \times 10^{-5} \Omega \mathrm{cm}$ and transition temperatures on the order of $1.60 \mathrm{~K}$, for thickness of $1000 \stackrel{0}{\mathrm{~A}}$. Since the mean free path in the films is related to the resistivity ( $\left.e_{\text {eff }} \sim \frac{4 \times 10^{-12} \Omega \mathrm{cm}^{2}}{\mathrm{P}_{n}}\right)^{38}$, these flims had mean free paths of about $20 \%$.

These heavily oxidfzed films might have been actually quite dirty, and thus had low $T_{e}$ 's because they were on the far side of a hump in the empirfcally observed relationship between $T_{c}$ of the film and the level of oxidation. ${ }^{42}$ In fact, one extremely dirty film (30 drops of water in the evaporator) exhibited a negative slope in 
its resistivity vs. temperature, and it never did go superconducting (down to $1,4 K$ ), thus indfcating a semi-conductor behavior. It was also found that the heavity oxidized films exhibited considerable effects in the resistive transftion from an addftional D. C. current $\left(\sim \mathrm{TOA} / \mathrm{cm}^{2}\right.$ ) used in the medsurement. For some $f 11 \mathrm{~ms}$ this effect was quite sensitive, where only an increase of $10 \%$ tn the current would bring about the effect. The effect was to add slight bumps and glitches ("kinks") in the resistance vs, temperature curves, which were nomatly reasonably smooth. If the A.C. current was made equal to the D.C. current, the effect was almost el Iminated. This effect might be related to some sort of Josephson tunneling between gratns ${ }^{45}$ becoming partially coherent at certain values of $0 . C$. current.

The films were evaporated on predeminately mica substrates. These substrates were cleaved from optical quality mica to a thickness of typically $\&$ mil. The mica was glued around $i$ ts edges to an alumina substrate that had $\mathrm{Pb}$ posts attached, and the alumina substrate was in turn glued to the sample block (see Appendix $\mathrm{C}$ and Fig. H-2). After the aluminum film was evaporated onto the mica, silver leads ( $\sim 50 \AA$ thick) were evaporated from the $f t l m$ to the posts, and then these leads were overlayed with a $10,000 \&$ evaporated $\mathrm{Pb}$ coating. Finally an Indium solder bead (Indium Alloy \#2 solder and Flux 2 , available from Indium Corp. of America, Utica, New York) was drawn between the Teads and the $\mathrm{Pb}$ posts to complete the four terminal circuit to the film. 
The filas were made in both circular and rectangular geometrtes (see Fig. H-2). The circular films had a 2 terminal measurement, and wile the overlapping leads essentially removed edge effects (and hence no scribing was needed), they provided a nonunfform current density in the film. It is interesting to note that the ratio of width/length (used in calculating $R_{\square}^{N}$ ) can be calculated almost exactly for the circular geonetry, and it is independent of the diameter of the circle and is equal to 1.568 . Because of extra parameters brought in by nonuniform current densities, the film geometry was changed to that of a rectangular form (see Fig. H-2). The edges were scribed because of possible adge offects in the transition ${ }^{46}$, and a 4 terminal measurement was made. Later, a snake scribe was added to give a factor of ten greater resistance, which tmproved signal coupling and sensitivity in eleotrical measurements.

The films on mich substrates had predominantiy multiple transitions (more than one $T_{c}$ ). It was at first thought that this was due to the layered nature of the mica acting like on edge effect and thus creating a $T_{c}$ for steps in the mica; and thus the overall transition would have multiple $T_{c}$ 's. But, films on thin glass ( $\sim 3$ mil, thick) also had multiple transitions. Even special consideration was given to examining the mica used. The nica was examined between crossed polarizers, at a large angle, to determine the existence of any differences in the thickness of the substrate, and only uniform substrates were thus used. This precaution did not 
SAMPLE GEOMETRIES

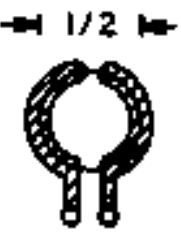

Circulor $\mathrm{flim}$ 2 termingls msctibe

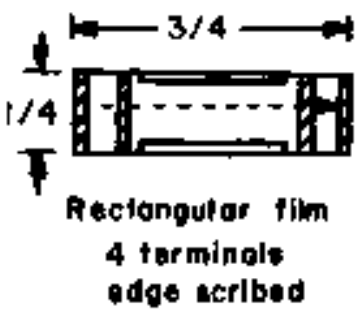

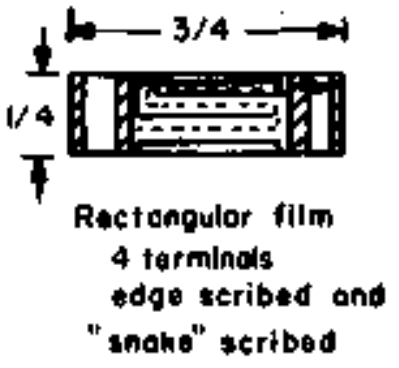

\section{SELF-SUPPORTED METHOD}
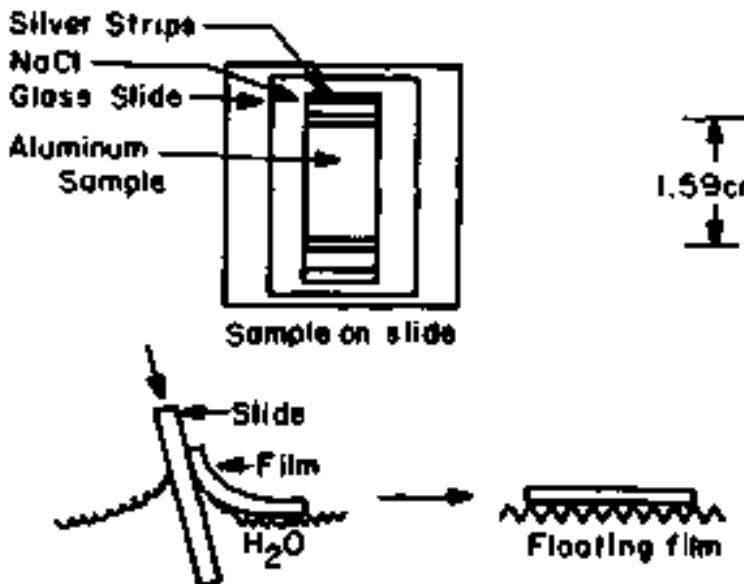

Flooting flim
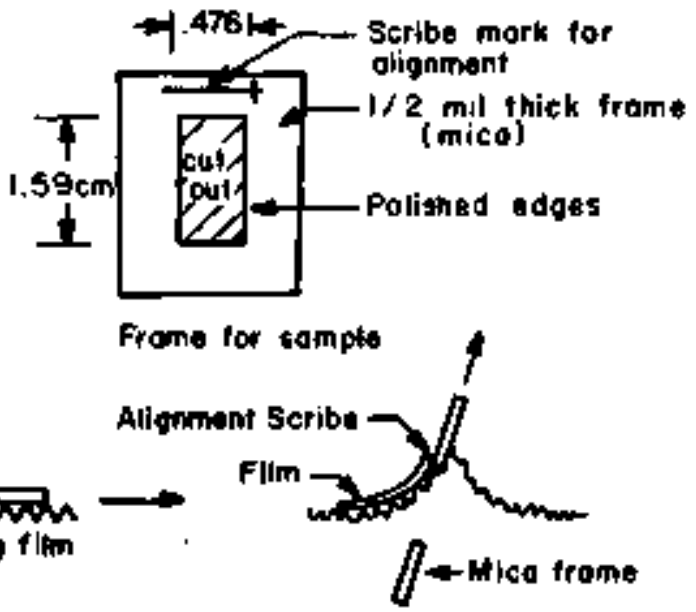

Top vlew

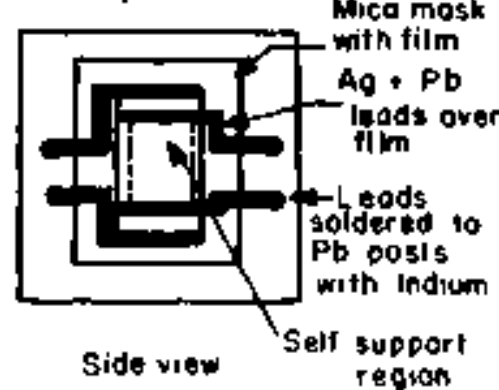

NN $/ O U T$

Evaporoter

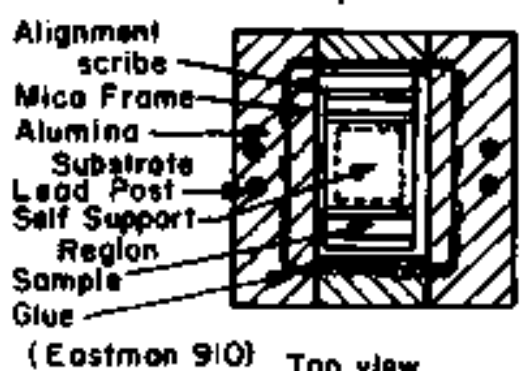

(Eostmon 910) Top viaw

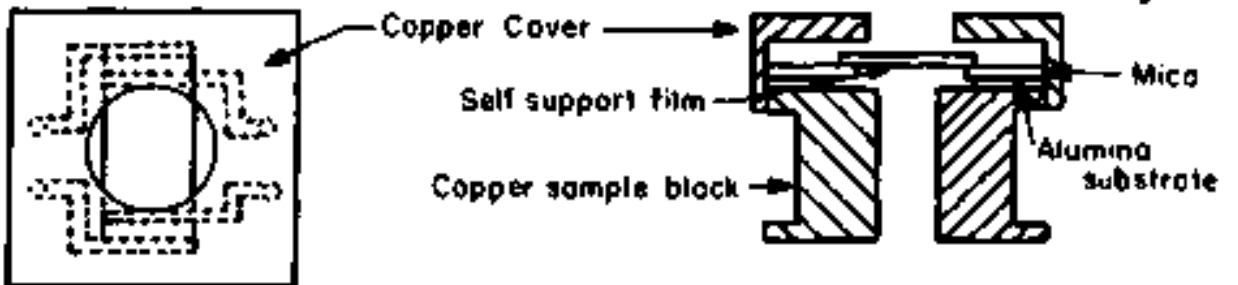

Hounted sample on tomple block with thermol shield

Figure H-2.

Sample geometries and self-supported fitm preparation method. 
inprove the transitions. When the measurements of the D.C. thernal conductivity of the mica were made, where the whole substrate was gived un1formly to a flat copper block, the problem of multiple transitions was solved. The previous substrates were held only on the edgos and could flex during the cool down from $300 \mathrm{~K}$ to $4 \mathrm{~K}$. These stresses created multiple transitions. Two films in a row, on rigidly held substrates, had single transitions. The sensitivity of the transition to strains in the substrate has been suggested by Testard ${ }^{44}$ and presents the possibility of incorporating this "problem" into a very sensitive pressure gauge (although highly non-1 Inear).

Films were aiso made that were self-supporting. Previous workers (ref. 39) have made self-supporting f11ms with a variety of metals by variety of methods, at though none of their films approach the rather large size in area of these films $\left(\sim 1 \mathrm{cos}^{2}\right)$. Films were made initially on dissoivable Naci substrates. They exhibited large contact resistances due to extensive oxidation of the aluminum surface, probably from the $\mathrm{Cl}^{-}$of the salt tunneling into the surface. A number of attempts were made to remove the contact resistance problem from this surface oxidation. A single NaCl crystal was used as a "plug" in a hole drilled in an alumina substrate, with the crystal cleavage plane parallel to the substrate surface. In this manner, the film and leads could be evaporated at one time and the substrate (NaCl crystal) dis5olved away later by a method similar to that of ref. 40 , leaving the center portion of the film over the hole in 
the substrate, free standing. The problem wfth this method was that the boundary between the crystal and the substrate had slight crevices that the aluminum film would not quite cover, and thus the film was barely attached to the edges of the alumina substrate. A thin collodion substrate was also tried, but due to problems in surface tension and dissolving the substrate away after evaporation, this method was also discarded. The method that was fina?ly used is simflar to that in ref. 41. About $4000 \AA$ of $\mathrm{NaCl}$ was evaporated onto clean glass slide. The film was evaporated onto this substrate. Both circular (up to $\mathrm{z}^{\prime \prime}$ in diameter) and rectangular ( $h^{\prime \prime}$ by $3 / 4^{\prime \prime}$ ) films were evaporated onto this substrate. Silver "stips" were evaporated onto the rectangular films to reduce contact resistance from leads attached later (although these strips were not always necessary). The slide was very carefully towered into a still bath of distlifed, afr free water (see Fig. $\mathrm{H}-2$ ) with about 5\% special denatured alcohol added. Isopropyl alcohol was also used, since it factlitated the film removal by causing less of a water drop to adhere to the film and tear the film, but it also reduced the surface tension of the water and caused the films to sink. The fijms would 81so sink on very humid (rel. hum. $60-70 \%$ ) days, due to less of a vapor-liquid boundary on the surface of the water. An opposite problem was the electrostatic charge that would cause the film to Jump back upon itself during periods of very low humidity in the winter. After the film has been floated fres of the sifide, it is comparatively strong in the water. But, it is crucial to lower 
the film into the water at about $20^{\circ}$ from the norkal angle and at a steady, continuous speed. Any jitter w1ll cause milnute tears of the film or fts odges, which will later, upon drying, tear the entire film (it is advised to meditate awhile before trying to float off the fijms). The film then was picked out of the water by alfgning the edge up on a mark on a mica frame and stowly raising it vertically put of the water. The films were at first coated with Napthalene vapor to strengthen them for removal, but atthough the Napthalene sublimates at room temperature, it hindered the films from sticking to the mica, and it also added to the surface contact resistance. Bacause the mica is very thin, and because it was raised slowly and vertically out of the water, very littie water adhered to the film to "hang" onto it and cause it to rupture. The mounted $f t 1 m$ was aried by holding vertically in front of a high intensity lamp. The mask was positioned on the alumina substrate and glued around the edges with Eastman 910 glue (because the glue vapor will corrode the film, use a orinimal amount). After the glue had dried, the sample block was put back into the evaporator, and leads ware evaporated over the "strips". The block was renoved from the avaporator (gas being added and renoved slowly from the system so as not to rupture the fi $7 \mathrm{~m}$ with a pressure gradient), and the leads ware soldered to the $\mathrm{Pb}$ pads. The blockwas covered with a thermal shleid and installed in the cryostat. A detector was positioned under the film to detect light passing through a ruptured film after cool down. The rectangular films tended to pull away from the long ends of the frane after cool down (due to larger 
thermal contraction), white circular films had less offficulty. Films below $800 \AA$ thick were impractical to work with. The films of $1000 \AA$ thick were still thin enough to transmit vistble light, since they were on the order of the metallic skin depth in thitekness. The whole procedure involves a decreasing percentage of success. One has ane in four chance of floating off a good film. One has a one in three chance of remounting the film and gluing it onto the alumina. After the leads are evaporated on, one has still a $50 \%$ chance of tearing the film by the I.R. heating during soldering. Then, upon cool down, the film still can tear, due to the thermal gradients. For this reason, an optical detector was positioned below the f1lm to detect torn film. Finally, upon completion of the experiment, one has a slight chance of the film surviving the warm up to room temperature. One film survived the complete process outlined above wf thout appreciable tearing, whtch also had data taken on it for heat capacity.

The measurements made on seif-supported films showed that almost an equal thermal isolation was obtained in the experfment using high heating frequency with the mfea substrates, and due to the difficult nature of the self supported films, they were no Tonger studted. The films on mica were made on substrates of thicknesses from $2 \mathrm{mil}$ to $\sim .1 \mathrm{mll}$ and had results simtlar to the results of the self supported flims, which seemed to support the ided that not only is the transverse thermal conductivity of mice very low, but also that the high frequency of heating 
essentialiy removes the mica from the measurement.

One self-supported film had an unusual feature in its rosistive transition. This feature is umexplafnable, and because it is so unusual, one hesitates to mention it. But, because measurements of the transition were made over seventy times, on two separate runs, and because the effect is a large percentage of the signais measured, one is forced to berieve it as being physical. Fig. H-3 shows the resfstive transition of sample \$24 (see fig. H-2 for a micrograph of the same film). This film had an unusuat luster to it, f.e., very shiny and spectacular, as compared to the usually grayish self-supported fllms. The transition has in it "notch" that appears as if it is subtracted off from the usual resistive transition. All the discrete details in the notch are reproducible. During both runs, a gas leak caused a very slight He ${ }^{4}$ beckground $(P<1 m$ ma) that was steadily decreasing during the run (because of its being continually pumped on). The notch appeared after a few hours of running. and it moved up and down the transition with time. Figure H-4 shows successive transitions with the times between measurements 1ndicated. This movement was periodic, with a period of about three hours and with the maximum temperature reached with each period decreasing w1 th time. The addition of $\mathrm{He}^{4}$ gas at a later point removed the effect. The $f 41 \mathrm{~m}$ was destroyed during thermal cycling in a low temperature attempt to fix the gas leak. One might make * thermal runaway argument involving the $\mathrm{He}^{4}$ gas to explain the results. Or possibly a metastable state existed in the transition, as predicted in $1-D$ by Tucker and Halperin. ${ }^{48}$ 


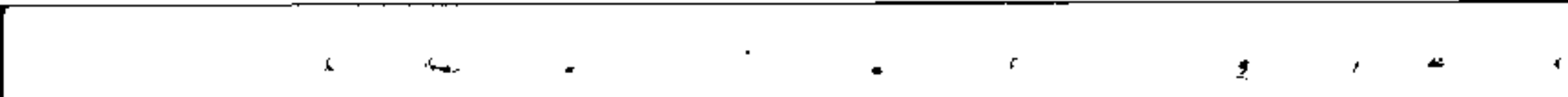

Figure H-3, Resistive Transition of a Self-Supported Film.

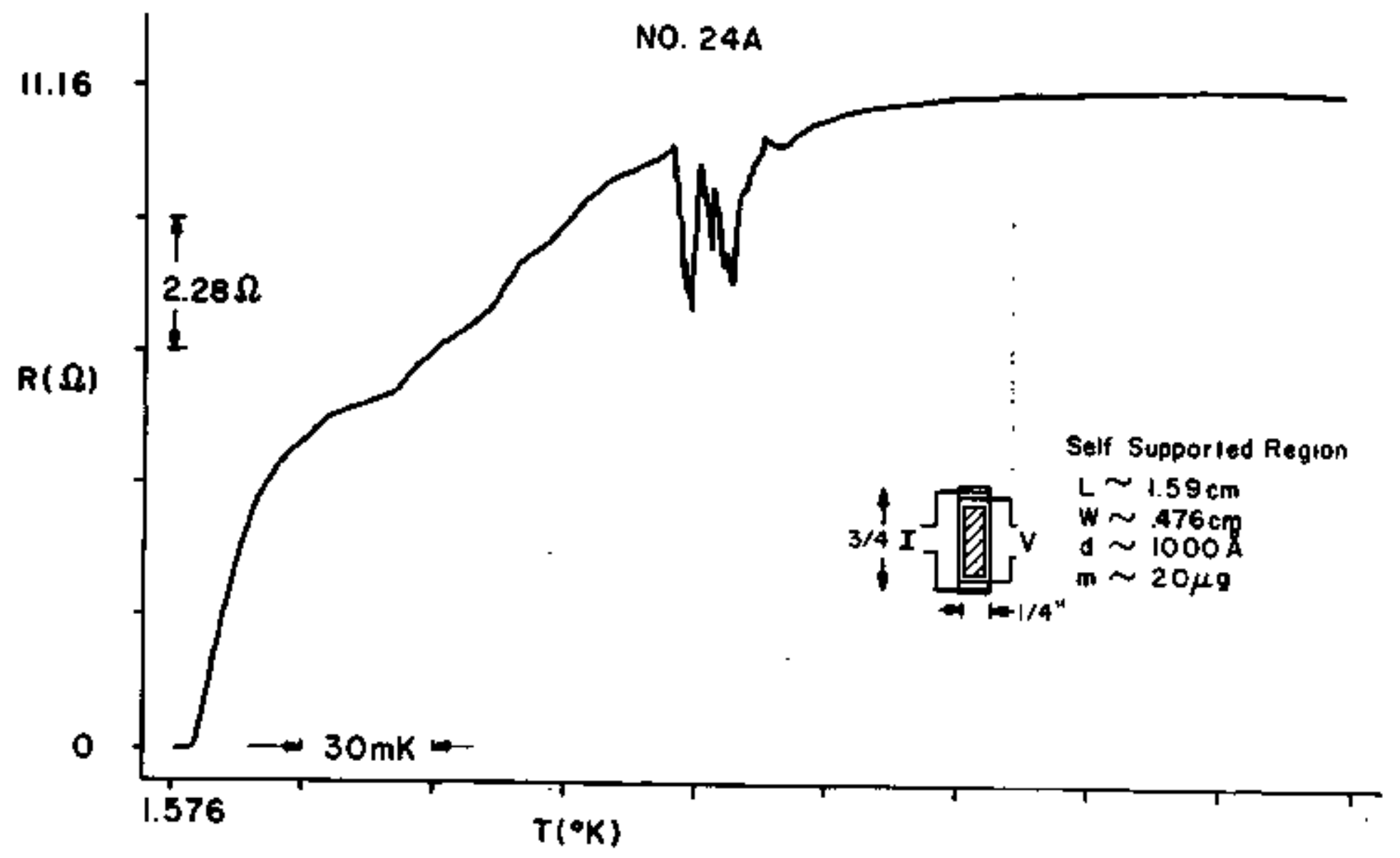




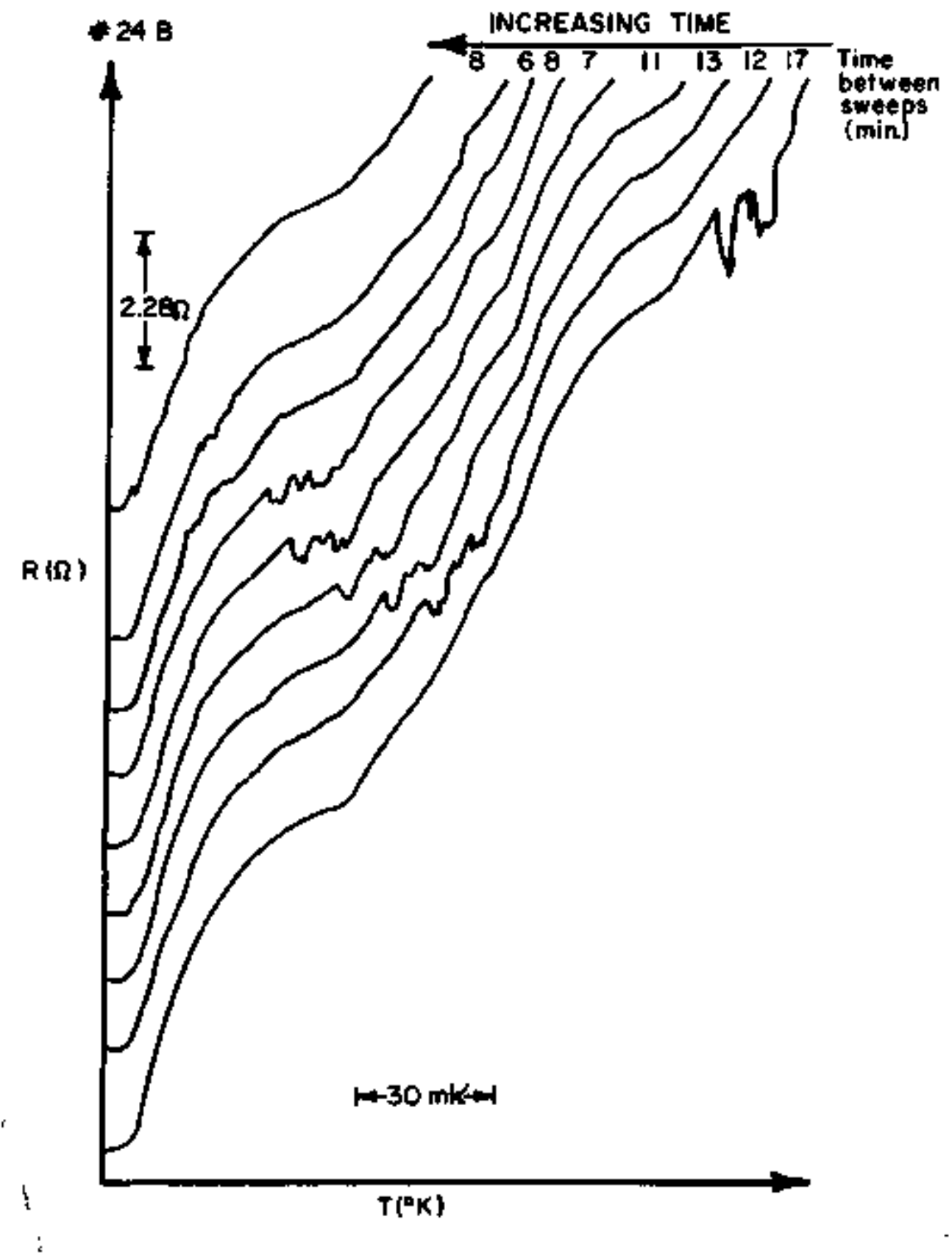

Figure $\mathrm{H}-4$.

Successive resistive transitions of the fllm shown in Figure $\mathrm{H}-3$. Notice the changing location of the "notch" in temperature for successive plots in time. 
The effect was not seen in a normal "greyish" self-supported film's transition where no He ${ }^{4}$ gas was present (run $\$ 23$ ). This strongly suggests some form of thermal runaway.

Magnetic field effects on the filas " transition were also observad. One of the dirtier filas (sample $\$ 30$ ) exhibited a slight increase in the width of the transition for small 0.C.currents $\left(10^{-6} \mathrm{amp}-10^{-5} \mathrm{amp}\right)$. The widening of the transition was 1 inear and had a slope of about $\frac{d I}{d(\Delta T)} 1.6 \times 10^{-3} \mathrm{amp} / \mathrm{K}$. The fietds associated with these currents are possibiy only on the order of tens of nanogauss, so that the increase in the width of the transition may be due to some other effect. Out this points out a problem in increasing current densitfes to measure Towering resistances. Larger magnetic fields were desired ( $\sim 10$ gauss) in the experiment, and a relatively simple solenoid was used in run $\$ 39$ (dianeter $\sim 3$ ha", length $\sim 12 ", \sim 108$ turns $)$ to apply a perpendicular field (al though quite nonumiform, about $10 \%$ over sample region) to the sample. Figure H-5 shows three resistfve transitions for three values of magnetic field ( $\left.H_{2}\right)$ in a relatively clean (less oxidized, not to be confused with "pure") film. The transition is broadened with a long tall on the low $T$ side, and it is moved to a lwer $T_{c}$ by the increasing magnetic field values. One can calculate the coherence length from these curves in the 1 imit of $\left.\left(T-T_{c} / T_{c}\right) \ll\right]$. Then from ref. 49:

$$
H_{C 2}=\frac{\Phi_{0}}{2 \pi} \quad \xi^{-2}(T) \text { and } H_{2}=H_{C 2}
$$


Then

$$
\begin{aligned}
& \frac{d H_{\perp}}{\partial T}=\frac{\Phi_{0}}{2 \pi} \quad \xi^{-2}(0) \frac{d}{d T}\left(\frac{T-T_{c}}{T_{c}}\right) \text { for } \frac{T-T_{c}}{T_{c}} \ll 1 \\
& \Delta H_{\perp}=\frac{\phi_{0}}{2 \pi} \frac{\Delta T}{T_{C}} \quad \xi^{-2}(0)
\end{aligned}
$$

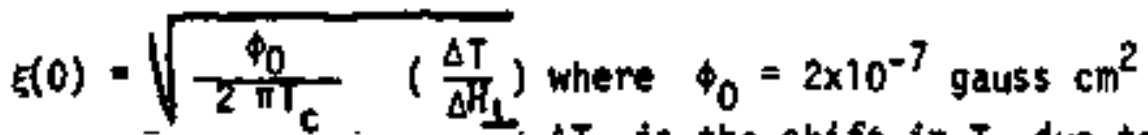

$$
\begin{aligned}
& \Delta T \text { is the shift in } T_{C} \text { due to } \\
& \text { the field } \Delta H \text {. }
\end{aligned}
$$

For the film of Fig. H-5, this becomes:

$$
\xi(0)=\sqrt{\frac{\left(2 \times 10^{-7}\right)}{(2 \pi)(1.81)\left(9 \times 10^{2}\right)}} \text { an }=440 \AA
$$

And since for dirty superconductors:

$$
\xi(0)=.85 \sqrt{\ell_{\text {eff }} \xi_{0}} \text { where } \xi_{0}=1.6 \times 10^{4} \AA \text { for al uminum }
$$

This gives an effective mean free path of $16 \hat{A}$. Using the expression used earifer to predict the $\ell_{\text {eff }}$ from the normal sheet. resistance ( $R_{\square}^{N} \sim i .5 \mathrm{R} / \mathrm{sq}$ ), one finds an estimated $e_{\text {eff }} \sim 26 \AA$, which is not in too good agreement,probably because the resistivity method of ealculation is not as accurate. (The work of ref. 50 measure an leff as low as $1.6^{\circ}$ in simflar films). Thus the films resistive behavior in small magnetic fields is in reasonable agreasent with theory and other worker's results (ref. 50).

The resistive transitions were measured in a dynamical way. 

The film was biased with o low frequency $(41.5 \mathrm{~Hz}$, and thus Tittle of an out of phase cormponent) constant current source, and the voltage produced was phase sensitive detected. The resulting output voltage was scaled to the bridge-measured, normal resistance of the film. The off null voltage from a bridge measurement of a cal fbrated germanium resistor (see A.C. Bridges Appendix) was scaled to represent temperature. Thus, by making an $x-y$ plot of these two voltages, while the temperrature of the sample was steadtly increased, one could obtain a 1st order approximation of the resistive transition. As a check on this method, a point by point plot was done using two nulled A.C. bridges, one on the film and one on the thermometer, to measure the transition. Also point by point plot was done using O.C. voltage measurement (averaged for both positive and negative currents, to remove emf effects). Al1 three methods agreed quite well. The main error in the vol tagevoltage plot is associated with the sightly non-linear relationship between temperature and the off null voltage of the germanium resistor bridge. Thts gives the overall transition about a $5 \%$ error, but a much less error is associated for smaller regions. In this way, the film's resistive transitions were measured and recorded both on an analog trace ( $x-y$ recorder) and stored in digital form by a computer ( $A / D^{\prime} s$, and a Nova 1200 ).

The general features of the films studied were: 1) the resistivities were $P_{n} \sim 10^{-5}+10^{-6} \Omega \mathrm{cm}$; and therefore (using the relationship between $e_{e f f}$ and $R_{D}^{N}$ );2) the mean free path was 
$e_{\text {eff }} \sim 10+100 \AA_{;}$3) the f11ms had mostly 1000 A thicknesses (as measured with a quartz thicknass monitor and later checked by optical methods); 4) they had $J_{c}$ 's $\sim 1.54 \mathrm{~K} * 1.93 \mathrm{~K}$, but predominantly in the $1.7 \mathrm{~K}+1.9 \mathrm{~K}$ range; 5) the transition width were broadened for the dirtier f1lms and ware $\Delta T \sim 14+200$ mk, where $\Delta T=T(R N)-T(2 / 3 R N)$; and 6$)$ the ratio of room temperature resistance to the nomal resistance was $\sim .63+1.2$. The heavily oxidized ftlans had their transitions al tered with $D$. C. currents, and ware roore likely to have multiple transitions.

To compare more detailed features of the films, involves fitting the film to an A.L. form for the transition (see Theory Section of thesis) and comparing to otherwork the fitted transition width $\left(\tau_{0}\right)$. A. L. Theory works only for a smalt part of the transition $\left(T>T_{c}\right)$, and thus the comparison of the fitted width paraneter to other workers depands on how auch of the transitian is used th the fit. Strongin, 51 et.al, , have made aluminum firms wi th very swall leff $(.2 \AA)$ and which are quite thin $(100 \AA)$. They observe that thefr fitted $\tau_{0} \sim 10 \tau_{0}^{A . L .}$. Bhatnagar, ${ }^{52}$, et.al., have measurad aluminum film transitions for number of thicknesses. They find the thicker fflms ( $\sim 1000 \AA$ ) have a brosdening of the transition width $\left(\tau_{0} \sim 10 \tau_{0}^{A . L}\right)$, but also a considerably lower $T_{c}(1,3 K)$, Which implies their f1tms are quite clean and would meed a Mak1-Thompson corruction to explain the broadenfing (see Theory section). Kajimurs and Mikoshibe ${ }^{53}$ have done extensive work on dirty aluminum fflms. They find that as one goes to claaner 
systens ( $l_{\text {eff }}$ larger), they get less agreement for the A.L. width parameter $\left(t_{0} \sim 10 \tau_{0}^{A . L}\right.$. for $\left.\varepsilon_{e f f} \sim 1000 \mathrm{~A}\right)$. In their later work (ref. 54), they include the Maki-Thompson correction and fit their data to theory involving both the A.L. term and the Maki-Thompson term. They determine an empirical relatfonship between the pair breaking parameter $(\delta(0))$ and $R^{N}$ where:

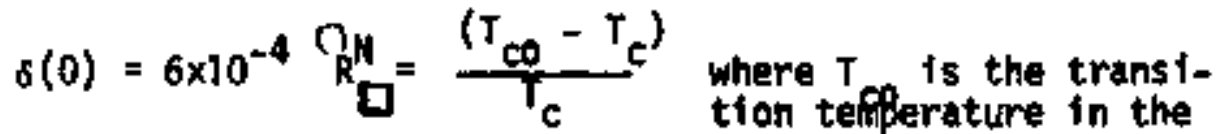

$$
\begin{aligned}
& \text { absence of the pair } \\
& \text { breaking. }
\end{aligned}
$$

This means that the dirtier fi ims, which had a larger $R^{N}$ due to a smaller leff, had a larger pair breaking mechanism. They mention the possibility of the $\mathrm{O}_{2}$ in the $\mathrm{Al}_{2} \mathrm{O}_{3}$ acting as a diamagnetic material involved in the pair breaking. They mainly feet the machanism in pair breaking is due to a localized moment and a proximity effect with the substrate. Thus, one would expect the dirtier fijms not to have a Maki-Thompson correction in the conductivity because of the enhanced palr breaking. Masker and Parks, ${ }^{38}$ also find the dfsagreement with the $\tau_{0}^{A . L}$. to be in the cleaner films. It should be noted that most of this work involves films that have very small width to length ratio $\left(\sim 10^{-3}\right)$ and are in a zig-zag pattern. The total area of their films is on the order of the fllms studied in this experiment $\left(1 \mathrm{~cm}^{2}\right)$. Since the films studied in this experiment tended to be broadened and have transition widths wider than the expected $\tau_{0}^{A} . L$. one might 
suspect the fllms were broadened by inhomogeneitfes. But, due to the large difference in the goometry of the samples studied when compared to the other work mentloned, there might be another explanation for the broadening.

Patton's theory (see the Theory section of this thesis) was used in comparing many experiments on aluminum films by Parks, et.at. 55 shown in Fig. H-6. A value for the pair breaking parameter $(\delta(0))$ of $2 \times 10^{-4}$ was chosen (in the limit of weak pair breaking, where Patton's theory is valid, 1.e., $\delta(0)=$ $\left.t_{\infty} \leq 0.3\right)$. They plot the ratio of the excess conductivity divided by the A.L. excess conductivity $\left(\sigma^{\prime} / \sigma^{\prime A}\right.$, L.) for a reduced temperature of $\varepsilon=0.03$ (chosen because of not as dependent on $R_{\square}^{N}$ for smalle) rs, the normal sheet reststance $R_{\square}^{N}$. The A.L. theory is a straight horizontal line (independnet of $R_{\square}^{N}$ ) and $\sigma / \sigma$ A.L. $=1$. The Thompson Theory is also a straight line with $\sigma^{1} / \sigma^{A} A \cdot \mathrm{L} \approx 11$. Then by plotting many experiments on $\mathrm{Pb}$, $S n, A l$, and $B 1$, they show that the strong pair braking systems (Pb, Bi) are near the A.L. theory, and they have a pair breaking paraneter of $\tau_{c o}=.02$. But the alumínum data shows a curve that agrees with the A.L. theory for large $R_{G}^{N}$ and goes smoothly over to the Thompson theory for small $R_{\sigma}^{N}$ (Targe pair breaking). By using the value of $\tau_{c o}=2 \times 10^{-4}$, Patton's theory is able to agree well with all the data in alumi num films plotted. This Dlot then could be a conventent method of showing where the films studied in this experiment agree with the films studfed by other 


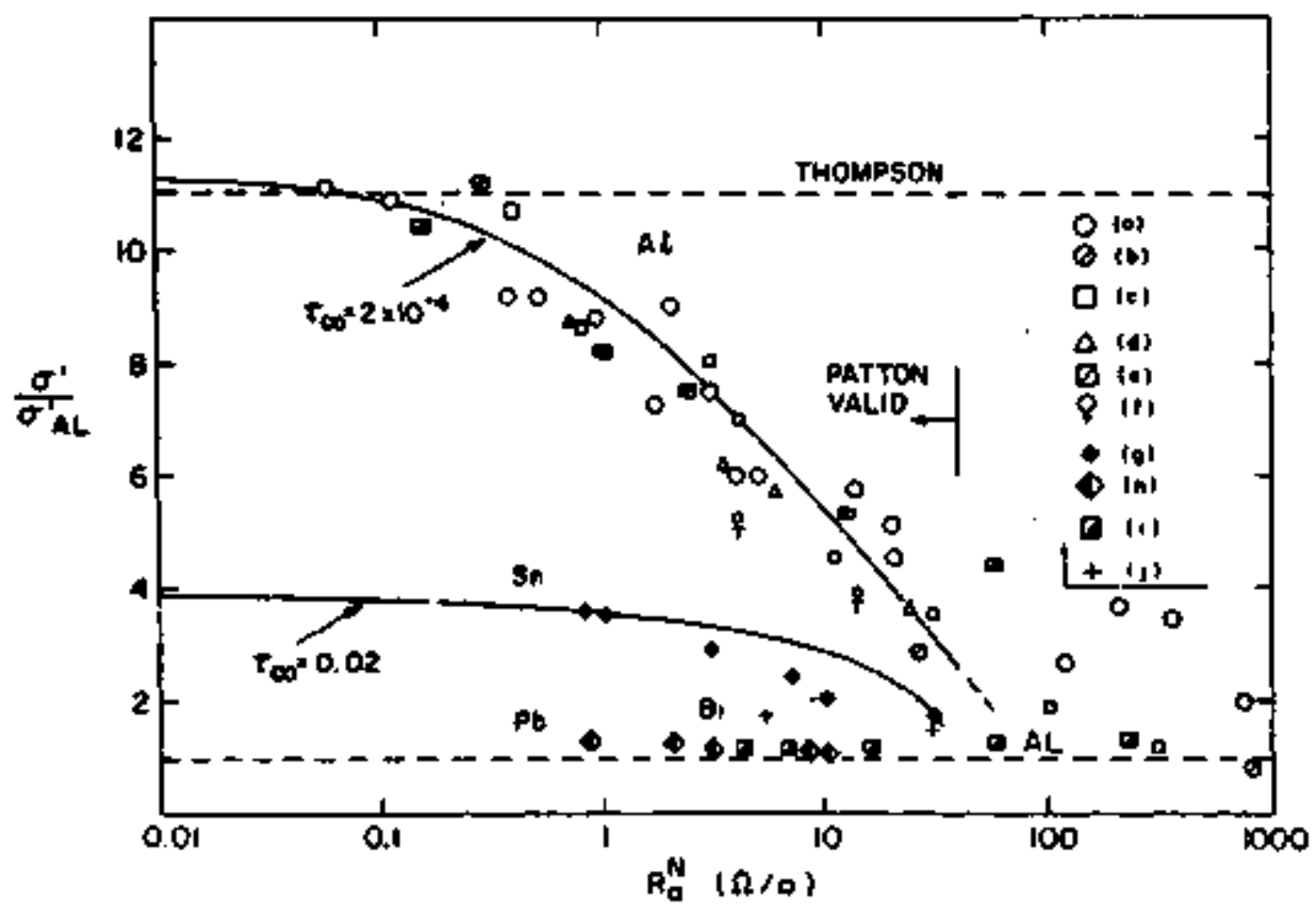

Fic. 5. Ratio of the excets consucturly measured at $₹>0,03$ Get. 47) to that expected trum the AL. thes ry a a func.

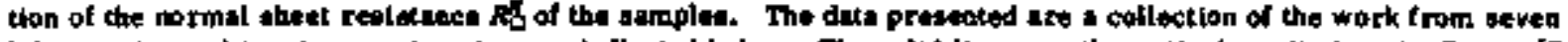

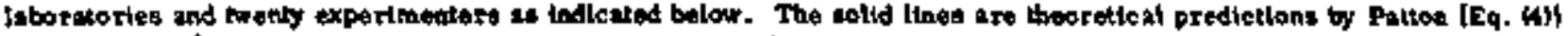

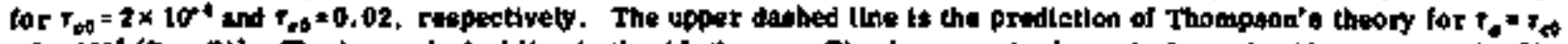

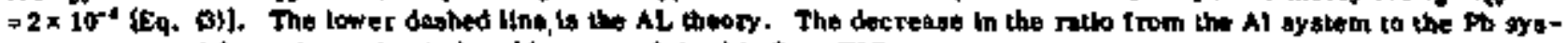
tern is expected trom the rmal padr breaklas at explained in Soc. IV D.

Figure $\mathrm{H}-6$. 
workers, since alt films have the curle-Woiss form $\left(\sigma^{\prime} a \varepsilon^{-1}\right)$, but differ in the width of the transftion. But, the arbitrariness of choosing the plane of the plot only for the value of $E=.03$ distorts the overall understanding, since the ratio of $\sigma^{*} / \sigma^{A}{ }^{A} L$. depends on where in the transition (the value of $\varepsilon$ ) one is looking. Also, $R^{N}$ is not such a unlque parameter, since films of very Targe $R^{N}$ are very thin $(\sim 100 \AA)$ and have quite a different geometry and substrate relationship than films of smal ler $R_{\square}^{N}$ and thus thicker samples. The ratto of $\sigma^{\prime} / \sigma^{\prime A . L}$. can be also written as :

$$
\frac{\sigma^{\prime}}{\sigma^{\prime A . L .}}=\left[\frac{R_{N}-R}{R} \frac{1}{R_{d}^{N}}\right]\left[\frac{1.52 \times 10^{-5} R_{d}^{N}}{R_{g}^{N} d}\right]-1 \text { where } \varepsilon=\frac{T-T_{c}}{T_{c}}
$$

for $\varepsilon=.03$, then $T=1.03 \mathrm{~T}_{\mathrm{c}}$ and eq. $\mathrm{H}-8$ becomes:

$$
\left.\frac{\sigma^{\prime}}{\sigma^{\prime 4 . L .}}\right|_{\varepsilon=.03}=\left[\frac{R N}{R\left(T=1.03 T_{c}\right)}-1\right] \frac{1.97 \times 10^{3} \mathrm{Q} / \mathrm{sq}}{R_{\sigma}^{N}}
$$

Thus if the ratio $R N R\left(T=1.03 T_{c}\right)$ is $\sim 2$ for large $R_{\square}^{N}$ and very close to 1 for $5 m a l 1 R_{m}^{N}$, one can see how the plot in Ftg. H-6 is Just showing the inverse relationship of $\sigma / \sigma^{\text {A.t. }}$ to $R^{N}$. This does not imply that is all it is showing, but just that the features of the data on aluminum are coming from the value for the ratio $\mathrm{PA} / \mathrm{R}\left(\mathrm{T}=1.03 \mathrm{~T}_{\mathrm{C}}\right)$ at different values of $\mathrm{R}_{\mathrm{D}}^{N}$, 
and that thechoice of the rationas arbitrary. It should also be noted that the rejationship shown aarifer between $R_{\square}^{N}$ and $l_{e f f} x d$ (thickness), implies that one cannot tell the difference on the plot of Fig. H-6, between a dirty thick film ( \&eff small, d lerge) and a clean thin film ( $2_{\text {eff }}$ large, $d$ small). In fact sore of the date used in the plot of Fig. H-6 is from ref. 38, which involved work on films that had extremely wide transitions (600 $\mathrm{mK}$ ) and were quite thin ( $150 \AA$ ) . Since one might expect the proximity -ffect involvement in pair breaking (mentioned earlier) to be seen in thinner films, and since short mean free path films also axhibit strong pair breaking, the agreament of the plot in Fig. H-6 for large values of $R_{D}^{N}$ may be related to the inverse of the product of $\ell_{\text {eff }}$ and $d$ rather than $R^{N}$. And, consequently, the thicker films' disagreement with A.L. theory may be due to the absence of pair breaking from larger $\ell_{\text {eff }}$ and less of a substrate proximity effect, rather than simple relationship of just smatl $R_{\square}^{N}$ shown. Thus, the apparent understandfing of resistive transitions shown in Fig. $\mathrm{H}-6$ is misleading. A similar plot for the films used in this experiment indicates reasonable agreement with the A.L. theory in that it is horfzontal for $811 R^{H}$, but at a higher ratio of $\sigma^{\prime} / \sigma^{\prime A}, L$., since the plot involved $\sigma^{\prime} / \sigma^{\prime A} . L$. for a $\sigma^{\prime}=\tau_{0} \varepsilon^{-1} \sigma_{\mathrm{N}}$ fit for all $\varepsilon$. When $\varepsilon$ is large (.03) the agreement can be made qu1te good and films are closer to the ratio of 10 for $R_{a}^{N} \sim 1 \Omega / S Q$ A better approach is to use the graphic plots of $R^{\prime}(=(R N)(R)(R N-R))$ vs. $T$, and fit the data to a straight line in some region. If 
$T_{c}{ }^{* T}(R=0)$, then $T=1.03 T_{c}$ is the region in the $R^{\prime}$ plot where the best value of $\tau_{0}\left(\tau_{0}=\frac{R N}{\tau_{c}\left(\frac{d R^{2}}{d}\right)}\right)$ for the fijms studied is obtained, and the fitms agree with the plot of Fig. H-6.

In conclusion, the films studied have a short $\ell_{e f f}$ and should have a somewhat good agreement with the A.L. theory, which does not involve pair breaking. But, because of the thicknesses of the films studfed, there mfght be a proximity effect pair breaking mechantsm. The films do fit an $\varepsilon^{-1}$ behavior, but with larger transition widths $\left(t_{0}\right)$. The films cannot be directiy comparad to the other workers because of the large differences in the geometry and current densitfes used in the studies. There is the possfbility that the films are inhomogenious ly broadened, since $\tau_{0}$ is larger than $\tau_{0}^{A}$. L. . But if one chooses a sm:11 enough region to f1t to A.L. theory, this disagreement can be partially removed. The agreement with theory was best for films made without $\mathrm{O}_{2}$ on a rigid substrate. 
References for Appendices

1. Cryocal Inc., 1371 Ave. "E", Riviere Beach, Florida, 33404.

2. J. T. Anderson. "Thermal Phase Fluctuations in Josephson Junction...". Ph. O. Thesís, $U$ of Minn., 1971, unpublished.

3. J. J. Hi11, A. P. Mijler, "Seven Decade AdJ-Ratio... Votage Divider...". Proceed. of IEE, 109, part B, 157 (1962);

J. J. Hill, A. P. Miller. "An A.C. Double Bridge...", Proceed. of IEE, 110, 453 (1963).

4. Automatic Systens Laboratories, Construction House, Grovebury Rozd, Leighton Buzzard, Bedfordshire, Engl and.

5. J. C. Solinsky, "Operation of GaRs LED's and Si Detectors at $4.2 \mathrm{~K}^{4}$, Rev, Sc1. Inst., 41, 1886 (1970).

6. Light gutdes were the "Crof on" fiber optics, avaliable from Ediund Scientific Co., Barrington, N. J., in bundle sizes of $16,32,48,64$, fibers per bund le, with a transintss ion range of $13,300 \AA$ to $3,300 \AA$.

7. Light bulbs available from Axialight Corp. (formsally starlight Corp), P. O. Box 325, Mountain Home, North Carolina, 28758. Model 15-2260- 1.5 v, 15 ma. was used extensively. Those bulbs are only $60 \mathrm{mil}$. Iang and $22 \mathrm{mil}$. In djameter, with a filanent of 8s turns on about a $5 \mathrm{mil}$. diameter and only about $40 \mathrm{mil}$. long, yet their output powers are up to $150 \mathrm{w}$ watts.

8. See for a complete review the article by J.E. Zimmerman, Proceed. of IEE 1972 Appl. Superconduct ivity Conf., Annapol is Mary i and, May 1-3, \{1972\} pg. 544. 
9. J. E. Zinuernan, P. Thiene, J. T. Harding, J. App 1. Phys. 4l, '1572 (1970).

10. 5.H.E. Manufacturing Co., 3422 Tripp Court, Suite 8, San Diego, Caltfornia 92121.

11. Richard J. Warburton, "Fluctuations Near the Phase Transition in I-D Superconductors", Ph. D. Thesis, Cornell University, Ithaca, N. Y. (1971).

12. \$. F, Strait, "Development of Mech. Stable Thermally Cyclicable Point Contacts for use in Superconducting Devices", Hs. Thesis, Cornel1 University, Ithaca, N. Y., (1971):

13. O. R. Nordl und, Rev. Sci, Inst., 43, 1699 (1972).

14. J. Keighiey, P. Shodes, Infd Phys., 12, 277 (1972).

15. James, Snith, Wolford, "Analog and Oigital Computer Methods" or any Analog Computer Manual (1.e., Electronics Associates inc., "Handbook of Analog Computation").

16. J. H. T. Dabbs, "Wethod for Obtaining Second Derivatives in Electron Tunneling", preprint. Also see, D. E. Thomas, J. M. Rowell, Rev. Sci. Inst., 35, 1301 (1965) and W. R. Patterson, J. Schewchun, Rev. Sct. Inst, 35, 1704 (1964).

17. J. E. Houston, R. L. Park, Rev. Sct, Inst, 43, 1437 (1972).

18. R. B. BTackman, J, W. Tukey, "The Measurement of Power Spectra", Dover Publications (1958).

19. S. Brandt, Statistical and Computational Methods in Data Analysts , Morth Holland Publ. Co. (1970). 
20. P. Sullivan, 6. Seidel, Proceed, of 1966 LT-Cal. Conf., Ann. Acad. Scf. Fennicae., A VI, \#210, pg. 58 (1966);

P. Sullivan, G. Seitiel, Phys, Rev. $\underline{173}, 679$ (1968);

P. Handler, D. Mapother, M. Rayl, Phys. Rev. Letters 19,

356 (1967). It should be noted that N. Zavaritski $i$ (Progress

in Cryogenics, 1, 207, Heywood \& Co., London (1959)) first

developed A.C. methods, but it was in a more complicated nature of phase shifts with thermal waves, rather than simply steady state amplitude variations.

21. The imaginary part of the index of refraction for $k$ for this wave length is about 6.00 (see Heavens. Optical Properties of Thin Solid Eilms, Dover 1965) Then $\alpha=2 \pi / \lambda 6 \times 10^{5} \mathrm{~cm}^{-1}$.

22. 5. Cranda11, Quar. of App1, Math, 13, 318 (1955).

23. R. Zerkle, J.Saunderland, J. Heat Transfer, 87, 117 (1965).

24. G. K. In1te, Exp. Tech. in Low Temp. Physies, Oxford, England (1968).

25. R. C. Weast, C, R, C. Handbook of Chem. \& Physics, The Chemt cal Rubber Co., Ohio (1971).

26. C. B, Satterthwaite, Phys, Rev. 125, 873 (1962).

27. R. L. Greene, C. N. King, R, B. Zubeck, J. J. Hauser, Phys, Rev. B6, 3297 (1972).

28. D. E. Gray, AIP Handbook (page 4-T59), McGraw Hill, K.Y. (1971).

29. K. K. Kelley, J. Ar. Chem, Soc, 61, 471 (1939).

30. P. Mahadev, M. F. Panczyk, R. A. Scribner, J, G. Daunt, Phys, Letters 41A, 221 (1972). 
31. W. E. Keller, $\mathrm{He}^{3}+\mathrm{He}^{4}$, Plenum Press, New York 1969.

32. D. H. Martin, O. Bloor, Cryogenics 1. 159 (1961).

33. C. L. Aertin, R. Rose, J. Appl. Phys. 39, 2561 (1968),

34. L. R. Testardi, Phys. Rey. 84, 2189 (1971).

35. W. H. Parker, W. D. Will iams, Phys. Rev. Letters 29, 924 (1972).

36. C. S. Owen, D. J. Scalipino, Phys. Rev, Letters 28, 1559 (1972).

37. D. H. Liebenberg, Los Alamos, N. M., Private communication.

38. W. Masker, R. Parks, Phys, Rev, Bl, 2164 (1970).

39. H. Oona, D. 6. Rickel, Rev. Sct. Inst. 38, 980 (1967);

A. Imbusch, Rev. Scí. Inst. $\underline{38}, 974$ (1967):

G. Munch, Rev. Sci. Inst. 35,524 (1964).

40. G. J. Unterkofier, and R. Verderber, Rev. Sci. Inst. 34, 820 (1963).

41. F. Hooten, Rev. Sci. Inst. 38. 124 (1967).

42. G. Deutscher, H. Fentche1, M. Gerschenson, E. Grunbaum, International Low Temperature Conference $\# 13$, Boulder, Colorado, Paper \# 6s59 (1972).

43. H. A. Pankratov, G. A. Zaitsev, I. A. Khrebtov, Cryogenics, Apri1, 242 (1973).

44. L. R. Testardi, Phystes Letters 35A, 33 (1971).

45. 6. Deutscher, Physics Letters 35A, 28 (1971);

P. Pellan, G. Dousselin, H. Cortès, J. Rosenblatt, Solid State Comm. 11. 427 (1972).

46. M. Strongin, 0. F. Karmerer. A. Paskin, Phys. Rev. Letters 14, 949 (1965); P. H. Chubov, V. V. Erenenko, Yu. A. P1Itpenko, Sovtet Phys., JETP 28, 389 (1969). 
47. A. Rothwarf, Physics Letters 30A, 55 (1969).

48. J. R. Tucker, B, 1, Hatperin, Phys, REv, B3, 3768 (1971).

49. D. Saint James, G. Sarma, Type II: Superconductfylty, Pergamon Press, New York (1969) pg. 46.

50. B. Abeles, R. W. Cohen, W. R. Stowe11, Phys, Rev, Letters 18, 902 (1967); R. W. Cohen, B. Abetes, Phys. Rev. 168, 444 (1968).

51. M. Strongin, O. F. Kanmerer, J. Crow, R. S. Thomps on, H. L. Fine, Phys. Rev, Letters 20, 922 (1968). -

52. A. K. Bhatnagar, P. Kahn, T, J. Zannft, Solid State Comm, 8 , 79 (1970).

53. K. Kajlmura, N. Mikoshiba, Physics Letters 32A, 216 (1970).

54. K. Kajimura, N. Mjkoshiba, J. of Low Temp. Phys. 4, 331 (1971).

55. R. A. Craven, G. A. Thomas, R. D. Parks, Phys. Rev, 87, 157 (1973).

56. The heat capact ty of the mica used for the substates was measured Independently using conventionat methods by Prakash Thadant and extrapolates to $C=1.1 \times 10^{-4} \mathrm{~J} / 9 \mathrm{~g}^{\circ} \mathrm{K}$ at $1.83 \mathrm{~K}$ with no unusuz 1 behavior in this region. The author is deeply grateful to Prakash Thadani for obtaining these results. 


\section{Acknowledgements}

The thesis advisor and friend of the author, Allen $M$. coldonan, suggested this work. It was through the constant cormunication and mutual support that this work was completed. Without this open and frank relationship that endured between the author and Professor 6oldman and a lot of luck, this work would not have been possible.

The author would like to thank Professor Allen M. Goldman for the counsel and aid recelved during this work and for the encouragement and confidence in the author's research.

The author acknowledges the support for this research by the Metal turgical Division of the A.E.C. on Contract WAT(11-1)-1569. The author also thanks the Office of Haval Research for providing the He gas used in this experiment. A grant from the Research Corporation made possible the mini-computer used in this work. The Graduate School of the University of Minnesota made funds avallable for the Tektronix display systent. The author also acknowledges Earl Klugman for the extended loan of the P.A.R. th 26 lock-in used in this work.

The author thanks T. Hutchinson and K. Muns on for the assistance with the TEM meas urements. The author is grateful to Prakash Thadani for the heat capacity measurements made on the mica substrates. The skills of Don Gooding in the cryostat construction and SQUIO fabrication made some experinental aspects of this work 
possible. The evaporator system used for ft $1 \mathrm{~m}$ preparation was bullt by Peter Kreisman. It was through the urging of Patrick Lee that the magnetic field studies were made. The interaction with fellow graduate students was constant source of entightenment. Finally, without the love and understanding of the author's graduate-widow wife and famtly and their desire to return to a more liveable elfmate, this work would not have been possible. 